\title{
Catalytic Ring-Opening of Cyclic Alcohols Enabled by PCET Activation of Strong O-H Bonds
}

Hatice G. Yayla, Huaiju Wang, Kyle T. Tarantino, Hudson S. Orbe, and Robert R. Knowles*

Department of Chemistry, Princeton University, Princeton, New Jersey 08544, United States

Correspondence to: rknowles@princeton.edu

Supporting Information

Table of Contents $\quad$ Page

General Information $\quad$ S2

Synthesis of Starting Materials $\quad$ S3

$\begin{array}{ll}\text { Synthesis of Products } & \text { S14 }\end{array}$

${ }^{1} \mathrm{H}$ and ${ }^{13} \mathrm{C}$ NMR Spectra of Products $\quad$ S28

Stern-Volmer Studies $\quad$ S63

Experimental Details for Investigating Long-range PCET (Figure 3) S66

Experimental Details for Investigating the Relationship $\quad$ S68

Between Effective BDFE and Reaction Outcomes (Figure 4)

$\begin{array}{lr}\text { Cyclic Voltammograms of Starting Materials } & \text { S69 }\end{array}$

Structure and Potential Data of Photocatalysts $\quad$ S76

$\begin{array}{ll}\text { DFT Computations } & \text { S78 }\end{array}$

$\begin{array}{ll}\text { References } & \text { S91 }\end{array}$ 


\section{General Information}

Commercial reagents were purified prior to use following the guidelines of Perrin and Armarego. ${ }^{1}$ All solvents were purified according to the method of Grubbs. ${ }^{2}$ Organic solutions were concentrated under reduced pressure on a Büchi rotary evaporator using a water bath. Chromatographic purification of products was accomplished by flash chromatography on a Biotage Isolera One with cartridges containing Fluka 230-400 mesh silica gel or Acros Brockmann I 70-290 mesh neutral alumina. Thin-layer chromatography (TLC) was performed on Silicycle $250 \mu \mathrm{m}$ silica gel plates or Sorbent Technologies $250 \mu \mathrm{m}$ neutral alumina plates. Visualization of the developed chromatogram was performed by irradiation with UV light or treatment with a solution of potassium permanganate, ceric ammonium molybdate, or 2,4dinitrophenylhydrazine stain followed by heating. Yields refer to purified compounds unless otherwise noted.

All ${ }^{1} \mathrm{H}$ and ${ }^{13} \mathrm{C}$ NMR spectra were recorded on Bruker Avance II $500(500$ and $126 \mathrm{MHz}$ for ${ }^{1} \mathrm{H}$ and ${ }^{13} \mathrm{C}$ respectively), Bruker Avance III $300\left(75 \mathrm{MHz}\right.$ for $\left.{ }^{13} \mathrm{C}\right)$, or Bruker Avance III HD $800\left(800 \mathrm{MHz}\right.$ for $\left.{ }^{1} \mathrm{H}\right)$ instruments, and are internally referenced to residual protio-solvent signals: $\mathrm{CDCl}_{3}$ at $\delta 7.26$ and $77.16 \mathrm{ppm}$ and $\mathrm{C}_{6} \mathrm{D}_{6}$ at $\delta 7.16$ and $128.06 \mathrm{ppm}$. Data for ${ }^{1} \mathrm{H}$ NMR are reported as follows: chemical shift $(\delta \mathrm{ppm})$, broad peak (b), multiplicity ( $\mathrm{s}=$ singlet, $\mathrm{d}=$ doublet, $\mathrm{t}=$ triplet, $\mathrm{q}=$ quartet, $\mathrm{p}=$ pentet, $\mathrm{h}=$ hextet, $\mathrm{m}=$ multiplet $)$, coupling constant $(\mathrm{Hz})$ and integration; data for ${ }^{13} \mathrm{C}$ NMR are reported in terms of chemical shift and no special nomenclature is used for equivalent carbons. IR spectra were recorded on a Perkin Elmer Spectrum 100 FT-IR spectrometer and are reported in terms of frequency of absorption $\left(\mathrm{cm}^{-1}\right)$. High-resolution mass spectra were obtained at Princeton University Mass Spectrometry Facility using an Agilent 6210 TOF LC/MS (Electrospray Ionization) or an Agilent 7200 Q-TOF GC/MS (Electron Ionization); low-resolution ones were obtained on an Agilent $6120 \mathrm{LC} / \mathrm{MS}$ (Electrospray Ionization) or an Agilent 5975C GC/MSD (Electron Ionization). Cyclic voltammograms were acquired on a $\mathrm{CH}$ Instruments $600 \mathrm{E}$ potentiostat. Stern-Volmer experiments and measurement of the emission spectrum of photocatalyst were conducted on an Agilent Cary Eclipse Fluorescence Spectrophotometer. 


\section{Synthesis of Starting Materials}

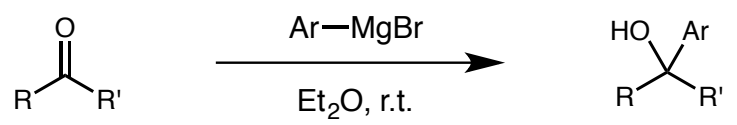

\section{Procedure A (Grignard solution):}

A flame-dried $250 \mathrm{~mL}$ round bottom flask was charged with a stir bar and degassed. Commercial 4-methoxyphenylmagnesium bromide solution (2.0 equiv, $0.5 \mathrm{M}$ THF solution) was syringed into the flask. The relevant ketone (1.0 equiv) was added dropwise into the solution. The reaction was stirred at room temperature and consumption of starting material was monitored by TLC. Upon completion, the reaction mixture was quenched by slow addition of ice water. The aqueous layer was extracted with ethyl acetate. The combined organic layers were washed with brine, dried over anhydrous sodium sulfate, concentrated and purified by neutral alumina flash column chromatography (hexanes/EtOAc) to obtain the pure alcohol.

\section{Procedure B (Grignard):}

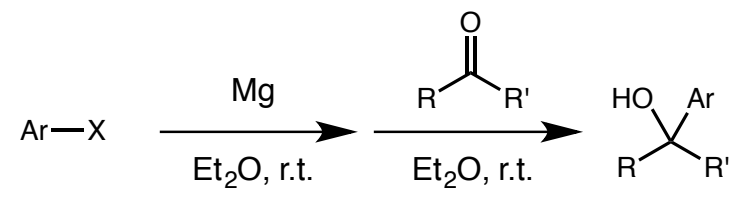

A flame-dried $250 \mathrm{~mL}$ round bottom flask was charged with a stir bar, catalytical amount of iodine, 1-iodo-4-methoxybenzene (1.5 equiv), and magnesium (1.75 equiv). The flask was then evacuated and carefully refilled with argon. $\mathrm{Et}_{2} \mathrm{O}$ was added to make a $0.5 \mathrm{M}$ solution of the aryl iodide, and the mixture was stirred at room temperature under Ar for 45 minutes to allow complete formation of the aryl Grignard reagent. The solution was cooled to $0{ }^{\circ} \mathrm{C}$ in an ice bath, and the ketone (1.0 equiv, 1.0 $\mathrm{M} \mathrm{Et}_{2} \mathrm{O}$ or THF solution) was added in dropwise. The reaction was allowed to warm to room temperature and consumption of ketone was monitored by TLC. Upon completion, dropwise addition of $\mathrm{H}_{2} \mathrm{O}$ quenched the reaction. The aqueous layer was extracted three times with EtOAc. The combined organic layers were washed with brine, dried over anhydrous $\mathrm{Na}_{2} \mathrm{SO}_{4}$, concentrated, and purified by neutral alumina flash column chromatography (hexanes/EtOAc) to afford the pure alcohol.

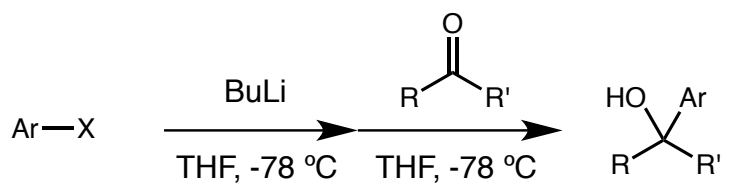

\section{Procedure C (Lithiation):}

Aryl bromide or the parental arene (1.0 equiv) was added into a flame-dried $100 \mathrm{~mL}$ round bottom flask containing a stir bar, and dissolved in anhydrous THF. The solution was purged with argon and cooled to $-78{ }^{\circ} \mathrm{C}$. Then $n$-BuLi (1.1 equiv) or $t$-BuLi (2.0 equiv) was added slowly into the solution. The reaction mixture was stirred at $-78{ }^{\circ} \mathrm{C}$ and lithiation monitored by GC. When the aryl bromide was fully consumed, the ketone (1.0 equiv) was added dropwise via syringe. The reaction was allowed to warm up to room temperature and the consumption of ketone was monitored by TLC. Upon completion, the reaction mixture was quenched by slow addition of $30 \mathrm{~mL}$ saturated ammonium chloride. Organics were extracted three times with EtOAc. The combined organic layers were washed with brine, dried over anhydrous $\mathrm{Na}_{2} \mathrm{SO}_{4}$, concentrated, and purified by neutral alumina flash column chromatography (hexanes/EtOAc) to obtain the pure alcohol. 


\section{1-(4-methoxyphenyl)cyclohexan-1-ol}

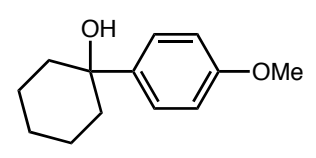

The titled compound was prepared according to a literature procedure. ${ }^{3}$ Spectra are consistent with reported literature values. $\boldsymbol{E}_{\mathbf{p} / \mathbf{2}}\left(\mathbf{v s} . \mathbf{F c} / \mathbf{F c}^{+}\right)=1.22 \mathrm{~V}$.

\section{1-(4-methoxyphenyl)cyclobutan-1-ol}

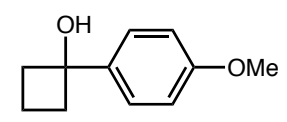

Prepared following the general procedure A with cyclobutanone to afford the titled compound as a colorless oil $(2.1 \mathrm{~g}, 88 \%)$. ${ }^{1} \mathbf{H}$ NMR $\left(\mathbf{5 0 0} \mathbf{M H z}, \mathbf{C}_{\mathbf{6}} \mathbf{D}_{\mathbf{6}}\right) \delta 7.32(\mathrm{~d}, J=8.7 \mathrm{~Hz}, 2 \mathrm{H}), 6.81(\mathrm{~d}, J=$ $8.7 \mathrm{~Hz}, 2 \mathrm{H}), 3.33$ (s, 3H), 2.37 (dddd, $J=11.6,9.2,5.3,2.7 \mathrm{~Hz}, 2 \mathrm{H}), 2.25-2.14$ (m, 2H), 1.83 (tdd, $J=9.4,5.9,4.5 \mathrm{~Hz}, 1 \mathrm{H}), 1.51$ (dddd, $J=16.4,11.1,8.8,7.6 \mathrm{~Hz}, 1 \mathrm{H}) .{ }^{13} \mathbf{C}$ NMR (126 MHz, $\left.\mathbf{C}_{6} \mathbf{D}_{6}\right) \delta 158.9,139.0,126.2,113.6,76.2,54.5,37.2,13.0$. IR (neat): 3392, 2984, 2940, 2836, $1611,1512,1299,1177,1030,828 \mathrm{~cm}^{-1}$. HRMS (EI): exact mass calculated for $[\mathrm{M} \bullet]^{+}$ $\left(\mathrm{C}_{11} \mathrm{H}_{14} \mathrm{O}_{2}\right)$ requires $\mathrm{m} / \mathrm{z} 178.0988$, found $\mathrm{m} / \mathrm{z} 178.0992$.

\section{1-(4-methoxyphenyl)cyclopentan-1-ol}

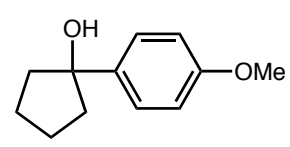

Prepared following the general procedure A with cyclopentanone to afford the titled compound as a colorless oil $(2.8 \mathrm{~g}, 64 \%) .{ }^{1} \mathbf{H} \mathbf{N M R}\left(\mathbf{5 0 0} \mathbf{M H z}, \mathbf{C}_{\mathbf{6}} \mathbf{D}_{\mathbf{6}}\right) \delta 7.32(\mathrm{~d}, J=8.8 \mathrm{~Hz}, 2 \mathrm{H}), 6.82(\mathrm{~d}, J$ $=8.8 \mathrm{~Hz}, 2 \mathrm{H}), 3.35(\mathrm{~d}, J=2.1 \mathrm{~Hz}, 3 \mathrm{H}), 1.99-1.84(\mathrm{~m}, 2 \mathrm{H}), 1.79(\mathrm{ddd}, J=11.5,5.4,2.4 \mathrm{~Hz}$, 4H), 1.62 (ddd, $J=10.6,5.2,1.9 \mathrm{~Hz}, 2 \mathrm{H}) .{ }^{13} \mathbf{C}$ NMR (126 MHz, $\left.\mathbf{C}_{\mathbf{6}} \mathbf{D}_{\mathbf{6}}\right) \delta 158.6,139.6,126.3$, 113.4, 82.5, 54.5, 41.7, 23.8. IR (neat): 3405, 2955, 2872, 2836, 1611, 1581, 1511, 1463, 1442, $1365,1297,1240,1177,1114,1093,1033,1000,961,904,883,795,732 \mathrm{~cm}^{-1}$. HRMS (ESI): exact mass calculated for $\left[\mathrm{M}-\mathrm{H}_{2} \mathrm{O}+\mathrm{H}\right]^{+}\left(\mathrm{C}_{12} \mathrm{H}_{15} \mathrm{O}\right)$ requires $m / z$ 175.11175, found $m / z$ 175.11164.

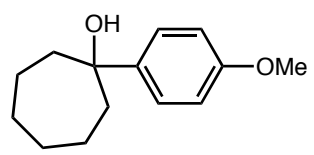

\section{1-(4-methoxyphenyl)cycloheptan-1-ol}

Prepared following the general procedure A with cycloheptan-1-one to afford the titled compound as a colorless oil (1.38 g, 74\%). ${ }^{1} \mathbf{H}$ NMR $\left(500 ~ \mathbf{~ H H z}, \mathbf{C}_{6} \mathbf{D}_{6}\right) \delta 7.37(\mathrm{~d}, J=8.8 \mathrm{~Hz}$, $1 \mathrm{H}), 6.84(\mathrm{~d}, J=8.8 \mathrm{~Hz}, 1 \mathrm{H}), 3.36(\mathrm{~s}, 1 \mathrm{H}), 1.88(\mathrm{ddd}, J=14.4,10.6,1.8 \mathrm{~Hz}, 1 \mathrm{H}), 1.82-1.68$ (m, 2H), $1.63-1.54(\mathrm{~m}, 1 \mathrm{H}), 1.52-1.36(\mathrm{~m}, 2 \mathrm{H}) .{ }^{13} \mathbf{C} \mathbf{N M R}\left(\mathbf{1 2 6} \mathbf{M H z}, \mathbf{C}_{\mathbf{6}} \mathbf{D}_{\mathbf{6}}\right) \delta 158.4,143.5$, $125.7,113.4,75.8,54.5,43.3,28.9,22.4$. IR (neat): 3421, 2922, 2856, 1609, 1510, 1460, 1245, $1178,1033,799 \mathrm{~cm}^{-1}$. HRMS (EI): exact mass calculated for [M•] ${ }^{+}\left(\mathrm{C}_{14} \mathrm{H}_{20} \mathrm{O}_{2}\right)$ requires $\mathrm{m} / z$ 220.1458 , found $m / z 220.1459$.

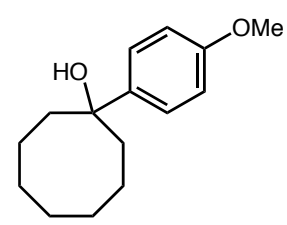




\section{1-(4-methoxyphenyl)cyclooctan-1-ol}

Prepared following the general procedure A with cyclooctan-1-one to afford the titled compound as a colorless oil $(2.97 \mathrm{~g}, 76 \%)$. ${ }^{1} \mathbf{H}$ NMR $\left(\mathbf{5 0 0} \mathbf{M H z}, \mathbf{C}_{6} \mathbf{D}_{6}\right) \delta 7.39(\mathrm{~d}, J=8.9 \mathrm{~Hz}, 2 \mathrm{H}), 6.84(\mathrm{~d}, J$ $=8.9 \mathrm{~Hz}, 2 \mathrm{H}), 3.36(\mathrm{~s}, 2 \mathrm{H}), 1.92(\mathrm{ddd}, J=14.9,8.5,1.8 \mathrm{~Hz}, 2 \mathrm{H}), 1.83(\mathrm{ddd}, J=14.8,9.8,2.2$ $\mathrm{Hz}, 2 \mathrm{H}), 1.76-1.64(\mathrm{~m}, 2 \mathrm{H}), 1.63-1.50$ (m, 3H), $1.48-1.35$ (m, 5H). ${ }^{13}$ C NMR (126 MHz, $\left.\mathbf{C}_{6} \mathbf{D}_{6}\right) \delta 158.5,141.8,126.3,113.3,75.6,54.5,37.9,28.4,24.4,21.9$. IR (neat): 3414,2919 , 2851, 1609, 1510, 1301, 1243, 1178, 1038, 830, $804 \mathrm{~cm}^{-1}$. HRMS (EI): exact mass calculated for $[\mathrm{M} \bullet]^{+}\left(\mathrm{C}_{15} \mathrm{H}_{22} \mathrm{O}_{2}\right)$ requires $m / z 234.1614$, found $m / z 234.1612$.

\section{1-(4-methoxyphenyl)cyclodecan-1-ol}

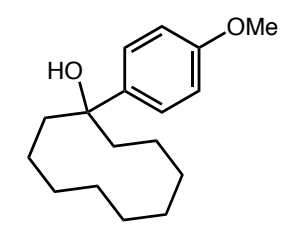

Prepared following the general procedure A with cyclodecan-1-one to afford the titled compound as a white solid (1.86 g, 39\%). ${ }^{1} \mathbf{H}$ NMR $\left(\mathbf{5 0 0} \mathbf{M H z}, \mathbf{C}_{\mathbf{6}} \mathbf{D}_{\mathbf{6}}\right) \delta 7.41(\mathrm{~d}, J=8.9 \mathrm{~Hz}, 2 \mathrm{H}), 6.86(\mathrm{~d}, J$ $=8.9 \mathrm{~Hz}, 2 \mathrm{H}), 3.37(\mathrm{~s}, 3 \mathrm{H}), 1.95(\mathrm{dt}, J=14.4,6.1 \mathrm{~Hz}, 2 \mathrm{H}), 1.83(\mathrm{dt}, J=14.2,6.7 \mathrm{~Hz}, 2 \mathrm{H}), 1.60-$ $1.36(\mathrm{~m}, 14 \mathrm{H}) .{ }^{13} \mathbf{C}$ NMR (126 MHz, $\left.\mathbf{C}_{6} \mathbf{D}_{6}\right) \delta 158.5,141.4,126.3,113.2,76.5,54.4,35.5,26.6$, 26.0, 23.7, 21.6. IR (neat): 3363, 2920, 2845, 1610, 1582, 1510, 1483, 1442, 1297, 1245, 1178, $1038,830 \mathrm{~cm}^{-1}$. MS (EI): exact mass calculated for $\left[\mathrm{M}-\mathrm{H}_{2} \mathrm{O}\right]^{+}\left(\mathrm{C}_{17} \mathrm{H}_{24} \mathrm{O}\right)$ requires $\mathrm{m} / z$ 244.2, found $\mathrm{m} / \mathrm{z} 244.2$.

\section{1-(4-methoxyphenyl)cyclododecan-1-ol}

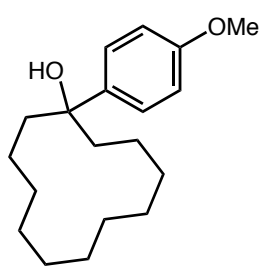

Prepared following the general procedure A with cyclododecanone to afford the titled compound as a white solid (2.93 g, 77\%). ${ }^{1} \mathbf{H}$ NMR (500 MHz, $\left.\mathbf{C}_{\mathbf{6}} \mathbf{D}_{\mathbf{6}}\right) \delta 7.38(\mathrm{~d}, J=8.9 \mathrm{~Hz}, 2 \mathrm{H}), 6.86(\mathrm{~d}, J$ $=8.8 \mathrm{~Hz}, 2 \mathrm{H}), 3.38(\mathrm{~s}, 3 \mathrm{H}), 1.75(\mathrm{ddt}, J=25.0,13.4,7.0 \mathrm{~Hz}, 4 \mathrm{H}), 1.30(\mathrm{tt}, J=18.8,9.8 \mathrm{~Hz}$, 18H). ${ }^{13} \mathbf{C}$ NMR (126 MHz, $\left.\mathbf{C}_{6} \mathbf{D}_{6}\right) \delta 158.5,141.1,126.5,113.2,75.4,54.5,35.6,26.4,26.2$, 22.5, 22.2, 20.0. IR (neat): 3334, 2933, 2848, 1609, 1509, 1470, 1178, 1038, 826, $737 \mathrm{~cm}^{-1}$. MS (EI): exact mass calculated for $\left[\mathrm{M}-\mathrm{H}_{2} \mathrm{O}\right]^{+}\left(\mathrm{C}_{19} \mathrm{H}_{28} \mathrm{O}\right)$ requires $m / z$ 272.2, found $m / z 272.1$.

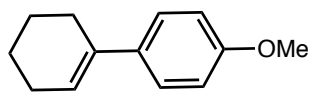

\section{4'-methoxy-2,3,4,5-tetrahydro-1,1'-biphenyl}

The titled compound was prepared according to a literature procedure. ${ }^{3}$ Spectra are consistent with reported literature values.

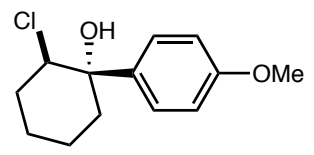

0-2-chloro-1-(4-methoxyphenyl)cyclohexan-1-ol

Trichloroisocyanuric acid $(0.460 \mathrm{~g}, 1.8 \mathrm{mmol})$ was added in small portions to a stirred solution 
of 4'-methoxy-2,3,4,5-tetrahydro-1,1'-biphenyl $(1.000 \mathrm{~g}, 5.3 \mathrm{mmol})$ in a degassed mixture of acetone $(12 \mathrm{~mL})$ and water $(3 \mathrm{~mL})$. Upon completion, aqueous sodium bicarbonate $(30 \mathrm{~mL}$, saturated) and $\mathrm{CH}_{2} \mathrm{Cl}_{2}(30 \mathrm{~mL})$ was added to the solution. The mixture was extracted with $\mathrm{CH}_{2} \mathrm{Cl}_{2}(2 \times 30 \mathrm{~mL})$ and the combined organic layers were washed with brine, drived over $\mathrm{Na}_{2} \mathrm{SO}_{4}$ and concentrated. The crude mixture was purified on silica gel to afford the titled compound as a pale lilac solid as a single diastereomer $(0.95 \mathrm{~g}, 74 \%){ }^{1} \mathbf{H}$ NMR (500 MHz, $\left.\mathbf{C}_{6} \mathbf{D}_{6}\right) \delta 7.30(\mathrm{~d}, J=8.8 \mathrm{~Hz}, 2 \mathrm{H}), 6.80(\mathrm{~d}, J=8.8 \mathrm{~Hz}, 2 \mathrm{H}), 4.14(\mathrm{q}, J=2.8 \mathrm{~Hz}, 1 \mathrm{H}), 3.32(\mathrm{~s}, 3 \mathrm{H})$, $2.40(\mathrm{td}, J=13.1,4.0 \mathrm{~Hz}, 1 \mathrm{H}), 2.30(\mathrm{ddt}, J=14.8,13.4,4.3 \mathrm{~Hz}, 1 \mathrm{H}), 1.86-1.71(\mathrm{~m}, 2 \mathrm{H}), 1.65$ (qt, $J=12.6,3.6 \mathrm{~Hz}, 1 \mathrm{H}), 1.49-1.29(\mathrm{~m}, 3 \mathrm{H}) .{ }^{13} \mathbf{C}$ NMR (126 MHz, $\left.\mathbf{C}_{6} \mathbf{D}_{6}\right) \delta 159.1,138.5$, 127.0, 113.3, 73.7, 65.4, 54.4, 30.6, 30.2, 20.7, 19.2. IR (neat): 3451, 2938, 2864, 1610, 1514, 1443, 1295, 1249, 1180, 1148, 1035, 973, 827, 812, 795, $719 \mathrm{~cm}^{-1}$. MS (EI): exact mass calculated for $[\mathrm{M}-\mathrm{HCl}]^{+}\left(\mathrm{C}_{13} \mathrm{H}_{16} \mathrm{O}_{2}\right)$ requires $m / z$ 204.1, found $m / z$ 204.1.

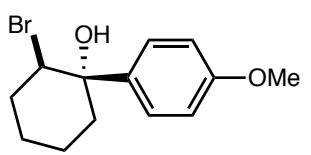

\section{2-bromo-1-(4-methoxyphenyl)cyclohexan-1-ol}

4'-methoxy-2,3,4,5-tetrahydro-1,1'-biphenyl (1.000 g, $5.3 \mathrm{mmol})$ was dissolved in degassed acetone $(10 \mathrm{~mL})$ and water $(5 \mathrm{~mL})$ with $N$-bromosuccinimide $(0.947 \mathrm{~g}, 5.3 \mathrm{mmol})$. The mixture was stirred at $0{ }^{\circ} \mathrm{C}$ for an hour, then allowed to reach room temperature. Upon completion, 30 $\mathrm{mL}$ water was added to the reaction mixture and was extracted with ethyl acetate $(4 \times 30 \mathrm{~mL})$. The combined organic layers were washed with brine, dried over $\mathrm{Na}_{2} \mathrm{SO}_{4}$, and concentrated. The crude mixture was purified on silica gel to afford the titled compound as an off-white solid as a single diastereomer (0.8 g, 53\%). ${ }^{1} \mathbf{H}$ NMR (500 MHz, $\left.\mathbf{C}_{\mathbf{6}} \mathbf{D}_{\mathbf{6}}\right) \delta 7.27(\mathrm{~d}, J=8.9 \mathrm{~Hz}, 1 \mathrm{H}), 6.79$ (d, $J=8.8 \mathrm{~Hz}, 1 \mathrm{H}), 4.31(\mathrm{td}, J=3.7,3.3,2.0 \mathrm{~Hz}, 1 \mathrm{H}), 3.31(\mathrm{~s}, 2 \mathrm{H}), 2.53-2.44(\mathrm{~m}, 1 \mathrm{H}), 2.35$ (ddt, $J$ $=14.2,12.3,3.5 \mathrm{~Hz}, 1 \mathrm{H}), 1.95-1.87(\mathrm{~m}, 1 \mathrm{H}), 1.81$ (qt, $J=12.7,3.7 \mathrm{~Hz}, 1 \mathrm{H}), 1.72-1.60(\mathrm{~m}$, $1 \mathrm{H}), 1.45(\mathrm{dqd}, J=12.7,4.4,3.7,2.1 \mathrm{~Hz}, 1 \mathrm{H}), 1.35(\mathrm{dt}, J=13.4,3.9 \mathrm{~Hz}, 1 \mathrm{H}) .{ }^{13} \mathbf{C}$ NMR (126 MHz, $\left.\mathbf{C}_{6} \mathbf{D}_{6}\right) \delta 159.1,139.0,126.8,113.3,73.5,60.6,54.4,33.5,31.0,20.8,20.2$. IR (neat): 3451, 2937, 2862, 2837, 1612, 1513, 1444, 1293, 1180, 1036, 968, 826, 809, 700, $673 \mathrm{~cm}^{-1}$. MS (EI): exact mass calculated for $\left[\mathrm{M}-\mathrm{HBr}-\mathrm{H}_{2} \mathrm{O}\right]^{+}\left(\mathrm{C}_{13} \mathrm{H}_{14} \mathrm{O}\right)$ requires $\mathrm{m} / \mathrm{z}$ 186.1, found $\mathrm{m} / \mathrm{z}$ 186.2.

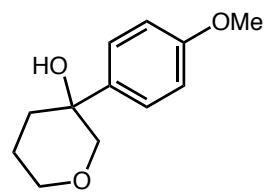

\section{3-(4-methoxyphenyl)tetrahydro-2H-pyran-3-ol}

Prepared following the general procedure $\mathrm{B}$ with dihydro- $2 \mathrm{H}$-pyran-3(4H)-one to afford the titled compound as a white solid (3.03 g, 58\%). ${ }^{1} \mathbf{H}$ NMR (500 MHz, $\left.\mathbf{C}_{6} \mathbf{D}_{6}\right) \delta 7.44(\mathrm{~d}, J=8.9$ $\mathrm{Hz}, 2 \mathrm{H}), 6.83(\mathrm{~d}, J=8.9 \mathrm{~Hz}, 2 \mathrm{H}), 3.73-3.65(\mathrm{~m}, 1 \mathrm{H}), 3.62(\mathrm{dd}, J=11.4,2.3 \mathrm{~Hz}, 1 \mathrm{H}), 3.40(\mathrm{~d}, J$ $=11.5 \mathrm{~Hz}, 1 \mathrm{H}), 3.32(\mathrm{~s}, 3 \mathrm{H}), 3.11(\mathrm{td}, J=11.2,2.6 \mathrm{~Hz}, 1 \mathrm{H}), 3.02(\mathrm{bs}, 1 \mathrm{H}), 1.85-1.62(\mathrm{~m}, 3 \mathrm{H})$, $1.16-1.04(\mathrm{~m}, 1 \mathrm{H}) .{ }^{13} \mathbf{C} \mathbf{N M R}\left(126 \mathbf{M H z}, \mathbf{C}_{6} \mathbf{D}_{6}\right) \delta 158.9,136.8,126.5,113.5,76.9,70.3,67.5$, 54.5, 35.5, 22.2. IR (neat): 3431, 2949, 2843, 1609, 1512, 1302, 1246, 1177, 1082, 1034, 1016, 916, 827, 793, $731 \mathrm{~cm}^{-1}$. HRMS (EI): exact mass calculated for $[\mathrm{M} \bullet]^{+}\left(\mathrm{C}_{12} \mathrm{H}_{16} \mathrm{O}_{3}\right)$ requires $\mathrm{m} / z$ 208.1094, found $\mathrm{m} / \mathrm{z} 208.1093$. 


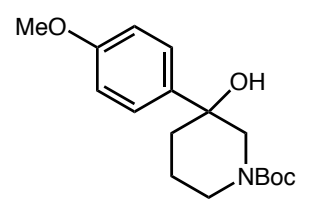

tert-butyl 3-hydroxy-3-(4-methoxyphenyl)piperidine-1-carboxylate

Prepared following the general procedure B with tert-butyl 3-oxopiperidine-1-carboxylate to afford the titled compound as a colorless oil (2.01 g, 43\%). ${ }^{1} \mathbf{H}$ NMR (500 $\left.\mathbf{~ M H z , ~} \mathbf{C}_{\mathbf{6}} \mathbf{D}_{\mathbf{6}}\right) \delta 7.33$ $(\mathrm{d}, J=8.6 \mathrm{~Hz}, 2 \mathrm{H}), 6.80(\mathrm{~d}, J=8.8 \mathrm{~Hz}, 2 \mathrm{H}), 4.24-3.96(\mathrm{~m}, 2 \mathrm{H}), 3.32(\mathrm{~s}, 3 \mathrm{H}), 2.97(\mathrm{~d}, J=13.6$ $\mathrm{Hz}, 1 \mathrm{H}), 2.56(\mathrm{t}, J=12.6 \mathrm{~Hz}, 1 \mathrm{H}), 1.76(\mathrm{t}, J=6.7 \mathrm{~Hz}, 1 \mathrm{H}), 1.61-1.49(\mathrm{~m}, 1 \mathrm{H}), 1.45(\mathrm{~s}, 9 \mathrm{H})$, $1.18-1.07(\mathrm{~m}, 2 \mathrm{H}) .{ }^{\mathbf{1 3}} \mathbf{C} \mathbf{N M R}\left(\mathbf{7 5} \mathbf{~ M H z}, \mathbf{C}_{\mathbf{6}} \mathbf{D}_{\mathbf{6}}, \mathbf{5 0}^{\mathbf{0}} \mathbf{C}\right) \delta 159.4,156.0,138.7,126.6,114.1,79.5$, 71.7, 55.4, 54.9, 44.5, 37.4, 28.6, 21.8. IR (neat): 3441, 2947, 1678, 1513, 1419, 1365, 1244, $1148,1035,827,733 \mathrm{~cm}^{-1}$. MS (ESI): exact mass calculated for $\left[\mathrm{M}-\mathrm{Boc}-\mathrm{H}_{2} \mathrm{O}+2 \mathrm{H}\right]^{+}\left(\mathrm{C}_{12} \mathrm{H}_{16} \mathrm{NO}\right)$ requires $m / z 190.1$, found $m / z 190.1$.

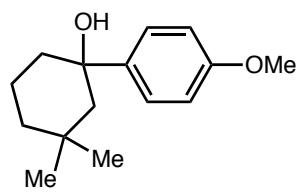

\section{1-(4-methoxyphenyl)-3,3-dimethylcyclohexan-1-ol}

Prepared following the general procedure A with 3,3-dimethylcyclohexan-1-one to afford the titled compound as a colorless oil $(0.74 \mathrm{~g}, 43 \%)$. ${ }^{1} \mathbf{H}$ NMR $\left(\mathbf{5 0 0} \mathbf{M H z}, \mathbf{C}_{6} \mathbf{D}_{6}\right) \delta 7.31(\mathrm{~d}, J=8.8$ $\mathrm{Hz}, 2 \mathrm{H}), 6.84(\mathrm{~d}, J=8.8 \mathrm{~Hz}, 2 \mathrm{H}), 3.36(\mathrm{~s}, 3 \mathrm{H}), 2.04-1.91(\mathrm{~m}, 1 \mathrm{H}), 1.65-1.39(\mathrm{~m}, 6 \mathrm{H}), 1.25(\mathrm{~s}$, $3 \mathrm{H}), 1.10(\mathrm{td}, J=13.3,12.2,4.0 \mathrm{~Hz}, 1 \mathrm{H}), 0.86(\mathrm{~s}, 3 \mathrm{H}) .{ }^{13} \mathbf{C} \mathbf{N M R}\left(\mathbf{1 2 6} \mathbf{M H z}, \mathbf{C}_{\mathbf{6}} \mathbf{D}_{\mathbf{6}}\right) \delta 158.5$, 142.7, 125.8, 113.3, 73.6, 54.5, 51.0, 38.8, 34.1, 31.0, 27.2, 19.0. IR (neat): 3476, 2996, 2940, 2865, 2839, 1610, 1510, 1458, 1298, 1179, 1036, 989, 825, $795 \mathrm{~cm}^{-1}$. HRMS (EI): exact mass calculated for $[\mathrm{M} \bullet]^{+}\left(\mathrm{C}_{15} \mathrm{H}_{22} \mathrm{O}_{2}\right)$ requires $\mathrm{m} / \mathrm{z} 234.1614$, found $\mathrm{m} / \mathrm{z} 234.1612$.

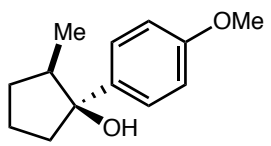

\section{1-(4-methoxyphenyl)-2-methylcyclopentanol}

Prepared following general procedure A with 2-methylcyclopentanone to afford the titled compound as a colorless oil as a single diastereomer (0.9 g, 29\%). Spectra are consistent with reported literature values. ${ }^{4}$

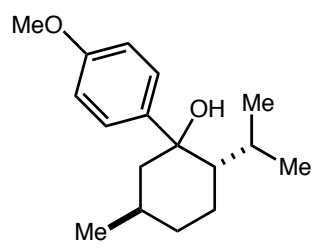

(2S,5R)-2-isopropyl-1-(4-methoxyphenyl)-5-methylcyclohexan-1-ol

Prepared following the general procedure B with menthone to afford the titled compound as a colorless oil as an $\sim 3: 1$ mixture of diastereomers $(1.3 \mathrm{~g}, 48 \%)$, which was carried on together to the next step without further separation. Characterization of this mixture is given herein. ${ }^{\mathbf{1}} \mathbf{H}$ NMR (500 MHz, CDCl $\left.{ }_{3}\right) \delta 7.35(\mathrm{~d}, J=8.9 \mathrm{~Hz}, 2 \mathrm{H}), 6.88(\mathrm{~d}, J=9.1 \mathrm{~Hz}, 2 \mathrm{H}), 3.81(\mathrm{~s}, 3 \mathrm{H}), 1.89$ $-1.84(\mathrm{~m}, 1 \mathrm{H}), 1.83-1.77(\mathrm{~m}, 1 \mathrm{H}), 1.68-1.59(\mathrm{~m}, 3 \mathrm{H}), 1.56-1.49(\mathrm{~m}, 1 \mathrm{H}), 1.45-1.39(\mathrm{~m}$, $1 \mathrm{H}), 1.23(\mathrm{~d}, J=7.3 \mathrm{~Hz}, 1 \mathrm{H}), 1.07-0.98(\mathrm{~m}, 1 \mathrm{H}), 0.89$ (d, $J=6.5 \mathrm{~Hz}, 3 \mathrm{H}), 0.82(\mathrm{~d}, J=6.9 \mathrm{~Hz}$, 
$3 \mathrm{H}), 0.73(\mathrm{~d}, J=6.9 \mathrm{~Hz}, 3 \mathrm{H}) .{ }^{13} \mathbf{C}$ NMR $\left(\mathbf{1 2 6} \mathbf{~ M H z}, \mathbf{C}_{\mathbf{6}} \mathbf{D}_{6}\right) \delta 158.3,140.9,125.8,113.4,77.6$, 54.4, 51.7, 50.2, 35.2, 28.2, 26.7, 23.6, 22.2, 21.2, 18.3. IR (neat): 3521, 2949, 2868, 1732, $1609,1509,1243,1175,1037,826 \mathrm{~cm}^{-1}$. MS (ESI): exact mass calculated for $\left[\mathrm{M}-\mathrm{H}_{2} \mathrm{O}+\mathrm{H}\right]^{+}$ $\left(\mathrm{C}_{17} \mathrm{H}_{25} \mathrm{O}\right)$ requires $\mathrm{m} / \mathrm{z} 245.2$, found $\mathrm{m} / \mathrm{z} 245.2$.

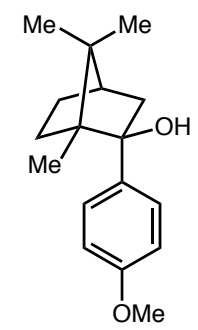

(1R,2S,4R)-2-(4-methoxyphenyl)-1,7,7-trimethylbicyclo[2.2.1] heptan-2-ol

The titled compound was prepared according to a literature procedure. ${ }^{5}$ Spectra are consistent with reported literature values.

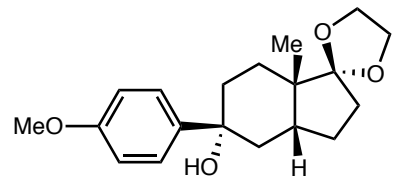

(3aR,7aS)-5-(4-methoxyphenyl)-7a-methyloctahydrospiro[indene-1,2'-[1,3]dioxolan]-5-ol Prepared following the general procedure $\mathrm{B}$ with $(3 \mathrm{a} R, 7 \mathrm{a} S)$-7a-methylhexahydrospiro[indene$1,2^{\prime}$-[1,3]dioxolan]-5(4H)-one (prepared according to a literature procedure ${ }^{6}$ ) to afford the titled compound as a colorless oil as a single diastereomer $(0.701 \mathrm{~g}, 45 \%) .{ }^{1} \mathbf{H} \mathbf{~ N M R}\left(\mathbf{5 0 0} \mathbf{~ M H z}, \mathbf{C}_{\mathbf{6}} \mathbf{D}_{\mathbf{6}}\right)$ $\delta 7.32(\mathrm{~d}, J=8.8 \mathrm{~Hz}, 2 \mathrm{H}), 6.84(\mathrm{~d}, J=8.8 \mathrm{~Hz}, 2 \mathrm{H}), 3.58-3.50(\mathrm{~m}, 4 \mathrm{H}), 3.36(\mathrm{~s}, 3 \mathrm{H}), 2.64-$ $2.54(\mathrm{~m}, 1 \mathrm{H}), 2.25(\mathrm{td}, J=12.1,11.1,5.0 \mathrm{~Hz}, 1 \mathrm{H}), 2.19-2.10(\mathrm{~m}, 2 \mathrm{H}), 2.04-1.93(\mathrm{~m}, 1 \mathrm{H})$, $1.90-1.80(\mathrm{~m}, 3 \mathrm{H}), 1.79-1.71(\mathrm{~m}, 1 \mathrm{H}), 1.63-1.48(\mathrm{~m}, 2 \mathrm{H}), 1.14(\mathrm{~s}, 3 \mathrm{H}) .{ }^{13} \mathbf{C}$ NMR (126 MHz, $\left.\mathbf{C}_{6} \mathbf{D}_{6}\right) \delta 158.6,142.6,125.8,120.5,113.4,72.9,64.9,63.8,54.5,44.1,41.5,37.4,34.3$, 32.9, 25.8, 24.8, 17.2. IR (neat): 3483, 2956, 1608, 1507, 1248, 1175, 1016, 1034, 947, $831 \mathrm{~cm}^{-}$ ${ }^{1}$. MS (ESI): exact mass calculated for $[\mathrm{M}+\mathrm{H}]^{+}\left(\mathrm{C}_{19} \mathrm{H}_{27} \mathrm{O}_{4}\right)$ requires $\mathrm{m} / \mathrm{z} 319.2$, found $\mathrm{m} / z 319.1$.

\section{2-(4-methoxyphenyl)-1-methylcyclohexan-1-ol}

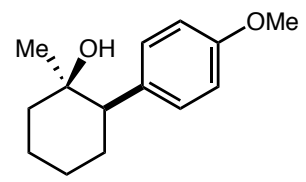

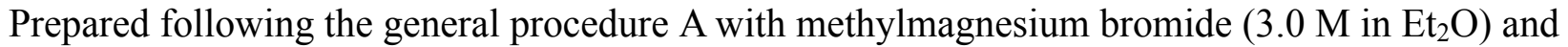
2-(4-methoxyphenyl)cyclohexan-1-one (racemic starting material prepared according to a literature procedure ${ }^{7}$ ) to afford the titled compound as a colorless oil as a single diastereomer $(0.59 \mathrm{~g}, 99 \%) .{ }^{1} \mathbf{H}$ NMR $\left(\mathbf{5 0 0} \mathbf{~ M H z}, \mathbf{C}_{6} \mathbf{D}_{\mathbf{6}}\right) \delta 7.14(\mathrm{~d}, J=8.6 \mathrm{~Hz}, 2 \mathrm{H}), 6.81(\mathrm{~d}, J=8.8 \mathrm{~Hz}, 2 \mathrm{H})$, $3.35(\mathrm{~s}, 3 \mathrm{H}), 2.24$ (dd, $J=12.9,3.4 \mathrm{~Hz}, 1 \mathrm{H}), 2.10(\mathrm{qd}, J=12.9,3.6 \mathrm{~Hz}, 1 \mathrm{H}), 1.86$ (qt, $J=13.4$, $3.8 \mathrm{~Hz}, 1 \mathrm{H}), 1.76(\mathrm{dq}, \mathrm{J}=13.0,2.9 \mathrm{~Hz}, 1 \mathrm{H}), 1.68(\mathrm{dt}, \mathrm{J}=12.2,3.4 \mathrm{~Hz}, 1 \mathrm{H}), 1.62-1.54(\mathrm{~m}, 1 \mathrm{H})$, $1.54-1.46(\mathrm{~m}, 1 \mathrm{H}), 1.31-1.15(\mathrm{~m}, 2 \mathrm{H}), 0.86(\mathrm{~s}, 3 \mathrm{H}), 0.82(\mathrm{~s}, 1 \mathrm{H}) .{ }^{13} \mathbf{C} \mathbf{~ N M R}\left(\mathbf{1 2 6} \mathbf{~ M H z}, \mathbf{C}_{6} \mathbf{D}_{6}\right)$ $\delta 158.5,135.2,129.9,113.4,70.1,54.4,52.3,40.3,29.9,29.0,26.7,22.0$. IR (neat): 3499, 2928 , $2856,1609,1510,1448,1243,1178,1035,826,800 \mathrm{~cm}^{-1}$. MS (ESI): exact mass for [M$\left.\mathrm{H}_{2} \mathrm{O}+\mathrm{H}\right]^{+}\left(\mathrm{C}_{14} \mathrm{H}_{19} \mathrm{O}\right)$ requires $m / z 203.1$, found $\mathrm{m} / z 203.1$. 


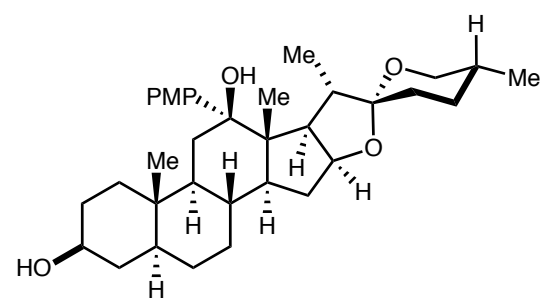

(2aS,4S,5'R,6aS,6bS,8aS,8bR,9S,10R,11aS,12aS)-8-(4-methoxyphenyl)-5',6a,8a,9tetramethyldocosahydrospiro[naphtho $\left[2^{\prime}, 1^{\prime}: 4,5\right]$ indeno $[2,1-b]$ furan-10,2'-pyran $]-4,8-d i o l$ Prepared following the general procedure B with hecogenin acetate (1 equiv), 1-iodo-4methoxybenzene ( 8 equiv), and $\mathrm{Mg}$ (8.4 equiv) to afford the titled compound as a white solid as a single diastereomer $(2.02 \mathrm{~g}, 89 \%)$. ${ }^{1} \mathbf{H}$ NMR (500 MHz, $\left.\mathbf{C}_{6} \mathbf{D}_{6}\right) \delta 7.43(\mathrm{~d}, J=8.8 \mathrm{~Hz}, 2 \mathrm{H}), 6.86$ $(\mathrm{d}, J=8.9 \mathrm{~Hz}, 2 \mathrm{H}), 4.69(\mathrm{ddd}, J=8.7,7.4,6.2 \mathrm{~Hz}, 1 \mathrm{H}), 3.59-3.51(\mathrm{~m}, 2 \mathrm{H}), 3.42-3.31(\mathrm{~m}$, $1 \mathrm{H}), 3.35(\mathrm{~s}, 3 \mathrm{H}), 2.93(\mathrm{dd}, J=8.7,6.6 \mathrm{~Hz}, 1 \mathrm{H}), 2.24(\mathrm{ddd}, J=14.0,10.9,6.0 \mathrm{~Hz}, 1 \mathrm{H}), 2.18-$ $2.05(\mathrm{~m}, 2 \mathrm{H}), 1.60-1.35(\mathrm{~m}, 11 \mathrm{H}), 1.33-1.24(\mathrm{~m}, 2 \mathrm{H}), 1.24-1.16(\mathrm{~m}, 2 \mathrm{H}), 1.16-1.07(\mathrm{~m}$, $3 \mathrm{H}), 1.03$ (ddd, $J=12.4,10.7,4.1 \mathrm{~Hz}, 1 \mathrm{H}), 0.95$ (s, 3H), 0.85 (d, $J=7.0 \mathrm{~Hz}, 3 \mathrm{H}), 0.73(\mathrm{~s}, 3 \mathrm{H})$, $0.69-0.61(\mathrm{~m}, 1 \mathrm{H}), 0.59(\mathrm{~d}, J=6.4 \mathrm{~Hz}, 4 \mathrm{H}), 0.51(\mathrm{bs}, 1 \mathrm{H}) .{ }^{13} \mathbf{C} \mathbf{N M R}\left(\mathbf{1 2 6} \mathbf{M H z}, \mathbf{C}_{6} \mathbf{D}_{6}\right) \delta$ $158.7,139.1,128.3,112.6,108.7,80.7,76.4,70.7,66.7,54.5,53.0,49.9,49.5,49.1,44.7,42.5$, $38.4,36.8,35.6,35.1,34.6,31.8,31.8,31.7,31.5,30.3,28.9,28.6,17.0,15.3,14.5,12.2$. IR (neat): $3424,2927,2859,1609,1510,1457,1296,1247,1179,1178,1044,981,899,865,832$, $737 \mathrm{~cm}^{-1}$. MS (ESI): exact mass calculated for $[\mathrm{M}+\mathrm{H}]^{+}\left(\mathrm{C}_{34} \mathrm{H}_{51} \mathrm{O}_{5}\right)$ requires $\mathrm{m} / z$ 539.4, found $\mathrm{m} / z$ 539.4 .

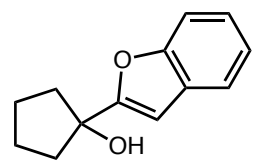

\section{1-(benzofuran-2-yl)cyclopentanol}

Prepared following general procedure $\mathrm{C}$ with benzofuran and cyclopentanone to afford the titled compound as a white solid (1.6 g, 54\%). ${ }^{1} \mathbf{H}$ NMR (500 MHz, $\left.\mathbf{C}_{6} \mathbf{D}_{6}\right) \delta 7.40(\mathrm{~d}, J=7.4 \mathrm{~Hz}, 1 \mathrm{H})$, $7.35(\mathrm{~d}, J=7.9 \mathrm{~Hz}, 1 \mathrm{H}), 7.12-7.05(\mathrm{~m}, 2 \mathrm{H}), 6.36(\mathrm{~s}, 1 \mathrm{H}), 2.06-1.98(\mathrm{~m}, 2 \mathrm{H}), 1.86-1.77(\mathrm{~m}$, 4H), $1.60-1.53$ (m, 2H), 1.28 (s, 1H). ${ }^{13}$ C NMR (126 MHz, C $\mathbf{D}_{6}$ ) $\delta 162.5,155.0,128.7,123.8$, 122.7, 120.8, 111.1, 101.0, 79.4, 39.8, 23.8. IR (neat): 3372, 2960, 2874, 1451, 1251, 1170, $1009,985,806,750,741 \mathrm{~cm}^{-1}$. MS (EI): exact mass calculated for $[\mathrm{M} \bullet]^{+}\left(\mathrm{C}_{13} \mathrm{H}_{14} \mathrm{O}_{2}\right)$ requires $\mathrm{m} / z$ 202.1, found $m / z$ 202.1. $\boldsymbol{E}_{\mathbf{p} / \mathbf{2}}\left(\mathbf{v s} . \mathbf{F c} / \mathbf{F c} \mathbf{c}^{+}\right)=1.27 \mathrm{~V}$.

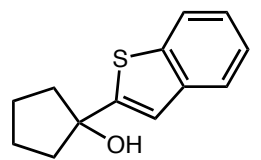

\section{1-(benzo $[b]$ thiophen-2-yl)cyclopentanol}

Prepared following general procedure $\mathrm{C}$ with benzo $[b]$ thiophene and cyclopentanone to afford the titled compound as a pale-yellow solid (1.9 g, 58\%). ${ }^{\mathbf{1}} \mathbf{H} \mathbf{N M R}\left(\mathbf{5 0 0} \mathbf{M H z}, \mathbf{C}_{\mathbf{6}} \mathbf{D}_{\mathbf{6}}\right) \delta 7.57-$ $7.54(\mathrm{~m}, 2 \mathrm{H}), 7.20-7.17(\mathrm{~m}, 1 \mathrm{H}), 7.08-7.05(\mathrm{~m}, 1 \mathrm{H}), 6.92(\mathrm{~s}, 1 \mathrm{H}), 1.91-1.78(\mathrm{~m}, 6 \mathrm{H}), 1.59-$ $1.53(\mathrm{~m}, 2 \mathrm{H}), 1.29$ (s, 1H). ${ }^{13} \mathbf{C}$ NMR (126 MHz, $\left.\mathbf{C}_{6} \mathbf{D}_{6}\right) \delta 153.5,140.1,139.6,124.1,123.8$, 123.2, 122.3, 118.5, 81.3, 42.5, 23.6. IR (neat): 3369, 2957, 2872, 1457, 1435, 1304, 1068, $1000,828,743,726 \mathrm{~cm}^{-1}$. HRMS (EI): exact mass calculated for $[\mathrm{M} \bullet]^{+}\left(\mathrm{C}_{13} \mathrm{H}_{14} \mathrm{OS}\right)$ requires $\mathrm{m} / z$ 218.0760, found $m / z$ 218.0758. $\boldsymbol{E}_{\mathbf{p} / 2}\left(\mathbf{v s} . \mathbf{F c} / \mathbf{F c}^{+}\right)=1.18 \mathrm{~V}$. 


\section{1-(naphthalen-2-yl)cyclopentanol}

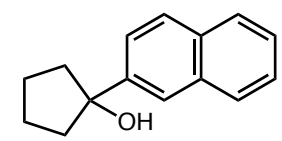

Prepared following general procedure $\mathrm{C}$ with 2-bromonaphthalene and cyclopentanone to afford the titled compound as a white solid (1.5 g, 35\%). ${ }^{\mathbf{1}} \mathbf{H} \mathbf{N M R}\left(\mathbf{5 0 0} \mathbf{M H z}, \mathbf{C}_{6} \mathbf{D}_{\mathbf{6}}\right) \delta 7.90(\mathrm{~s}, 1 \mathrm{H})$, $7.73(\mathrm{~d}, J=7.9 \mathrm{~Hz}, 1 \mathrm{H}), 7.67(\mathrm{t}, J=8.2 \mathrm{~Hz}, 2 \mathrm{H}), 7.45(\mathrm{~d}, J=8.7 \mathrm{~Hz}, 1 \mathrm{H}), 7.30$ (p, $J=7.0 \mathrm{~Hz}$, 2H), $1.97-1.79(\mathrm{~m}, 6 \mathrm{H}), 1.68-1.62(\mathrm{~m}, 2 \mathrm{H}), 0.99(\mathrm{~s}, 1 \mathrm{H}) .{ }^{13} \mathbf{C}$ NMR (126 MHz, C6 $\left.\mathbf{~ D} \mathbf{D}_{6}\right)$ $144.9,133.5,132.6,128.1,128.0,127.8,125.9,125.5,124.3,123.2,82.9,41.9,24.1$. IR (neat): 3380, 2957, 2871, 1350, 1274, 1193, 1004, 856, 816, $745 \mathrm{~cm}^{-1}$. MS (EI): exact mass calculated for $[\mathrm{M} \bullet]^{+}\left(\mathrm{C}_{15} \mathrm{H}_{16} \mathrm{O}\right)$ requires $m / z 212.1$, found $m / z 212.1 . \boldsymbol{E}_{\mathbf{p} / 2}\left(\mathbf{v s} . \mathbf{F c} / \mathbf{F c}{ }^{+}\right)=1.24 \mathrm{~V}$.

\section{1-(phenanthren-9-yl)cyclopentanol}

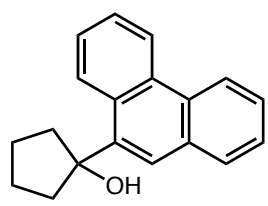

Prepared following general procedure $\mathrm{C}$ with 9-bromophenanthrene and cyclopentanone to afford the titled compound as a viscous oil (1.1 g, 41\%). ${ }^{1} \mathbf{H}$ NMR (500 MHz, $\left.\mathbf{C}_{6} \mathbf{D}_{6}\right) \delta 8.96(\mathrm{~d}, J$ $=8.5 \mathrm{~Hz}, 1 \mathrm{H}), 8.58(\mathrm{~d}, J=8.1 \mathrm{~Hz}, 1 \mathrm{H}), 8.49-8.47(\mathrm{~m}, 1 \mathrm{H}), 7.73-7.70(\mathrm{~m}, 1 \mathrm{H}), 7.66(\mathrm{~s}, 1 \mathrm{H})$, $7.50-7.41(\mathrm{~m}, 4 \mathrm{H}), 2.16-2.04(\mathrm{~m}, 4 \mathrm{H}), 2.00-1.91(\mathrm{~m}, 2 \mathrm{H}), 1.64-1.56(\mathrm{~m}, 2 \mathrm{H}), 1.24(\mathrm{~s}, 1 \mathrm{H})$. ${ }^{13} \mathbf{C}$ NMR (126 MHz, $\left.\mathbf{C}_{6} \mathbf{D}_{6}\right) \delta 140.3,131.7,131.4,131.2,130.5,128.8,128.6,126.6,126.5$, 125.9, 125.8, 123.6, 123.1, 122.5, 83.4, 40.4, 23.7. IR (neat): 3554, 3377, 2953, 2871, 1449, $1185,1000,889,767,746,726 \mathrm{~cm}^{-1}$. MS (EI): exact mass calculated for $[\mathrm{M} \bullet]^{+}\left(\mathrm{C}_{19} \mathrm{H}_{18} \mathrm{O}\right)$ requires $m / z 262.1$, found $m / z 262.1 . \boldsymbol{E}_{\mathbf{p} / 2}\left(\mathbf{v s} . \mathbf{F c} / \mathbf{F c}^{+}\right)=1.22 \mathrm{~V}$.

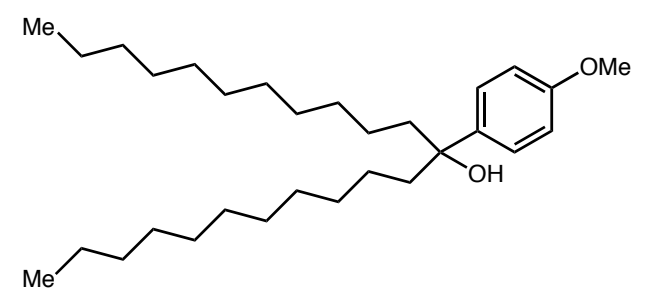

\section{2-(4-methoxyphenyl)tricosan-12-ol}

Prepared following general procedure A with tricosan-12-one to afford the titled compound as a colorless liquid (2.6 g, 38\%). ${ }^{1} \mathbf{H}$ NMR (500 MHz, $\left.\mathbf{C}_{6} \mathbf{D}_{6}\right) \delta 7.34(\mathrm{~d}, J=8.5 \mathrm{~Hz}, 2 \mathrm{H}), 6.86(\mathrm{~d}, J=$ $8.5 \mathrm{~Hz}, 2 \mathrm{H}), 3.33(\mathrm{~s}, 3 \mathrm{H}), 1.82-1.68(\mathrm{~m}, 4 \mathrm{H}), 1.43-1.16(\mathrm{~m}, 37 \mathrm{H}), 0.91(\mathrm{t}, J=6.9 \mathrm{~Hz}, 6 \mathrm{H})$. ${ }^{13}$ C NMR (126 MHz, C $\left.6 \mathbf{D}_{6}\right) \delta 158.3,138.7,126.5,113.4,76.2,54.4,43.6,32.0,30.3,29.79$, 29.76, 29.74, 29.5, 23.6, 22.8, 14.0. IR (neat): 3475, 2922, 2852, 1612, 1511, 1464, 1247, 1178, 1039, $830 \mathrm{~cm}^{-1}$. HRMS (EI): exact mass calculated for $\left[\mathrm{M}-\mathrm{H}_{2} \mathrm{O}\right]^{+}\left(\mathrm{C}_{30} \mathrm{H}_{52} \mathrm{O}\right)$ requires $\mathrm{m} / z$ 428.4013 , found $m / z 428.4023$.

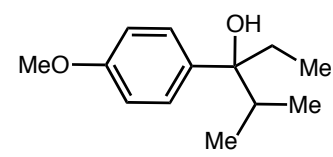

\section{3-(4-methoxyphenyl)-2-methylpentan-3-ol}

Prepared following general procedure $\mathrm{B}$ with 2-methylpentan-3-one to afford the titled compound as a colorless oil $(1.1 \mathrm{~g}, 55 \%)$. ${ }^{\mathbf{1}} \mathbf{H} \mathbf{N M R}\left(\mathbf{5 0 0} \mathbf{M H z}, \mathbf{C}_{6} \mathbf{D}_{6}\right) \delta 7.23(\mathrm{~d}, J=8.8 \mathrm{~Hz}, 2 \mathrm{H})$, 
$6.83(\mathrm{~d}, J=8.8 \mathrm{~Hz}, 2 \mathrm{H}), 3.34(\mathrm{~s}, 3 \mathrm{H}), 1.83(\mathrm{p}, J=6.8 \mathrm{~Hz}, 1 \mathrm{H}), 1.65$ (q, $J=7.4 \mathrm{~Hz}, 2 \mathrm{H}), 1.06(\mathrm{~s}$, $1 \mathrm{H}), 0.89(\mathrm{~d}, J=6.8 \mathrm{~Hz}, 3 \mathrm{H}), 0.74(\mathrm{~d}, J=6.9 \mathrm{~Hz}, 3 \mathrm{H}), 0.67(\mathrm{t}, J=7.4 \mathrm{~Hz}, 3 \mathrm{H}) .{ }^{13} \mathbf{C}$ NMR (126 MHz, $\left.\mathbf{C}_{6} \mathbf{D}_{6}\right) \delta 158.2,137.1,127.1,113.1,78.5,54.4,37.8,32.2,17.4,16.5,7.8$. IR (neat): 3513 , 2965, 2937, 2877, 1611, 1510, 1463, 1295, 1245, 1177, 1036, 973, $826 \mathrm{~cm}^{-1}$. MS (EI): exact mass calculated for $[\mathrm{M} \bullet]^{+}\left(\mathrm{C}_{13} \mathrm{H}_{20} \mathrm{O}_{2}\right)$ requires $m / z 208.1$, found $m / z 208.1$.

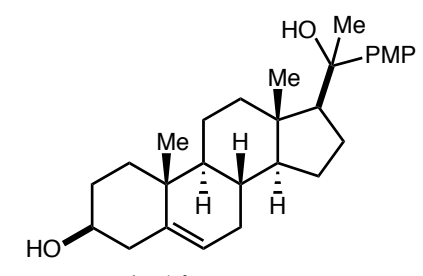

(3S,9S,10R,13S,14S,17S)-17-(1-hydroxy-1-(4-methoxyphenyl)ethyl)-10,13-dimethyl$\mathbf{2 , 3}, 4,7,8,9,10,11,12,13,14,15,16,17$-tetradecahydro- $1 H$-cyclopenta[ $a]$ phenanthren-3-ol

Prepared following the general procedure $\mathrm{C}$ with pregnenolone (1 equiv), 1-bromo-4methoxybenzene (4 equiv), and $t$-BuLi (4 equiv) to afford the titled compound as a white solid as an $\sim 2: 1$ mixture of diastereomers $(0.95 \mathrm{~g}, 35 \%)$. The mixture of diastereomers was carried on together to the next step (characterization is given for the major diastereomer, which was separated by preparative SFC). ${ }^{1} \mathbf{H}$ NMR (500 MHz, $\left.\mathbf{C}_{\mathbf{6}} \mathbf{D}_{\mathbf{6}}\right) \delta 7.35(\mathrm{~d}, J=8.8 \mathrm{~Hz}, 2 \mathrm{H}), 6.88(\mathrm{~d}, J$ $=8.8 \mathrm{~Hz}, 2 \mathrm{H}), 5.37-5.32(\mathrm{~m}, 1 \mathrm{H}), 3.37(\mathrm{~s}, 3 \mathrm{H}), 2.28-2.15(\mathrm{~m}, 2 \mathrm{H}), 1.98-1.89(\mathrm{~m}, 2 \mathrm{H}), 1.74$ - $1.49(\mathrm{~m}, 6 \mathrm{H}), 1.43-1.31(\mathrm{~m}, 2 \mathrm{H}), 1.29(\mathrm{~s}, 3 \mathrm{H}), 1.26(\mathrm{bs}, 1 \mathrm{H}), 1.19-1.02(\mathrm{~m}, 3 \mathrm{H}), 0.90(\mathrm{~s}$, $3 \mathrm{H}), 0.89-0.84(\mathrm{~m}, 2 \mathrm{H}), 0.82(\mathrm{~s}, 3 \mathrm{H}), 0.82-0.75(\mathrm{~m}, 2 \mathrm{H}), 0.67-0.53(\mathrm{~m}, 3 \mathrm{H}) .{ }^{13} \mathbf{C}$ NMR (126 MHz, $\left.\mathbf{C}_{6} \mathbf{D}_{6}\right) \delta 158.7,141.5,141.3,126.6,121.5,113.3,75.5,71.7,60.7,57.2,54.8,50.6,43.1$, 42.9, 39.3, 37.7, 36.8, 32.8, 32.3, 32.1, 31.7, 24.1, 23.3, 21.1, 19.5, 13.7. IR (neat): 3569, 3456, 2968, 2933, 2903, 2823, 1610, 1510, 1441, 1295, 1179, 1055, 1030, 839, 819, $736 \mathrm{~cm}^{-1}$. MS (ESI): exact mass calculated for $\left[\mathrm{M}-\mathrm{H}_{2} \mathrm{O}+\mathrm{H}\right]^{+}\left(\mathrm{C}_{28} \mathrm{H}_{39} \mathrm{O}_{2}\right)$ requires $m / z 407.3$, found $m / z$ 407.1.

\section{2-(4-methoxyphenyl)-1-phenylpropan-2-ol}

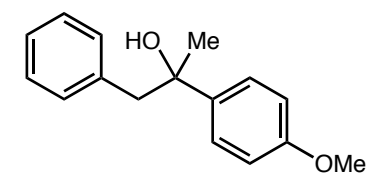

The titled compound was prepared according to a literature procedure, ${ }^{8}$ using 1-(4methoxyphenyl)ethan-1-one. ${ }^{1} \mathbf{H}$ NMR (500 MHz, $\left.\mathbf{C}_{6} \mathbf{D}_{6}\right) \delta 7.22(\mathrm{~d}, J=8.9 \mathrm{~Hz}, 2 \mathrm{H}), 7.10-7.01$ $(\mathrm{m}, 3 \mathrm{H}), 6.98-6.94(\mathrm{~m}, 2 \mathrm{H}), 6,79(\mathrm{~d}, J=8.9 \mathrm{~Hz}, 2 \mathrm{H}), 3.32(\mathrm{~s}, 3 \mathrm{H}), 2.98(\mathrm{~d}, J=13.2 \mathrm{~Hz}, 1 \mathrm{H})$, $2.89(\mathrm{~d}, J=13.2 \mathrm{~Hz}, 1 \mathrm{H}), 1.44(\mathrm{~s}, 1 \mathrm{H}), 1.37(\mathrm{~s}, 3 \mathrm{H}) .{ }^{13} \mathbf{C}$ NMR (126 MHz, $\left.\mathbf{C}_{6} \mathbf{D}_{6}\right) \delta 158.9,140.4$, 137.7, 131.0, 128.2, 126.7, 126.7, 113.6, 74.1, 54.8, 51.1, 29.7. IR (neat): 3455, 2971, 2932, 1611, 1511, 1454, 1298, 1247, 1178, 1090, 1032, 832, $703 \mathrm{~cm}^{-1}$. HRMS (EI): exact mass calculated for $\left[\mathrm{M}-\mathrm{H}_{2} \mathrm{O}\right]^{+}\left(\mathrm{C}_{16} \mathrm{H}_{16} \mathrm{O}\right)$ requires $m / z$ 224.1196, found $m / z$ 224.1199. $\boldsymbol{E}_{\mathbf{p} / 2}\left(\mathbf{v s} . \mathbf{F c} / \mathbf{F c}^{+}\right)$ $=1.14 \mathrm{~V}$.

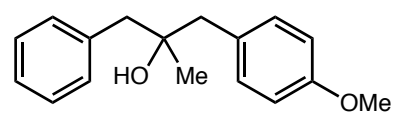

1-(4-methoxyphenyl)-2-methyl-3-phenylpropan-2-ol

The titled compound was prepared in an analogous fashion as the above compound, using 1-(4methoxyphenyl)propan-2-one. ${ }^{1} \mathbf{H}$ NMR (500 MHz, $\left.\mathbf{C}_{6} \mathbf{D}_{6}\right) \delta 7.18-7.13(\mathrm{~m}, 4 \mathrm{H}), 7.13-7.07$ $(\mathrm{m}, 1 \mathrm{H}), 7.04(\mathrm{~d}, J=8.6 \mathrm{~Hz}, 2 \mathrm{H}), 6.78$ (d, $J=8.6 \mathrm{~Hz}, 2 \mathrm{H}), 3.32$ (s, 3H), 2.69 (dd, $J=21.2,13.3$ 
$\mathrm{Hz}, 2 \mathrm{H}), 2.58(\mathrm{dd}, J=16.7,13.4 \mathrm{~Hz}, 2 \mathrm{H}), 0.91(\mathrm{~s}, 3 \mathrm{H}) .{ }^{\mathbf{1 3}} \mathbf{C} \mathbf{N M R}\left(\mathbf{1 2 6} \mathbf{M H z}, \mathbf{C}_{\mathbf{6}} \mathbf{D}_{\mathbf{6}}\right) \delta 158.6$, 138.0, 131.6, 130.8, 129.6, 128.2, 126.2, 113.5, 72.0, 54.4, 48.3, 47.5, 26.1. IR (neat): 3475, 3028, 2925, 2836, 1610, 1510, 1453, 1376, 1242, 1177, 1112, 1033, 832, 761, 735, $700 \mathrm{~cm}^{-1}$. HRMS (EI): exact mass calculated for $\left[\mathrm{M}-\mathrm{H}_{2} \mathrm{O}\right]^{+}\left(\mathrm{C}_{17} \mathrm{H}_{18} \mathrm{O}\right)$ requires $\mathrm{m} / z$ 238.1352, found $\mathrm{m} / \mathrm{z}$ 238.1353. $\boldsymbol{E}_{\mathbf{p} / 2}\left(\mathbf{v s} . \mathbf{F c} / \mathbf{F c}^{+}\right)=1.13 \mathrm{~V}$.

\section{4-(4-methoxyphenyl)-2-methyl-1-phenylbutan-2-ol}

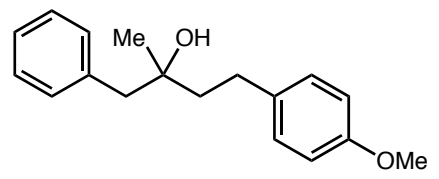

The titled compound was prepared in an analogous fashion as the above compound, using 4-(4methoxyphenyl)butan-2-one. ${ }^{1} \mathbf{H}$ NMR (500 MHz, $\left.\mathbf{C}_{6} \mathbf{D}_{6}\right) \delta 7.18-7.12(\mathrm{~m}, 4 \mathrm{H}), 7.11-7.06(\mathrm{~m}$, $1 \mathrm{H}), 7.01(\mathrm{~d}, J=8.5 \mathrm{~Hz}, 2 \mathrm{H}), 6.80(\mathrm{~d}, J=8.6 \mathrm{~Hz}, 2 \mathrm{H}), 3.34(\mathrm{~s}, 3 \mathrm{H}), 2.74-2.50(\mathrm{~m}, 4 \mathrm{H}), 1.73-$ 1.59 (m, 2H), 1.00 (s, 3H). ${ }^{13} \mathbf{C}$ NMR (126 MHz, $\left.\mathbf{C}_{6} \mathbf{D}_{6}\right) \delta 158.1,137.8,134.6,130.6,129.2$, 128.2, 126.3, 113.9, 71.7, 54.5, 48.2, 44.2, 29.5, 26.3. IR (neat): 3449, 3028, 2935, 2835, 1612, 1511, 1457, 1300, 1243, 1177, 1033, 821, 726, $701 \mathrm{~cm}^{-1}$. HRMS (EI): exact mass calculated for $\left[\mathrm{M}-\mathrm{H}_{2} \mathrm{O}\right]^{+}\left(\mathrm{C}_{18} \mathrm{H}_{20} \mathrm{O}\right)$ requires $m / z 252.1509$, found $\mathrm{m} / z$ 252.1511. $\boldsymbol{E}_{\mathbf{p} / 2}\left(\mathbf{v s} . \mathbf{F c} / \mathbf{F c}^{+}\right)=1.16 \mathrm{~V}$.

\section{5-(4-methoxyphenyl)-2-methyl-1-phenylpentan-2-ol}

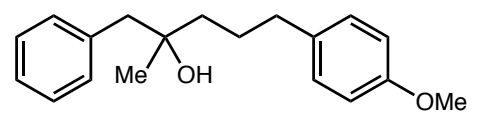

Prepared following the general procedure B with 1-phenylpropan-2-one (from oxidation of 1phenylpropan-2-ol) and 1-(3-bromopropyl)-4-methoxybenzene to afford the titled compound as a colorless oil (0.48 g, 48\%). ${ }^{1} \mathbf{H}$ NMR (500 MHz, $\left.\mathbf{C}_{6} \mathbf{D}_{6}\right) \delta 7.17-7.06(\mathrm{~m}, 5 \mathrm{H}), 7.01$ (d, $J=8.5$ $\mathrm{Hz}, 2 \mathrm{H}), 6.83(\mathrm{~d}, J=8.6 \mathrm{~Hz}, 2 \mathrm{H}), 3.35(\mathrm{~s}, 3 \mathrm{H}), 2.54(\mathrm{dd}, J=49.1,13.1 \mathrm{~Hz}, 2 \mathrm{H}), 2.44(\mathrm{t}, J=7.6$ $\mathrm{Hz}, 2 \mathrm{H}), 1.65$ (dddd, $J=22.5,13.0,9.4,5.5 \mathrm{~Hz}, 2 \mathrm{H}), 1.43-1.29(\mathrm{~m}, 2 \mathrm{H}), 0.93(\mathrm{~s}, 3 \mathrm{H}) .{ }^{13} \mathbf{C}$ NMR (126 MHz, $\left.\mathbf{C}_{6} \mathbf{D}_{6}\right) \delta 158.2,137.9,134.4,130.6,129.3,128.2,126.2,113.8,71.7,54.5$, 48.0, 41.4, 35.5, 26.4, 26.2. IR (neat): 3451, 3028, 2938, 1612, 1512, 1454, 1245, 1177, 1115, 1035, 927, 830, 729, $702 \mathrm{~cm}^{-1}$. HRMS (EI): exact mass calculated for $\left[\mathrm{M}-\mathrm{H}_{2} \mathrm{O}\right]^{+}\left(\mathrm{C}_{19} \mathrm{H}_{22} \mathrm{O}\right)$ requires $m / z 266.1665$, found $m / z 266.1665$. $\boldsymbol{E}_{\mathbf{p} / 2}\left(\mathbf{v s .} \mathbf{F c} / \mathbf{F c}^{+}\right)=1.16 \mathrm{~V}$.

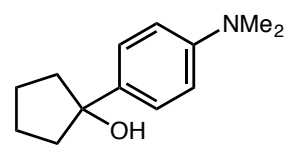

\section{1-(4-(dimethylamino)phenyl)cyclopentanol}

Prepared following general procedure A with 1-(4-(dimethylamino)phenyl)magnesium bromide ( $0.5 \mathrm{M}$ in THF, 2.1 equiv) and cylcopentanone to afford the titled compound as a pale-yellow powder (0.8 g, 66\%). ${ }^{1} \mathbf{H}$ NMR $\left(\mathbf{5 0 0} \mathbf{M H z}, \mathbf{C}_{\mathbf{6}} \mathbf{D}_{\mathbf{6}}\right) \delta 7.41(\mathrm{~d}, J=8.8 \mathrm{~Hz}, 2 \mathrm{H}), 6.66(\mathrm{~d}, J=8.8 \mathrm{~Hz}$, 2H), $2.56(\mathrm{~s}, 6 \mathrm{H}), 1.98-1.88(\mathrm{~m}, 6 \mathrm{H}), 1.69-1.62(\mathrm{~m}, 2 \mathrm{H}), 0.99(\mathrm{~s}, 1 \mathrm{H}) .{ }^{13} \mathbf{C}$ NMR (126 MHz, $\left.\mathbf{C}_{6} \mathbf{D}_{6}\right) \delta 149.6,135.7,126.0,112.5,82.6,41.5,40.1,23.8$. IR (neat): 3353, 2964, 2948, 2851, $1612,1521,1329,1208,1134,997,835,814 \mathrm{~cm}^{-1}$. MS (EI): exact mass calculated for $\left[\mathrm{M}-\mathrm{H}_{2} \mathrm{O}\right]^{+}$ $\left(\mathrm{C}_{13} \mathrm{H}_{17} \mathrm{~N}\right)$ requires $m / z$ 187.1, found $m / z$ 187.2. $\boldsymbol{E}_{\mathbf{p} / 2}\left(\mathbf{v s .} \mathbf{F c} / \mathbf{F c}^{+}\right)=0.39 \mathrm{~V}$. 


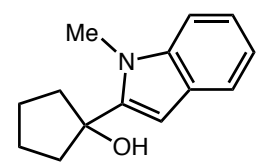

\section{1-(1-methyl-1 $H$-indol-2-yl)cyclopentanol}

Prepared following general procedure $\mathrm{C}$ with 1 -methyl- $1 H$-indole and cyclopentanone to afford the titled compound as a yellow solid $(0.7 \mathrm{~g}, 27 \%)$. ${ }^{1} \mathbf{H} \mathbf{N M R}\left(\mathbf{5 0 0} \mathbf{M H z}, \mathbf{C}_{\mathbf{6}} \mathbf{D}_{\mathbf{6}}\right) \delta 7.72(\mathrm{~d}, J=7.6$ $\mathrm{Hz}, 1 \mathrm{H}), 7.27(\mathrm{dt}, J=21.1,7.3 \mathrm{~Hz}, 2 \mathrm{H}), 7.13(\mathrm{~d}, J=7.9 \mathrm{~Hz}, 1 \mathrm{H}), 6.28(\mathrm{~s}, 1 \mathrm{H}), 3.49(\mathrm{~s}, 3 \mathrm{H}), 1.91$ - $1.76(\mathrm{~m}, 6 \mathrm{H}), 1.56-1.50(\mathrm{~m}, 2 \mathrm{H}), 0.74(\mathrm{~s}, 1 \mathrm{H}) .{ }^{13} \mathbf{C} \mathbf{N M R}\left(126 \mathbf{M H z}, \mathbf{C}_{6} \mathbf{D}_{6}\right) \delta 143.9,138.9$, $127.3,121.6,120.7,119.5,109.0,98.6,79.0,39.8,30.9,23.6$. IR (neat): 3378, 2951, 2872 , 1468, 1385, 1313, 993, 779, 749, $732 \mathrm{~cm}^{-1}$. HRMS (EI): exact mass calculated for [M•] ${ }^{+}$ $\left(\mathrm{C}_{14} \mathrm{H}_{17} \mathrm{NO}\right)$ requires $m / z 215.1305$, found $m / z 215.1301 . \boldsymbol{E}_{\mathbf{p} / 2}\left(\mathbf{v s . ~ F c} / \mathbf{F c}^{+}\right)=0.69 \mathrm{~V}$.

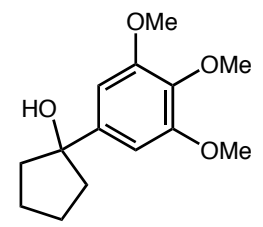

\section{1-(3,4,5-trimethoxyphenyl)cyclopentan-1-ol}

Prepared following the general procedure $\mathrm{C}$ with 5-bromo-1,2,3-trimethoxybenzene to afford the titled compound as an off-white solid (1.6 g, 78\%). ${ }^{1} \mathbf{H}$ NMR (500 MHz, $\left.\mathbf{C}_{6} \mathbf{D}_{6}\right) \delta 6.73(\mathrm{~s}, 2 \mathrm{H})$, $3.87(\mathrm{~s}, 3 \mathrm{H}), 3.48(\mathrm{~s}, 6 \mathrm{H}), 1.98-1.88(\mathrm{~m}, 2 \mathrm{H}), 1.88-1.79(\mathrm{~m}, 4 \mathrm{H}), 1.72-1.60(\mathrm{~m}, 2 \mathrm{H}) .{ }^{13} \mathbf{C}$ NMR (126 MHz, $\left.\mathbf{C}_{6} \mathbf{D}_{6}\right) \delta 153.5,142.9,138.1,103.3,83.0,60.2,55.7,42.0,23.9$. IR (neat): 3440, 2945, 2872, 2836, 1587, 1509, 1451, 1410, 1236, 1003, 831, 731, $652 \mathrm{~cm}^{-1}$. HRMS (EI): exact mass calculated for $[\mathrm{M} \bullet]^{+}\left(\mathrm{C}_{14} \mathrm{H}_{20} \mathrm{O}_{4}\right)$ requires $m / z$ 252.1356, found $m / z$ 252.1352. $\boldsymbol{E}_{\mathbf{p} / 2}$ (vs. $\left.\mathbf{F c} / \mathbf{F c}^{+}\right)=0.92 \mathrm{~V}$.

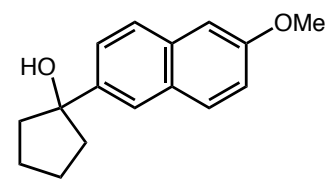

\section{1-(6-methoxynaphthalen-2-yl)cyclopentan-1-ol}

Prepared following the general procedure $\mathrm{C}$ with 2-bromo-6-methoxynaphthalene to afford the titled compound as a white solid (1.5 g, 73\%). ${ }^{\mathbf{1}} \mathbf{H} \mathbf{N M R}\left(\mathbf{5 0 0} \mathbf{M H z}, \mathbf{C}_{\mathbf{6}} \mathbf{D}_{6}\right) \delta 7.84(\mathrm{~d}, J=1.8 \mathrm{~Hz}$, $1 \mathrm{H}), 7.64(\mathrm{~d}, J=8.5 \mathrm{~Hz}, 1 \mathrm{H}), 7.59(\mathrm{~d}, J=8.9 \mathrm{~Hz}, 1 \mathrm{H}), 7.52(\mathrm{dd}, J=8.5,1.9 \mathrm{~Hz}, 1 \mathrm{H}), 7.24(\mathrm{dd}, J$ $=8.9,2.5 \mathrm{~Hz}, 1 \mathrm{H}), 6.99(\mathrm{~d}, J=2.4 \mathrm{~Hz}, 1 \mathrm{H}), 3.41(\mathrm{~s}, 3 \mathrm{H}), 2.02-1.81(\mathrm{~m}, 4 \mathrm{H}), 1.72-1.62(\mathrm{~m}$, 2H). ${ }^{13}$ C NMR (126 MHz, C $\left.\mathbf{6}_{6} \mathbf{D}_{6}\right) \delta 157.8,142.6,133.8,129.6,128.9,126.7,124.8,123.3,119.0$, 105.6, 82.9, 54.5, 41.8, 24.0. IR (neat): 3443, 3052, 3007, 2953, 2870, 2843, 1603, 1484, 1387, $1265,1202,1028,986,854,814,671 \mathrm{~cm}^{-1}$. HRMS (EI): exact mass calculated for $\left[\mathrm{M}-\mathrm{H}_{2} \mathrm{O}\right]^{+}$ $\left(\mathrm{C}_{16} \mathrm{H}_{16} \mathrm{O}\right)$ requires $m / z 224.1196$, found $m / z$ 224.1196. $\boldsymbol{E}_{\mathbf{p} / \mathbf{2}}\left(\mathbf{v s} . \mathbf{F c} / \mathbf{F c}^{+}\right)=0.96 \mathrm{~V}$. 


\section{Synthesis of Products}

\section{General procedure:}

A screw cap culture tube $(16 \times 125 \mathrm{~mm})$ outfitted with a PTFE/silicone septa was charged with the relevant alcohol (1 mmol, 1 equiv), ${ }^{9}\left[\operatorname{Ir}\left(\mathrm{dF}\left(\mathrm{CF}_{3}\right) \text { ppy }\right)_{2}\left(5,5^{\prime} \mathrm{d}\left(\mathrm{CF}_{3}\right)\right.\right.$ bpy $\left.)\right]\left(\mathrm{PF}_{6}\right)$ ( $0.01 \mathrm{mmol}, 0.01$ equiv, $11 \mathrm{mg}$ ) and redistilled collidine ( $3 \mathrm{mmol}, 3$ equiv, $396 \mu \mathrm{L})$. The sealed culture tube was then evacuated and backfilled with argon three times. Redistilled thiophenol $(0.25 \mathrm{mmol}, 0.25$ equiv, $26 \mu \mathrm{L})$ and $10 \mathrm{~mL}$ of degassed anhydrous $\mathrm{CH}_{2} \mathrm{Cl}_{2}$ were added via syringe. The reaction was irradiated by four blue LED lamps (Kessil H150B LED Grow Light), and let stir under an inert atmosphere at room temperature with a fan to cool the reaction setup. After 12-24 hours, the reaction was concentrated then purified by flash column chromatography (hexanes/EtOAc) to obtain the product. All scale-ups were run in duplicates and average yields are reported.
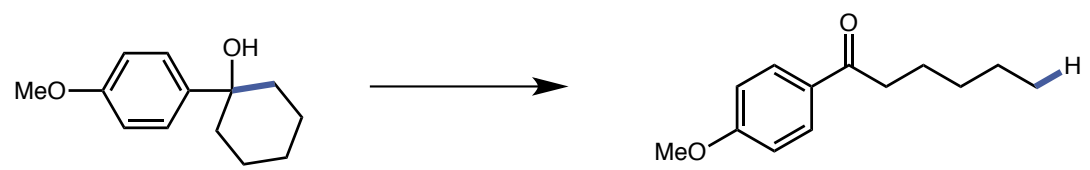

or<smiles>COc1ccc([C@]2(O)CCCC2C)cc1</smiles><smiles>COc1ccc(C(=O)CCCC(C)C)cc1</smiles>

\section{1-(4-methoxyphenyl)hexan-1-one}

Prepared on $1 \mathrm{mmol}$ scale following the general procedure with 1-(4methoxyphenyl)cyclohexan-1-ol. The crude material was purified by silica gel column chromatography to afford the titled compound as a pale yellow solid (183 $\mathrm{mg}, 89 \%)$.

Alternatively, prepared on $1 \mathrm{mmol}$ scale following the general procedure with 1-(4methoxyphenyl)-2-methylcyclopentanol. The crude is purified by silica gel column chromatography to afford the titled compound as a pale yellow oil (201 $\mathrm{mg}, 97 \%)$.

${ }^{1}$ H NMR (500 MHz, C $\left.\mathbf{~ D}_{6}\right) \delta 7.89(\mathrm{~d}, J=8.8 \mathrm{~Hz}, 2 \mathrm{H}), 6.66(\mathrm{~d}, J=8.9 \mathrm{~Hz}, 2 \mathrm{H}), 3.18(\mathrm{~s}, 3 \mathrm{H})$, $2.58(\mathrm{t}, J=7.3 \mathrm{~Hz}, 2 \mathrm{H}), 1.73(\mathrm{p}, J=7.3 \mathrm{~Hz}, 2 \mathrm{H}), 1.26-1.23(\mathrm{~m}, 4 \mathrm{H}), 0.84(\mathrm{t}, J=6.9 \mathrm{~Hz}, 3 \mathrm{H}) .{ }^{13} \mathbf{C}$ NMR (126 MHz, $\left.\mathbf{C}_{6} \mathbf{D}_{6}\right) \delta$ 197.2, 163.1, 130.6, 130.2, 113.5, 54.5, 37.9, 31.6, 24.1, 22.6, 13.9. IR (neat): 2956, 2933, 1676, 1600, 1510, 1254, 1170, 1031, $830 \mathrm{~cm}^{-1}$. HRMS (ESI): exact mass calculated for $[\mathrm{M}+\mathrm{H}]^{+}\left(\mathrm{C}_{13} \mathrm{H}_{19} \mathrm{O}_{2}\right)$ requires $m / z$ 207.13796, found $m / z$ 207.13784.
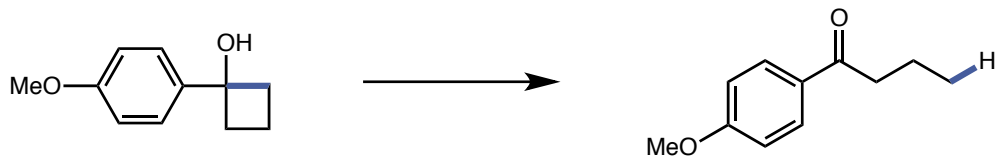

\section{1-(4-methoxyphenyl)butan-1-one}

Prepared on $1 \mathrm{mmol}$ scale following the general procedure with 1-(4-methoxyphenyl)cyclobutan1-ol. The crude material was purified by silica gel column chromatography to afford the titled compound as a pale yellow oil (126 mg, 71\%). ${ }^{1} \mathbf{H}$ NMR (500 MHz, $\left.\mathbf{C}_{6} \mathbf{D}_{6}\right) \delta 7.86(\mathrm{~d}, J=8.9 \mathrm{~Hz}$, $2 \mathrm{H}), 6.65(\mathrm{~d}, J=8.9 \mathrm{~Hz}, 2 \mathrm{H}), 3.19(\mathrm{~s}, 3 \mathrm{H}), 2.52(\mathrm{t}, J=7.2 \mathrm{~Hz}, 2 \mathrm{H}), 1.72(\mathrm{~h}, J=7.4 \mathrm{~Hz}, 2 \mathrm{H})$, 0.87 (t, $J=7.4 \mathrm{~Hz}, 3 \mathrm{H}) .{ }^{13} \mathbf{C}$ NMR (126 MHz, $\left.\mathbf{C}_{6} \mathbf{D}_{6}\right) \delta 197.0,163.1,130.6,130.1,113.5,54.5$, 
39.8, 17.7, 13.7. IR (neat): 2963, 1676, 1600, 1258, 1170, 1030, $832 \mathrm{~cm}^{-1}$. HRMS (ESI): exact mass calculated for $[\mathrm{M}+\mathrm{H}]^{+}\left(\mathrm{C}_{11} \mathrm{H}_{15} \mathrm{O}_{2}\right)$ requires $m / z$ 179.10666, found $m / z 179.10692$.
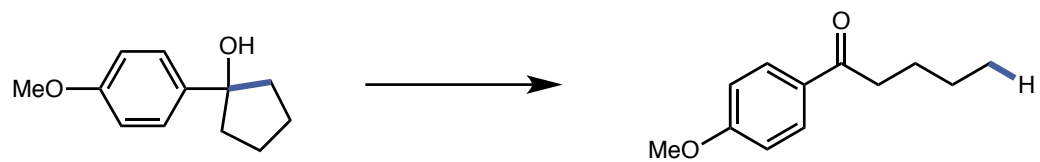

\section{1-(4-methoxyphenyl)pentan-1-one}

Prepared on $1 \mathrm{mmol}$ scale following the general procedure with 1-(4methoxyphenyl)cyclopentan-1-ol. The crude material was purified by silica gel column chromatography to afford the titled compound as a pale yellow oil (166 mg, 86\%). ${ }^{1} \mathbf{H}$ NMR $\left(\mathbf{5 0 0} \mathbf{M H z}, \mathbf{C}_{\mathbf{6}} \mathbf{D}_{6}\right) \delta 7.86(\mathrm{~d}, J=8.9 \mathrm{~Hz}, 2 \mathrm{H}), 6.67(\mathrm{~d}, J=8.8 \mathrm{~Hz}, 2 \mathrm{H}), 3.24(\mathrm{~s}, 3 \mathrm{H}), 2.58(\mathrm{t}, J=$ $7.4 \mathrm{~Hz}, 2 \mathrm{H}), 1.68(\mathrm{p}, J=7.4 \mathrm{~Hz}, 2 \mathrm{H}), 1.26(\mathrm{~h}, J=7.4 \mathrm{~Hz}, 2 \mathrm{H}), 0.84(\mathrm{t}, J=7.4 \mathrm{~Hz}, 3 \mathrm{H}) .{ }^{13} \mathrm{C}$ NMR (126 MHz, $\left.\mathbf{C}_{6} \mathbf{D}_{6}\right) \delta 197.2,163.1,130.5,130.1,113.5,54.6,37.6,26.5,22.5,13.8$. IR (neat): 2958, 2934, 2872, 1676, 1600, 1255, 1170, 1030, $840 \mathrm{~cm}^{-1}$. HRMS (ESI): exact mass calculated for $[\mathrm{M}+\mathrm{H}]^{+}\left(\mathrm{C}_{12} \mathrm{H}_{17} \mathrm{O}_{2}\right)$ requires $m / z$ 193.12231, found $m / z$ 193.12267.
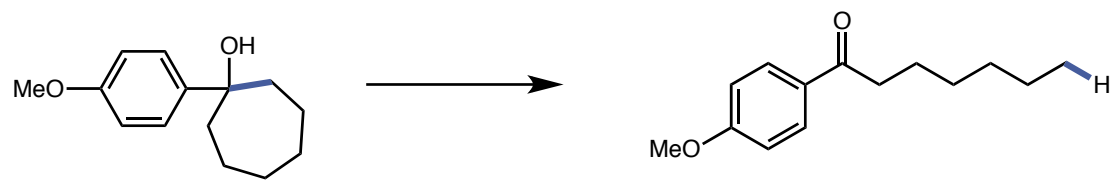

\section{1-(4-methoxyphenyl)heptan-1-one}

Prepared on $1 \mathrm{mmol}$ scale following the general procedure with 1-(4methoxyphenyl)cycloheptan-1-ol. The crude material was purified by silica gel column chromatography to afford the titled compound as a pale yellow solid (199 mg, 90\%). ${ }^{1} \mathbf{H}$ NMR $\left(\mathbf{5 0 0} \mathbf{M H z}, \mathbf{C}_{\mathbf{6}} \mathbf{D}_{\mathbf{6}}\right) \delta 7.88(\mathrm{~d}, J=8.9 \mathrm{~Hz}, 2 \mathrm{H}), 6.67(\mathrm{~d}, J=8.9 \mathrm{~Hz}, 2 \mathrm{H}), 3.24(\mathrm{~s}, 3 \mathrm{H}), 2.61(\mathrm{t}, J=$ $7.3 \mathrm{~Hz}, 2 \mathrm{H}), 1.71(\mathrm{p}, J=7.4 \mathrm{~Hz}, 2 \mathrm{H}), 1.35-1.09(\mathrm{~m}, 6 \mathrm{H}), 0.86(\mathrm{t}, J=7.0 \mathrm{~Hz}, 3 \mathrm{H}) .{ }^{13} \mathbf{C} \mathbf{N M R}$ $\left(\mathbf{1 2 6} \mathbf{M H z}, \mathbf{C}_{6} \mathbf{D}_{6}\right) \delta 197.2,163.1,130.6,130.1,113.5,54.6,37.9,31.8,29.1,24.4,22.6,14.0$. IR (neat): $3004,2950,2933,2858,1670,1604,1509,1407,1249,1179,1035,837 \mathrm{~cm}^{-1}$. HRMS (ESI): exact mass calculated for $[\mathrm{M}+\mathrm{H}]^{+}\left(\mathrm{C}_{14} \mathrm{H}_{21} \mathrm{O}_{2}\right)$ requires $\mathrm{m} / z$ 221.15361, found $\mathrm{m} / \mathrm{z}$ 221.15353 .
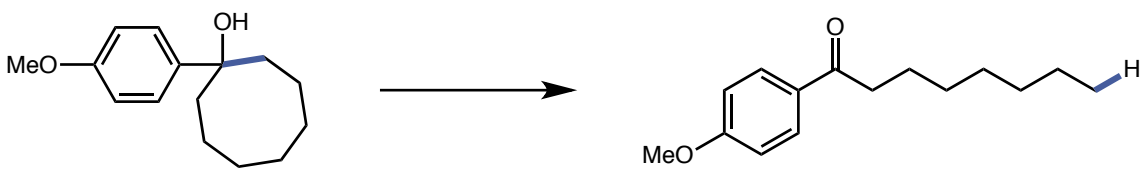

\section{1-(4-methoxyphenyl)octan-1-one}

Prepared on $1 \mathrm{mmol}$ scale following the general procedure with 1-(4-methoxyphenyl)cyclooctan1-ol. The crude material was purified by silica gel column chromatography to afford the titled compound as a pale yellow solid (189 mg, 81\%). $\left.{ }^{\mathbf{1}} \mathbf{H} \mathbf{~ N M R ~ ( 5 0 0 ~ M H z , ~} \mathbf{C}_{6} \mathbf{D}_{6}\right) \delta 7.90(\mathrm{~d}, J=8.9$ $\mathrm{Hz}, 2 \mathrm{H}), 6.66(\mathrm{~d}, J=8.8 \mathrm{~Hz}, 2 \mathrm{H}), 3.19(\mathrm{~s}, 3 \mathrm{H}), 2.61$ (t, $J=7.3 \mathrm{~Hz}, 2 \mathrm{H}), 1.75$ (p, $J=7.3 \mathrm{~Hz}, 2 \mathrm{H})$, $1.42-1.09(\mathrm{~m}, 8 \mathrm{H}), 0.88(\mathrm{t}, J=7.0 \mathrm{~Hz}, 3 \mathrm{H}) .{ }^{13} \mathbf{C}$ NMR (126 MHz, $\left.\mathbf{C}_{6} \mathbf{D}_{6}\right) \delta 197.2,163.1,130.6$, 130.2, 113.5, 54.5, 38.0, 31.8, 29.4, 29.3, 24.5, 22.7, 14.0. IR (neat): 2927, 2855, 1676, 1600, $1509,1256,1169,1032,812 \mathrm{~cm}^{-1}$. HRMS (ESI): exact mass calculated for $[\mathrm{M}+\mathrm{H}]^{+}\left(\mathrm{C}_{15} \mathrm{H}_{23} \mathrm{O}_{2}\right)$ requires $\mathrm{m} / \mathrm{z} 235.16926$, found $\mathrm{m} / \mathrm{z} 235.16923$. 

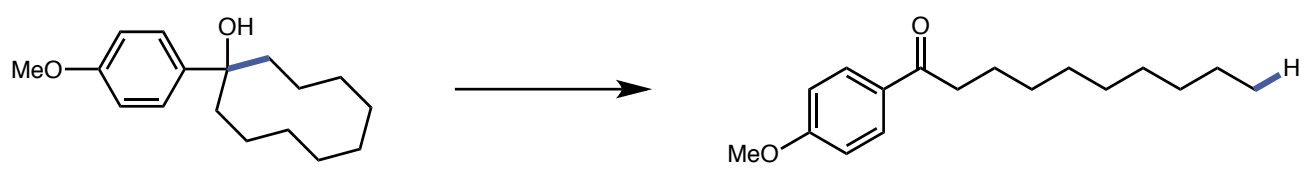

\section{1-(4-methoxyphenyl)decan-1-one}

Prepared on $1 \mathrm{mmol}$ scale following the general procedure with 1-(4methoxyphenyl)cyclodecan-1-ol. The crude material was purified by silica gel column chromatography to afford the titled compound as a pale yellow solid (241 mg, 92\%). ${ }^{1} \mathbf{H}$ NMR $\left(\mathbf{5 0 0} \mathbf{M H z}, \mathbf{C}_{6} \mathbf{D}_{\mathbf{6}}\right) \delta 7.90(\mathrm{~d}, J=8.9 \mathrm{~Hz}, 2 \mathrm{H}), 6.66(\mathrm{~d}, J=8.9 \mathrm{~Hz}, 2 \mathrm{H}), 3.19(\mathrm{~s}, 3 \mathrm{H}), 2.63(\mathrm{t}, J=$ $7.3 \mathrm{~Hz}, 2 \mathrm{H}), 1.77$ (p, $J=7.3 \mathrm{~Hz}, 2 \mathrm{H}), 1.26(\mathrm{~d}, J=9.8 \mathrm{~Hz}, 12 \mathrm{H}), 0.91(\mathrm{t}, J=7.0 \mathrm{~Hz}, 3 \mathrm{H}) .{ }^{13} \mathbf{C}$ NMR (126 MHz, $\left.\mathbf{C}_{6} \mathbf{D}_{6}\right) \delta 197.2,163.1,130.6,130.2,113.5,54.5,38.0,32.0,29.7,29.6,29.5$, 29.4, 24.5, 22.8, 14.0. IR (neat): 2954, 2915, 2849, 1678, 1605, 1579, 1254, 1178, 1031, 842, $811 \mathrm{~cm}^{-1}$. HRMS (ESI): exact mass calculated for $[\mathrm{M}+\mathrm{H}]^{+}\left(\mathrm{C}_{17} \mathrm{H}_{27} \mathrm{O}_{2}\right)$ requires $\mathrm{m} / \mathrm{z}$ 263.20056, found $m / z 263.20044$.

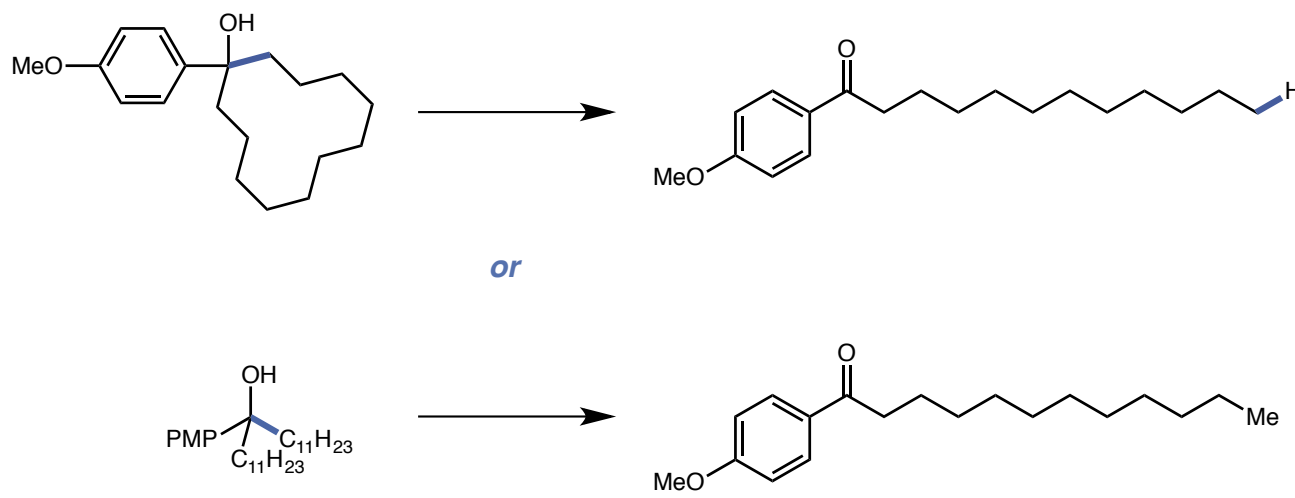

\section{1-(4-methoxyphenyl)dodecan-1-one}

Prepared on 1 mmol scale following the general procedure with 1-(4methoxyphenyl)cyclododec-1-ol. The crude material was purified by silica gel column chromatography to afford the titled compound as a pale yellow solid (245 mg, 85\%).

Alternatively, prepared on $1 \mathrm{mmol}$ scale following the general procedure with 12-(4methoxyphenyl)tricosan-12-ol. The crude is purified by silica gel column chromatography to afford the titled compound as a pale yellow powder (243 mg, 84\%). ${ }^{1} \mathbf{H} \mathbf{~ N M R}\left(\mathbf{5 0 0} \mathbf{~ M H z}, \mathbf{C}_{6} \mathbf{D}_{\mathbf{6}}\right.$ ) $\delta 7.91(\mathrm{~d}, J=8.7 \mathrm{~Hz}, 2 \mathrm{H}), 6.66(\mathrm{~d}, J=8.7 \mathrm{~Hz}, 2 \mathrm{H}), 3.18(\mathrm{~s}, 3 \mathrm{H}), 2.63(\mathrm{t}, J=7.3 \mathrm{~Hz}, 2 \mathrm{H}), 1.78$ $(\mathrm{p}, J=7.2 \mathrm{~Hz}, 2 \mathrm{H}), 1.33-1.28(\mathrm{~m}, 16 \mathrm{H}), 0.91(\mathrm{t}, J=6.8 \mathrm{~Hz}, 3 \mathrm{H}) .{ }^{13} \mathbf{C}$ NMR $\left(\mathbf{1 2 6} \mathbf{M H z}, \mathbf{C}_{\mathbf{6}} \mathbf{D}_{\mathbf{6}}\right) \delta$ 197.2, 163.1, 130.7, 130.2, 113.5, 54.5, 38.0, 32.0, 29.77, 29.75, 29.68, 29.5, 24.5, 22.8, 14.0. IR (neat): $2954,2915,2849,1678,1606,1580,1464,1256,1179,1031,841,810 \mathrm{~cm}^{-1}$. HRMS (ESI): exact mass calculated for $[\mathrm{M}+\mathrm{H}]^{+}\left(\mathrm{C}_{19} \mathrm{H}_{31} \mathrm{O}_{2}\right)$ requires $\mathrm{m} / \mathrm{z}$ 291.23186, found $\mathrm{m} / \mathrm{z}$ 291.23176 .
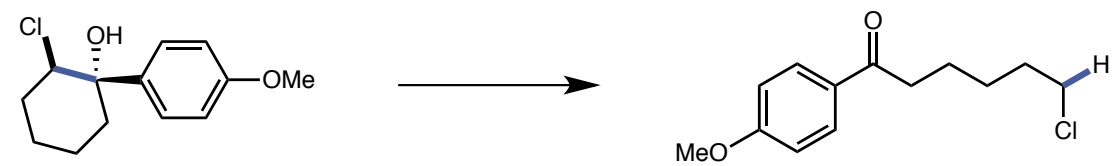

\section{6-chloro-1-(4-methoxyphenyl)hexan-1-one}

Prepared on $1 \mathrm{mmol}$ scale following the general procedure with 2-chloro-1-(4methoxyphenyl)cyclohexan-1-ol. The product was purified by silica gel column chromatography to afford the titled compound as a colorless oil (181 mg, 75\%). $\left.{ }^{\mathbf{1}} \mathbf{H} \mathbf{~ N M R ~ ( 5 0 0 ~} \mathbf{M H z}, \mathbf{C}_{\mathbf{6}} \mathbf{D}_{\mathbf{6}}\right) \delta$ $7.85(\mathrm{~d}, J=8.9 \mathrm{~Hz}, 2 \mathrm{H}), 6.68(\mathrm{~d}, J=8.9 \mathrm{~Hz}, 2 \mathrm{H}), 3.24$ (s, 3H), 3.10 (t, $J=6.7 \mathrm{~Hz}, 2 \mathrm{H}), 2.49$ (t, $J$ 
$=7.3 \mathrm{~Hz}, 2 \mathrm{H}), 1.54(\mathrm{dt}, J=15.1,7.3 \mathrm{~Hz}, 2 \mathrm{H}), 1.42(\mathrm{dq}, J=9.1,6.8 \mathrm{~Hz}, 2 \mathrm{H}), 1.25-1.13(\mathrm{~m}$, 2H). ${ }^{13}$ C NMR (126 MHz, $\left.\mathbf{C}_{6} \mathbf{D}_{6}\right) \delta 196.9,163.2,130.4,130.1,113.6,54.6,44.5,37.6,32.4$, 26.5, 23.4. IR (neat): 2938, 2865, 1674, 1599, 1575, 1509, 1418, 1309, 1256, 1169, 1029, 970, 834, 811, $718 \mathrm{~cm}^{-1}$. HRMS (ESI): exact mass calculated for $[\mathrm{M}+\mathrm{H}]^{+}\left(\mathrm{C}_{13} \mathrm{H}_{18} \mathrm{ClO}_{2}\right)$ requires $\mathrm{m} / z$ 241.09899 , found $\mathrm{m} / \mathrm{z} 241.09935$.
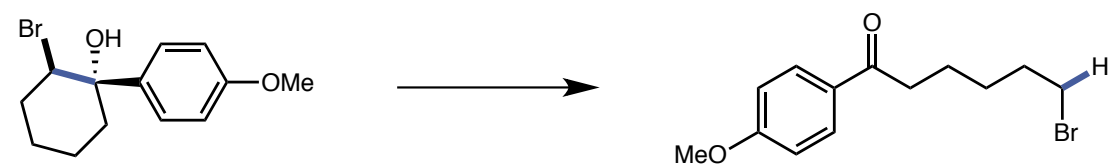

\section{6-bromo-1-(4-methoxyphenyl)hexan-1-one}

Prepared on $1 \mathrm{mmol}$ scale following the general procedure with 2-bromo-1-(4methoxyphenyl)cyclohexan-1-ol. The product was purified by silica gel column chromatography to afford the titled compound as a colorless oil (179 mg, 63\%). ${ }^{1} \mathbf{H}$ NMR (500 MHz, $\left.\mathbf{C}_{6} \mathbf{D}_{6}\right) \delta$ $7.85(\mathrm{~d}, J=8.9 \mathrm{~Hz}, 2 \mathrm{H}), 6.68(\mathrm{~d}, J=8.9 \mathrm{~Hz}, 2 \mathrm{H}), 3.23(\mathrm{~s}, 3 \mathrm{H}), 2.94(\mathrm{t}, J=6.8 \mathrm{~Hz}, 2 \mathrm{H}), 2.47(\mathrm{t}, J$ $=7.2 \mathrm{~Hz}, 2 \mathrm{H}), 1.58-1.44(\mathrm{~m}, 4 \mathrm{H}), 1.17(\mathrm{tdd}, J=9.9,8.4,4.7 \mathrm{~Hz}, 2 \mathrm{H}) .{ }^{13} \mathbf{C}$ NMR (126 MHz, $\left.\mathbf{C}_{6} \mathbf{D}_{6}\right) \delta 196.8,163.2,130.4,130.1,113.6,54.6,37.5,33.3,32.6,27.7,23.3$. IR (neat): 2936, 2839, 1673, 1598, 1575, 1509, 1418, 1362, 1252, 1217, 1168, 1028, 832, 810, $734 \mathrm{~cm}^{-1}$. HRMS (ESI): exact mass calculated for $[\mathrm{M}+\mathrm{H}]^{+}\left(\mathrm{C}_{13} \mathrm{H}_{18} \mathrm{BrO}_{2}\right)$ requires $\mathrm{m} / \mathrm{z} 285.04847$, found $\mathrm{m} / \mathrm{z}$ 285.04882 .
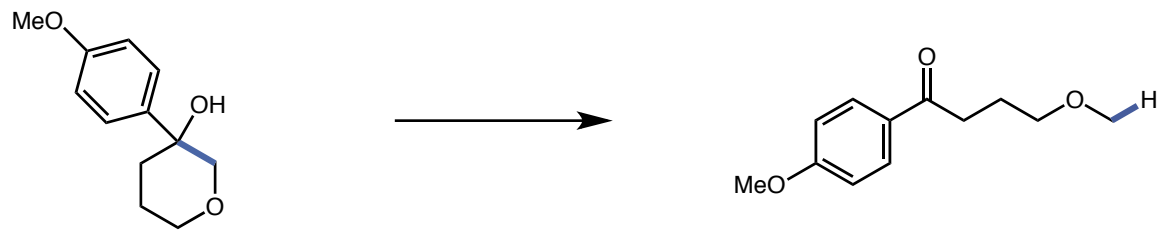

\section{4-methoxy-1-(4-methoxyphenyl)butan-1-one}

Prepared on $1 \mathrm{mmol}$ scale following the general procedure with 3-(4-methoxyphenyl)tetrahydro$2 \mathrm{H}$-pyran-3-ol. The crude material was first washed with $1.0 \mathrm{M} \mathrm{HCl}$ and then purified by alumina column chromatography to afford the titled compound as a brown solid (203 $\mathrm{mg}, 98 \%$ ). ${ }^{1}$ H NMR (500 MHz, C $\left.\mathbf{6}_{6} \mathbf{D}_{6}\right) \delta 7.88(\mathrm{~d}, J=8.8 \mathrm{~Hz}, 2 \mathrm{H}), 6.64(\mathrm{~d}, J=8.8 \mathrm{~Hz}, 2 \mathrm{H}), 3.24(\mathrm{t}, J=6.1$ $\mathrm{Hz}, 2 \mathrm{H}), 3.20(\mathrm{~s}, 3 \mathrm{H}), 3.08(\mathrm{~s}, 3 \mathrm{H}), 2.79(\mathrm{t}, J=7.2 \mathrm{~Hz}, 2 \mathrm{H}), 2.02(\mathrm{p}, J=6.6 \mathrm{~Hz}, 2 \mathrm{H}) .{ }^{13} \mathbf{C}$ NMR (126 MHz, $\left.\mathbf{C}_{6} \mathbf{D}_{6}\right) \delta 197.1,163.2,130.4,130.1,113.5,71.6,57.9,54.6,34.5,24.4$. IR (neat): 2929, 1677, 1601, 1577, 1510, 1257, 1171, 1117, 1030, $836 \mathrm{~cm}^{-1}$. HRMS (ESI): exact mass calculated for $[\mathrm{M}+\mathrm{H}]^{+}\left(\mathrm{C}_{12} \mathrm{H}_{17} \mathrm{O}_{3}\right)$ requires $m / z$ 209.11722, found $m / z 209.11708$.
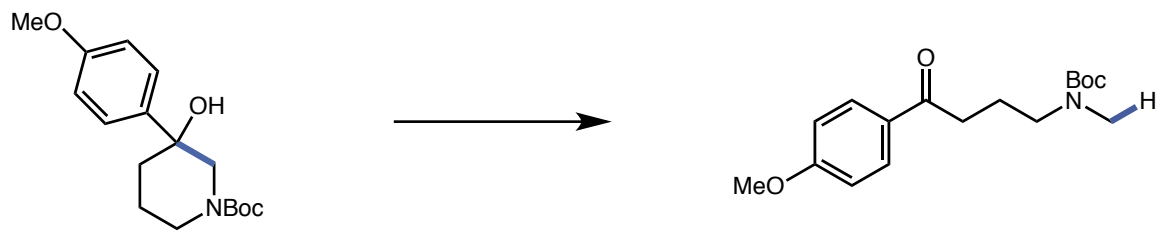

tert-butyl (4-(4-methoxyphenyl)-4-oxobutyl)(methyl)carbamate

Prepared on $1 \mathrm{mmol}$ scale following the general procedure with tert-butyl 3-hydroxy-3-(4methoxyphenyl)piperidine-1-carboxylate. The crude material was purified by silica gel column chromatography to afford the titled compound as a colorless oil (269 mg, 88\%). ${ }^{\mathbf{1}} \mathbf{H}$ NMR (500 MHz, $\left.\mathbf{C}_{6} \mathbf{D}_{6}, \mathbf{5 0}^{\circ} \mathbf{C}\right) \delta 7.87(\mathrm{~d}, J=8.9 \mathrm{~Hz}, 2 \mathrm{H}), 6.66(\mathrm{~d}, J=8.8 \mathrm{~Hz}, 2 \mathrm{H}), 3.24(\mathrm{~s}, 3 \mathrm{H}), 3.21-3.15$ $(\mathrm{m}, 2 \mathrm{H}), 2.67(\mathrm{~s}, 3 \mathrm{H}), 2.62(\mathrm{t}, J=6.5 \mathrm{~Hz}, 2 \mathrm{H}), 1.92-1.85(\mathrm{~m}, 2 \mathrm{H}), 1.42(\mathrm{~s}, 9 \mathrm{H}) .{ }^{13} \mathbf{C} \mathbf{N M R}(\mathbf{7 5}$ 
MHz, $\left.\mathbf{C}_{6} \mathbf{D}_{6}, \mathbf{5 0}^{\circ} \mathbf{C}\right) \delta 196.9,163.7,155.8,131.0,130.5,114.0,78.8,55.0,48.4,35.1,34.0,28.6$, 22.7. IR (neat): 2972, 1681, 1599, 1392, 1364, 1256, 1163, 1028, 983, 830, $770 \mathrm{~cm}^{-1}$. HRMS

(ESI): exact mass calculated for $[\mathrm{M}-\mathrm{Boc}+2 \mathrm{H}]^{+}\left(\mathrm{C}_{12} \mathrm{H}_{18} \mathrm{NO}_{2}\right)$ requires $m / z$ 208.13321, found 208.13327.

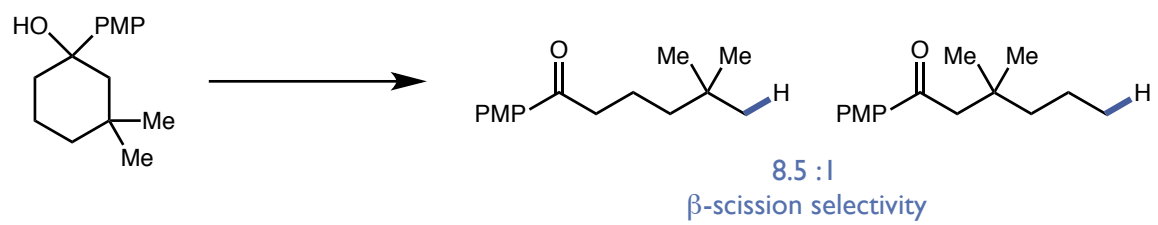

Prepared on $1 \mathrm{mmol}$ scale following the general procedure with 1-(4-methoxyphenyl)-3,3dimethylcyclohexan-1-ol. The crude material was purified by silica gel column chromatography to afford an 8.5:1 mixture of regioisomers as a pale yellow oil (220 mg, 94\%). The isomers were separated by preparative SFC on a Chiralpak AD-H column $(3 \times 25 \mathrm{~cm}$, conditions: $60 \mathrm{~mL} / \mathrm{min}$, $30 \%$ methanol, $220 \mathrm{~nm}, 10 \mathrm{mg} / \mathrm{mL}, 1 \mathrm{~mL} /$ injection) for final characterization.

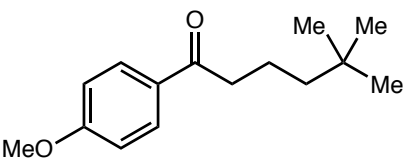

\section{1-(4-methoxyphenyl)-5,5-dimethylhexan-1-one (major isomer)}

${ }^{1} \mathbf{H}$ NMR $\left(500 \mathrm{MHz}, \mathbf{C}_{6} \mathbf{D}_{6}\right) \delta 7.91(\mathrm{~d}, J=8.9 \mathrm{~Hz}, 2 \mathrm{H}), 6.67(\mathrm{~d}, J=8.9 \mathrm{~Hz}, 2 \mathrm{H}), 3.20(\mathrm{~s}, 3 \mathrm{H})$, $2.57(\mathrm{t}, J=7.3 \mathrm{~Hz}, 2 \mathrm{H}), 1.85-1.62(\mathrm{~m}, 2 \mathrm{H}), 1.27-1.07(\mathrm{~m}, 2 \mathrm{H}), 0.88(\mathrm{~s}, 9 \mathrm{H}) .{ }^{13} \mathrm{C}$ NMR (126 MHz, $\left.\mathbf{C}_{\mathbf{6}} \mathbf{D}_{6}\right) \delta 197.1,163.1,130.7,130.1,113.5,54.5,43.8,38.7,30.1,29.1,19.5$. IR (neat): 3000, 2954, 2901, 2865, 1668, 1601, 1577, 1420, 1256, 1182, 1033, 978, 826, $819 \mathrm{~cm}^{-1}$. HRMS (ESI) exact mass calculated for $[\mathrm{M}+\mathrm{H}]^{+}\left(\mathrm{C}_{15} \mathrm{H}_{23} \mathrm{O}_{2}\right)$ requires $\mathrm{m} / \mathrm{z}$ 235.16926, found $\mathrm{m} / \mathrm{z}$ 235.16884 .

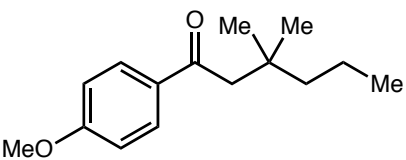

\section{1-(4-methoxyphenyl)-3,3-dimethylhexan-1-one (minor isomer)}

${ }^{1}$ H NMR (500 MHz, C6 $\left.\mathbf{D}_{6}\right) \delta 7.90(\mathrm{~d}, J=8.9 \mathrm{~Hz}, 2 \mathrm{H}), 6.65(\mathrm{~d}, J=8.9 \mathrm{~Hz}, 2 \mathrm{H}), 3.18(\mathrm{~s}, 3 \mathrm{H})$, $2.62(\mathrm{~s}, 2 \mathrm{H}), 1.43-1.35(\mathrm{~m}, 2 \mathrm{H}), 1.29-1.16(\mathrm{~m}, 2 \mathrm{H}), 1.06(\mathrm{~s}, 6 \mathrm{H}), 0.87(\mathrm{t}, J=7.2 \mathrm{~Hz}, 3 \mathrm{H}) .{ }^{13} \mathbf{C}$ NMR (126 MHz, $\left.\mathbf{C}_{6} \mathbf{D}_{6}\right) \delta 197.2,163.0,132.1,130.3,113.4,54.5,47.3,45.0,33.6,27.6,17.4$, 14.8. IR (neat): 2957, 2871, 1667, 1600, 1509, 1463, 1257, 1170, 1034, 1019, $844 \mathrm{~cm}^{-1}$. HRMS (ESI): exact mass calculated for $[\mathrm{M}+\mathrm{H}]^{+}\left(\mathrm{C}_{15} \mathrm{H}_{23} \mathrm{O}_{2}\right)$ requires $\mathrm{m} / \mathrm{z}$ 235.16926, found $\mathrm{m} / \mathrm{z}$ 235.16921 .
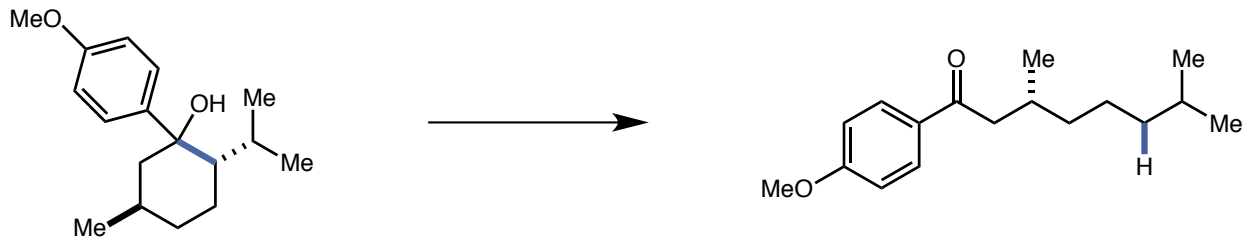

(R)-1-(4-methoxyphenyl)-3,7-dimethyloctan-1-one

Prepared on $1 \mathrm{mmol}$ scale following the general procedure with (2S,5R)-2-isopropyl-1-(4- 
methoxyphenyl)-5-methylcyclohexanol. The crude material was purified by silica gel column chromatography to afford the titled compound as a colorless oil $(235 \mathrm{mg}, 90 \%)$. ${ }^{\mathbf{1}} \mathbf{H}$ NMR (500 MHz, CDCl $) \delta 7.94(\mathrm{~d}, J=8.9 \mathrm{~Hz}, 2 \mathrm{H}), 6.93(\mathrm{~d}, J=8.9 \mathrm{~Hz}, 2 \mathrm{H}), 3.87(\mathrm{~s}, 3 \mathrm{H}), 2.89$ (dd, $J=$ 15.4, $5.7 \mathrm{~Hz}, 1 \mathrm{H}), 2.69(\mathrm{dd}, J=15.4,8.0 \mathrm{~Hz}, 1 \mathrm{H}), 2.21-2.08(\mathrm{~m}, 1 \mathrm{H}), 1.52(\mathrm{dp}, J=13.2,6.8$ $\mathrm{Hz}, 1 \mathrm{H}), 1.38-1.32(\mathrm{~m}, 2 \mathrm{H}), 1.31-1.25(\mathrm{~m}, 1 \mathrm{H}), 1.24-1.19(\mathrm{~m}, 1 \mathrm{H}), 1.19-1.10(\mathrm{~m}, 2 \mathrm{H})$, $0.94(\mathrm{~d}, J=6.7 \mathrm{~Hz}, 3 \mathrm{H}), 0.86(\mathrm{dd}, J=6.6,1.7 \mathrm{~Hz}, 6 \mathrm{H}) .{ }^{13} \mathbf{C}$ NMR $\left(\mathbf{1 2 6} \mathbf{~ M H z}, \mathbf{C D C l}_{3}\right) \delta 199.2$, 163.3, 130.6, 130.4, 113.6, 55.5, 45.7, 39.1, 37.5, 30.1, 28.0, 24.8, 22.7, 22.6, 20.1. IR (neat): 2954, 2926, 1673, 1599, 1576, 1509, 1257, 1168, 1031, 828, $808 \mathrm{~cm}-1$; HRMS (ESI): exact mass calculated for $[\mathrm{M}+\mathrm{H}]^{+}\left(\mathrm{C}_{17} \mathrm{H}_{27} \mathrm{O}_{2}\right)$ requires $\mathrm{m} / z$ 263.20056, found $\mathrm{m} / \mathrm{z} 263.20097$.

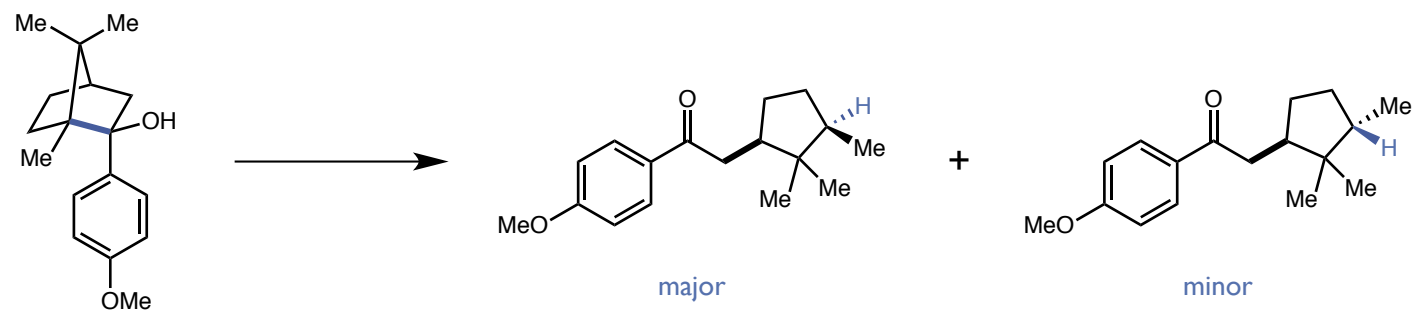

Prepared on $1 \mathrm{mmol}$ scale following the general procedure with $(1 R, 2 S, 4 R)-2-(4-$ methoxyphenyl)-1,7,7-trimethylbicyclo[2.2.1]heptan-2-ol. The crude material was purified by silica gel column chromatography to afford a 6:1 mixture of diastereomers as a colorless oil (238 $\mathrm{mg}, 91 \%)$. The diastereomers were separated via preparative SFC on a Chiralpak AD-H column $(2 \times 25 \mathrm{~cm}$, conditions: $70 \mathrm{~mL} / \mathrm{min}, 15 \%$ methanol, $220 \mathrm{~nm}, 7 \mathrm{mg} / \mathrm{mL}, 1 \mathrm{~mL} /$ injection) and their stereochemistries were determined by NOESY analysis (see product NMR section).<smiles>COc1ccc(C(=O)CC2CC[C@@H](C)C2(C)C)cc1</smiles>

1-(4-methoxyphenyl)-2-((1R,3R)-2,2,3-trimethylcyclopentyl)ethan-1-one (major isomer)

${ }^{1}$ H NMR (500 MHz, CDCl $)_{3} \delta 7.94(\mathrm{~d}, J=9.0 \mathrm{~Hz}, 2 \mathrm{H}), 6.93(\mathrm{~d}, J=8.9 \mathrm{~Hz}, 2 \mathrm{H}), 3.87(\mathrm{~s}, 3 \mathrm{H})$, $2.99(\mathrm{dd}, J=15.1,3.2 \mathrm{~Hz}, 1 \mathrm{H}), 2.66(\mathrm{dd}, J=15.1,10.8 \mathrm{~Hz}, 1 \mathrm{H}), 2.06-1.97(\mathrm{~m}, 1 \mathrm{H}), 1.89-$ $1.81(\mathrm{~m}, 1 \mathrm{H}), 1.79-1.71(\mathrm{~m}, 1 \mathrm{H}), 1.58-1.51(\mathrm{~m}, 1 \mathrm{H}), 1.27-1.11(\mathrm{~m}, 2 \mathrm{H}), 0.94(\mathrm{~s}, 3 \mathrm{H}), 0.85$ $(\mathrm{d}, J=6.9 \mathrm{~Hz}, 3 \mathrm{H}), 0.61$ (s, 3H). ${ }^{13} \mathbf{C}$ NMR (126 MHz, $\left.\mathbf{C D C l}_{3}\right) \delta$ 199.5, 163.3, 130.4, 127.3, 113.6, 55.5, 46.8, 44.6, 42.5, 39.6, 30.1, 28.3, 25.5, 14.8, 14.0. IR (neat): 2952, 2869, 1673, 1598, 1575, 1509, 1313, 1210, 1166, 1029, 991, 824, $807 \mathrm{~cm}^{-1}$. HRMS (ESI): exact mass calculated for $[\mathrm{M}+\mathrm{H}]^{+}\left(\mathrm{C}_{17} \mathrm{H}_{25} \mathrm{O}_{2}\right)$ requires $m / z$ 261.18491, found $m / z 261.18505$.<smiles>COc1ccc(C(=O)CC2CC[C@@H](C)C2(C)C)cc1</smiles>

1-(4-methoxyphenyl)-2-((1R,3S)-2,2,3-trimethylcyclopentyl)ethan-1-one (minor isomer)

${ }^{1}$ H NMR (500 MHz, CDCl $) \delta 7.94(\mathrm{~d}, J=8.9 \mathrm{~Hz}, 2 \mathrm{H}), 6.93(\mathrm{~d}, J=8.8 \mathrm{~Hz}, 2 \mathrm{H}), 3.87(\mathrm{~s}, 3 \mathrm{H})$, 2.97 (dd, $J=15.2,4.0 \mathrm{~Hz}, 1 \mathrm{H}), 2.68$ (dd, $J=15.3,10.5 \mathrm{~Hz}, 1 \mathrm{H}), 2.18$ (dtd, $J=10.2,7.9,3.9 \mathrm{~Hz}$, $1 \mathrm{H}), 1.93-1.87(\mathrm{~m}, 1 \mathrm{H}), 1.87-1.82(\mathrm{~m}, 1 \mathrm{H}), 1.66(\mathrm{~h}, J=7.0 \mathrm{~Hz}, 1 \mathrm{H}), 1.27-1.13(\mathrm{~m}, 2 \mathrm{H})$, 0.89 (s, 3H), $0.87(\mathrm{~s}, 3 \mathrm{H}), 0.86(\mathrm{~d}, 3 \mathrm{H}) .{ }^{13} \mathbf{C}$ NMR (126 MHz, CDCl $\left.\mathbf{3}\right) \delta 199.6,163.2,130.5$, 130.4, 113.7, 55.5, 44.4, 43.5, 42.3, 40.1, 31.4, 29.5, 24.3, 23.6, 16.4. IR (neat): 2952, 2869, 1673, 1598, 1575, 1509, 1313, 1210, 1166, 1029, 991, 824, $807 \mathrm{~cm}^{-1}$. HRMS (ESI): exact mass calculated for $[\mathrm{M}+\mathrm{H}]^{+}\left(\mathrm{C}_{17} \mathrm{H}_{25} \mathrm{O}_{2}\right)$ requires $m / z$ 261.18491, found $m / z$ 261.18505. 

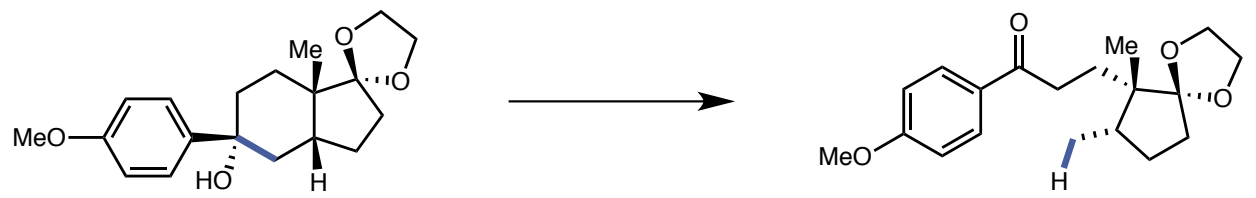

3-((6S,7S)-6,7-dimethyl-1,4-dioxaspiro[4.4]nonan-6-yl)-1-(4-methoxyphenyl)-propan-1-one Prepared on $1 \mathrm{mmol}$ scale following the general procedure with $\left(3 \mathrm{a}^{\prime} R, 7 \mathrm{a}^{\prime} S\right)-5^{\prime}-(4-$ methoxyphenyl)-7a'-methyloctahydrospiro[[1,3]dioxolane-2,1'-inden]-5'-ol. The crude material was purified by silica gel column chromatography to afford the titled compound as a colorless oil $(195 \mathrm{mg}, 61 \%) .{ }^{1} \mathbf{H}$ NMR (500 MHz, $\left.\mathbf{C}_{6} \mathbf{D}_{\mathbf{6}}\right) \delta 8.09(\mathrm{~d}, J=8.8 \mathrm{~Hz}, 2 \mathrm{H}), 6.68(\mathrm{~d}, J=8.9 \mathrm{~Hz}, 2 \mathrm{H})$, $3.50-3.39$ (m, 4H), 3.34 (ddd, $J=16.2,11.1,5.1 \mathrm{~Hz}, 1 \mathrm{H}), 3.18$ (s, 3H), 2.88 (ddd, $J=16.2$, 11.0, 5.2 Hz, 1H), $2.08-2.02(\mathrm{~m}, 1 \mathrm{H}), 2.02-1.94(\mathrm{~m}, 2 \mathrm{H}), 1.89-1.84(\mathrm{~m}, 1 \mathrm{H}), 1.82-1.74(\mathrm{~m}$, 2H), $1.28-1.20(\mathrm{~m}, 1 \mathrm{H}), 1.05(\mathrm{~s}, 3 \mathrm{H}), 0.96(\mathrm{~d}, J=7.1 \mathrm{~Hz}, 3 \mathrm{H}) \cdot{ }^{13} \mathbf{C} \mathbf{N M R}\left(\mathbf{1 2 6} \mathbf{~ M H z}, \mathbf{C}_{6} \mathbf{D}_{\mathbf{6}}\right) \delta$ 198.3, 163.0, 130.8, 130.3, 120.1, 113.6, 64.7, 63.3, 54.5, 47.0, 41.8, 34.0, 32.8, 27.4, 25.8, 18.9, 15.5. IR (neat): 2958, 2876, 1673, 1599, 1575, 1509, 1306, 1255, 1169, 1028, 986, $839 \mathrm{~cm}^{-1}$. HRMS (ESI): exact mass calculated for $[\mathrm{M}+\mathrm{H}]^{+}\left(\mathrm{C}_{19} \mathrm{H}_{27} \mathrm{O}_{4}\right)$ requires $\mathrm{m} / \mathrm{z} 319.19039$, found $\mathrm{m} / \mathrm{z}$ 319.19003 .<smiles>COc1ccc(C2CCCC[C@@]2(N)O)cc1</smiles><smiles>COc1ccc(CCCCC(C)=O)cc1</smiles>

\section{7-(4-methoxyphenyl)heptan-2-one}

Prepared on $1 \mathrm{mmol}$ scale following the general procedure with 2-(4-methoxyphenyl)-1methylcyclohexanol. The crude material was purified by silica gel column chromatography to afford the titled compound as a colorless oil (200 mg, 91\%). ${ }^{1} \mathbf{H}$ NMR (500 $\left.\mathbf{~ M H z}, \mathbf{C D C l}_{3}\right) \delta 7.08$ $(\mathrm{d}, J=8.6 \mathrm{~Hz}, 2 \mathrm{H}), 6.82(\mathrm{~d}, J=8.6 \mathrm{~Hz}, 2 \mathrm{H}), 3.78(\mathrm{~s}, 3 \mathrm{H}), 2.58-2.49(\mathrm{~m}, 2 \mathrm{H}), 2.41(\mathrm{t}, J=7.5$ $\mathrm{Hz}, 2 \mathrm{H}), 2.12$ (s, 3H), $1.64-1.54(\mathrm{~m}, 4 \mathrm{H}), 1.35-1.27(\mathrm{~m}, 2 \mathrm{H}) .{ }^{13} \mathbf{C}$ NMR (126 MHz, $\left.\mathbf{C D C l}_{3}\right) \delta$ 209.3, 157.6, 134.6, 129.2, 113.7, 55.3, 43.7, 34.8, 31.5, 29.9, 28.7, 23.7. IR (neat): 2930, 2855, $1712,1611,1510,1242,1175,1034,822 \mathrm{~cm}^{-1}$. HRMS (EI): exact mass calculated for $[\mathrm{M} \bullet]^{+}$ $\left(\mathrm{C}_{14} \mathrm{H}_{20} \mathrm{O}_{2}\right)$ requires $m / z 220.1458$, found $m / z 220.1454$.

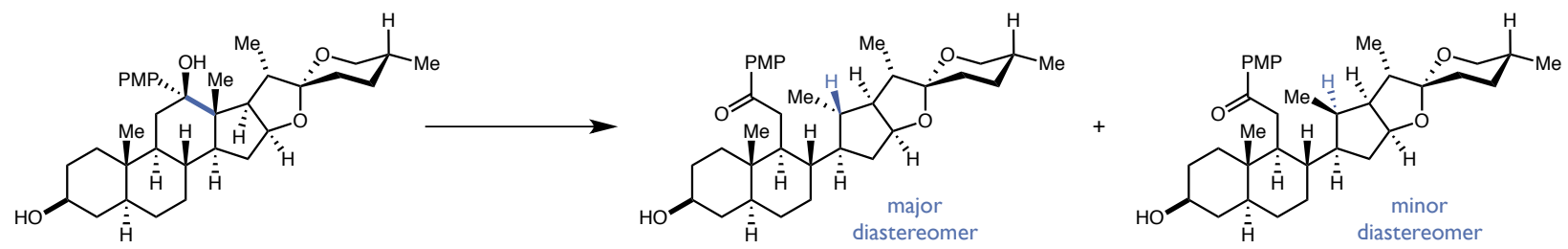

Prepared on $0.5 \mathrm{mmol}$ scale following the general procedure with (2a $\left.S, 4 S, 5^{\prime} R, 6 \mathrm{a} S, 6 \mathrm{~b} S, 8 R, 8 \mathrm{~b} R, 9 S, 10 R, 11 \mathrm{a} S, 12 \mathrm{a} S, 12 \mathrm{~b} R\right)-8$-(4-methoxyphenyl)-5',6a, 8a,9tetramethyldocosahydrospiro[naphtho[2',1':4,5]indeno[2,1-b]furan-10,2'-pyran]-4,8-diol $\mathrm{mg}$ ). The crude material was purified by silica gel column chromatography to afford an $8: 1$ mixture of diastereomers as a colorless oil $(220 \mathrm{mg}, 81 \%)$. The diastereomer were separated via preparative SFC on a Chiralpak AD-H column $(3 \times 25 \mathrm{~cm}$, conditions: $60 \mathrm{~mL} / \mathrm{min}, 30 \%$ isopropanol, $220 \mathrm{~nm}, 7 \mathrm{mg} / \mathrm{mL}, 1 \mathrm{~mL} /$ injection) and their stereochemistries were determined by NOESY analysis (see product NMR section). 


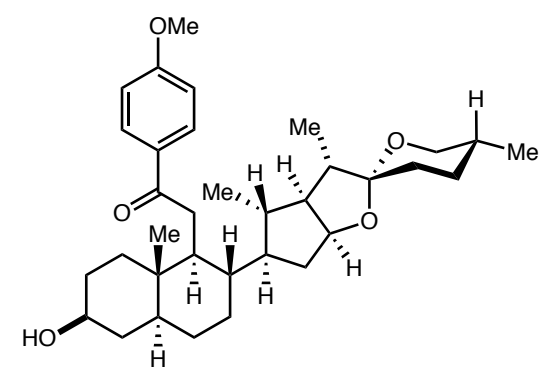

2-((1S,2S,4aS,6S,8aS)-6-hydroxy-8a-methyl-2-((2R,3S,3a $\left.S, 4 S, 5 R, 5^{\prime} R, 6 \mathrm{a} S\right)-3,4,5^{\prime}-$ trimethyldecahydrospiro[cyclopenta[b]furan-2,2'-pyran]-5-yl)decahydronaphthalen-1-yl)1-(4-methoxyphenyl)ethan-1-one (major isomer)

${ }^{1} \mathbf{H}$ NMR $\left(500 \mathrm{MHz}, \mathbf{C}_{\mathbf{6}} \mathbf{D}_{\mathbf{6}}\right) \delta 7.96(\mathrm{~d}, J=8.9 \mathrm{~Hz}, 2 \mathrm{H}), 6.67(\mathrm{~d}, J=8.8 \mathrm{~Hz}, 2 \mathrm{H}), 4.39(\mathrm{dt}, J=8.8$, $7.1 \mathrm{~Hz}, 1 \mathrm{H}), 3.57-3.41(\mathrm{~m}, 2 \mathrm{H}), 3.34(\mathrm{tt}, J=10.7,4.9 \mathrm{~Hz}, 1 \mathrm{H}), 3.16(\mathrm{~s}, 3 \mathrm{H}), 2.74(\mathrm{dd}, J=18.1$, $5.9 \mathrm{~Hz}, 1 \mathrm{H}), 2.60(\mathrm{dd}, J=18.0,3.0 \mathrm{~Hz}, 1 \mathrm{H}), 2.38(\mathrm{ddd}, J=12.5,7.1,5.5 \mathrm{~Hz}, 1 \mathrm{H}), 2.16(\mathrm{ddd}, J=$ $11.3,5.9,3.0 \mathrm{~Hz}, 1 \mathrm{H}), 1.75-1.65(\mathrm{~m}, 3 \mathrm{H}), 1.65-1.39(\mathrm{~m}, 11 \mathrm{H}), 1.39-1.31(\mathrm{~m}, 1 \mathrm{H}), 1.31-$ $1.24(\mathrm{~m}, 2 \mathrm{H}), 1.24-1.14(\mathrm{~m}, 4 \mathrm{H}), 1.11(\mathrm{~d}, J=6.8 \mathrm{~Hz}, 3 \mathrm{H}), 1.00-0.89(\mathrm{~m}, 2 \mathrm{H}), 0.87(\mathrm{~d}, J=6.5$ $\mathrm{Hz}, 3 \mathrm{H}), 0.69(\mathrm{~s}, 3 \mathrm{H}), 0.64(\mathrm{~d}, J=6.3 \mathrm{~Hz}, 3 \mathrm{H}) .{ }^{13} \mathbf{C}$ NMR (126 MHz, $\left.\mathbf{C}_{6} \mathbf{D}_{6}\right) \delta$ 196.3, 163.2, 130.6, 130.0, 113.7, 108.3, 80.4, 70.3, 66.6, 57.6, 54.5, 50.4, 50.1, 45.3, 43.8, 42.6, 38.4, 37.7, $37.1,36.8,36.6,32.7,31.9,31.4,30.5,28.9,28.4,25.6,17.9,17.0,13.7,12.1$. IR (neat): 3405, 2925, 2862, 1676, 1599, 1575, 1510, 1456, 1255, 1169, 1032, 1052, 978, 828, 812, $755 \mathrm{~cm}^{-1}$. HRMS (ESI): exact mass calculated for $[\mathrm{M}+\mathrm{H}]^{+}\left(\mathrm{C}_{34} \mathrm{H}_{51} \mathrm{O}_{5}\right)$ requires $\mathrm{m} / z$ 539.37310, found $\mathrm{m} / \mathrm{z}$ 539.37341 .

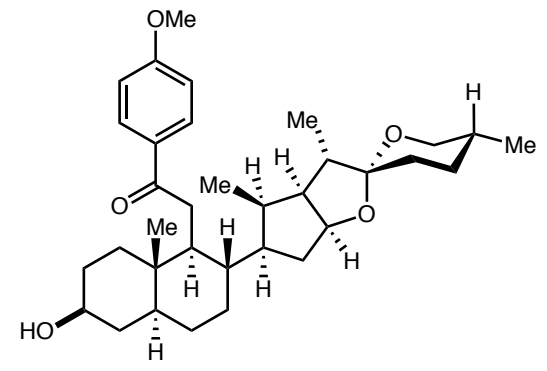

2-((1S,2S,4aS,6S,8a $S)-6-h y d r o x y-8 \mathrm{a}-\mathrm{methyl}^{-2}-\left(\left(2 R, 3 S, 3 \mathrm{a} S, 4 R, 5 R, 5^{\prime} R, 6 \mathrm{a} S\right)-3,4,5^{\prime}-\right.$ trimethyldecahydrospiro[cyclopenta[b]furan-2,2'-pyran]-5-yl)decahydronaphthalen-1-yl)1-(4-methoxyphenyl)ethan-1-one (minor isomer)

${ }^{1}$ H NMR (800 MHz, C $\left.\mathbf{C}_{\mathbf{6}} \mathbf{D}_{\mathbf{6}}\right) \delta 7.97(\mathrm{~d}, J=8.8 \mathrm{~Hz}, 2 \mathrm{H}), 6.67(\mathrm{~d}, J=8.8 \mathrm{~Hz}, 2 \mathrm{H}), 4.43-4.37$ (m, $1 \mathrm{H}), 3.51-3.44(\mathrm{~m}, 2 \mathrm{H}), 3.41-3.36(\mathrm{~m}, 1 \mathrm{H}), 3.18(\mathrm{~s}, 3 \mathrm{H}), 2.79(\mathrm{dd}, J=18.5,2.8 \mathrm{~Hz}, 1 \mathrm{H}), 2.71$ $(\mathrm{dd}, J=18.6,5.7 \mathrm{~Hz}, 1 \mathrm{H}), 2.26-2.20(\mathrm{~m}, 2 \mathrm{H}), 2.17(\mathrm{dq}, J=11.6,5.7 \mathrm{~Hz}, 1 \mathrm{H}), 1.88(\mathrm{~h}, J=7.5$ $\mathrm{Hz}, 1 \mathrm{H}), 1.84-1.76(\mathrm{~m}, 2 \mathrm{H}), 1.71-1.60(\mathrm{~m}, 6 \mathrm{H}), 1.57-1.43(\mathrm{~m}, 4 \mathrm{H}), 1.34-1.26(\mathrm{~m}, 2 \mathrm{H})$, $1.24-1.11(\mathrm{~m}, 5 \mathrm{H}), 1.04(\mathrm{td}, J=14.2,13.7,3.8 \mathrm{~Hz}, 1 \mathrm{H}), 1.01-0.95(\mathrm{~m}, 4 \mathrm{H}), 0.83(\mathrm{dd}, J=7.4$, $2.2 \mathrm{~Hz}, 3 \mathrm{H}), 0.72(\mathrm{~s}, 3 \mathrm{H}), 0.63(\mathrm{~d}, J=6.3 \mathrm{~Hz}, 3 \mathrm{H}) .{ }^{13} \mathbf{C}$ NMR (126 MHz, C6 $\left.\mathbf{D}_{6}\right) \delta$ 197.2, 163.6, $130.9,130.3,114.1,108.9,81.0,70.8,67.1,54.9,53.2,47.2,46.4,44.2,43.2,38.7,38.6,38.3$, $37.6,37.5,37.4,31.9,31.71,31.69,31.5,30.8,29.3,29.0,17.4,14.2,12.9,12.6$. IR (neat): 3413, 2926, 2860, 1677, 1599, 1575, 1455, 1258, 1243, 1169, 1057, 978, 899, $812 \mathrm{~cm}^{-1}$. HRMS (ESI): exact mass calculated for $[\mathrm{M}+\mathrm{H}]^{+}\left(\mathrm{C}_{34} \mathrm{H}_{51} \mathrm{O}_{5}\right)$ requires $\mathrm{m} / z$ 539.37310, found $\mathrm{m} / \mathrm{z}$ 539.37331 . 

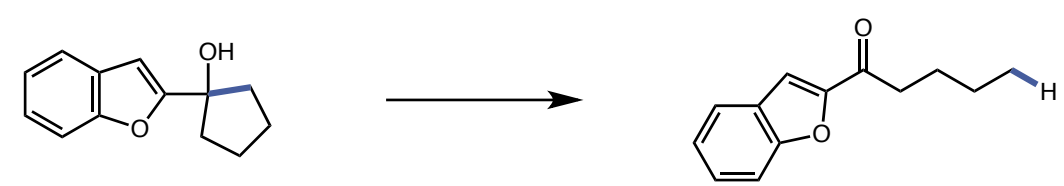

\section{1-(benzofuran-2-yl)pentan-1-one}

Prepared on $1 \mathrm{mmol}$ scale following the general procedure with 1-(benzofuran-2yl)cyclopentanol. The crude material was purified by silica gel column chromatography to afford the titled compound as a white solid (169 mg, 84\%). ${ }^{1} \mathbf{H}$ NMR $\left(\mathbf{5 0 0} \mathbf{~ M H z}, \mathbf{C}_{6} \mathbf{D}_{\mathbf{6}}\right) \delta 7.28-7.26$ $(\mathrm{m}, 2 \mathrm{H}), 7.08-7.04(\mathrm{~m}, 2 \mathrm{H}), 6.99-6.96(\mathrm{~m}, 1 \mathrm{H}), 2.61(\mathrm{t}, J=7.3 \mathrm{~Hz}, 2 \mathrm{H}), 1.65(\mathrm{p}, J=7.4 \mathrm{~Hz}$, 2H), 1.23 (h, $J=7.4 \mathrm{~Hz}, 2 \mathrm{H}), 0.81(\mathrm{t}, J=7.4 \mathrm{~Hz}, 3 \mathrm{H}) .{ }^{13} \mathbf{C}$ NMR (126 MHz, C6 $\left.\mathbf{C}_{6}\right) \delta 190.1$, $155.4,153.3,128.2,127.3,123.5,123.0,112.1,111.2,38.4,25.8,22.3,13.7$. IR (neat): 2959, 2932, 2872, 1682, 1556, 1280, 1159, 1141, 1027, $755 \mathrm{~cm}^{-1}$. HRMS (ESI): exact mass calculated for $[\mathrm{M}+\mathrm{H}]^{+}\left(\mathrm{C}_{13} \mathrm{H}_{15} \mathrm{O}_{2}\right)$ requires $m / z$ 203.10666, found $\mathrm{m} / z 203.10638$.
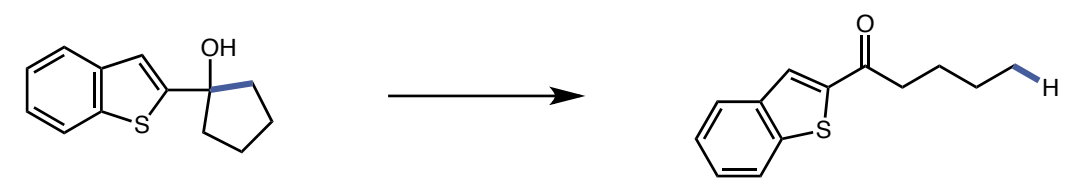

\section{1-(benzo[b]thiophen-2-yl)pentan-1-one}

Prepared on $1 \mathrm{mmol}$ scale following the general procedure with 1-(benzo[b]thiophen-2yl)cyclopentanol. The crude material was purified by silica gel column chromatography to afford the titled compound as an off-white powder $(188 \mathrm{mg}, 86 \%) .{ }^{1} \mathbf{H} \mathbf{N M R}\left(\mathbf{5 0 0} \mathbf{~ M H z}, \mathbf{C}_{6} \mathbf{D}_{\mathbf{6}}\right) \delta 7.51$ $(\mathrm{d}, J=8.0 \mathrm{~Hz}, 1 \mathrm{H}), 7.37-7.35(\mathrm{~m}, 2 \mathrm{H}), 7.10-7.07(\mathrm{~m}, 1 \mathrm{H}), 7.03-7.00(\mathrm{~m}, 1 \mathrm{H}), 2.52(\mathrm{t}, J=$ $7.3 \mathrm{~Hz}, 2 \mathrm{H}), 1.64(\mathrm{p}, J=7.4 \mathrm{~Hz}, 2 \mathrm{H}), 1.23(\mathrm{~h}, J=7.4 \mathrm{~Hz}, 2 \mathrm{H}), 0.82(\mathrm{t}, J=7.4 \mathrm{~Hz}, 3 \mathrm{H}) .{ }^{13} \mathbf{C}$ NMR (126 MHz, $\left.\mathbf{C}_{\mathbf{6}} \mathbf{D}_{\mathbf{6}}\right) \delta 193.3,144.5,142.4,139.3,128.1,126.9,125.5,124.6,122.9,38.5$, 26.4, 22.3, 13.7. IR (neat): 2961, 2951, 2929, 2871, 1658, 1519, 1407, 1177, 744, $727 \mathrm{~cm}^{-1}$. HRMS (ESI): exact mass calculated for $[\mathrm{M}+\mathrm{H}]^{+}\left(\mathrm{C}_{13} \mathrm{H}_{15} \mathrm{OS}\right)$ requires $\mathrm{m} / \mathrm{z} 219.08381$, found $\mathrm{m} / \mathrm{z}$ 219.08372 .
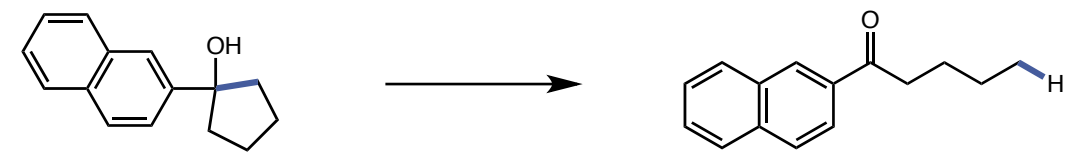

\section{1-(naphthalen-2-yl)pentan-1-one}

Prepared on $1 \mathrm{mmol}$ scale following the general procedure with 1-(naphthalen-2yl)cyclopentanol. The crude material was purified by silica gel column chromatography to afford the titled compound as a yellow solid $(88 \mathrm{mg}, 41 \%)$. Spectra are consistent with reported literature values. ${ }^{10}$
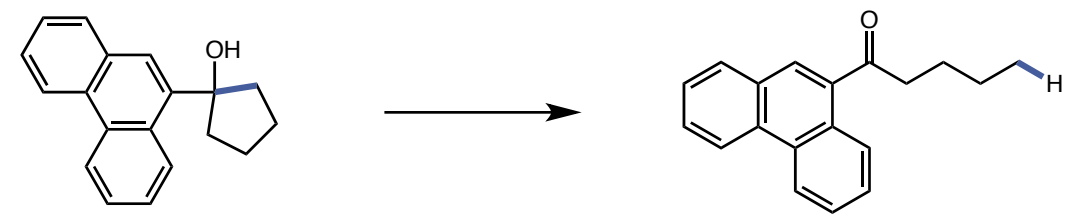

\section{1-(phenanthren-9-yl)pentan-1-one}

Prepared on $1 \mathrm{mmol}$ scale following the general procedure with 1-(phenanthren-9yl)cyclopentanol. The crude material was purified by silica gel column chromatography to afford the titled compound as a yellow solid $(215 \mathrm{mg}, 82 \%) .{ }^{\mathbf{1}} \mathbf{H} \mathbf{~ N M R}\left(\mathbf{5 0 0} \mathbf{~ M H z}, \mathbf{C}_{\mathbf{6}} \mathbf{D}_{\mathbf{6}}\right) \delta 8.92(\mathrm{~d}, J=$ 
$8.2 \mathrm{~Hz}, 1 \mathrm{H}), 8.43(\mathrm{~d}, J=8.2 \mathrm{~Hz}, 1 \mathrm{H}), 8.36(\mathrm{~d}, J=8.3 \mathrm{~Hz}, 1 \mathrm{H}), 7.73(\mathrm{~s}, 1 \mathrm{H}), 7.58(\mathrm{~d}, J=7.8 \mathrm{~Hz}$, 1H), $7.45-7.32(\mathrm{~m}, 4 \mathrm{H}), 2.71(\mathrm{t}, J=7.3 \mathrm{~Hz}, 2 \mathrm{H}), 1.73$ (p, $J=7.5 \mathrm{~Hz}, 2 \mathrm{H}), 1.29$ (h, $J=7.4 \mathrm{~Hz}$, 2H), 0.85 (t, $J=7.4 \mathrm{~Hz}, 3 \mathrm{H}) .{ }^{13} \mathbf{C}$ NMR (126 MHz, $\left.\mathbf{C}_{6} \mathbf{D}_{6}\right) \delta 203.2,135.9,131.8,130.9,130.2$, $129.5,128.9,128.7,128.2,127.4,127.0,126.9,126.7,122.8,122.7,41.6,26.6,22.4,13.8$. IR (neat): 2957, 2929, 2871, 1678, 1448, 1249, 1130, 1085, 747, $725 \mathrm{~cm}^{-1}$. HRMS (ESI): exact mass calculated for $[\mathrm{M}+\mathrm{H}]^{+}\left(\mathrm{C}_{19} \mathrm{H}_{19} \mathrm{O}\right)$ requires $m / z$ 263.14304, found $m / z 263.14316$.

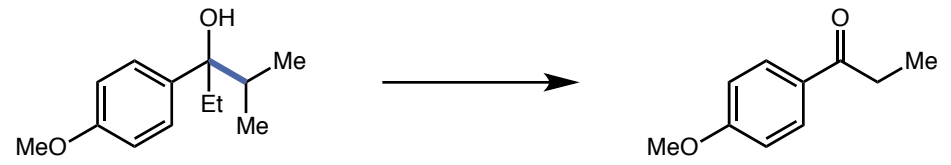

1-(4-methoxyphenyl)propan-1-one

Prepared on $1 \mathrm{mmol}$ scale following the general procedure with 3-(4-methoxyphenyl)-2methylpentan-3-ol. The crude material was purified by silica gel column chromatography to afford the titled compound as a yellow oil (152 mg, 93\%). Spectra are consistent with reported literature values. ${ }^{11}$
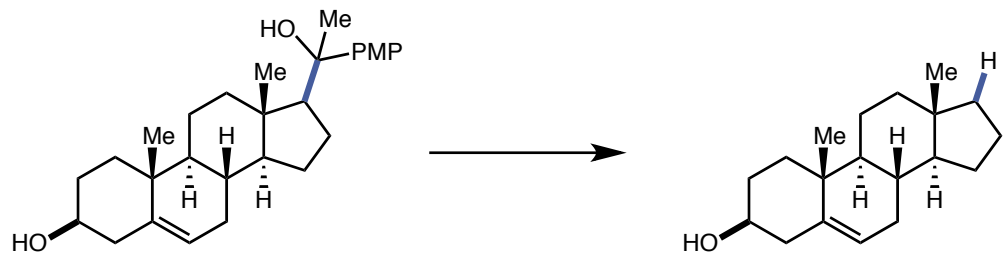

$(3 S, 8 S, 9 S, 10 R, 13 S, 14 S)-10,13-d i m e t h y l-2,3,4,7,8,9,10,11,12,13,14,15,16,17$-tetradecahydro$1 H$-cyclopenta $[a]$ phenanthren-3-ol

Prepared on $0.5 \mathrm{mmol}$ scale following the general procedure from $(3 S, 9 S, 10 R, 13 S, 14 S, 17 S)-17$ (1-hydroxy-1-(4-methoxyphenyl)ethyl)-10,13-dimethyl-2,3,4,7,8,9,10,11,12,13,14,15,16,17-

tetradecahydro- $1 H$-cyclopenta $[a]$ phenanthren-3-ol. The product was purified by silica gel column chromatography to afford the titled compound as a colorless oil $(98 \mathrm{mg}, 72 \%)$. ${ }^{\mathbf{1}} \mathbf{H}$ NMR $\left(\mathbf{5 0 0} \mathbf{M H z}, \mathbf{C}_{\mathbf{6}} \mathbf{D}_{\mathbf{6}}\right) \delta 5.34(\mathrm{dt}, J=4.8,2.1 \mathrm{~Hz}, 1 \mathrm{H}), 3.39$ (tt, $\left.J=11.1,4.8 \mathrm{~Hz}, 1 \mathrm{H}\right), 2.24$ (dtdd, $J=$ 16.0, 13.3, 5.3, $2.3 \mathrm{~Hz}, 2 \mathrm{H}), 1.95$ (dtd, $J=17.5,5.3,2.6 \mathrm{~Hz}, 1 \mathrm{H}), 1.76-1.70(\mathrm{~m}, 1 \mathrm{H}), 1.69-$ $1.49(\mathrm{~m}, 6 \mathrm{H}), 1.48-1.30(\mathrm{~m}, 5 \mathrm{H}), 1.12$ (dddd, $J=17.2,11.9,7.6,4.3 \mathrm{~Hz}, 3 \mathrm{H}), 1.00-0.86(\mathrm{~m}$, 3H), $0.92(\mathrm{~s}, 3 \mathrm{H}), 0.84-0.74(\mathrm{~m}, 1 \mathrm{H}), 0.68(\mathrm{~s}, 3 \mathrm{H}) .{ }^{13} \mathbf{C}$ NMR (126 MHz, $\left.\mathbf{C}_{6} \mathbf{D}_{6}\right) \delta 140.9,121.3$, 71.3, 54.7, 50.4, 42.6, 40.5, 40.3, 38.7, 37.4, 36.6, 32.2, 32.1, 31.8, 25.6, 21.2, 20.6, 19.2, 17.1 . IR (neat): 3242, 2930, 2858, 1610, 1453, 1376, 1232, 1110, 1019, 954, 812, 795, 739, $677 \mathrm{~cm}^{-1}$. HRMS (ESI): exact mass calculated for $\left[\mathrm{M}-\mathrm{H}_{2} \mathrm{O}+\mathrm{H}\right]^{+}\left(\mathrm{C}_{19} \mathrm{H}_{29}\right)$ requires $m / z 257.22638$, found $m / z 257.22619$.
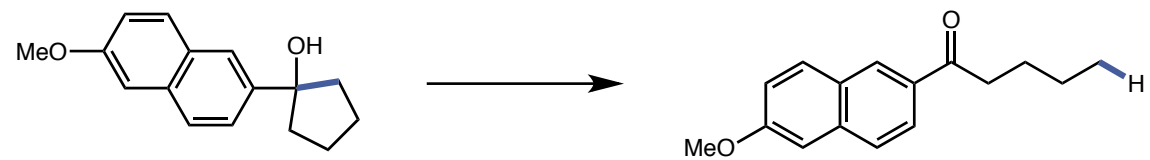

\section{1-(6-methoxynaphthalen-2-yl)pentan-1-one}

Prepared on $1 \mathrm{mmol}$ scale in a single run, following the general procedure with 1-(6methoxynaphthalen-2-yl)cyclopentanol. The crude material was purified by silica gel column chromatography to afford the titled compound as a yellow solid (18 mg, 7\%). ${ }^{\mathbf{1}} \mathbf{H}$ NMR (500 MHz, $\left.\mathbf{C}_{6} \mathbf{D}_{6}\right) \delta 8.27(\mathrm{~s}, 1 \mathrm{H}), 8.20(\mathrm{~d}, J=8.6 \mathrm{~Hz}, 1 \mathrm{H}), 7.51(\mathrm{dd}, J=19.3,8.7 \mathrm{~Hz}, 2 \mathrm{H}), 7.12(\mathrm{~d}, J=$ $2.5 \mathrm{~Hz}, 1 \mathrm{H}), 6.83(\mathrm{~d}, J=2.6 \mathrm{~Hz}, 1 \mathrm{H}), 3.33(\mathrm{~s}, 3 \mathrm{H}), 2.72(\mathrm{t}, J=7.3 \mathrm{~Hz}, 2 \mathrm{H}), 1.76(\mathrm{p}, J=7.4 \mathrm{~Hz}$, 2H), 1.31 (h, $J=7.4 \mathrm{~Hz}, 2 \mathrm{H}), 0.88$ (t, $J=7.4 \mathrm{~Hz}, 3 \mathrm{H}) .{ }^{13} \mathbf{C}$ NMR (126 MHz, $\left.\mathbf{C}_{6} \mathbf{D}_{6}\right) \delta 198.2$, 
159.6, 137.2, 133.0, 131.0, 129.4, 128.1, 127.0, 124.9, 119.6, 105.7, 54.5, 37.8, 26.5, 22.5, 13.9. IR (neat): 2955, 2936, 2871, 1675, 1623, 1603, 1482, 1266, 1164, 1030, 852, $811 \mathrm{~cm}^{-1}$. HRMS (ESI): exact mass calculated for $[\mathrm{M}+\mathrm{H}]^{+}\left(\mathrm{C}_{16} \mathrm{H}_{19} \mathrm{O}_{2}\right)$ requires $\mathrm{m} / \mathrm{z}$ 243.13796, found $\mathrm{m} / \mathrm{z}$ 243.13762 . 


\section{Protocol for forming distally fluorinated ketones via PCET-mediated ring-opening of cyclic alcohols}
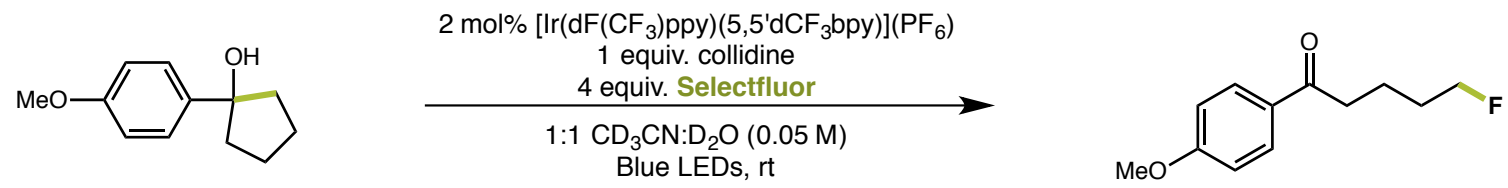

\section{5-fluoro-1-(4-methoxyphenyl)pentan-1-one}

A screw cap culture tube $(16 \times 125 \mathrm{~mm})$ outfitted with a PTFE/silicone septa was charged with 1-(4-methoxyphenyl)cyclopentan-1-ol $(0.5 \mathrm{mmol}, 1$ equiv, $96 \mathrm{mg})$, redistilled collidine $(0.5$

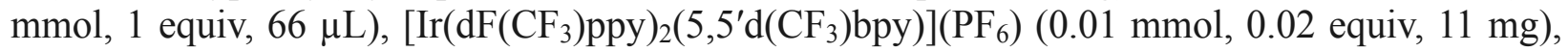
and Selectfluor ( $2 \mathrm{mmol}, 4$ equiv, $709 \mathrm{mg}$ ). The vial was then evacuated and backfilled with nitrogen three times. Degassed acetonitrile- $d_{3}(5 \mathrm{~mL})$ and water- $d_{2}(5 \mathrm{~mL})$ were added to form a suspension. The mixture was sparged with nitrogen for 20 minutes. The reaction was irradiated with blue LEDs strips set inside a beaker, and let stir at room temperature with a fan to cool the reaction setup. After 3 hour, the reaction was concentrated, washed with water, extracted with EtOAc, then purified by silica gel column chromatography to afford the titled compound as a white solid (55 mg, 52\%). ${ }^{1} \mathbf{H}$ NMR (500 MHz, $\left.\mathbf{C}_{6} \mathbf{D}_{6}\right) \delta 7.84(\mathrm{~d}, J=8.9 \mathrm{~Hz}, 2 \mathrm{H}), 6.66(\mathrm{~d}, J=$ $8.9 \mathrm{~Hz}, 2 \mathrm{H}), 4.14(\mathrm{t}, J=6.0 \mathrm{~Hz}, 1 \mathrm{H}), 4.05(\mathrm{t}, J=6.0 \mathrm{~Hz}, 1 \mathrm{H}), 3.20$ (s, 3H), 2.49 (t, $J=7.2 \mathrm{~Hz}$, 2H), 1.71 (dt, $J=15.00,7.3 \mathrm{~Hz}, 2 \mathrm{H}), 1.50-1.39(\mathrm{~m}, 2 \mathrm{H}) .{ }^{13} \mathbf{C}$ NMR (126 MHz, $\left.\mathbf{C}_{6} \mathbf{D}_{6}\right) \delta 197.0$, 163.6, 130.8, 130.5, 113.9, 83.7 (d, $J=166 \mathrm{~Hz}), 54.9,37.5,30.1,20.3$. IR (neat): 2940, 1675, 1599, 1576, 1510, 1256, 1207, 1169, 1029, $815 \mathrm{~cm}^{-1}$. HRMS (ESI): exact mass calculated for $[\mathrm{M}+\mathrm{H}]^{+}\left(\mathrm{C}_{12} \mathrm{H}_{16} \mathrm{FO}_{2}\right)$ requires $m / z 211.11288$, found $\mathrm{m} / z$ 211.11271.

\section{Protocol for forming distally chlorinated ketones via PCET-mediated ring-opening of cyclic alcohols}
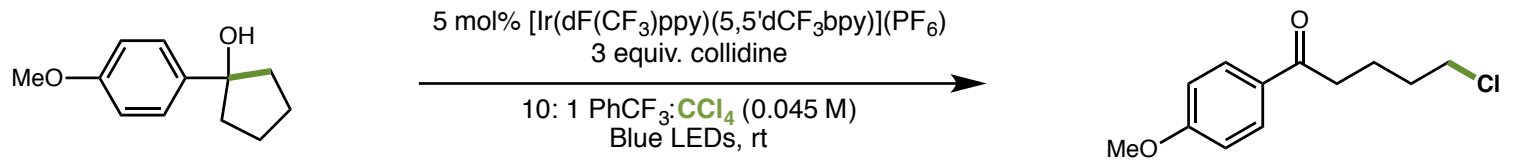

\section{5-chloro-1-(4-methoxyphenyl)pentan-1-one}

A screw cap culture tube $(16 \times 125 \mathrm{~mm})$ outfitted with a PTFE/silicone septa was charged with 1-(4-methoxyphenyl)cyclopentan-1-ol $(0.5 \mathrm{mmol}, 1.0$ equiv, $96 \mathrm{mg})$, redistilled collidine (1.5 mmol, 3 equiv, $198 \mu \mathrm{L})$, and $\left[\operatorname{Ir}\left(\mathrm{dF}\left(\mathrm{CF}_{3}\right) \text { ppy }\right)_{2}\left(5,5^{\prime} \mathrm{d}\left(\mathrm{CF}_{3}\right)\right.\right.$ bpy $\left.)\right]\left(\mathrm{PF}_{6}\right)(0.025 \mathrm{mmol}, 0.05$ equiv, 26 $\mathrm{mg})$ in dry trifluorotoluene $(10 \mathrm{~mL})$. The mixture was sparged with argon for 20 minutes. Carbon tetrachloride (10 mmol, 20 equiv, $1 \mathrm{~mL}$ ) was then added. The reaction was irradiated with four blue LEDs lamps (Kessil H150B LED Grow Light), and let stir at room temperature with a fan to cool the reaction setup. After 18 hours, the reaction was concentrated then purified by silica gel column chromatography to obtain the titled compound as a white solid(112 $\mathrm{mg}, 98 \%)$. ${ }^{\mathbf{1}} \mathbf{H}$ NMR $\left(\mathbf{5 0 0} \mathbf{M H z}, \mathbf{C}_{\mathbf{6}} \mathbf{D}_{\mathbf{6}}\right) \delta 7.83(\mathrm{~d}, J=8.8 \mathrm{~Hz}, 2 \mathrm{H}), 6.66(\mathrm{~d}, J=8.9 \mathrm{~Hz}, 2 \mathrm{H}), 3.19(\mathrm{~s}, 3 \mathrm{H}), 3.09(\mathrm{t}, J=$ $6.7 \mathrm{~Hz}, 2 \mathrm{H}), 2.40(\mathrm{t}, J=7.2 \mathrm{~Hz}, 2 \mathrm{H}), 1.67$ (dt, $J=15.0,7.2 \mathrm{~Hz}, 2 \mathrm{H}), 1.47(\mathrm{dt}, J=14.0,6.8 \mathrm{~Hz}$, 2H). ${ }^{13}$ C NMR (126 MHz, $\left.\mathbf{C}_{6} \mathbf{D}_{6}\right) \delta$ 196.5, 163.2, 130.3, 130.1, 113.5, 54.5, 44.4, 36.7, 32.0, 21.4. IR (neat): 2952, 1666, 1600, 1576, 1510, 1460, 1443, 1418, 1375, 1309, 1293, 1261, 1240, 1171, 1114, 1027, 980, 836, 786, 736, $704 \mathrm{~cm}^{-1}$. HRMS (ESI): exact mass calculated for $[\mathrm{M}+\mathrm{H}]^{+}\left(\mathrm{C}_{12} \mathrm{H}_{16} \mathrm{ClO}_{2}\right)$ requires $\mathrm{m} / z$ 227.08334, found $\mathrm{m} / z 227.08298$. 


\section{Protocol for forming distally brominated ketones via PCET-mediated ring-opening of cyclic alcohols}
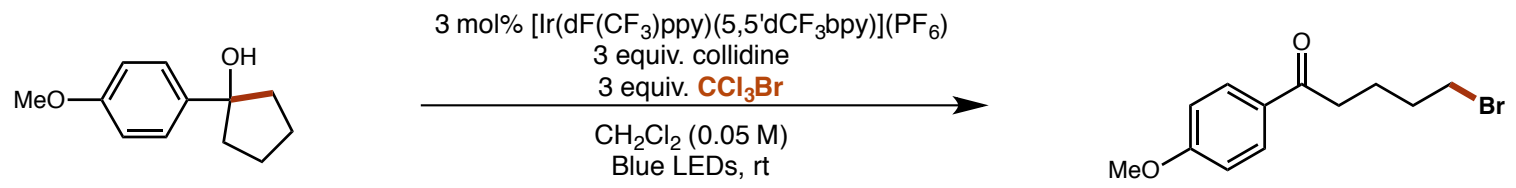

\section{5-bromo-1-(4-methoxyphenyl)pentan-1-one}

A screw cap culture tube $(16 \times 125 \mathrm{~mm})$ outfitted with a PTFE/silicone septa was charged with 1-(4-methoxyphenyl)cyclopentan-1-ol $(0.5 \mathrm{mmol}, 1$ equiv, $96 \mathrm{mg})$, redistilled collidine (1.5 mmol, 3 equiv, $198 \mu \mathrm{L})$, and $\left[\operatorname{Ir}\left(\mathrm{dF}\left(\mathrm{CF}_{3}\right) \text { ppy }\right)_{2}\left(5,5^{\prime} \mathrm{d}\left(\mathrm{CF}_{3}\right) \mathrm{bpy}\right)\right]\left(\mathrm{PF}_{6}\right)(0.015 \mathrm{mmol}, 0.03$ equiv, 16 $\mathrm{mg})$, The vial was then evacuated and backfilled with argon three times. Bromotrichloromethane (1.5 mmol, 3 equiv, $147 \mu \mathrm{L})$ and dry $\mathrm{CH}_{2} \mathrm{Cl}_{2}(10 \mathrm{~mL})$ were added. The reaction was irradiated with four blue LEDs lamps (Kessil H150B LED Grow Light), and let stir at room temperature with a fan to cool the reaction setup. After 24 hours, the reaction was concentrated then purified by silica gel column chromatography to obtain the titled compound (129 $\mathrm{mg}, 95 \%$ yield). ${ }^{1} \mathbf{H}$ NMR (500 MHz, C $\left.\mathbf{6}_{\mathbf{6}} \mathbf{D}_{6}\right) 7.83(\mathrm{~d}, J=8.8 \mathrm{~Hz}, 2 \mathrm{H}), 6.66(\mathrm{~d}, J=8.8 \mathrm{~Hz}, 2 \mathrm{H}), 3.19$ (s, 3H), 2.93 (t, $J=6.8 \mathrm{~Hz}, 2 \mathrm{H}), 2.38(\mathrm{t}, J=7.1 \mathrm{~Hz}, 2 \mathrm{H}), 1.65(\mathrm{p}, J=7.1 \mathrm{~Hz}, 2 \mathrm{H}), 1.54(\mathrm{p}, J=6.8 \mathrm{~Hz}, 2 \mathrm{H})$. ${ }^{13}$ C NMR (126 MHz, C $\left.\mathbf{C}_{6}\right) \delta 196.4,163.2,130.3,130.1,113.5,54.5,36.6,33.0 .32 .1,22.7$. IR (neat): 2949, 1665, 1600, 1513, 1459, 1438, 1406, 1375, 1309, 1266, 1226, 1186, 1172, 1115 , 1027, 982, 834, 778, $737 \mathrm{~cm}^{-1}$. HRMS (ESI): exact mass calculated for $[\mathrm{M}+\mathrm{H}]^{+}\left(\mathrm{C}_{12} \mathrm{H}_{16} \mathrm{BrO}_{2}\right)$ requires $\mathrm{m} / \mathrm{z} 271.03282$, found $\mathrm{m} / \mathrm{z} 271.03285$. 


\section{Protocol for Baeyer-Villiger oxidation of ketone product}

To verify that the PMP-ketone products can be easily derivatized via Baeyer-Villiger oxidation to furnish free carboxylic acid, 1-(4-methoxyphenyl)propan-1-one was subjected to classical Baeyer-Villiger conditions, the procedure and result of which are outlined below.<smiles>CCC(=O)c1ccc(OC)cc1</smiles>

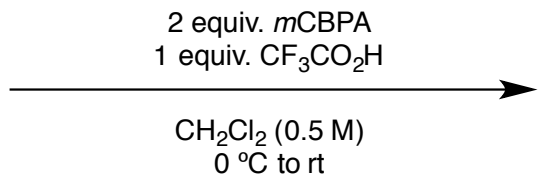<smiles>CCC(=O)Oc1ccc(OC)cc1</smiles>

\section{4-methoxyphenyl propionate}

A screw-cap 2-dram vial was charged with 1-(4-methoxyphenyl)propan-1-one (1.0 mmol, 1.0 equiv, $164 \mathrm{mg})$ and 3-chlorobenzoperoxoic acid ( 70\% purity, $493 \mathrm{mg} ; 2.0 \mathrm{mmol}, 2.0$ equiv in pure $m \mathrm{CPBA}$ ). The solids were dissolved in $2 \mathrm{~mL}$ of $\mathrm{CH}_{2} \mathrm{Cl}_{2}$ to form a suspension, which was stirred vigorouly while cooled with an ice bath. Trifluoroacetic acid $(1.0 \mathrm{mmol}, 1.0$ equiv, $75 \mu \mathrm{L})$ was added slowly via syringe. The vial was left in the ice bath overnight, during which time the reaction warmed up to room tempurature. The crude material was washed in saturated $\mathrm{NaHCO}_{3}$, extracted with $\mathrm{CH}_{2} \mathrm{Cl}_{2}$, concentrate to small volume, and purified by silica gel column chromatography to afford the titled compound as a colorless liquid (147 mg, 81\%). Spectra are consistent with reported literature values. ${ }^{12}$ 


\section{${ }^{1} \mathrm{H}$ and ${ }^{13} \mathrm{C}$ NMR Spectra of Products}

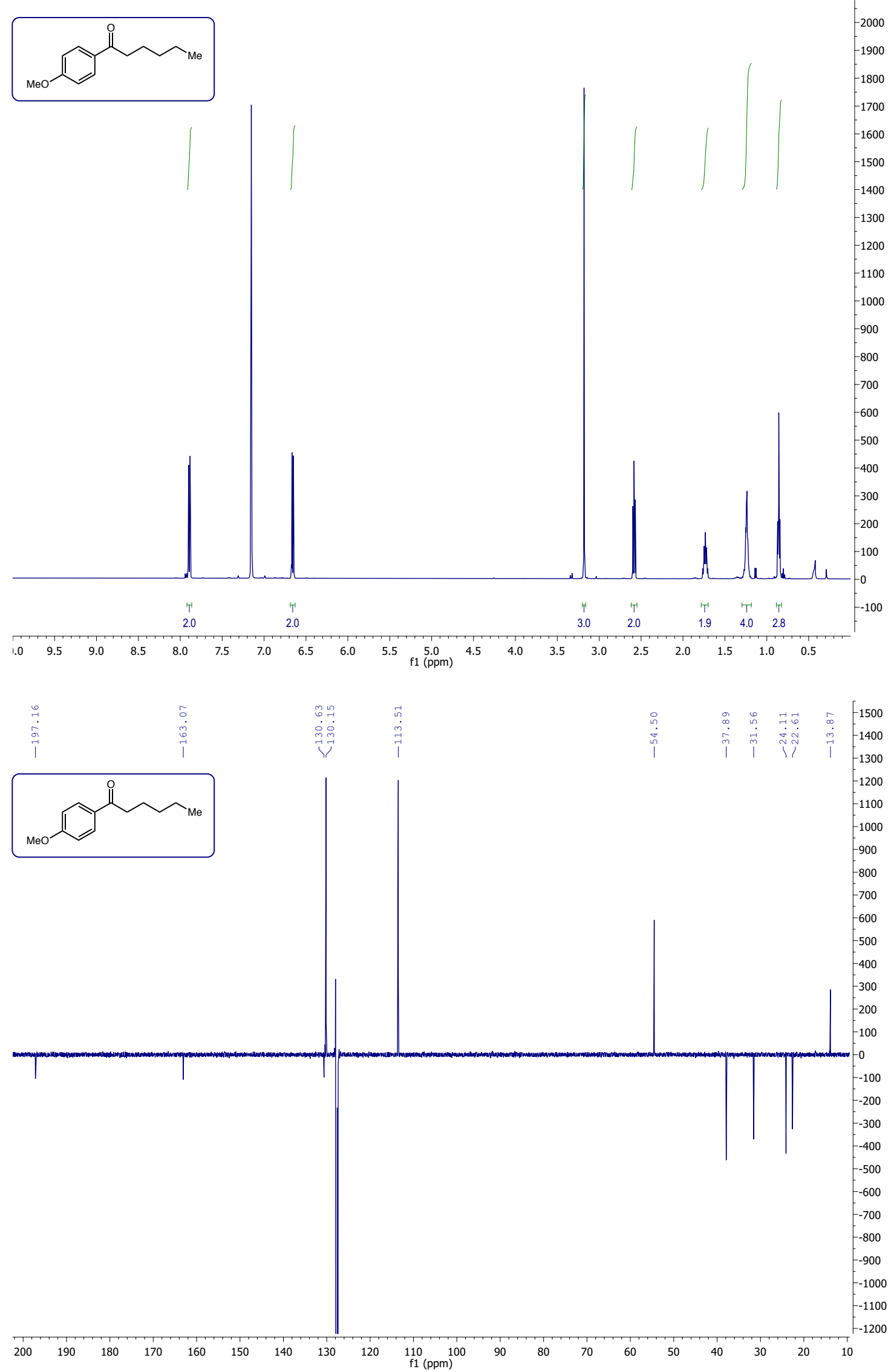




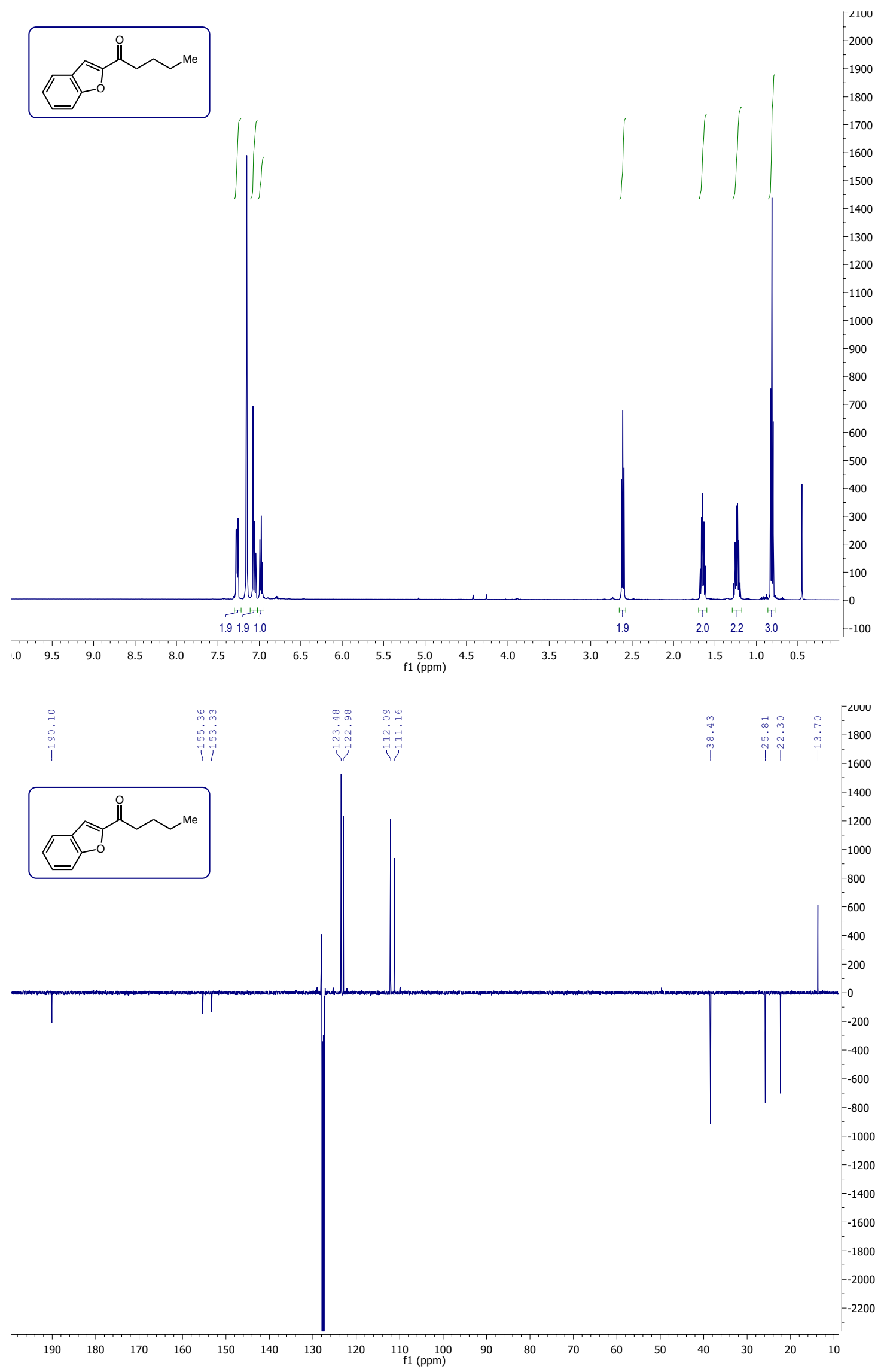




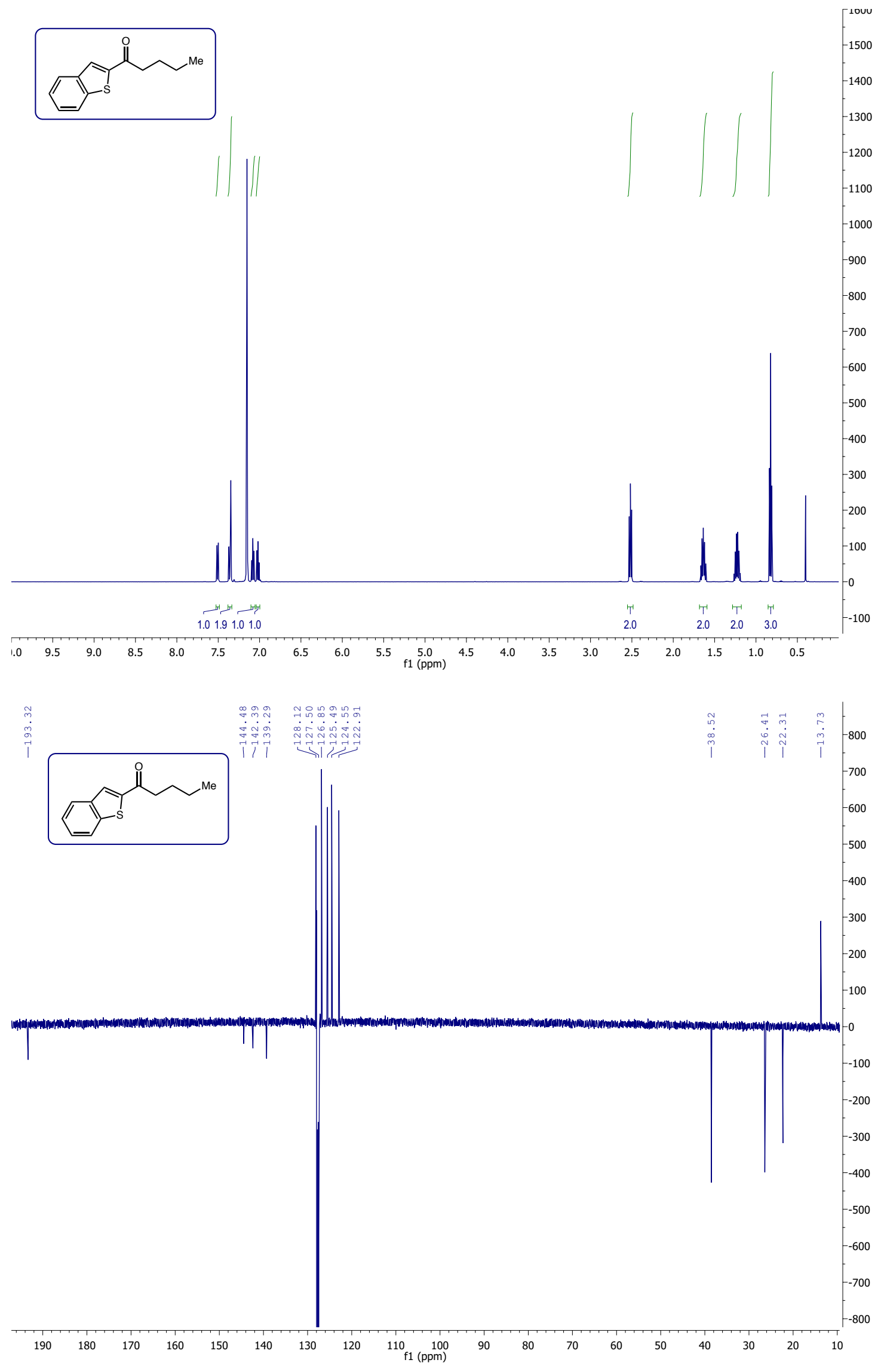




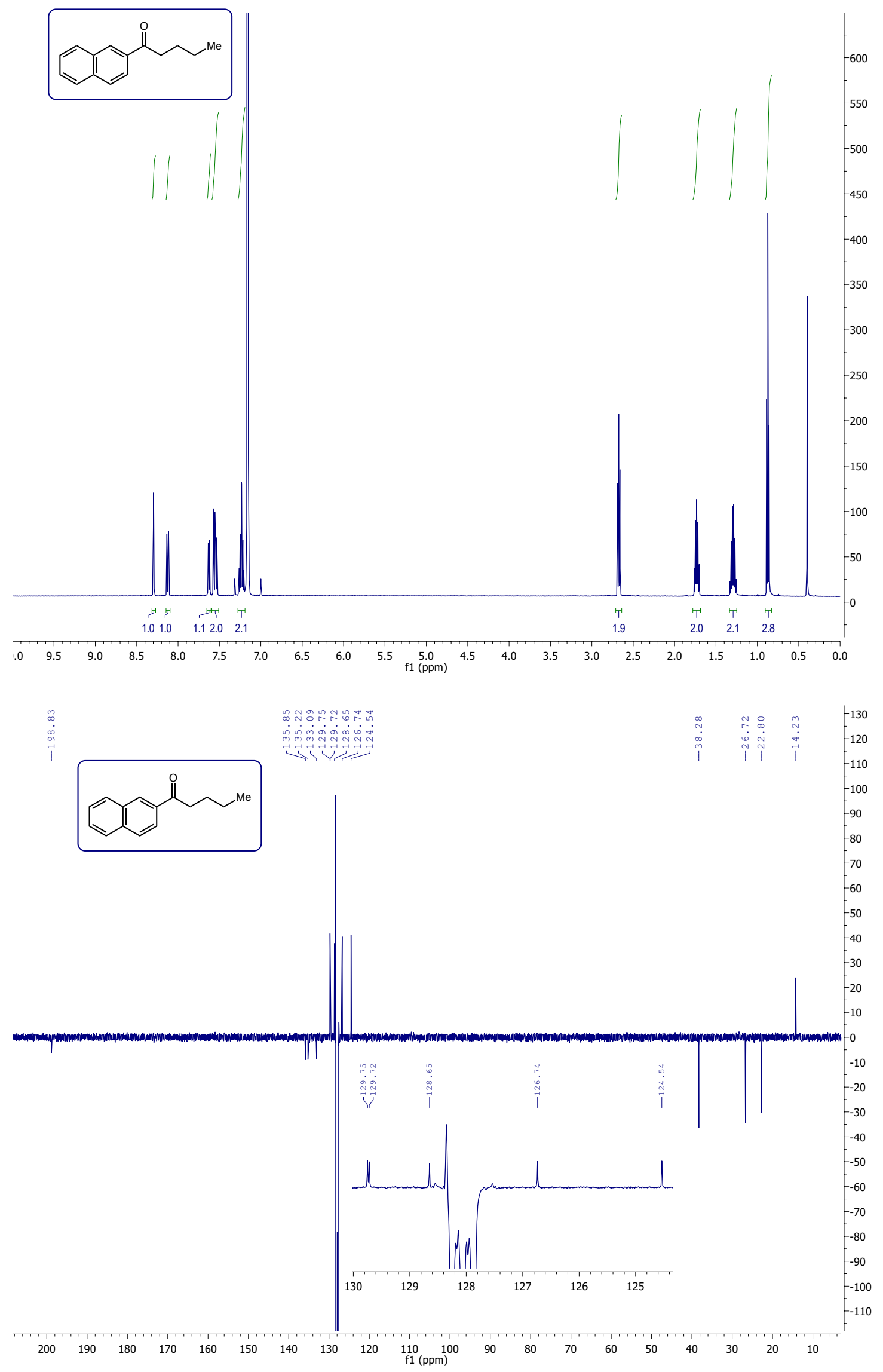




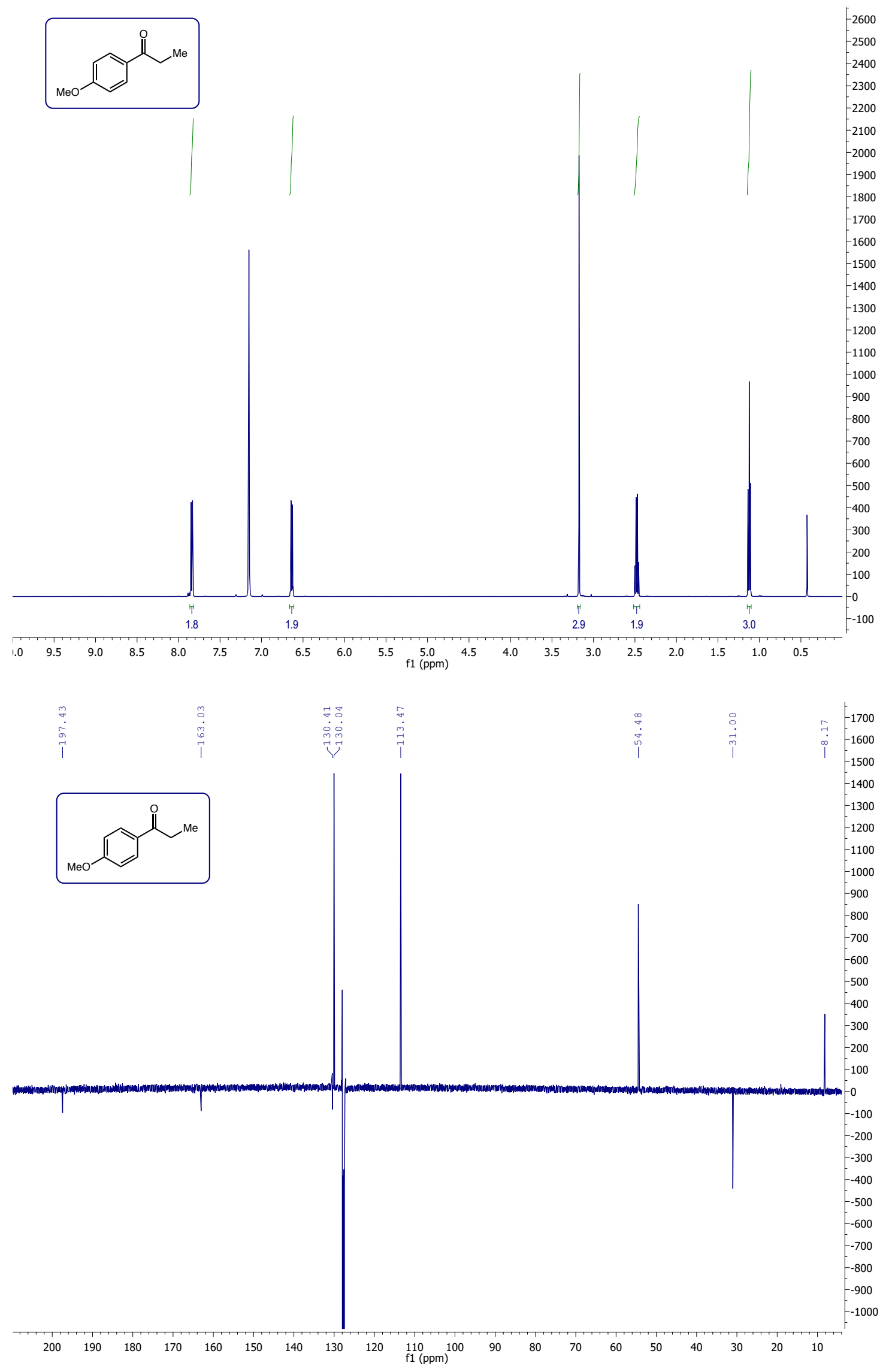



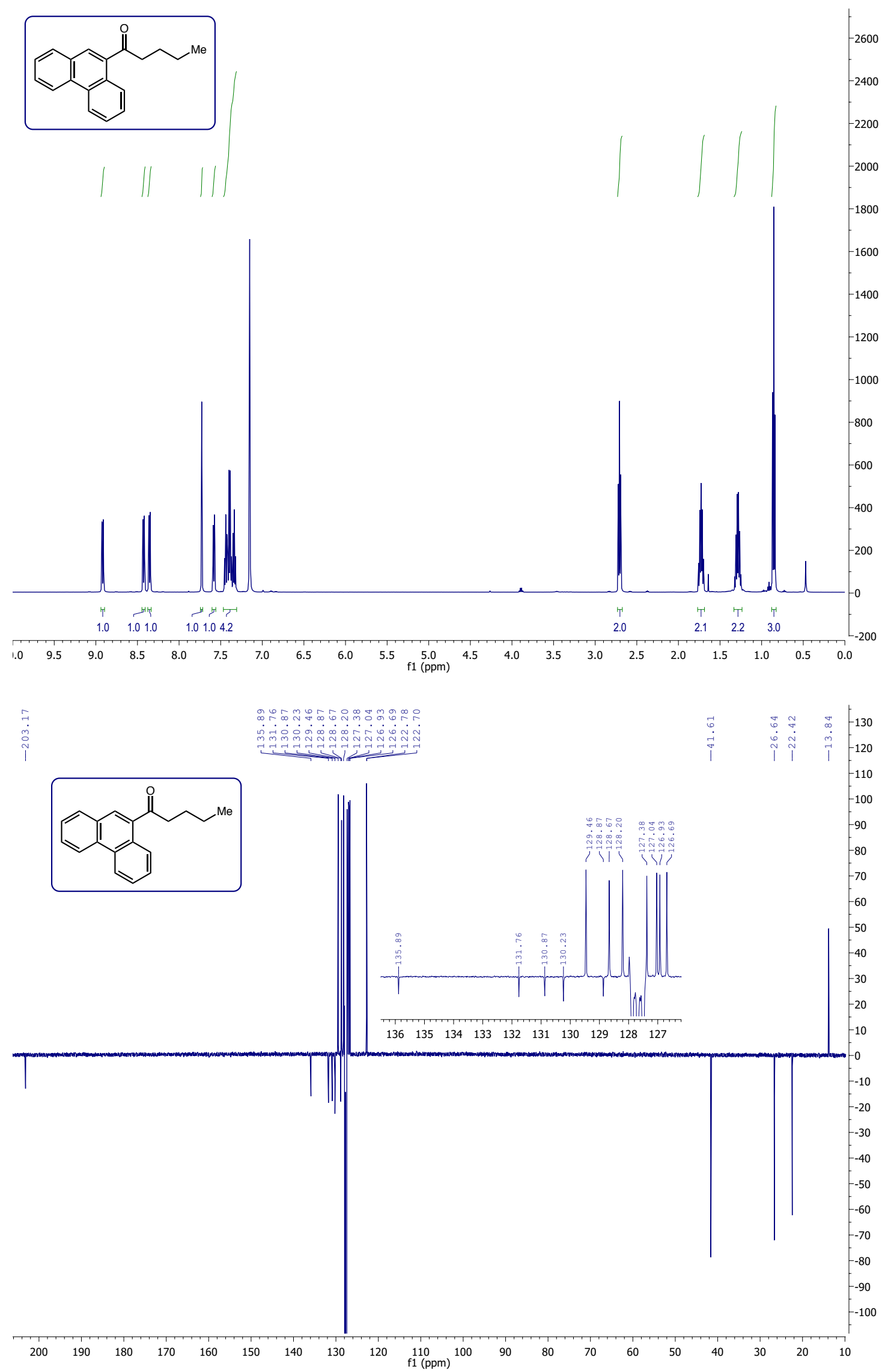

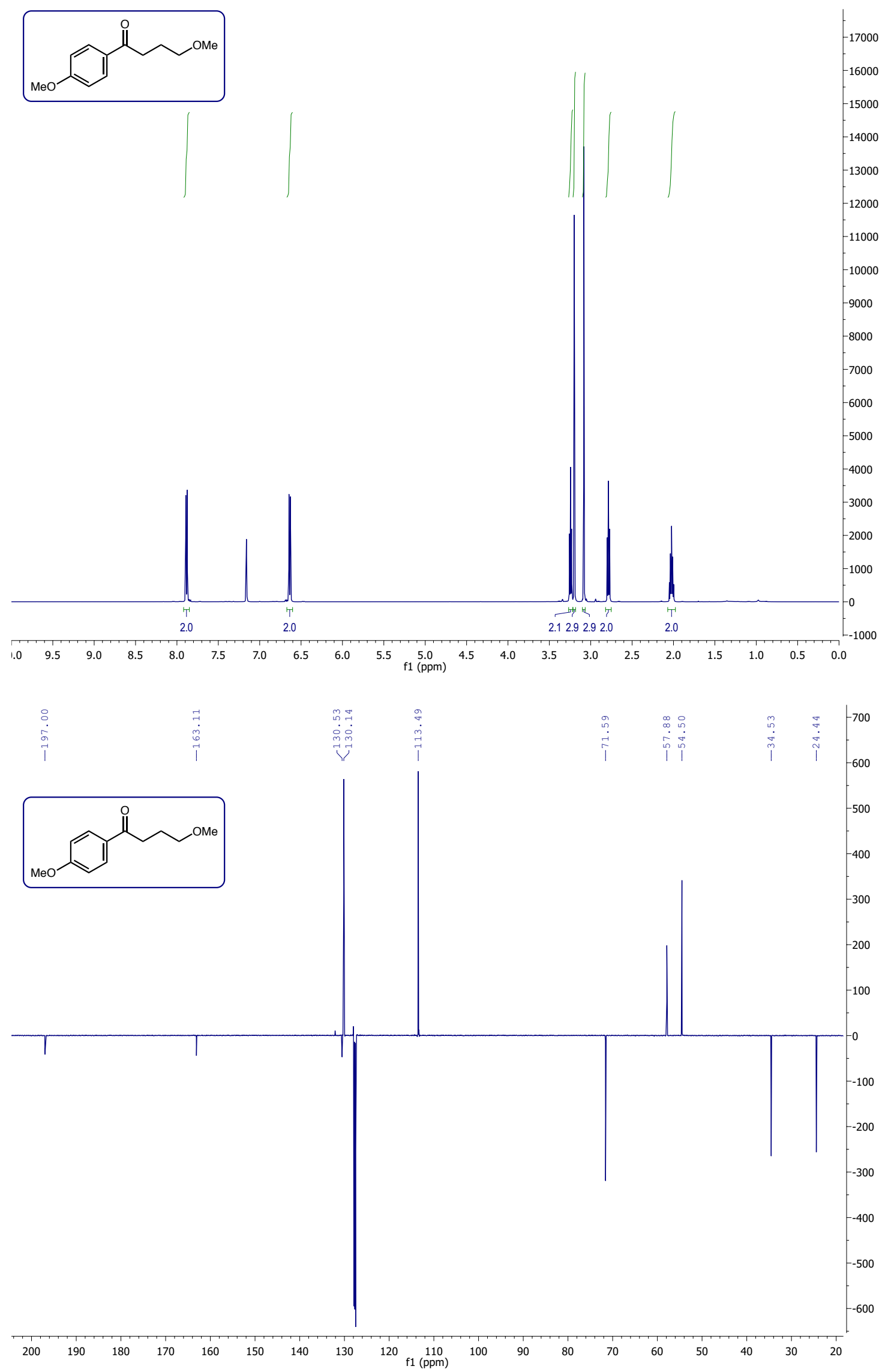

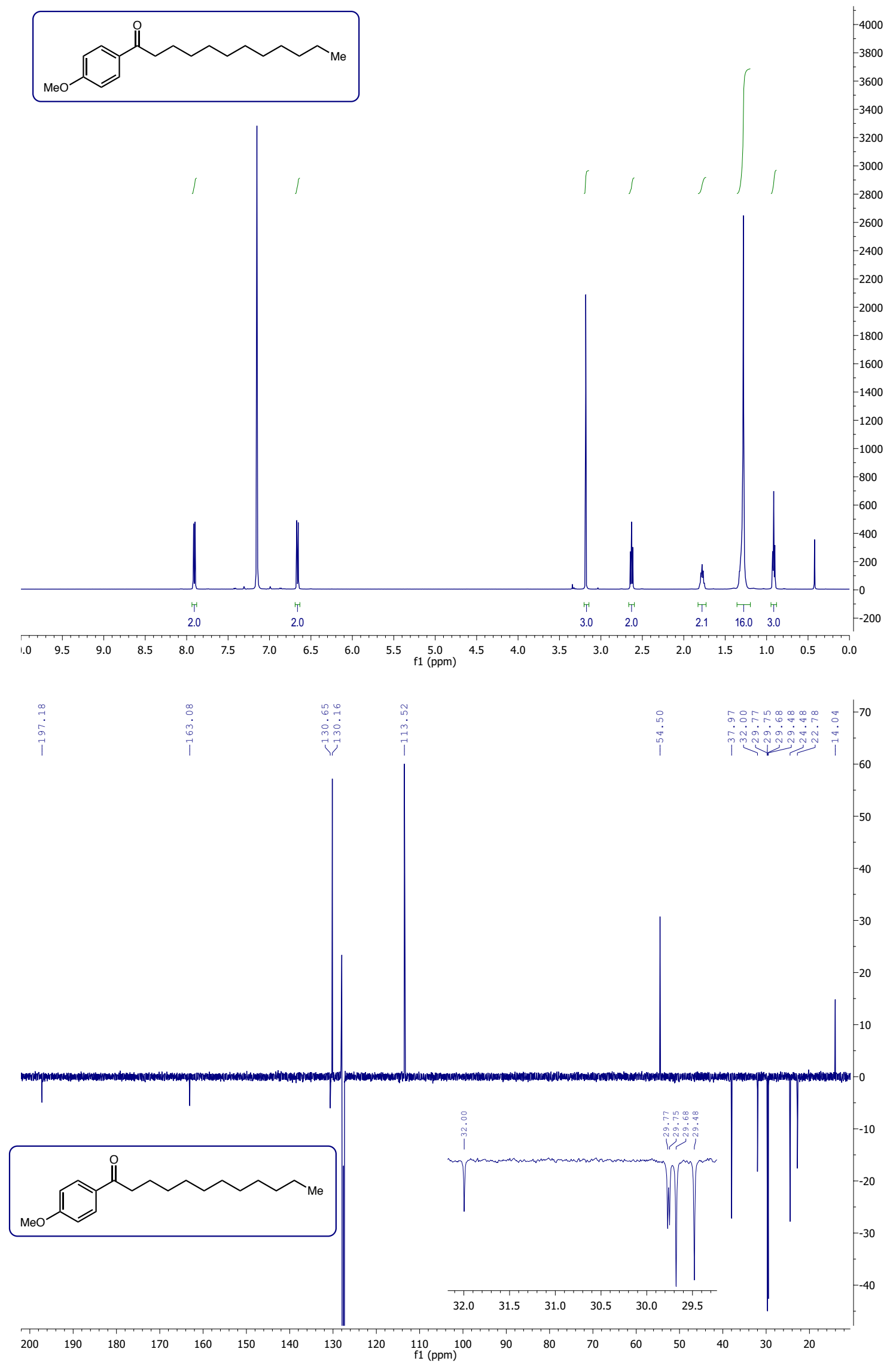

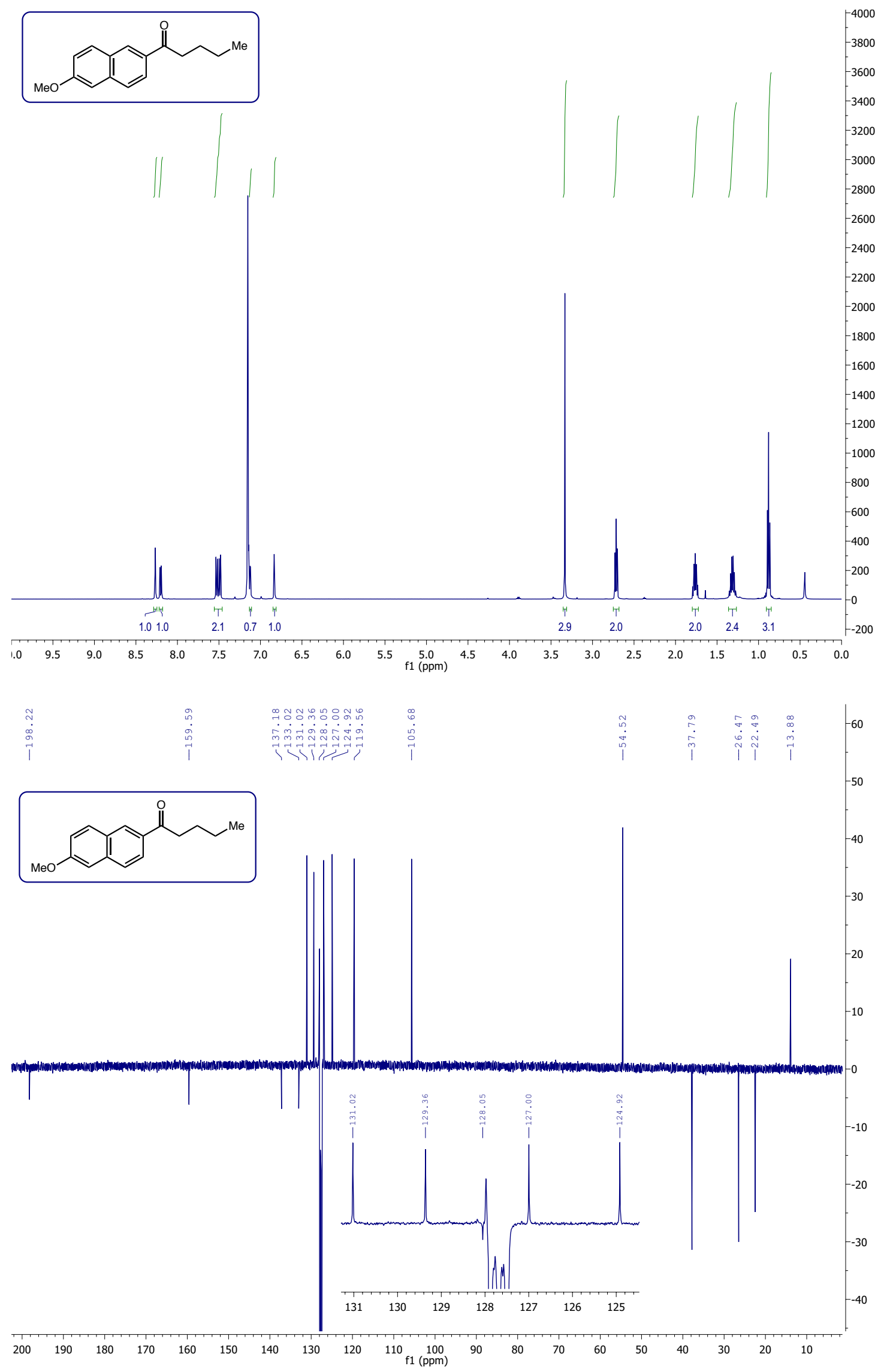

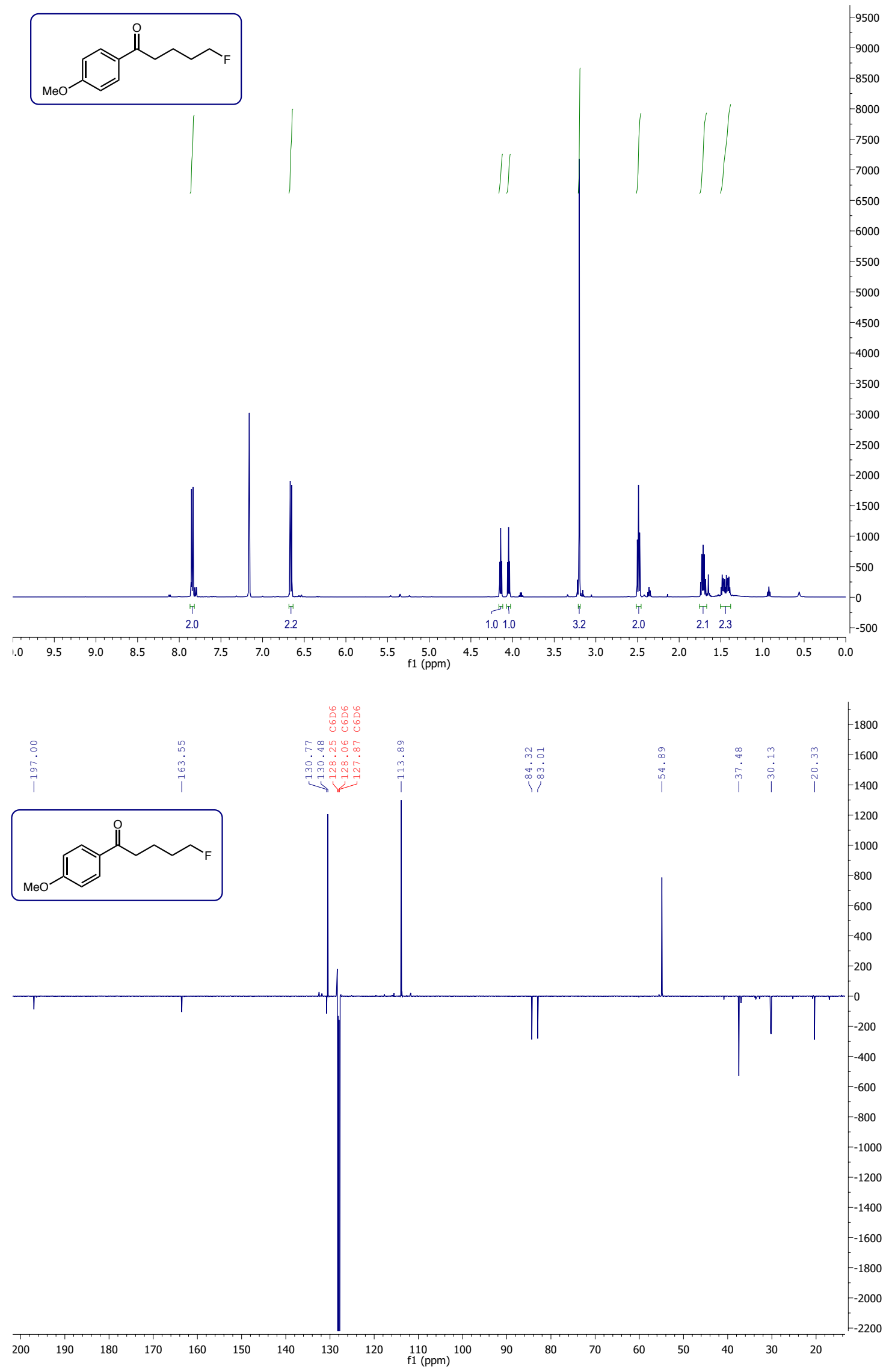

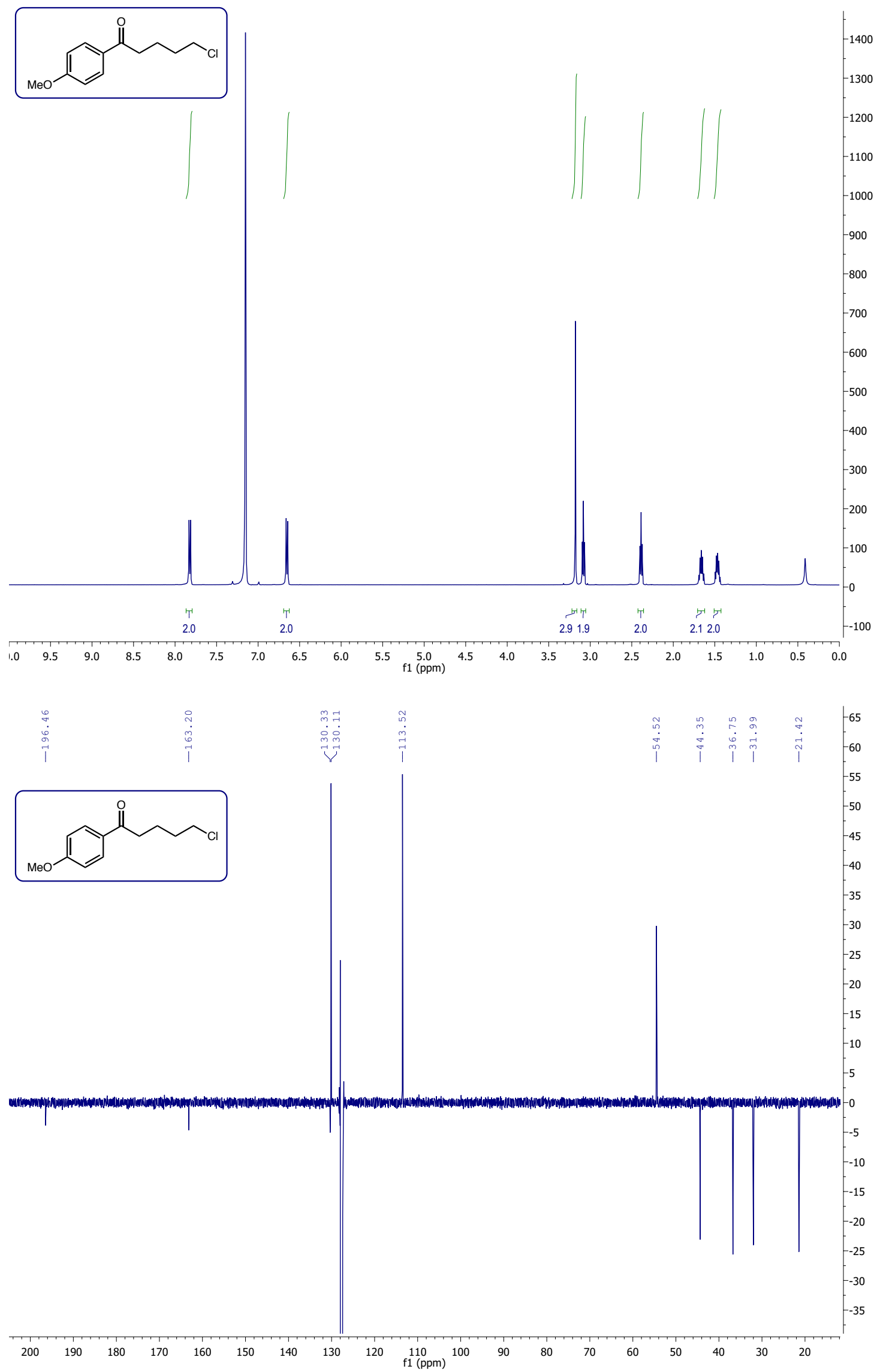

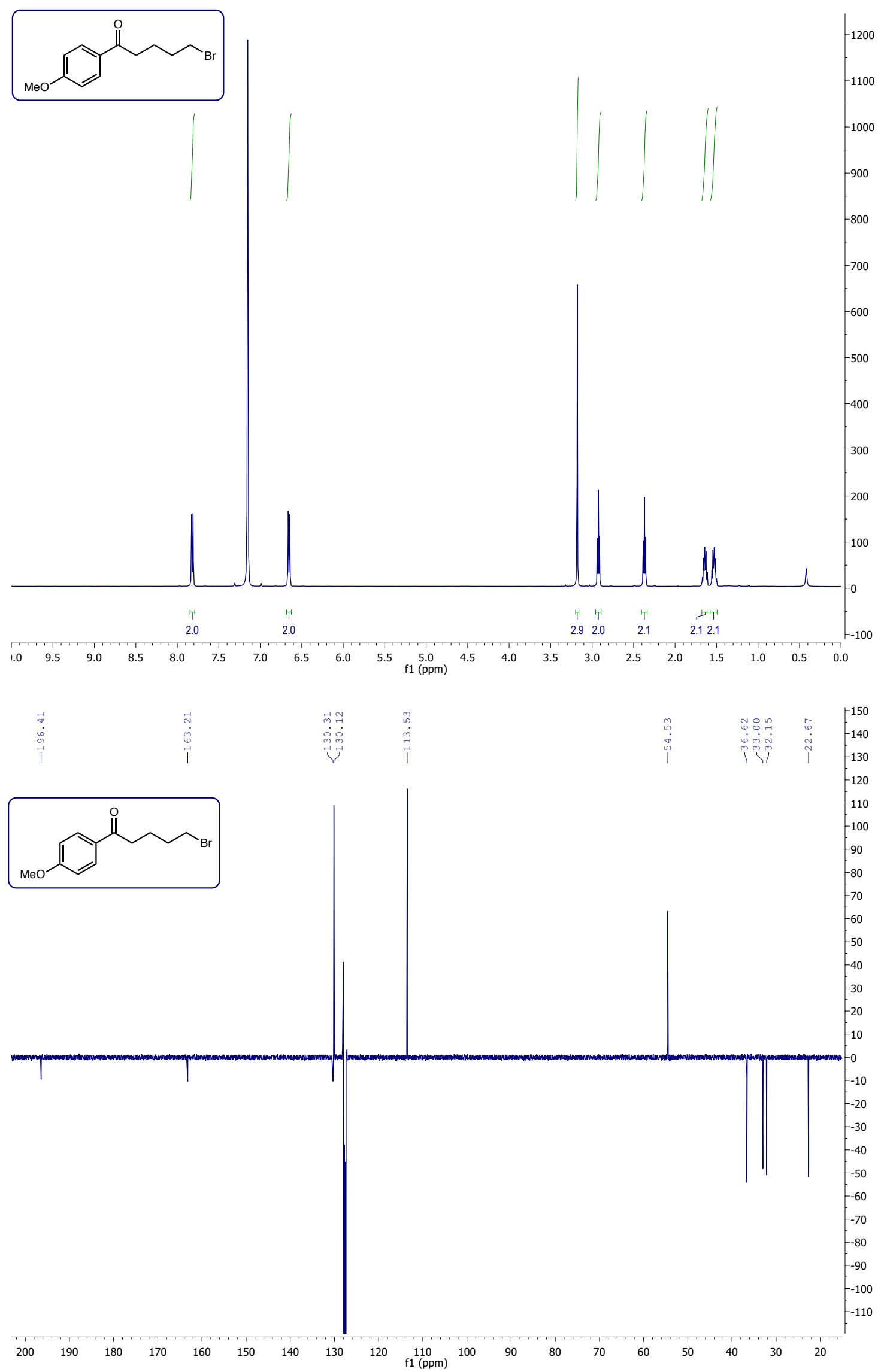

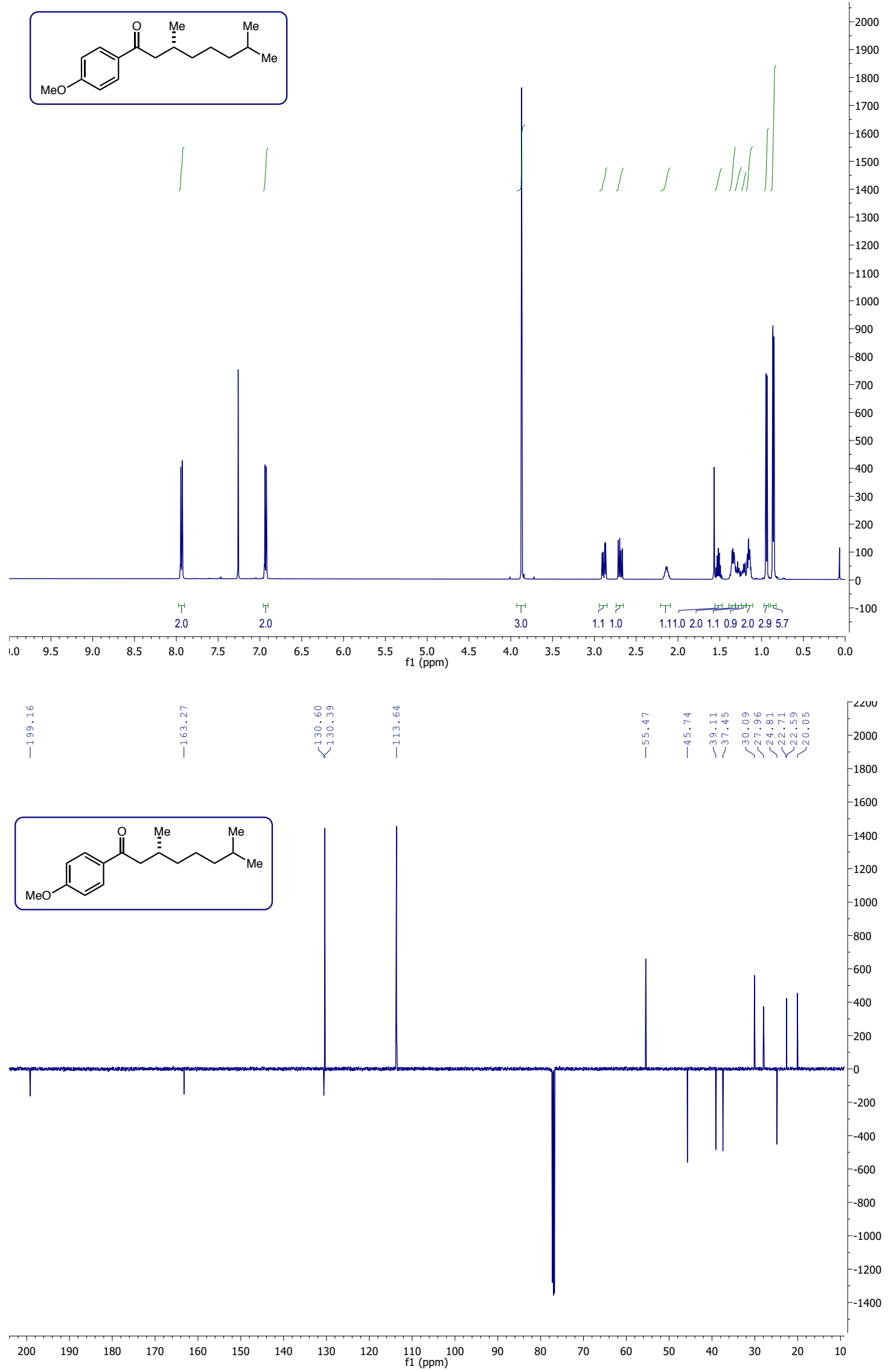

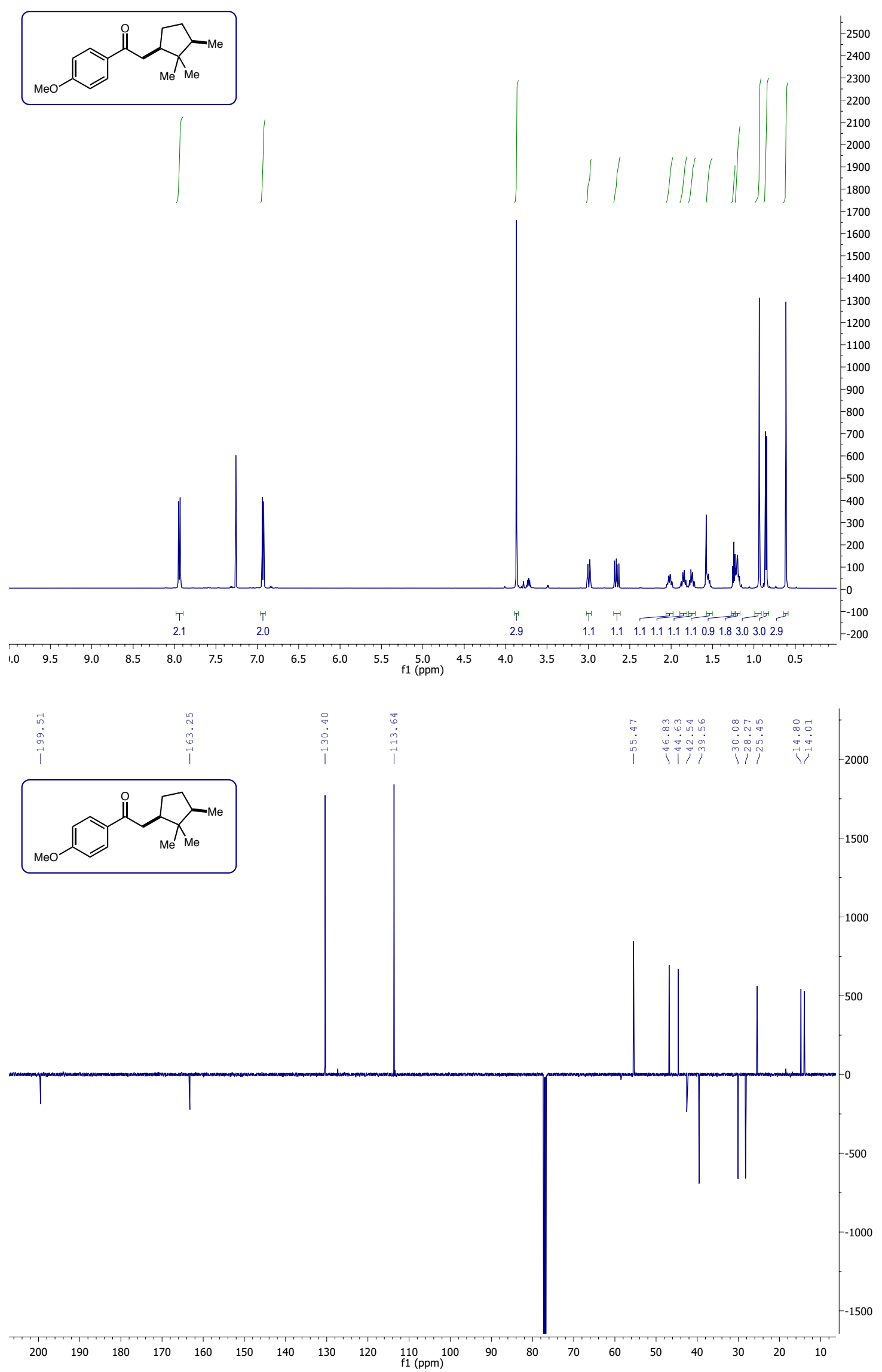

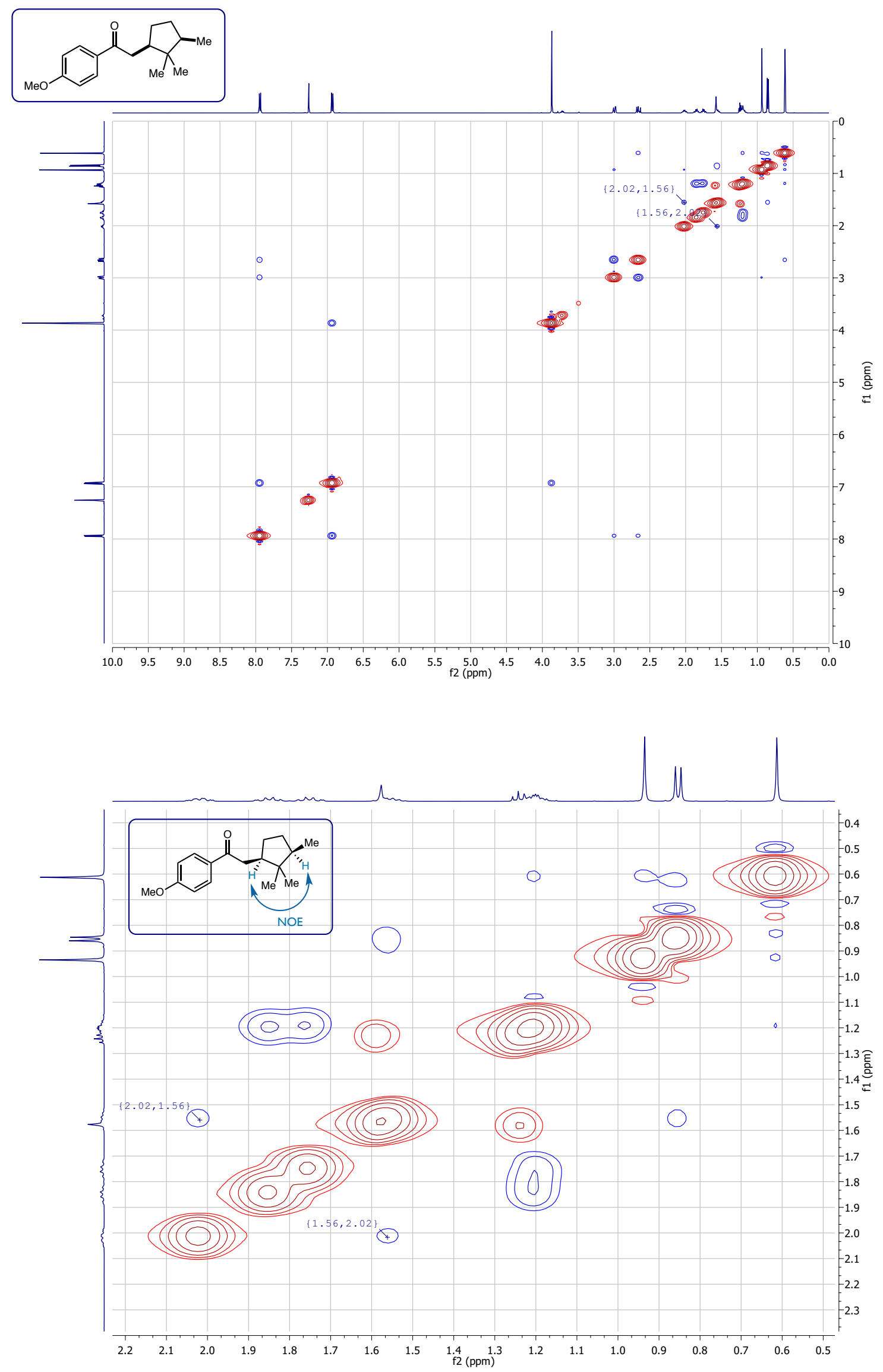

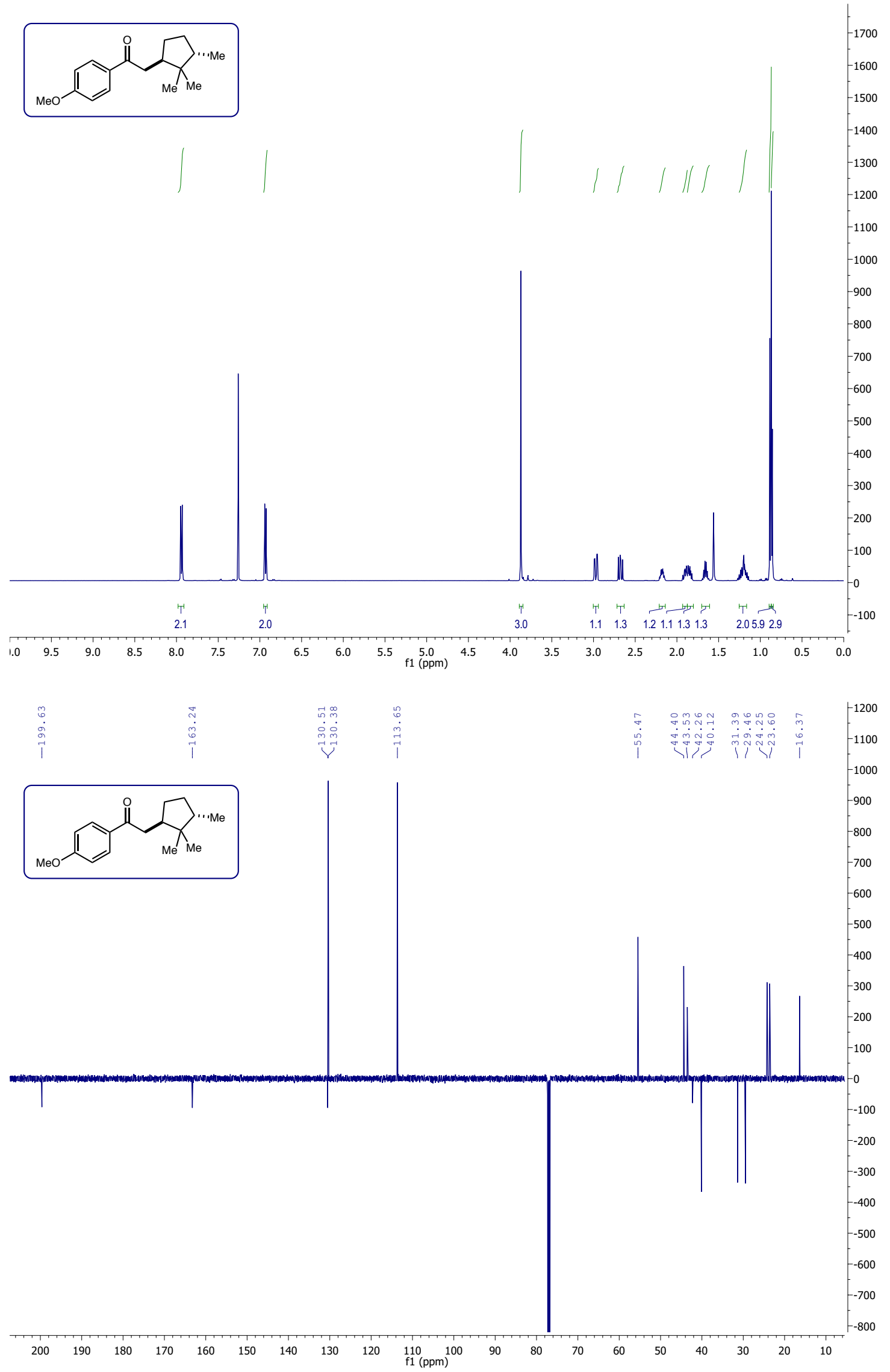


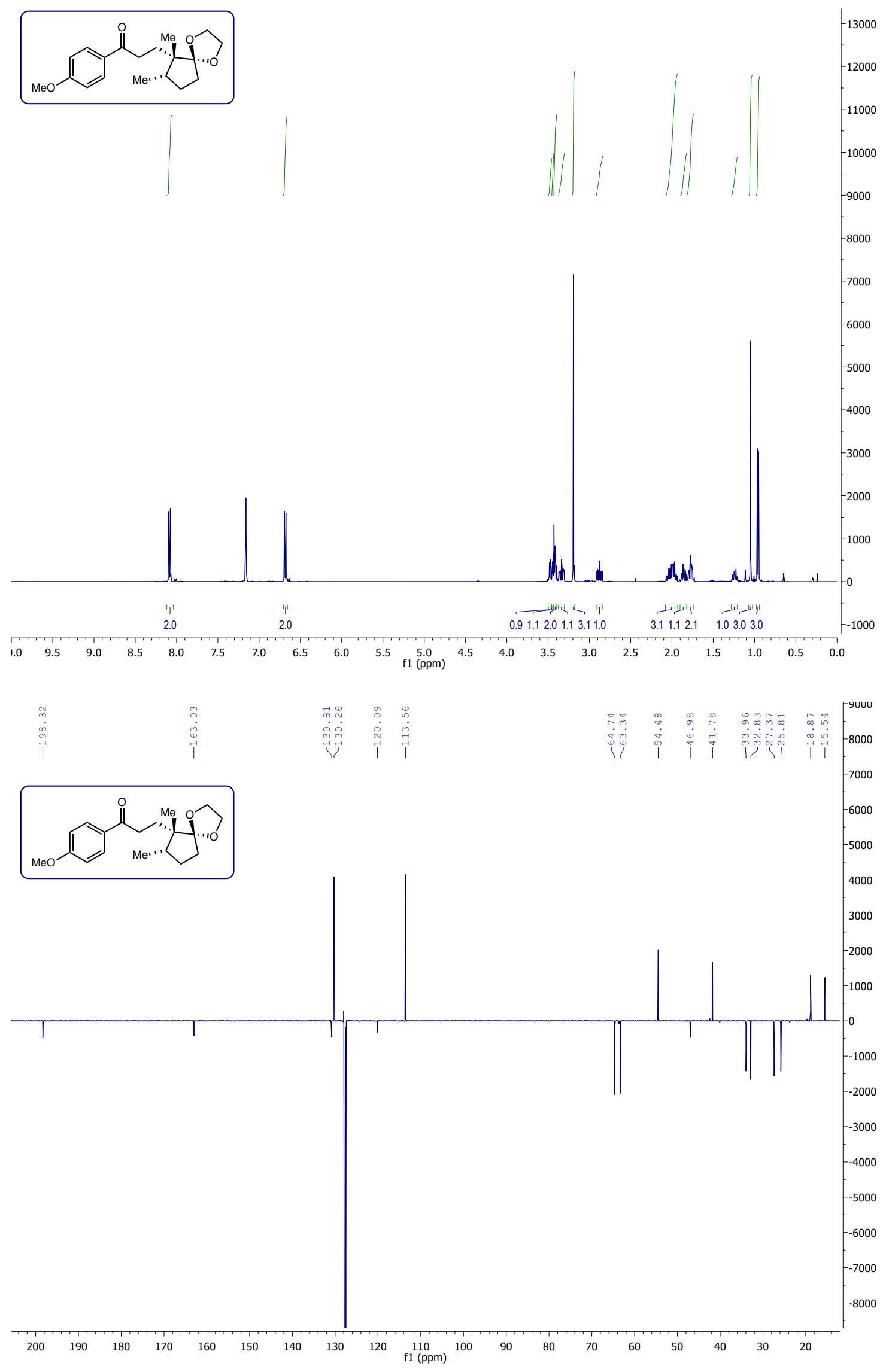



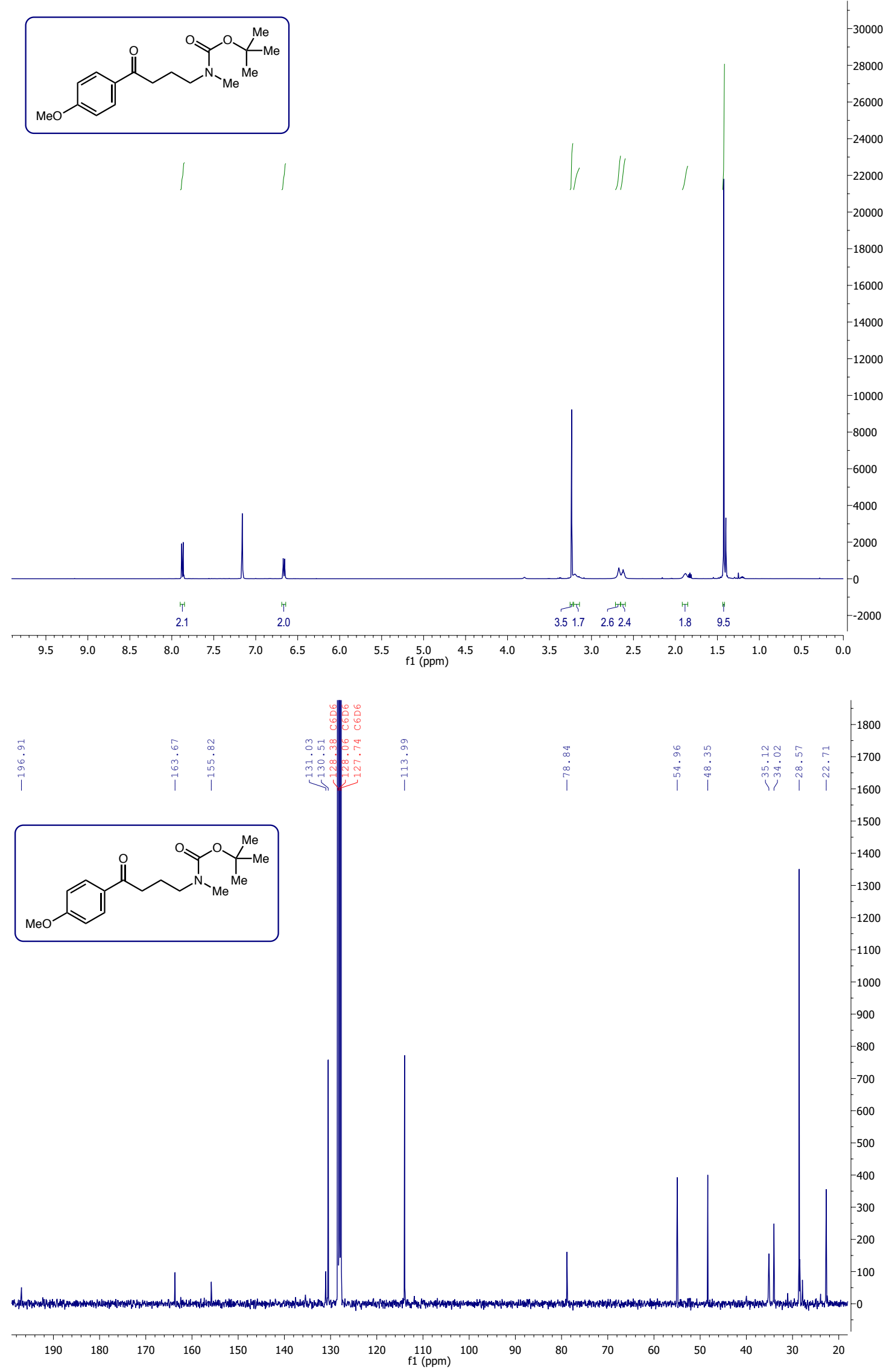


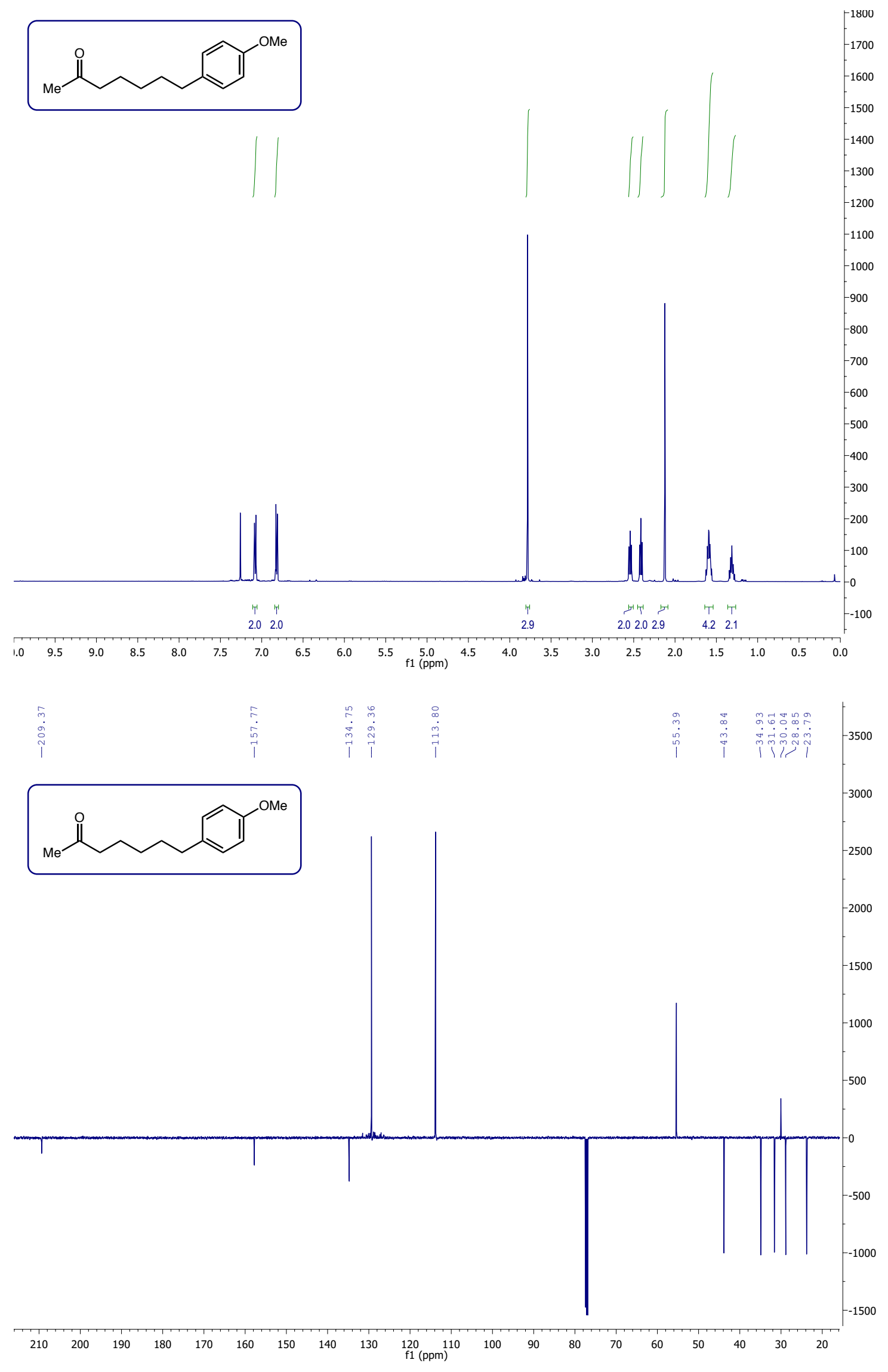




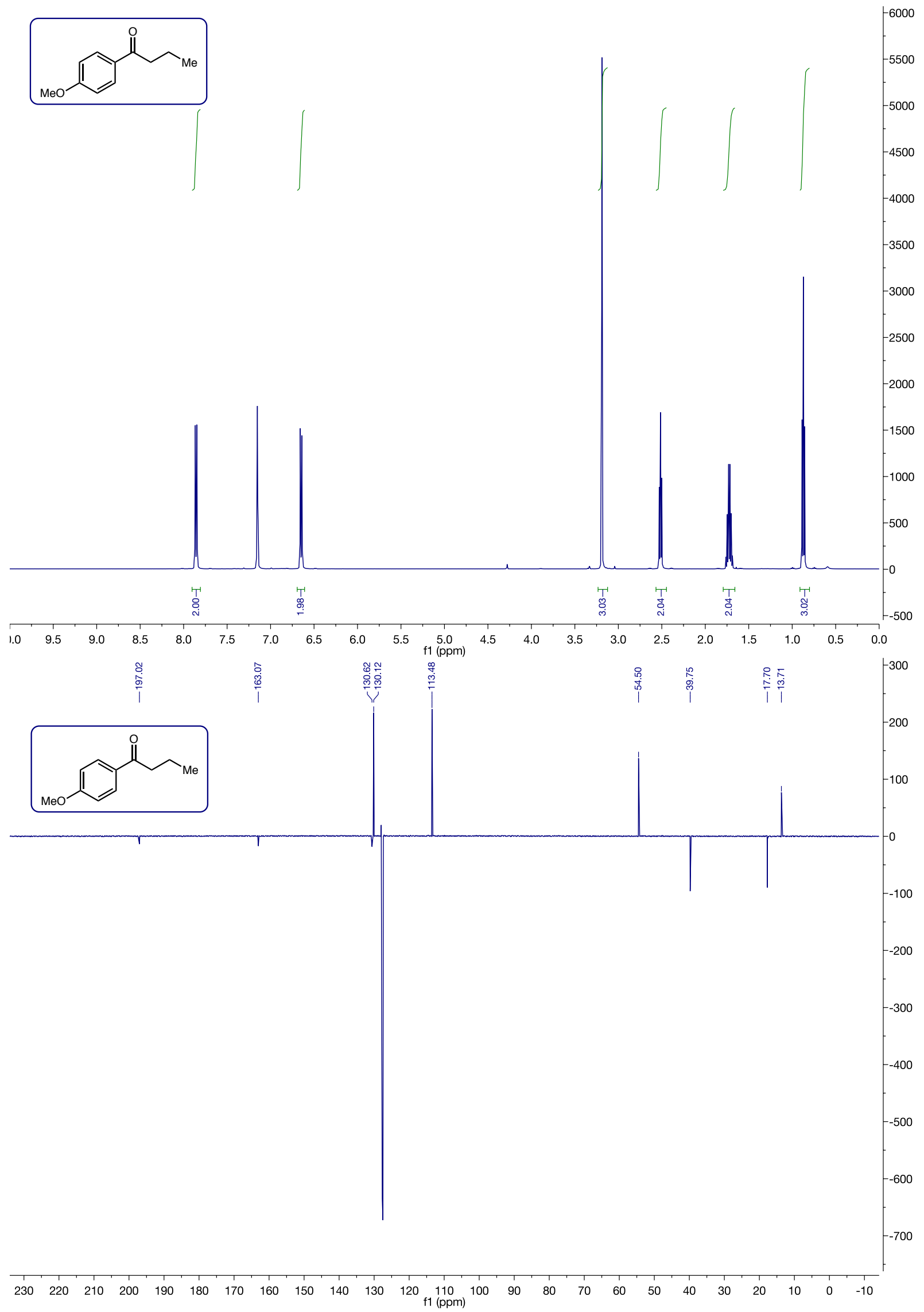




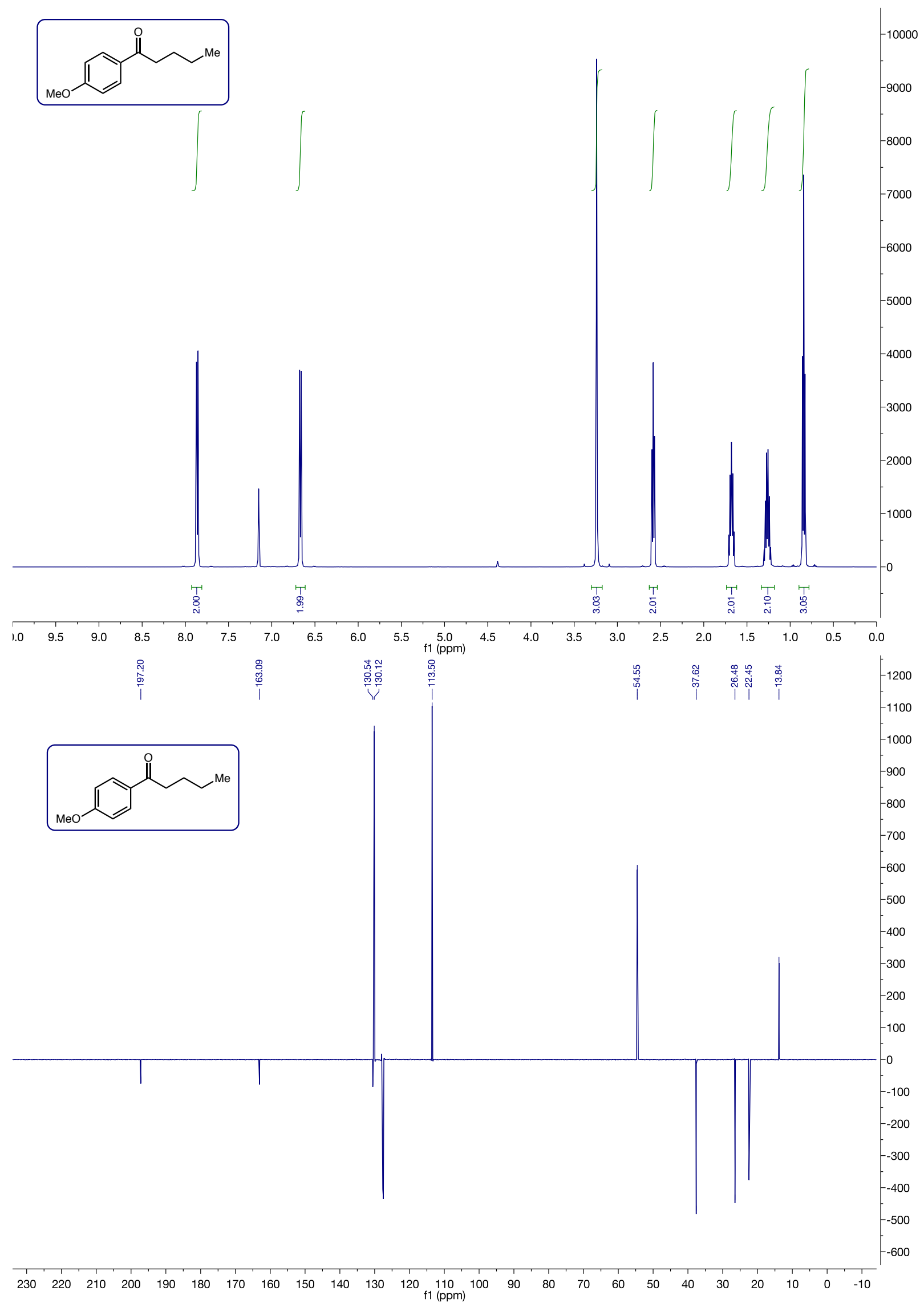




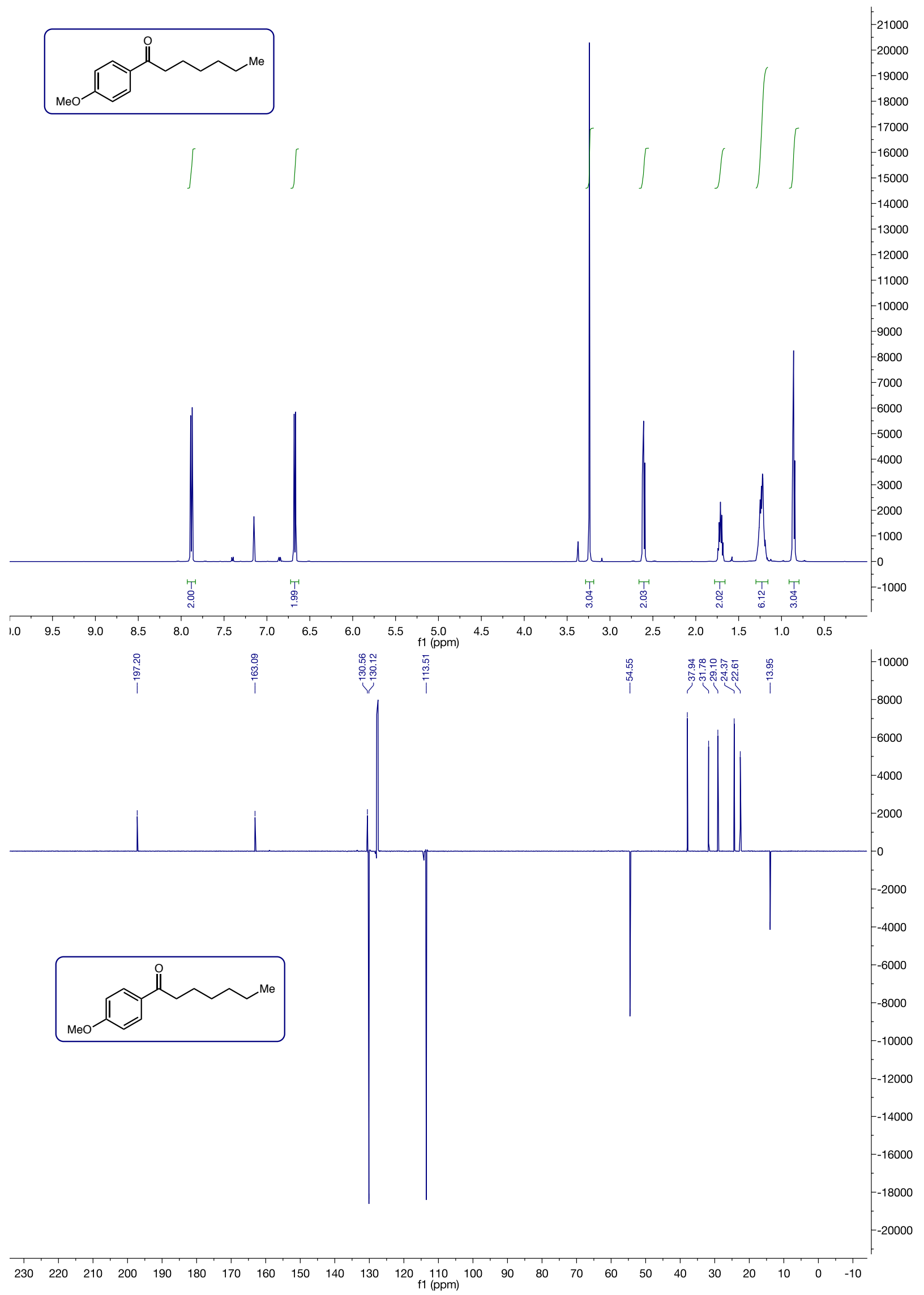




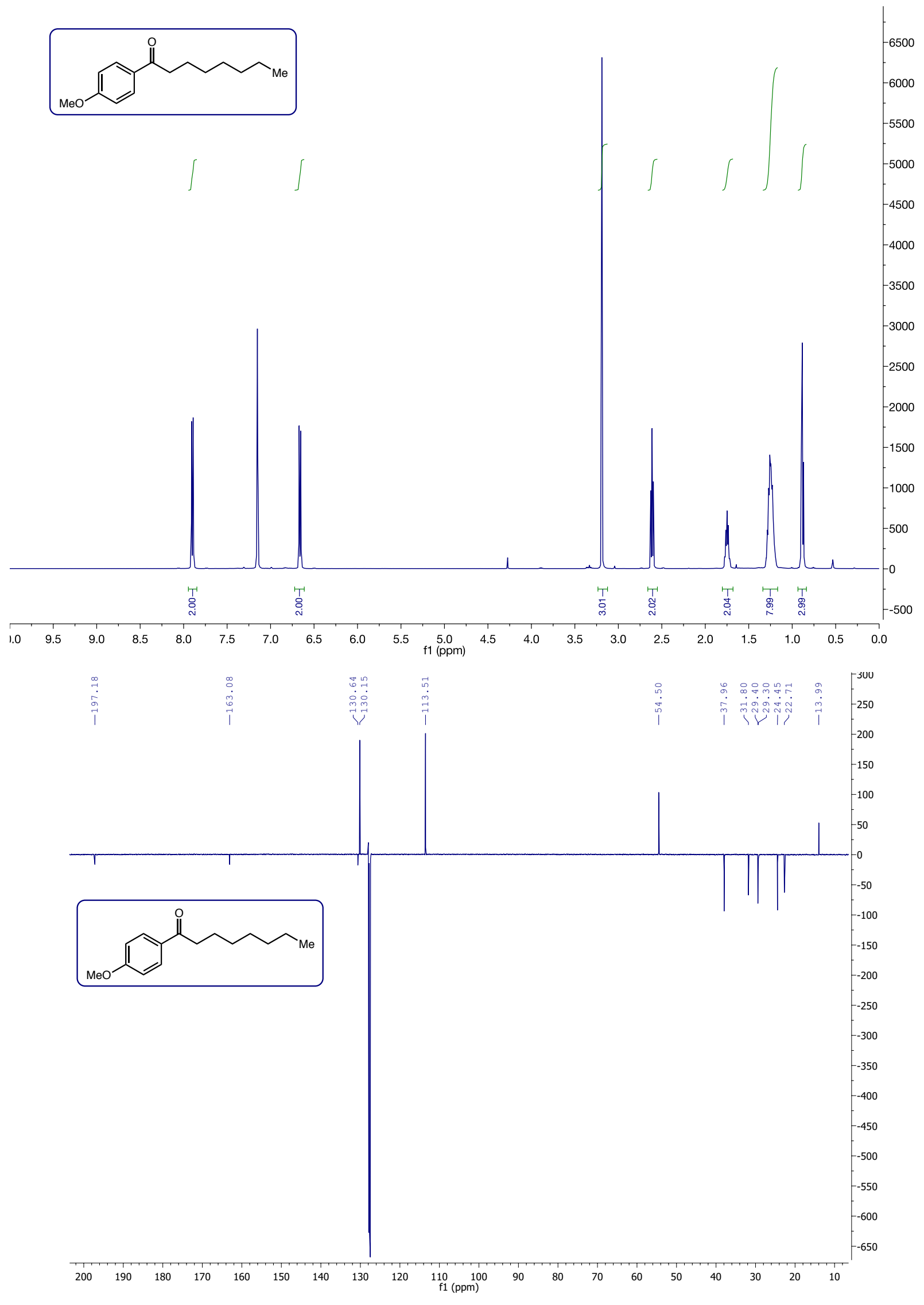




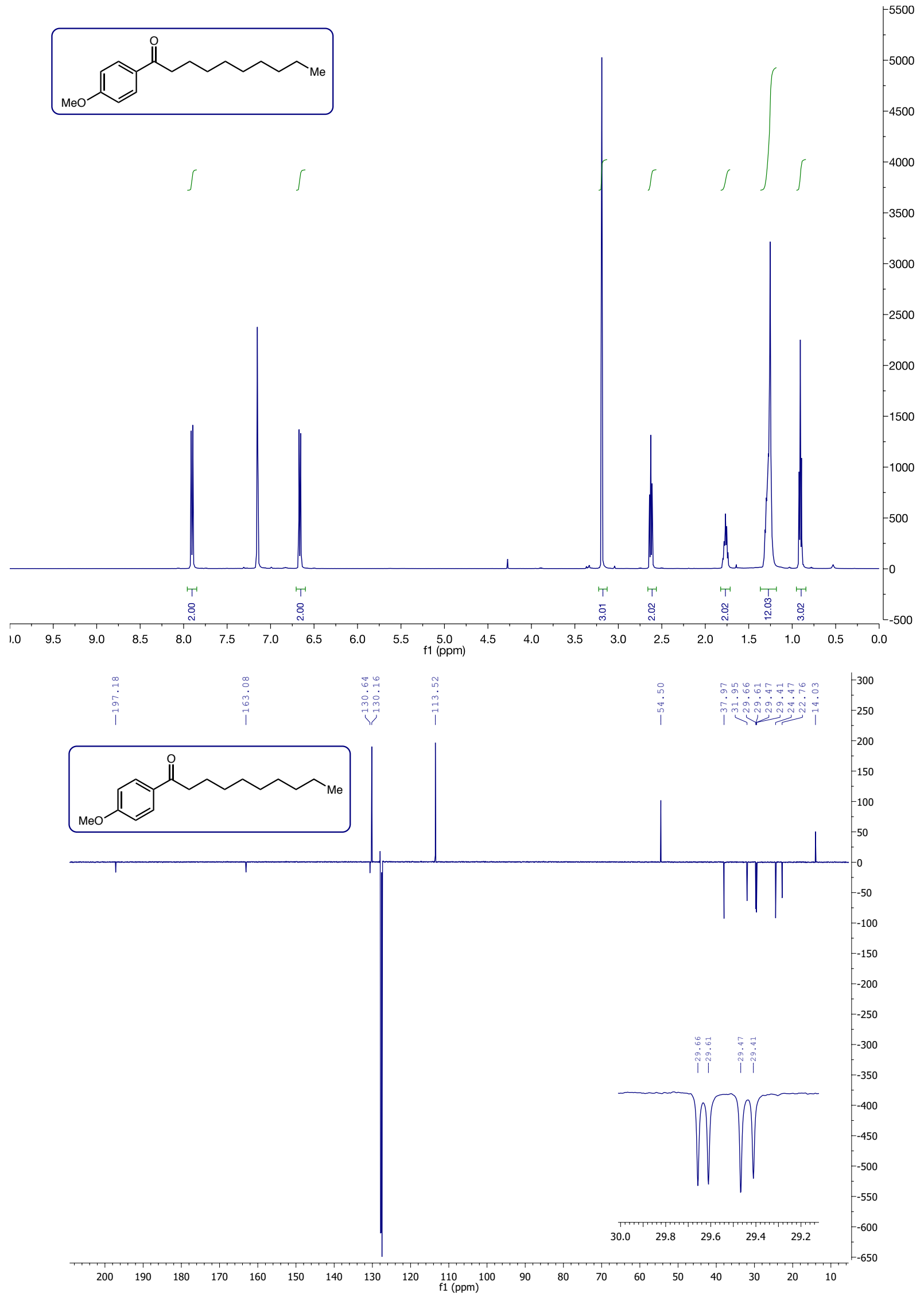




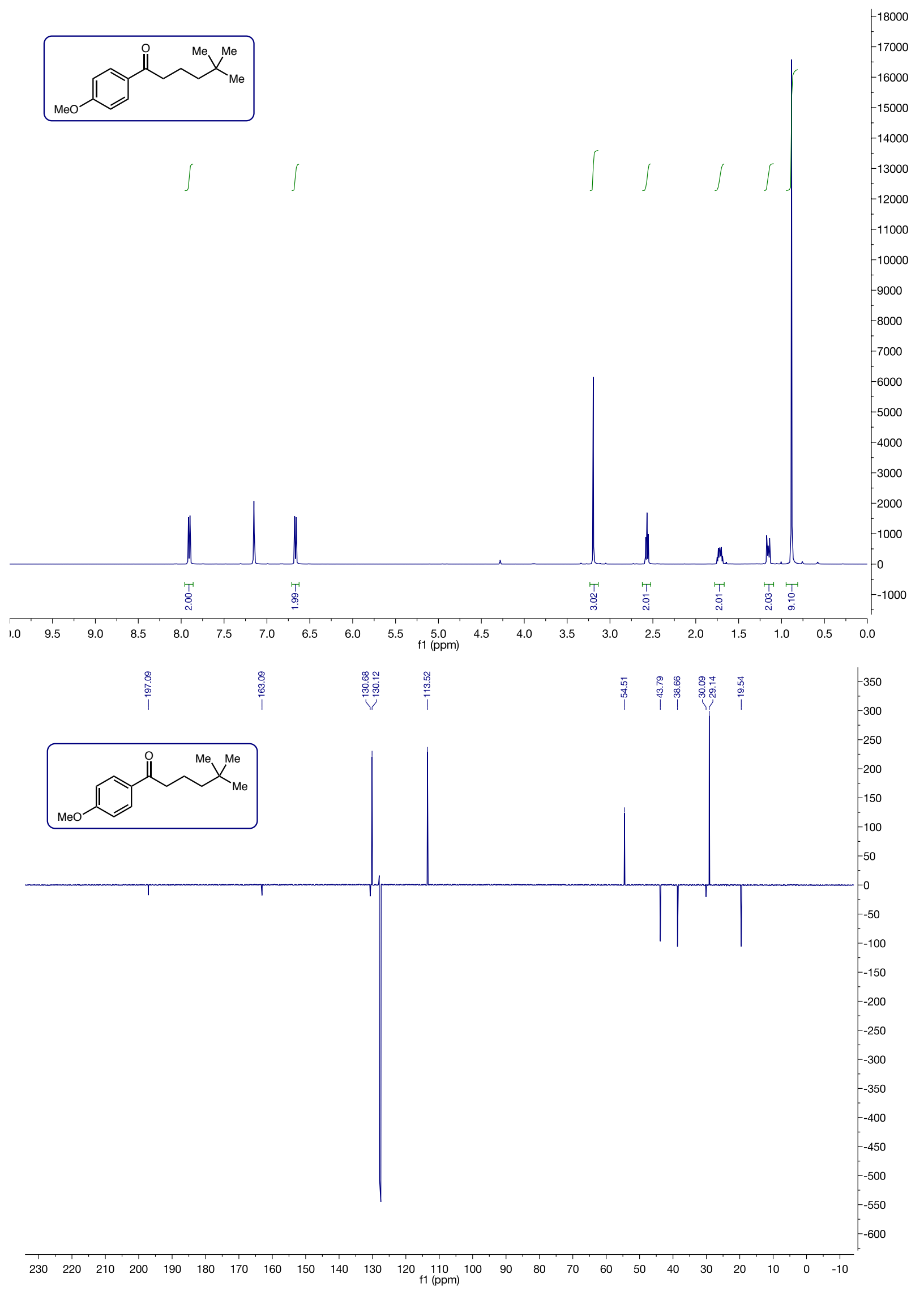




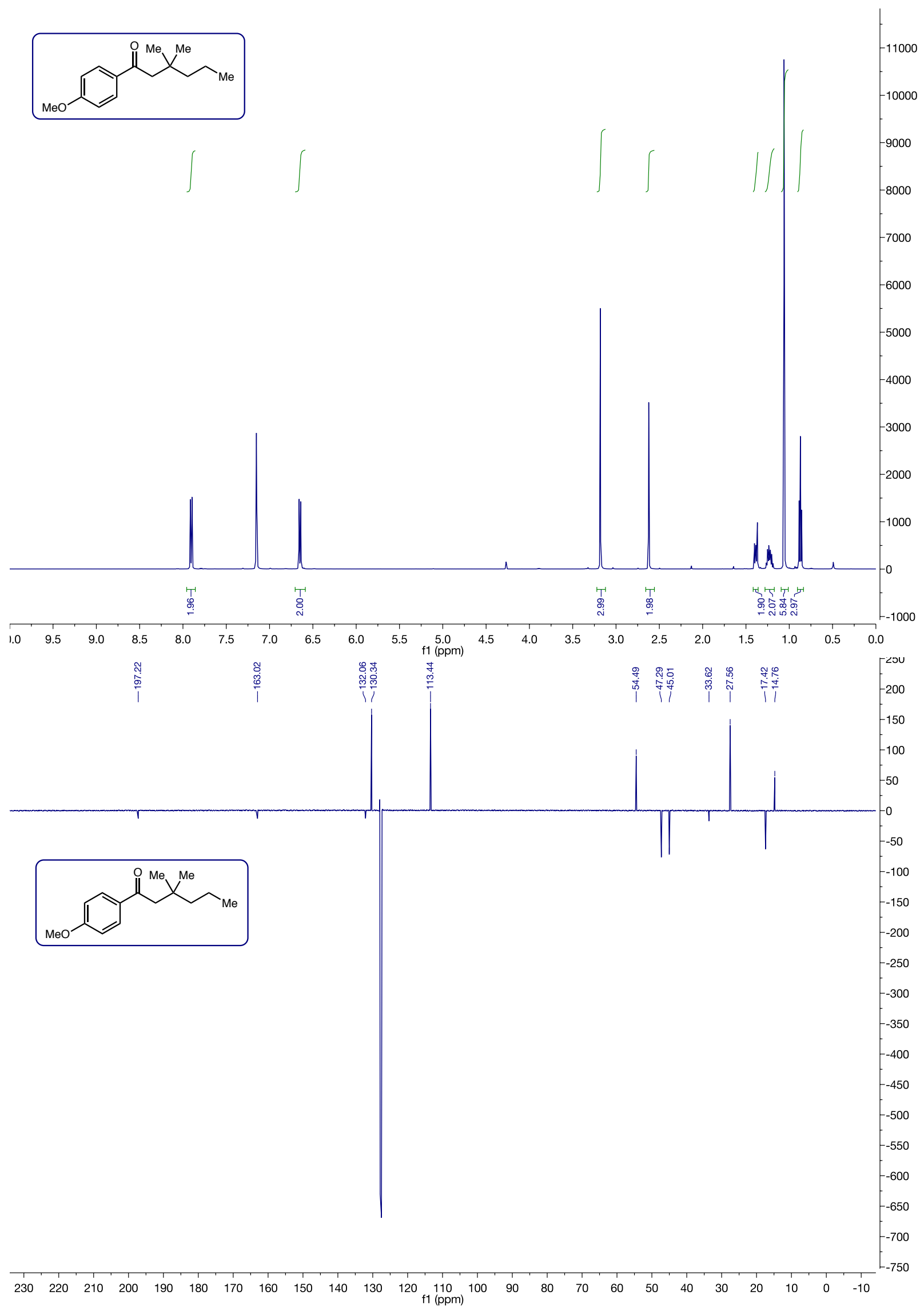




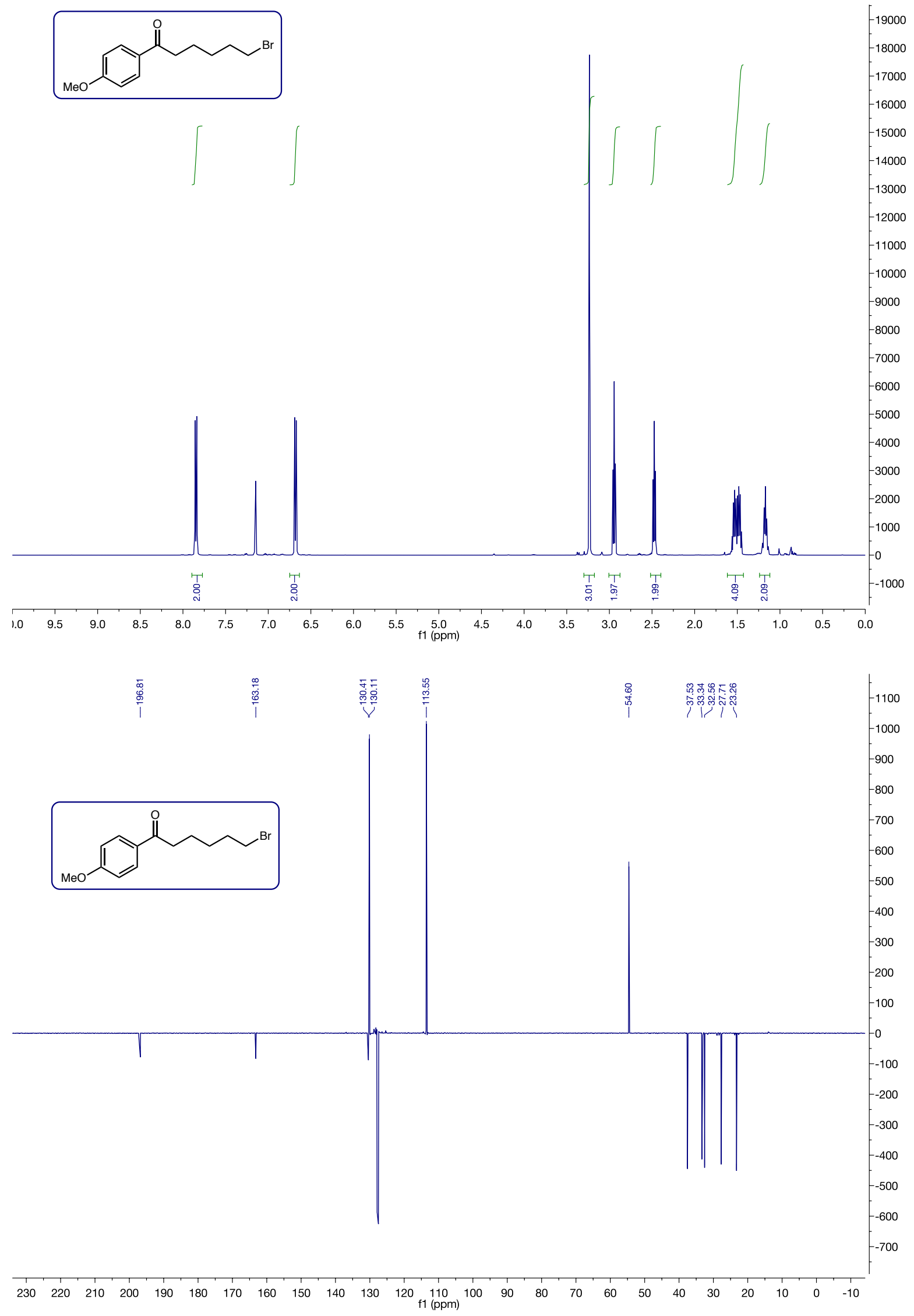




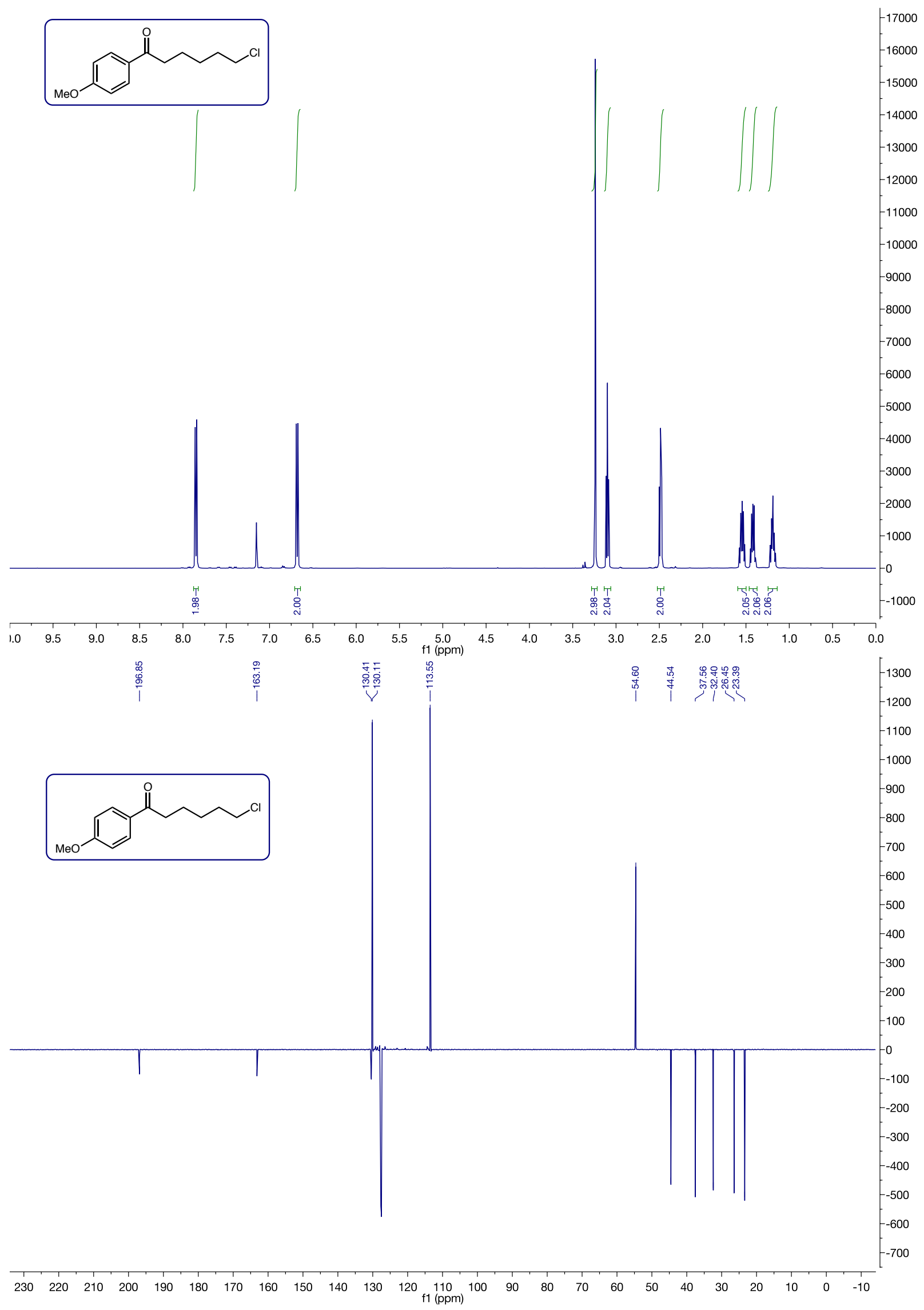




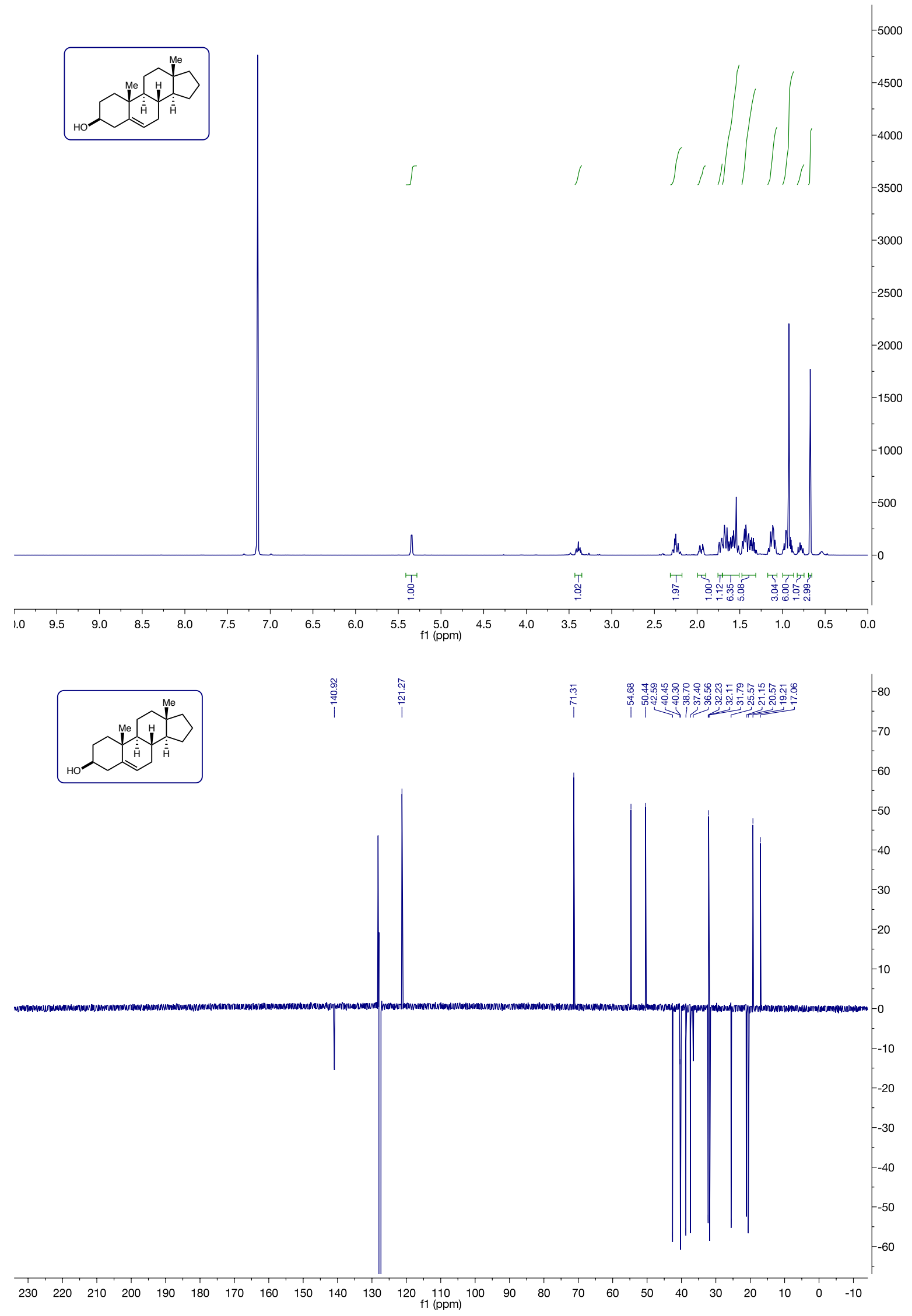



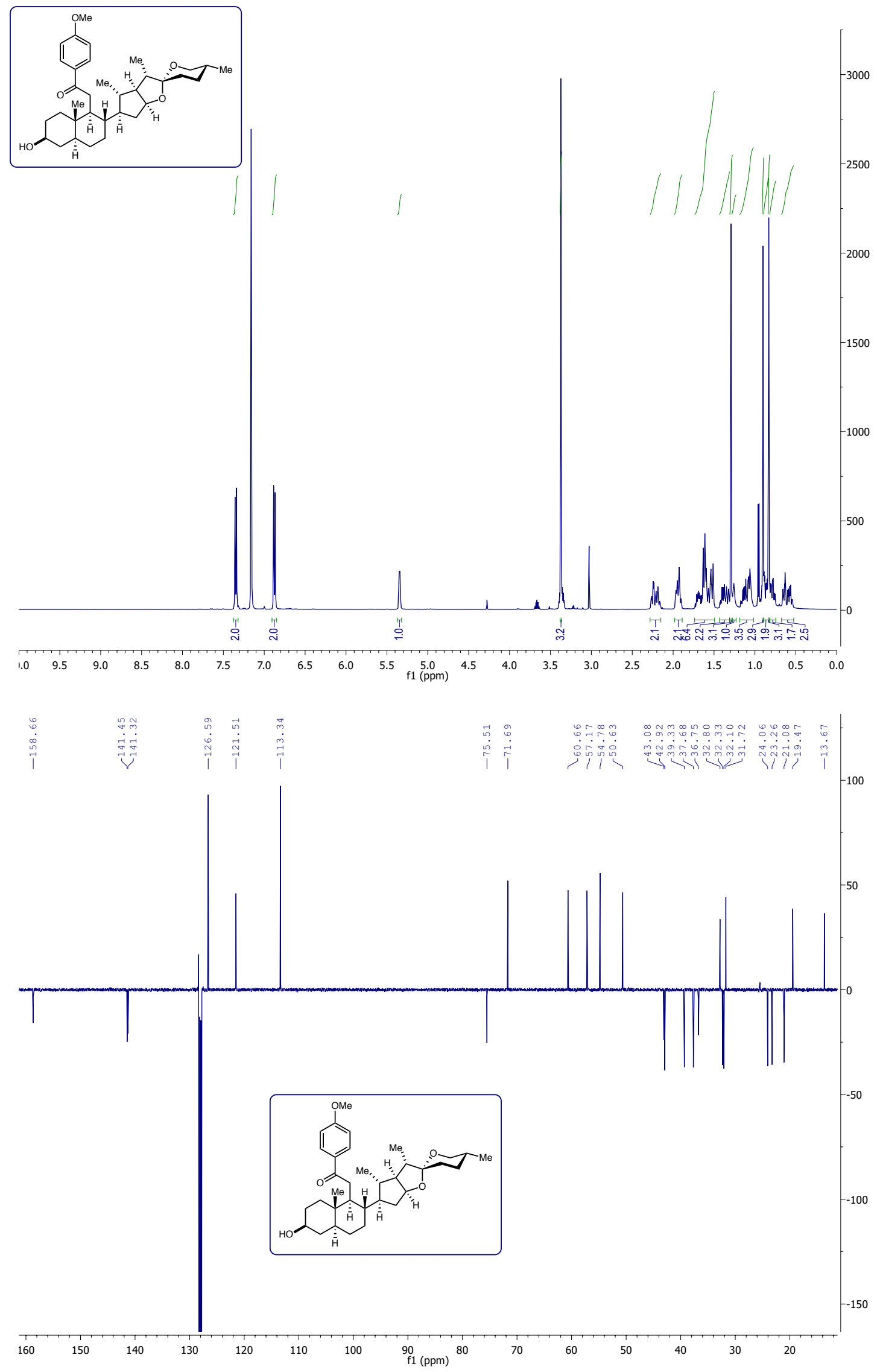


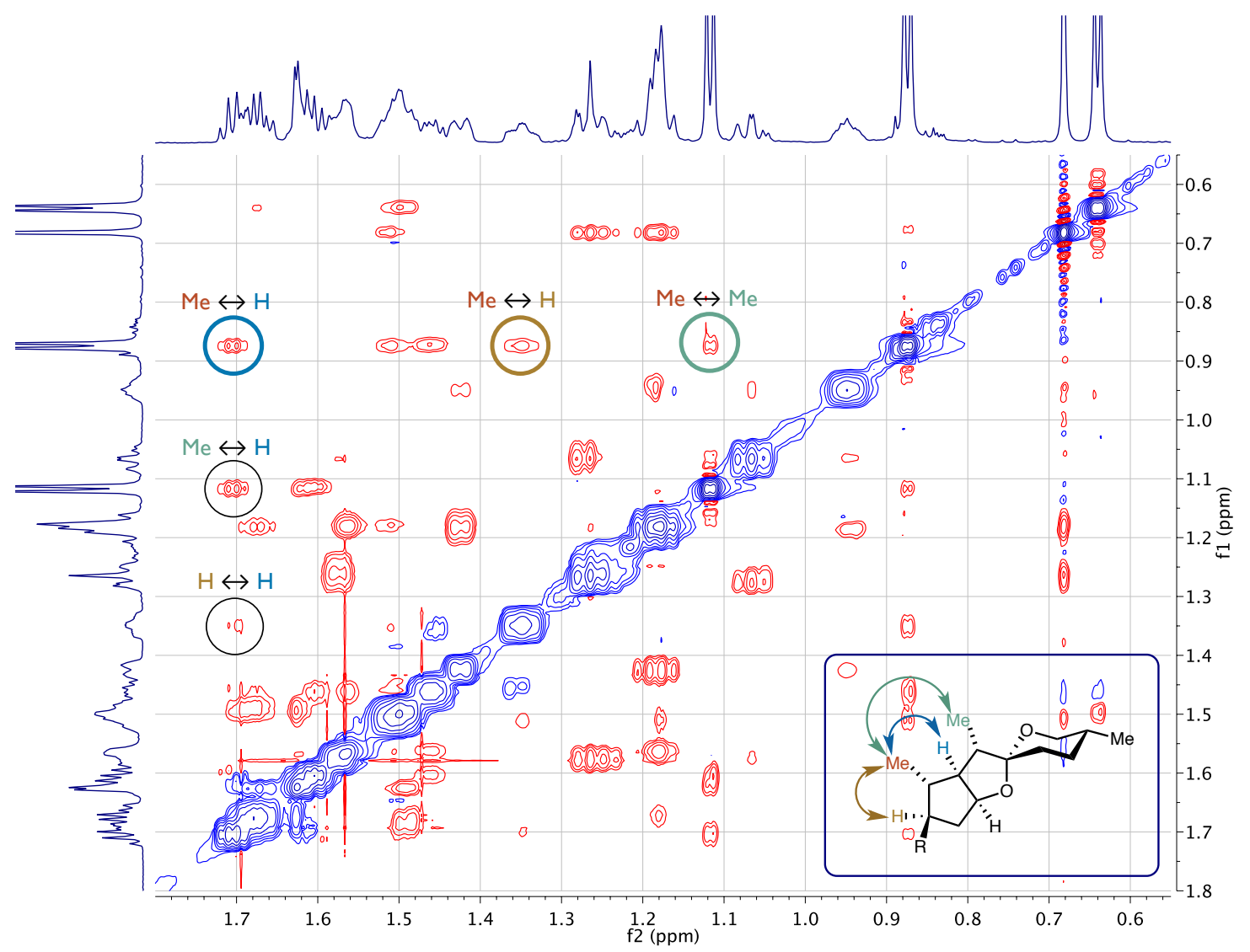

NOESY analysis of the cis-dimethyl product, annotated with important cross-peaks. Firstly, both methyls (Me and $\mathrm{Me}$ ) show nuclear Overhauser effect to the center proton $(\mathrm{H})$ of the same magnitude, signifying their similar spatial distance to $\mathrm{H}$. Secondly, Me is observed to be spatially close to side proton $(\mathrm{H})$, which is on the same face as the center proton H. Lastly, Me and Me also exhibits NOE against each other. This evidence, taken together, was determined to indicate that the two methyl groups in the major isomer are in a cis conformation. 

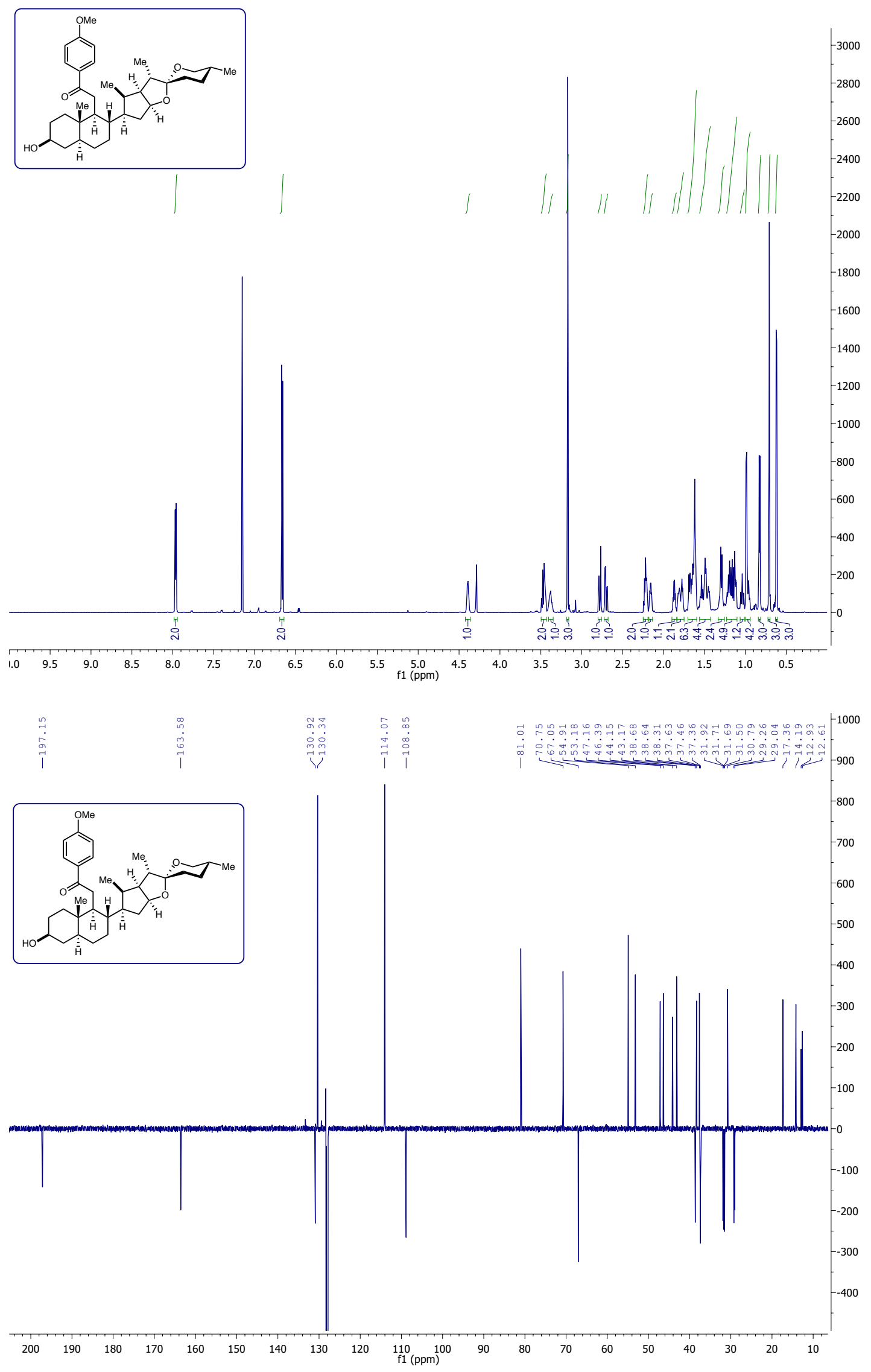


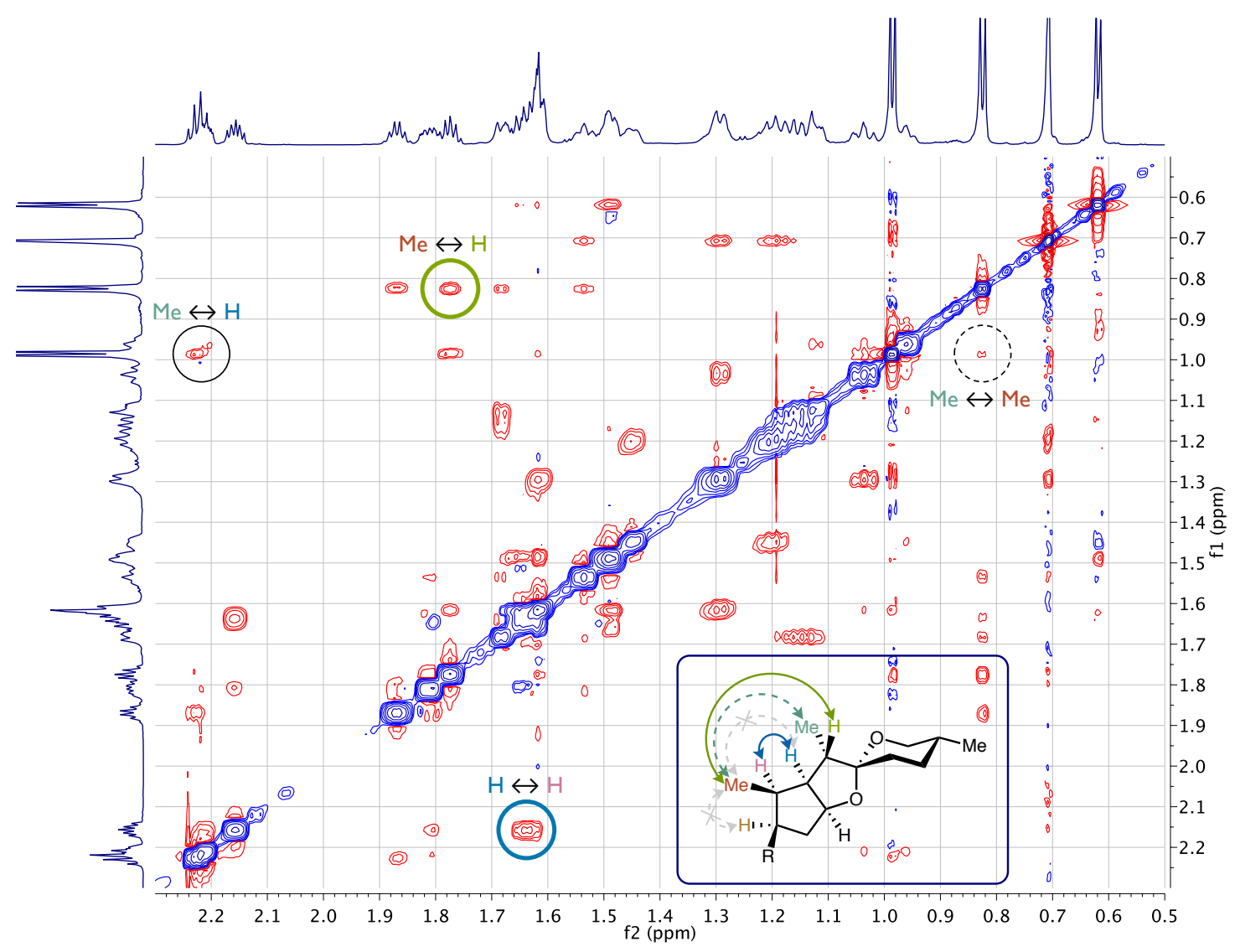

NOESY analysis of the trans-dimethyl product, annotated with important cross-peaks. Firstly, comparing against the NOESY of the cis isomer, it is noted that there is only one methyl, namely the distant $\mathrm{Me}$, in close contact with the center proton $\mathrm{H}$. Secondly, the geminal proton $\mathrm{H}$ of $\mathrm{Me}$ is now proximal to the central H. Thirdly, a significantly weaker NOE is observed between Me and Me. Rather, Me shows strong NOE to the geminal proton $\mathrm{H}$ of the distant Me. This evidence suggests a trans conformation for this minor isomer. 


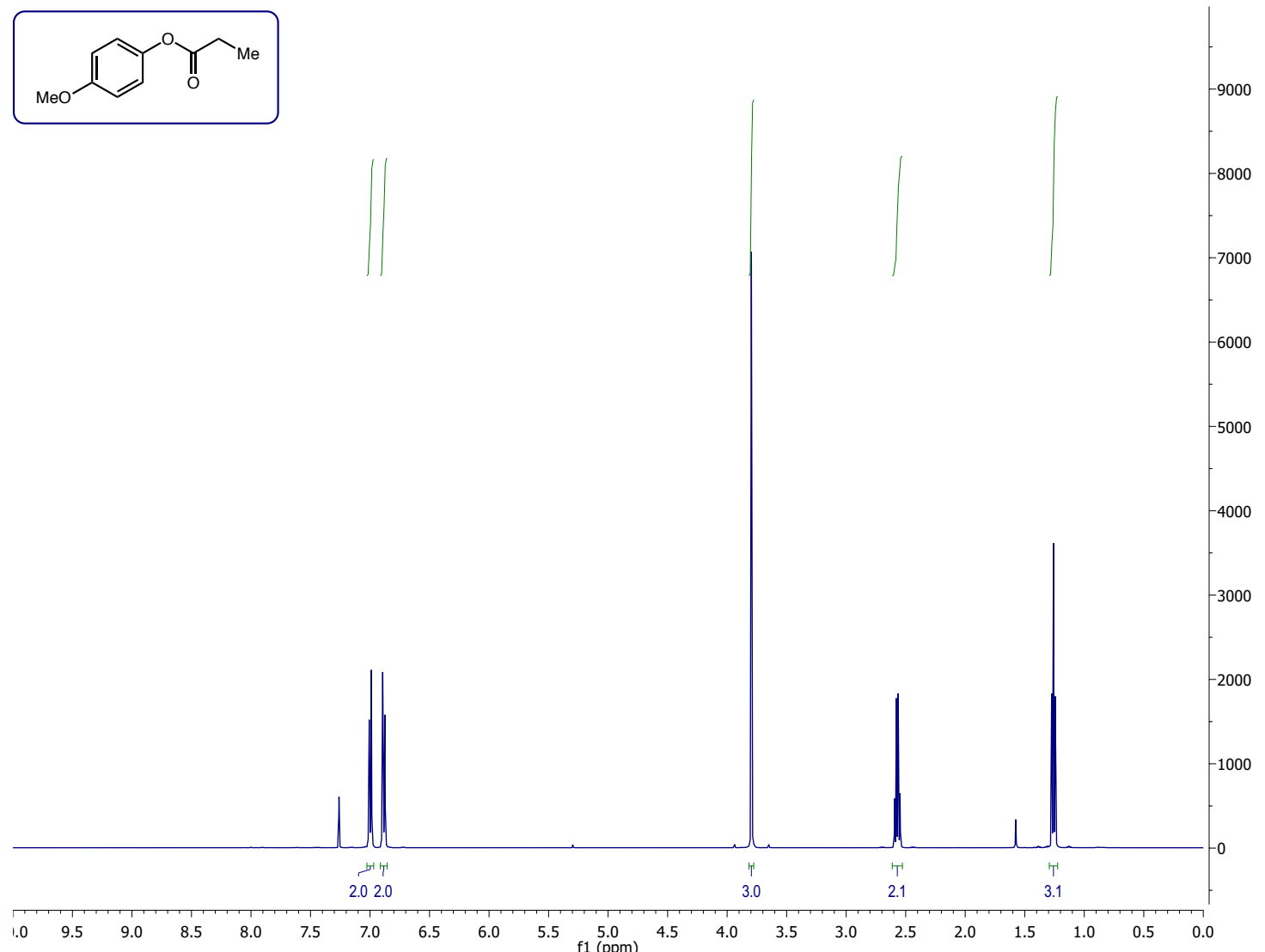




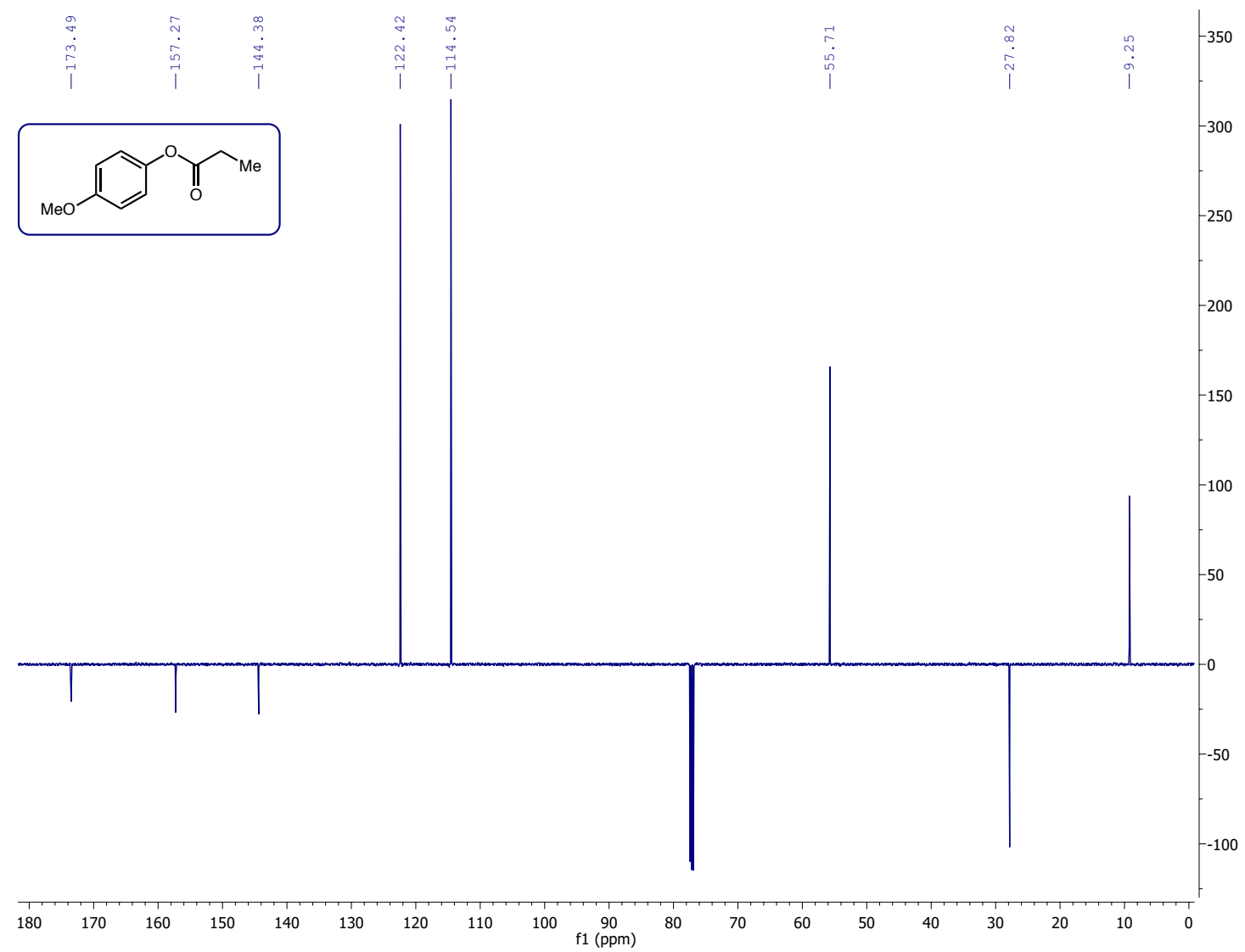




\section{Stern-Volmer Studies}

Stern-Volmer experiments tracking the quenching of the phosphorescence of $\left[\operatorname{Ir}\left(\mathrm{dF}\left(\mathrm{CF}_{3}\right) \text { ppy }\right)_{2}\left(5,5^{\prime} \mathrm{d}\left(\mathrm{CF}_{3}\right)\right.\right.$ bpy $\left.)\right]\left(\mathrm{PF}_{6}\right) \quad(\mathbf{C})$ were conducted on an Agilent Cary Eclipse Fluorescence Spectrophotometer using the Cary Eclipse Scan Application. Fresh stock solutions of Ir photocatalyst $\mathbf{C}$, 1-(4-methoxyphenyl)cyclooctan-1-ol (SM), and collidine were prepared in $\mathrm{CH}_{2} \mathrm{Cl}_{2}$ and mixed together at varying concentrations in volumetric flasks. All liquid transfers were done in the glovebox. The solutions were loaded into quartz cuvettes, sealed under inert atmosphere, and shielded from light exposure before sample collection. The samples were irradiated at $440 \mathrm{~nm}$, and three-scan average emission intensities at $586 \mathrm{~nm}$ were recorded. Each concentration combination was repeated four times, and the final average slope of $\mathrm{I}_{0} / \mathrm{I}$ to concentration is reported here as the $K_{\mathrm{sv}}$. 


\section{Constant [collidine], varied alcohol substrate [SM]}

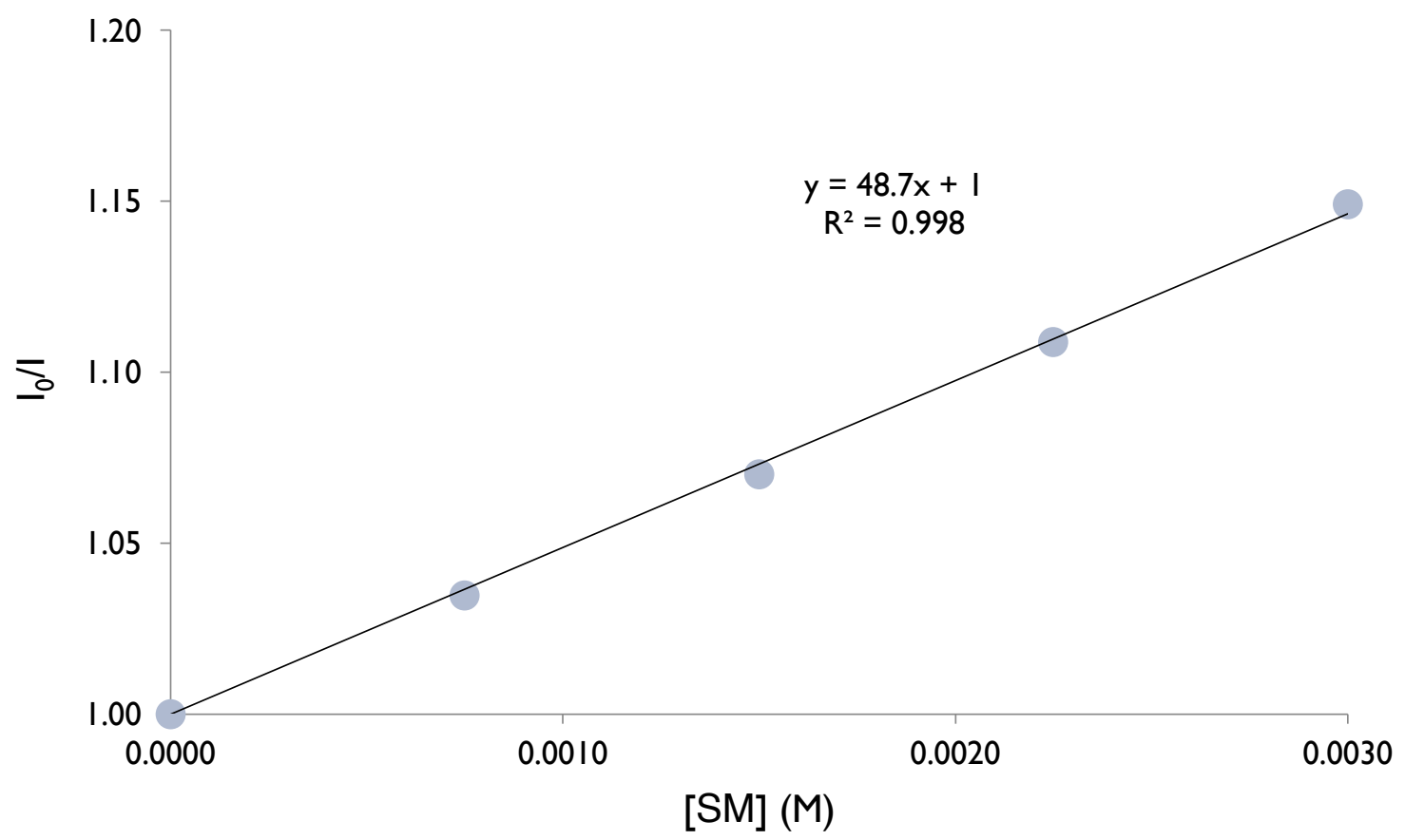

Figure S1. Stern-Volmer plot of $\left[\operatorname{Ir}\left(\mathrm{dF}_{(}\left(\mathrm{CF}_{3}\right) \mathrm{ppy}\right)_{2}\left(5,5^{\prime} \mathrm{d}\left(\mathrm{CF}_{3}\right) \mathrm{bpy}\right)\right]\left(\mathrm{PF}_{6}\right)(244 \mu \mathrm{M})$ with varied $[\mathrm{SM}]$ in the presence of a constant concentration of collidine $(7.22 \mathrm{mM})$ in $\mathrm{CH}_{2} \mathrm{Cl}_{2}$ at $23{ }^{\circ} \mathrm{C}$.

Table S1. Individual results from Stern-Volmer quenching experiments with $\left[\operatorname{Ir}\left(\mathrm{dF}\left(\mathrm{CF}_{3}\right) \text { ppy }\right)_{2}\left(5,5^{\prime} \mathrm{d}\left(\mathrm{CF}_{3}\right) \mathrm{bpy}\right)\right]\left(\mathrm{PF}_{6}\right)(244 \mu \mathrm{M})$ with varied $[\mathrm{SM}]$ in the presence of collidine in $\mathrm{CH}_{2} \mathrm{Cl}_{2}$ at $23{ }^{\circ} \mathrm{C}$.

\begin{tabular}{|c|c|c|c|c|c|c|c|}
\hline \multirow[b]{2}{*}{ [Colldine] (M) } & \multirow[b]{2}{*}{ [SM] (M) } & \multicolumn{4}{|c|}{$I_{\text {emission }}(586 \mathrm{~nm})$} & \multirow[b]{2}{*}{ mean $I_{\text {emission }}$} & \multirow[b]{2}{*}{$\mathrm{I}_{0} / \mathrm{I}$} \\
\hline & & run I & run 2 & run 3 & run 4 & & \\
\hline 7.22E-03 & $0.00 E+00$ & 161.199 & $|61.03|$ & 163.145 & I58.074 & 160.862 & 1.00 \\
\hline $7.22 \mathrm{E}-03$ & $7.50 \mathrm{E}-04$ & 154.695 & 155.840 & 157.740 & 153.645 & 155.480 & 1.03 \\
\hline $7.22 \mathrm{E}-03$ & I.50E-03 & 152.654 & 146.342 & 150.799 & $151.49 \mid$ & $|50.32|$ & 1.07 \\
\hline $7.22 \mathrm{E}-03$ & $2.25 \mathrm{E}-03$ & | 46.802 & 145.864 & 143.602 & 144.056 & $|45.08|$ & I.II \\
\hline $7.22 \mathrm{E}-03$ & $3.00 \mathrm{E}-03$ & |40.62 | & 141.546 & I 40.524 & | 37.333 & 140.006 & 1.15 \\
\hline
\end{tabular}




\section{Constant [SM], varied [collidine]}

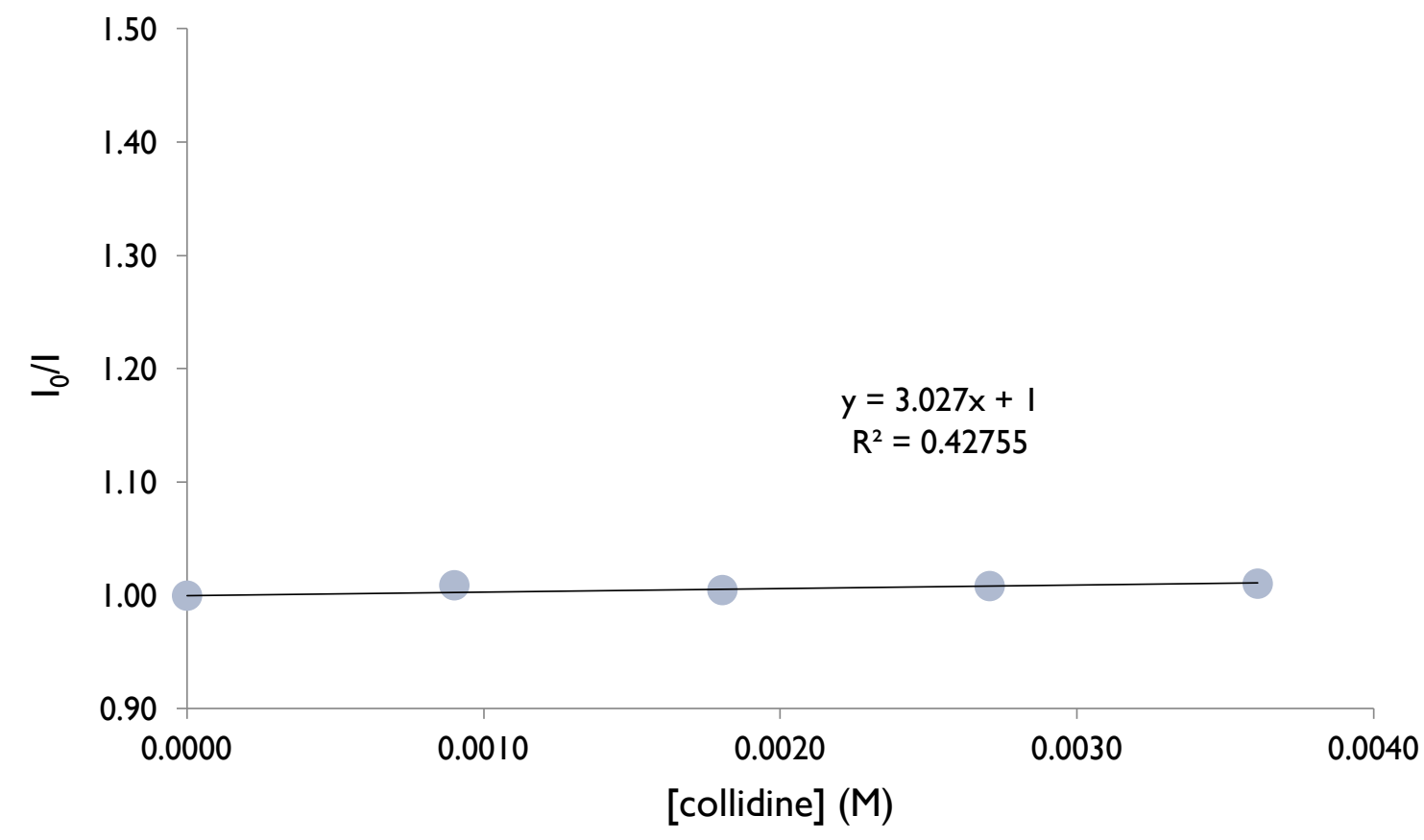

Figure S2. Stern-Volmer plot of $\left.\left[\operatorname{Ir}\left(\mathrm{dF}_{\left(\mathrm{CF}_{3}\right)}\right) \text { ppy }\right)_{2}\left(5,5^{\prime} \mathrm{d}\left(\mathrm{CF}_{3}\right) \mathrm{bpy}\right)\right]\left(\mathrm{PF}_{6}\right)(244 \mu \mathrm{M})$ with varied [collidine] in the presence of a constant concentration of SM $(15.0 \mathrm{mM})$ in $\mathrm{CH}_{2} \mathrm{Cl}_{2}$ at $23{ }^{\circ} \mathrm{C}$.

Table S2. Individual results from Stern-Volmer quenching experiments with $\left[\operatorname{Ir}\left(\mathrm{dF}\left(\mathrm{CF}_{3}\right) \text { ppy }\right)_{2}\left(5,5^{\prime} \mathrm{d}\left(\mathrm{CF}_{3}\right) \mathrm{bpy}\right)\right]\left(\mathrm{PF}_{6}\right)(244 \mu \mathrm{M})$ with varied [collidine] in the presence of SM in $\mathrm{CH}_{2} \mathrm{Cl}_{2}$ at $23{ }^{\circ} \mathrm{C}$.

\begin{tabular}{|c|c|c|c|c|c|c|c|}
\hline \multirow[b]{2}{*}{ [Colldine] (M) } & \multirow[b]{2}{*}{ [SM] (M) } & \multicolumn{4}{|c|}{$I_{\text {emission }}(586 \mathrm{~nm})$} & \multirow[b]{2}{*}{ mean $\mathrm{I}_{\text {emission }}$} & \multirow[b]{2}{*}{$\mathrm{I}_{0} / \mathrm{I}$} \\
\hline & & run I & run 2 & run 3 & run 4 & & \\
\hline $0.00 \mathrm{E}+00$ & I.50E-02 & 104.956 & 99.300 & 98.479 & 100.563 & 100.824 & 1.00 \\
\hline $9.02 \mathrm{E}-04$ & I.50E-02 & 105.590 & 97.163 & 98.756 & 98.281 & 99.948 & 1.01 \\
\hline I.80E-03 & I.50E-02 & 104.299 & 98.604 & 98.969 & 99.683 & 100.389 & 1.00 \\
\hline 2.7IE-03 & I.50E-02 & 104.126 & 97.898 & 98.404 & 99.683 & 100.028 & 1.01 \\
\hline $3.6 I E-03$ & I.50E-02 & 108.776 & 93.766 & 97.250 & 99.457 & 99.812 & 1.01 \\
\hline
\end{tabular}




\section{Experimental Details for Investigating Long-range PCET (Figure 3)}

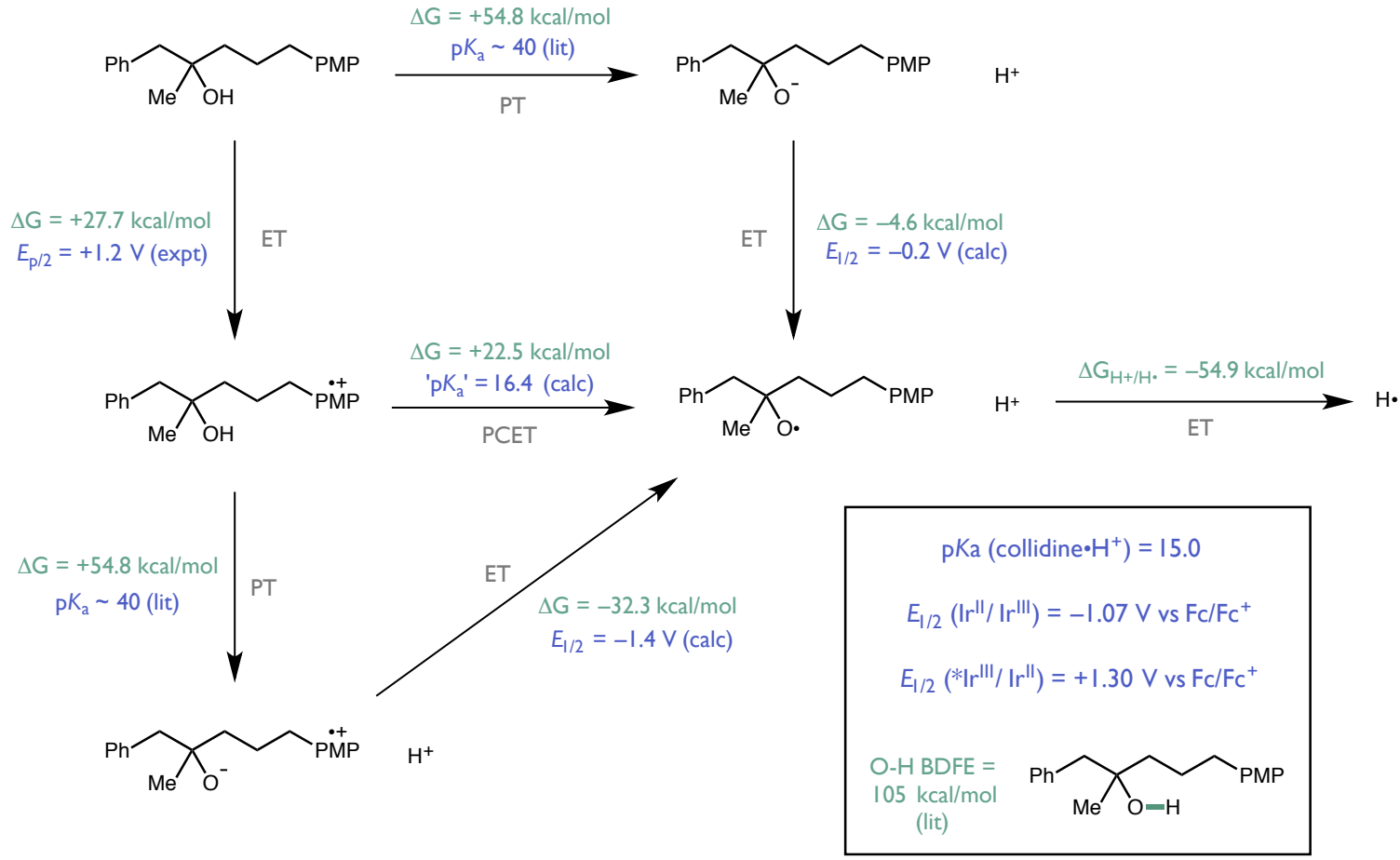

- All values given are for $\mathrm{MeCN}$. All potentials are referenced to $\mathrm{Fc} / \mathrm{Fc}^{+}$.

- Values labelled (calc) are calulated from the thermochemical data presented in the figure using the following expression:

$\operatorname{BDFE}(\mathrm{kcal} / \mathrm{mol})=1.37 \mathrm{pKa}+23.06 \mathrm{E}^{0}+54.9 \mathrm{kcal} / \mathrm{mol}(\mathrm{RT}$ in MeCN$)$

Figure S3. Relationship and energy of different species and mechanistic pathways.

Screw-cap 2-dram vials outfitted with PTFE/silicone septa were charged with the relevant alcohol (0.05 mmol, 1.0 equiv), redistilled collidine $(0.15 \mathrm{mmol}, 3.0$ equiv, $20 \mu \mathrm{L})$, and redistilled thiophenol $(13 \mu \mathrm{mol}, 0.25$ equiv, $1.3 \mu \mathrm{L})$. A $1-\mathrm{mM}$ stock solution of $\left[\operatorname{Ir}\left(\mathrm{dF}\left(\mathrm{CF}_{3}\right) \text { ppy }\right)_{2}\left(5,5^{\prime} \mathrm{d}\left(\mathrm{CF}_{3}\right)\right.\right.$ bpy $\left.)\right]\left(\mathrm{PF}_{6}\right)$ in degassed anhydrous $\mathrm{CH}_{2} \mathrm{Cl}_{2}$ was made, and $0.5 \mathrm{~mL}$ of this solution $(0.5 \mu \mathrm{mol}, 0.01$ equiv in photocatalyst) was added to each vial via syringe. Thereafter, the vials were frozen in liquid nitrogen, evacuated, and let thaw in warm water bath; this process was repeated three times. The reactions were irradiated by strips of blue LED lights set inside a recrystallization dish, and let stir under a nitrogen atmosphere at room temperature with a fan to cool the setup. After 16-18 hours, the irradiation was stopped, and dibenzyl ether $(0.05 \mathrm{mmol}, 1.0$ equiv, $9.5 \mu \mathrm{L})$ was introduced into each reaction as an internal standard. The crude reactions were concentrated, dissolved in benzene- $d_{6}$, and submitted for ${ }^{1} \mathrm{H}$ NMR. Percentage yields are calculated from integral ratio of $\mathrm{ArCOCH}_{2}$ peak to $\left(\mathrm{PhCH}_{2}\right)_{2} \mathrm{O}$ peak. These yields were also confirmed by GC assays developed for the ketone products. 


$$
\text { I sp }{ }^{3} \text { C-atom spacer }
$$
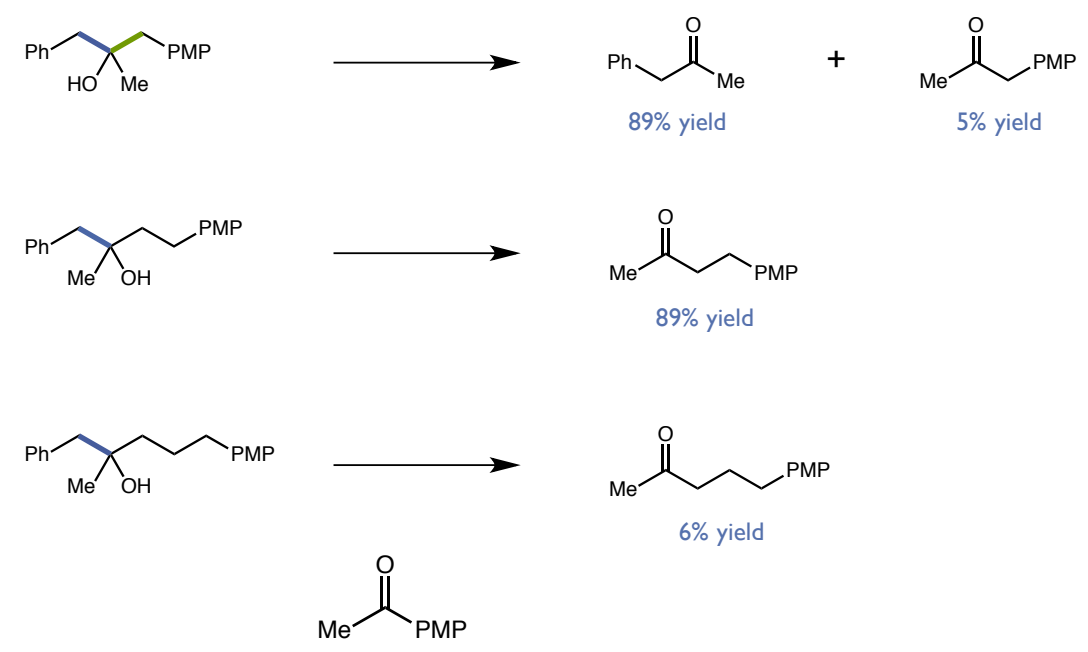

\section{1-(4-methoxyphenyl)ethan-1-one}

Spectra are consistent with reported literature values ${ }^{13}$ and commercial samples.

\section{1-phenylpropan-2-one}

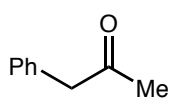

Spectra are consistent with reported literature values. ${ }^{14}$

\section{1-(4-methoxyphenyl)propan-2-one}

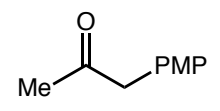

Spectra are consistent with reported literature values ${ }^{15}$ and commercial samples.

\section{4-(4-methoxyphenyl)butan-2-one}

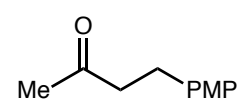

Spectra are consistent with reported literature values ${ }^{16}$ and commercial samples.

\section{5-(4-methoxyphenyl)pentan-2-one}

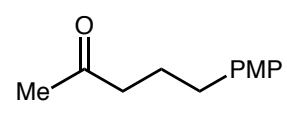

Spectra are consistent with reported literature values. ${ }^{17}$ 


\section{Experimental Details for Investigating the Relationship Between Effective BDFE and Reaction Outcomes (Figure 4)}

Setup for Brønsted bases 2-methoxypyridine, pyridine, and tetrabutylammonium trifluoroacetate followed the procedures outlined in the previous section. For TBA ${ }^{+} \mathrm{TFA}^{-}$, only 1 equiv $(0.05 \mathrm{mmol})$ was used, and the weighing was carried out in a glovebox. Dibenzyl ether $(0.05 \mathrm{mmol}, 1.0$ equiv, $9.5 \mu \mathrm{L})$ was used as an internal standard to calculate percentage yields, based on the ${ }^{1} \mathrm{H}$ NMR signal integral ratio of $\mathrm{ArCOCH}_{2}$ peak to $\left(\mathrm{PhCH}_{2}\right)_{2} \mathrm{O}$ peak. Average isolated yields of 1-mmol reactions are reported for collidine reactions.

\begin{tabular}{|c|c|c|c|c|c|c|c|c|c|c|}
\hline Base & $E_{p / 2}(\mathrm{~V})$ & 0.39 & 0.69 & 0.92 & 0.96 & 1.18 & 1.22 & 1.22 & 1.24 & 1.27 \\
\hline 2-MeO-pyridine & 'BDFE' & 77 & 84 & 90 & 91 & 96 & 97 & 97 & 97 & 98 \\
\hline $\mathrm{p} K_{\mathrm{a}}=9.9$ & Yield (\%) & 0 & 0 & 0 & 0 & 0 & 0 & 0 & $<5$ & 8 \\
\hline pyridine & 'BDFE' & 81 & 88 & 93 & 94 & 99 & 100 & 100 & 101 & 101 \\
\hline $\mathrm{pK} K_{\mathrm{a}}=12.5$ & Yield (\%) & 0 & 0 & 0 & $<5$ & 6 & 16 & 14 & 5 & 19 \\
\hline $\mathrm{CF}_{3} \mathrm{COO}^{-}$ & 'BDFE' & 81 & 88 & 93 & 94 & 99 & 100 & 100 & 101 & 101 \\
\hline $\mathrm{p} K_{\mathrm{a}}=12.5$ & Yield (\%) & 0 & 0 & 0 & 0 & 23 & 87 & 79 & 97 & 18 \\
\hline collidine & 'BDFE' & 84 & 91 & 97 & 98 & 103 & 104 & 104 & 104 & 105 \\
\hline $\mathrm{p} K_{\mathrm{a}}=15$ & Yield (\%) & 0 & 0 & $<5$ & 7 & 86 & 86 & 82 & 41 & 84 \\
\hline
\end{tabular}

Figure S4. Effective BDFE and reaction outcome. 


\section{Cyclic Voltammograms of Starting Materials}

All voltammograms were taken at room temperature using a saturated calomel (SCE) reference electrode, a mesh platinum $(\mathrm{Pt})$ counter electrode, and a glassy carbon working electrode. The conditions of the experiments were the following: an acetonitrile solution of 100 $\mathrm{mM}$ tetrabutylammonium hexafluorophosphate $\left(\mathrm{NBu}_{4} \mathrm{PF}_{6}\right)$ and $1 \mathrm{mM}$ aryl alcohol, a scan rate of $0.1 \mathrm{~V} / \mathrm{s}$, and a positive initial scan direction. The reported potentials were averages over segments, and were taken at half-height of the cathodic peaks $\left(E_{\mathrm{p} / 2}\right)$ of the compounds, since all oxidations were nonreversible. To convert the potentials from SCE to $\mathrm{Fc} / \mathrm{Fc}^{+}$reference, $380 \mathrm{mV}$ were subtracted from the measured values. The positive peaks on the return sweep of most substrates were thought to signify an ECE-type mechanism. ${ }^{18}$

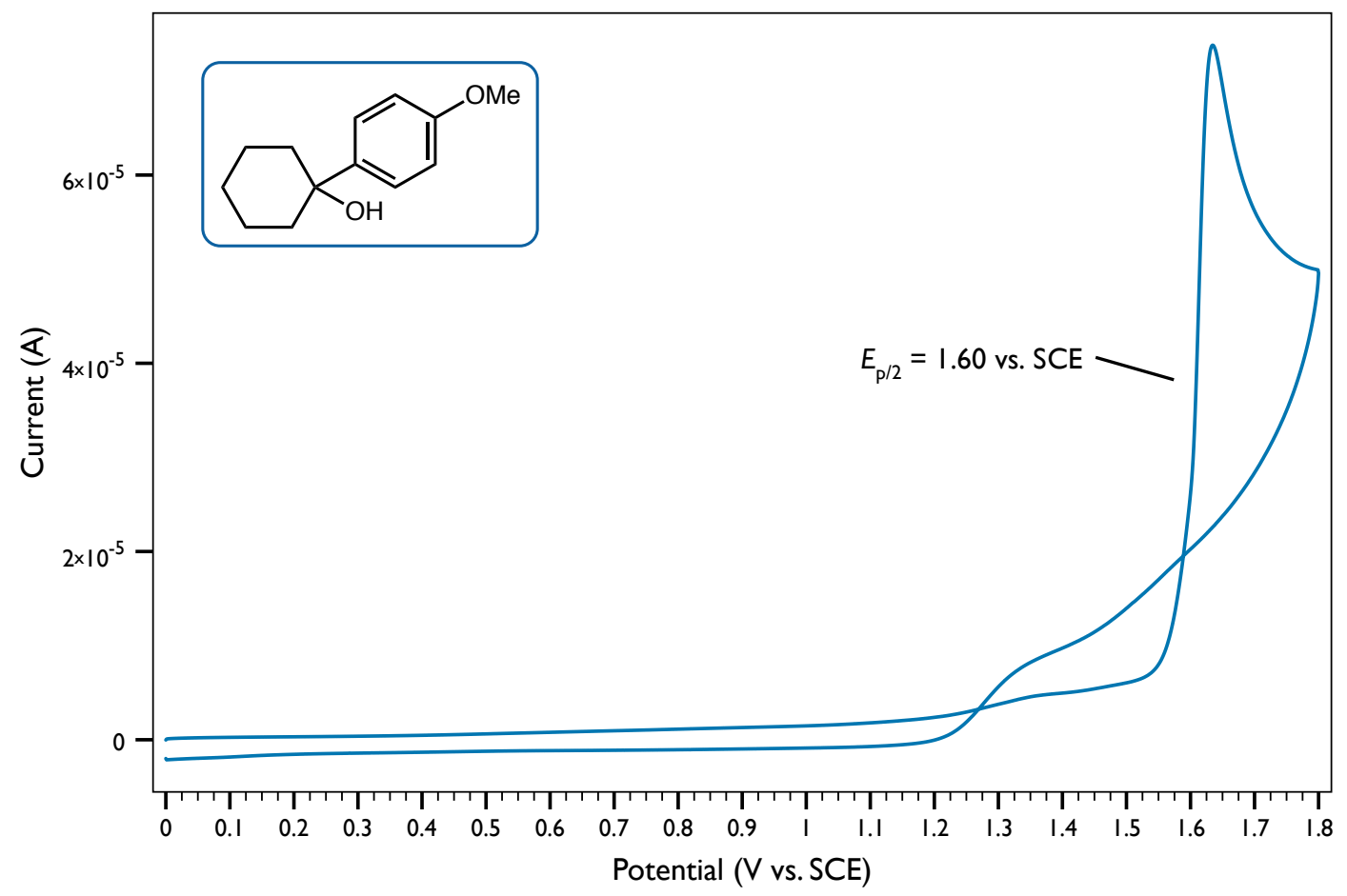

Figure S5. CV of 1-(4-methoxyphenyl)cyclohexan-1-ol in MeCN 


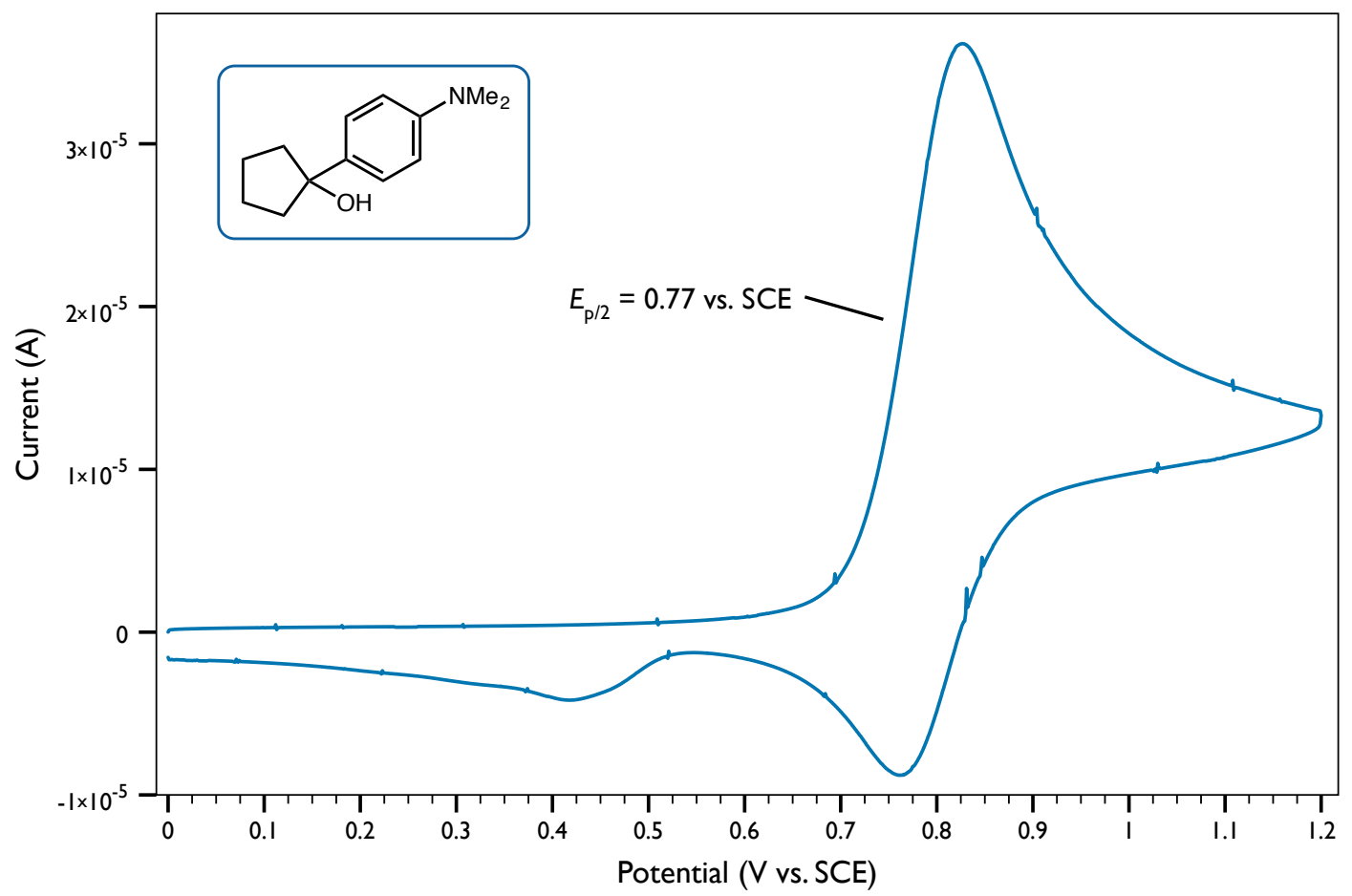

Figure S6. CV of 1-(4-(dimethylamino)phenyl)cyclopentanol in $\mathrm{MeCN}$

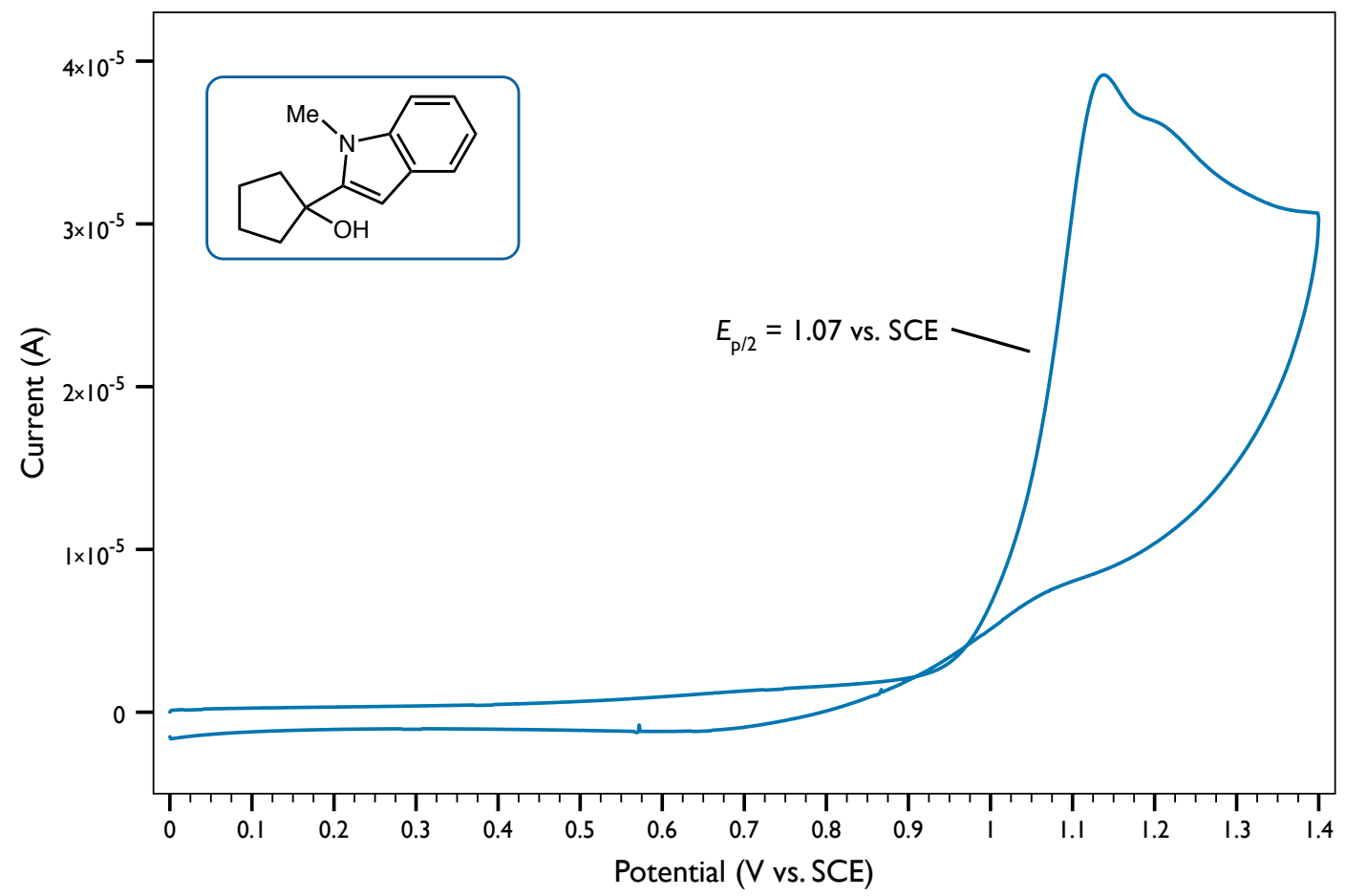

Figure S7. CV of 1-(1-methyl-1H-indol-2-yl)cyclopentanol in MeCN 


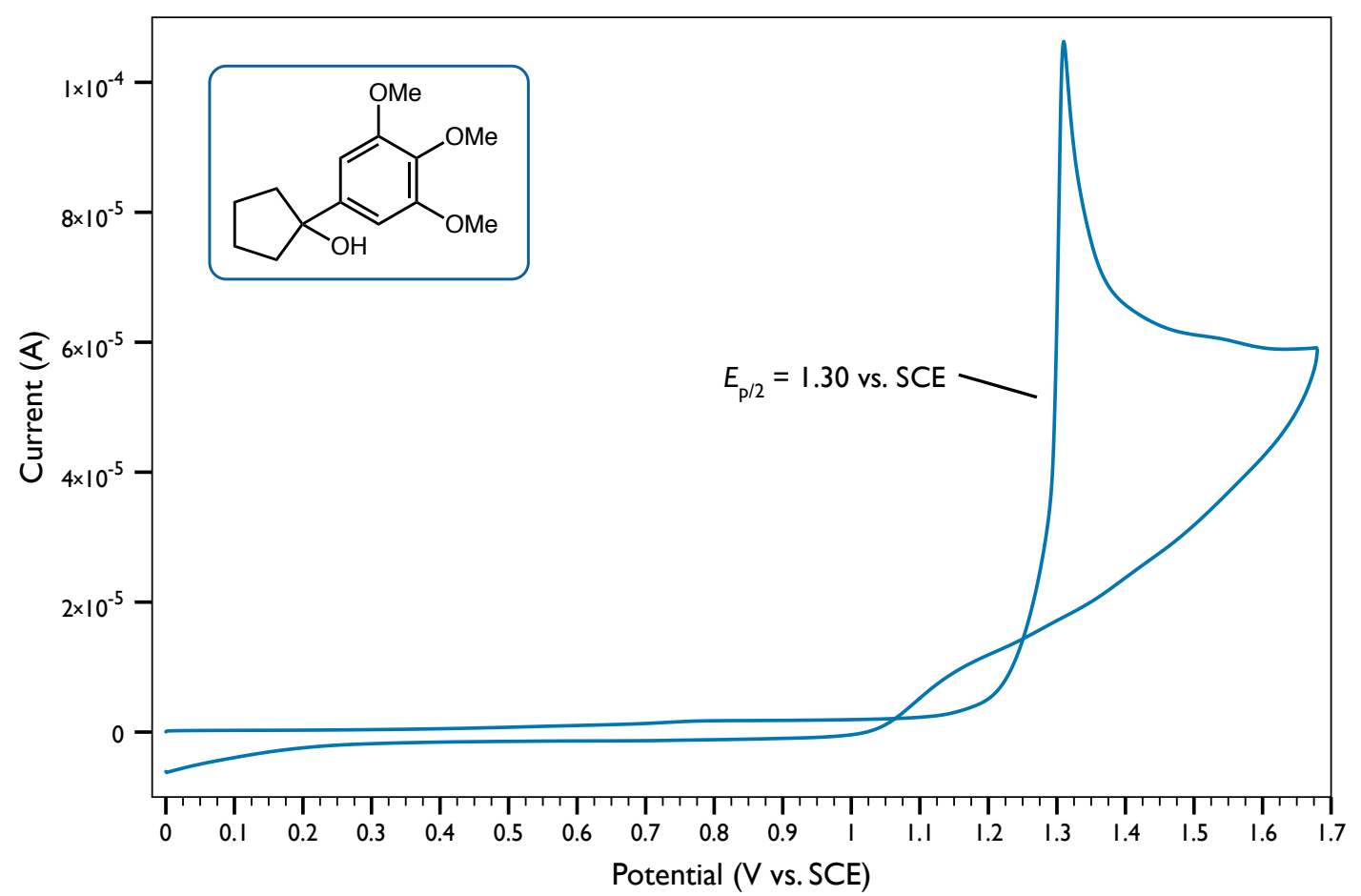

Figure S8. CV of 1-(3,4,5-trimethoxyphenyl)cyclopentan-1-ol in MeCN

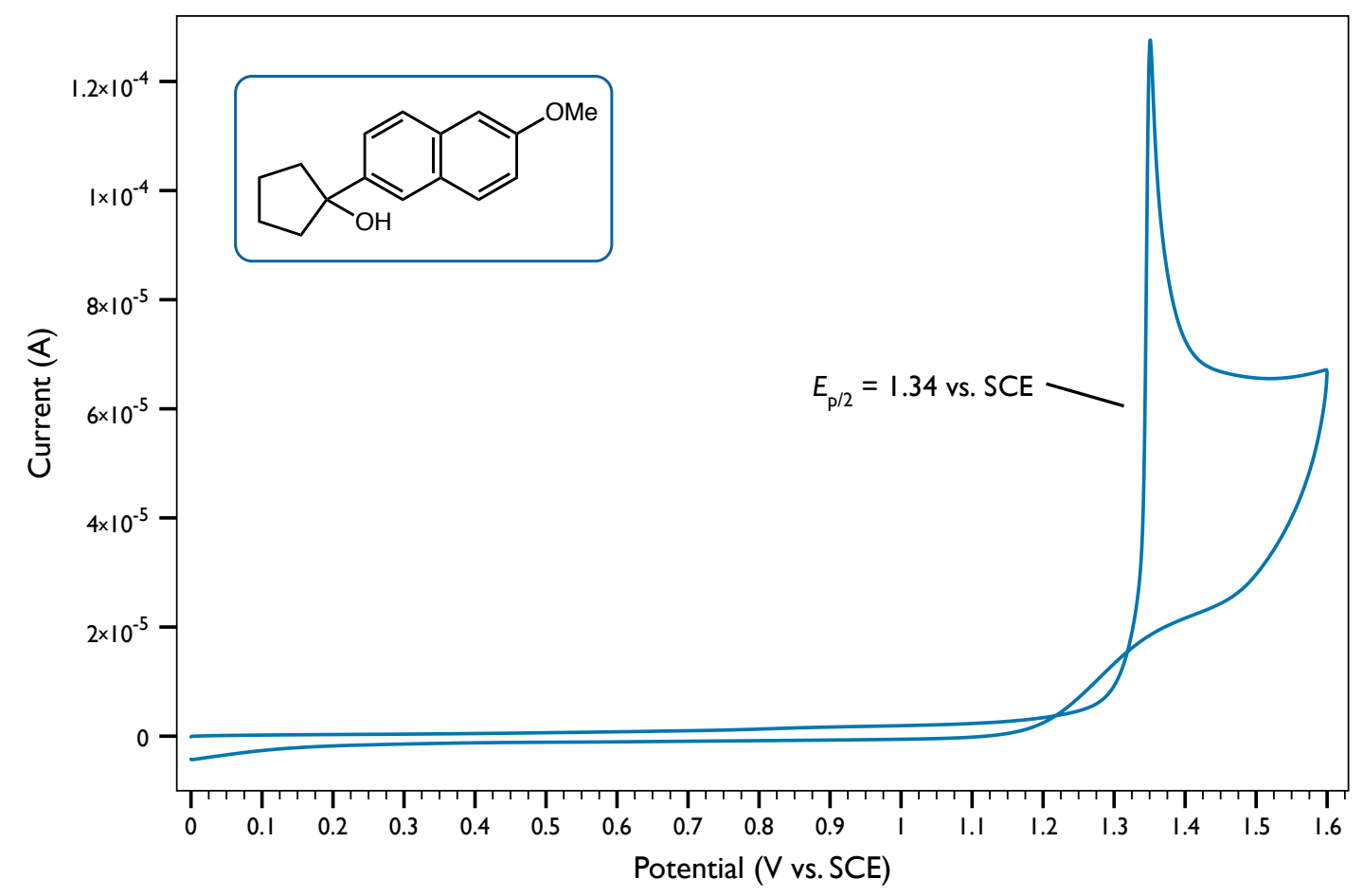

Figure S9. CV of 1-(6-methoxynaphthalen-2-yl)cyclopentan-1-ol in MeCN 


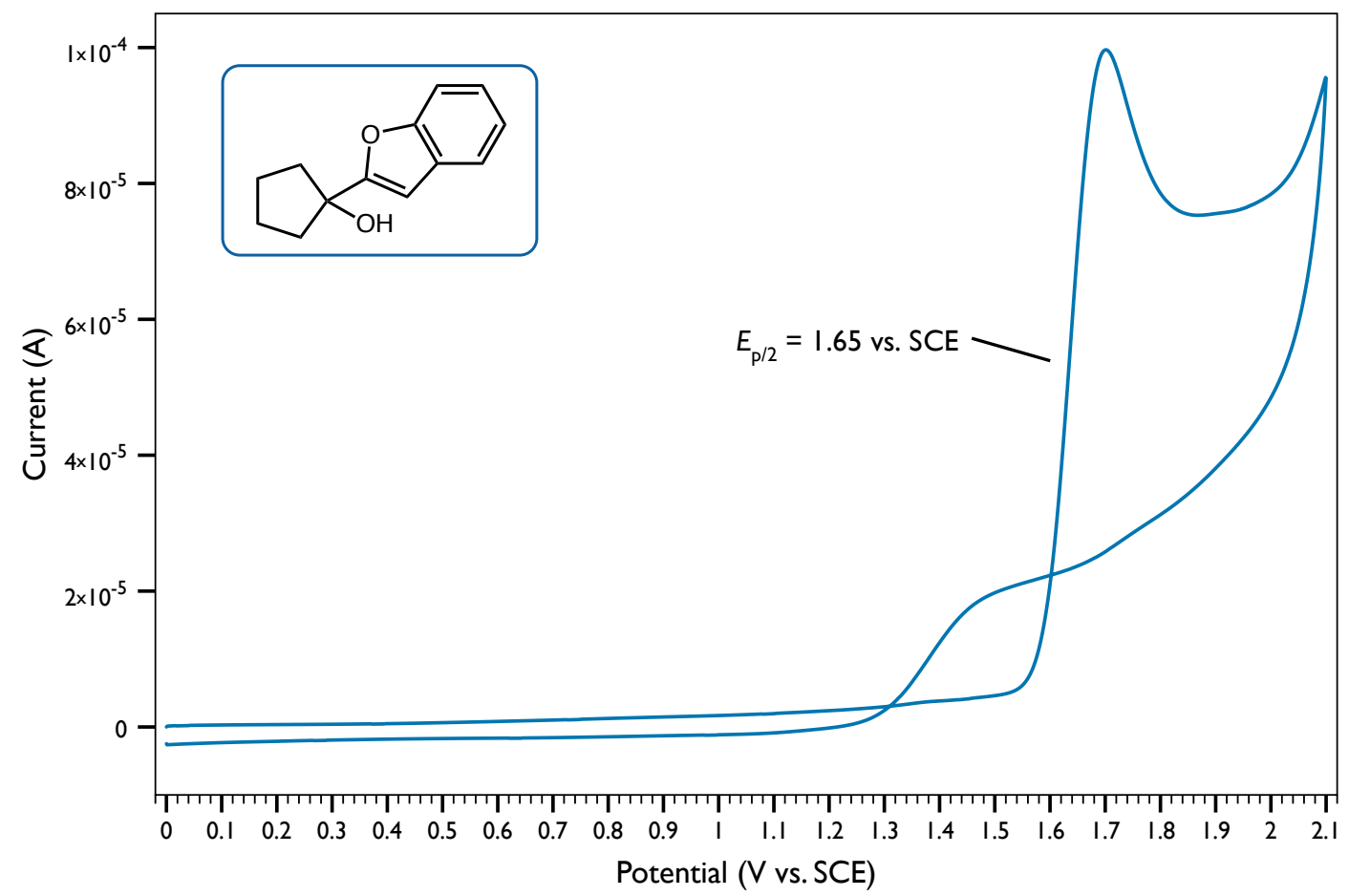

Figure S10. CV of 1-(benzofuran-2-yl)cyclopentanol in $\mathrm{MeCN}$

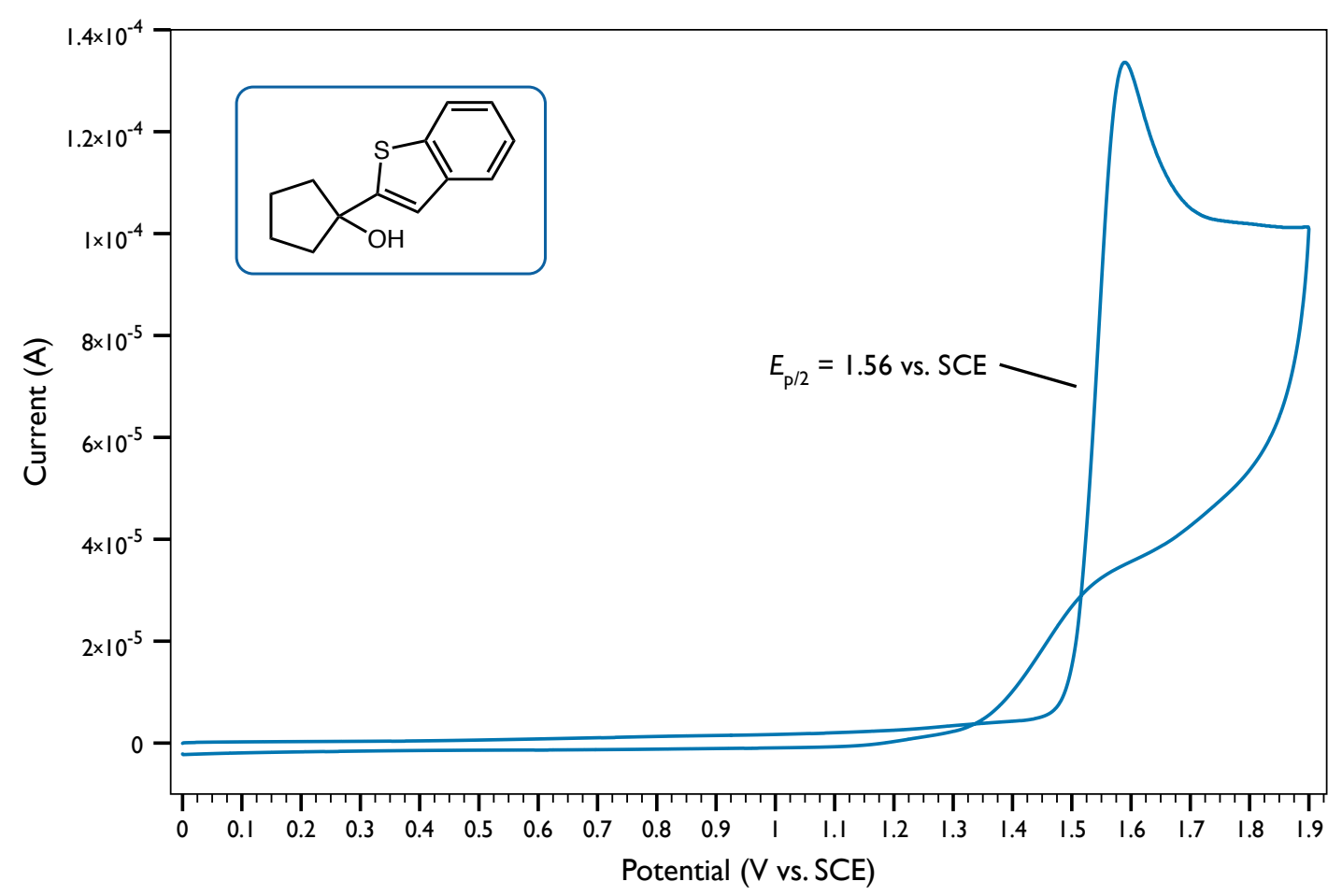

Figure S11. CV of 1-(benzo[b]thiophen-2-yl)cyclopentanol in MeCN 


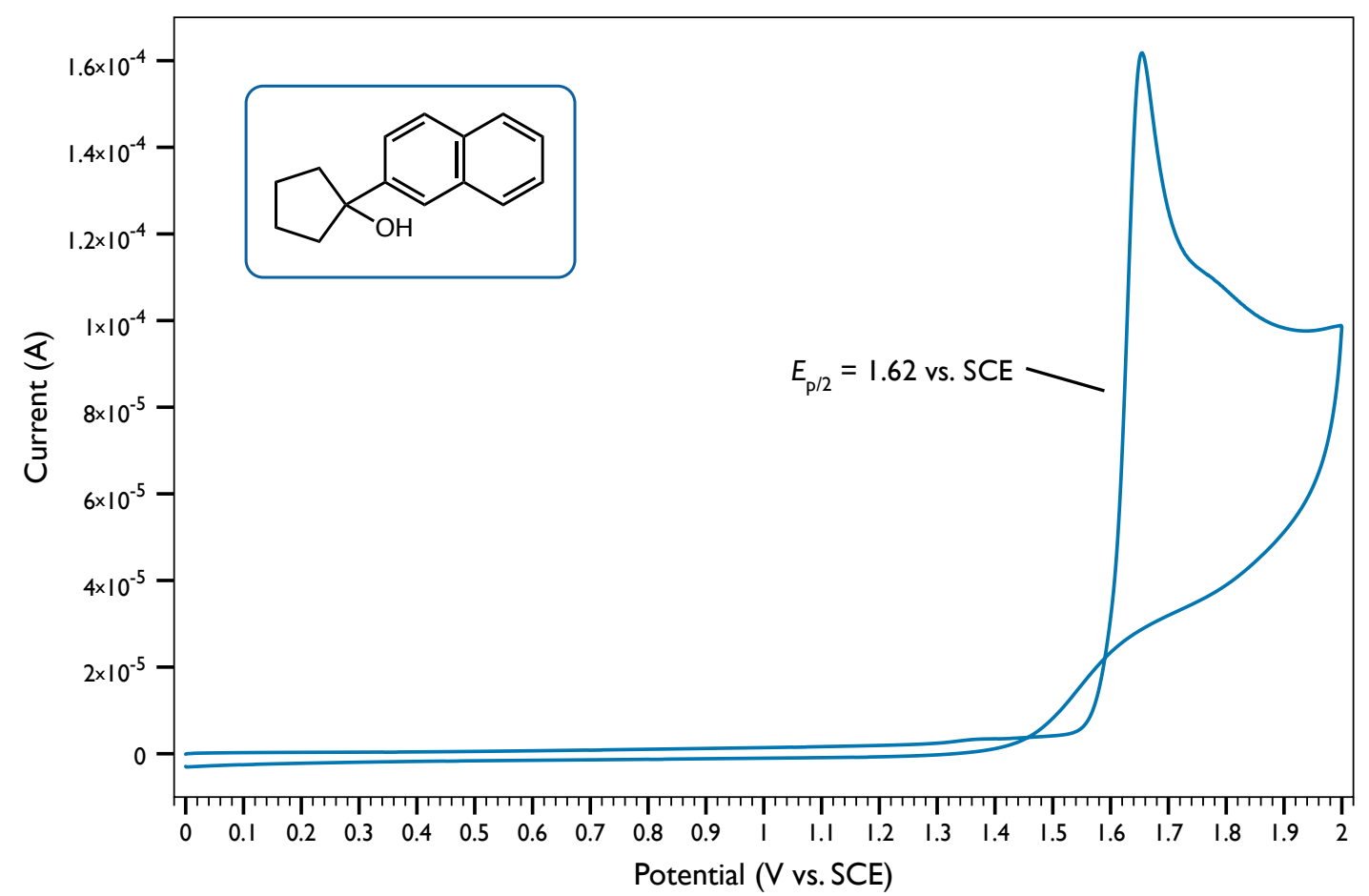

Figure S12. CV of 1-(naphthalen-2-yl)cyclopentanol in $\mathrm{MeCN}$

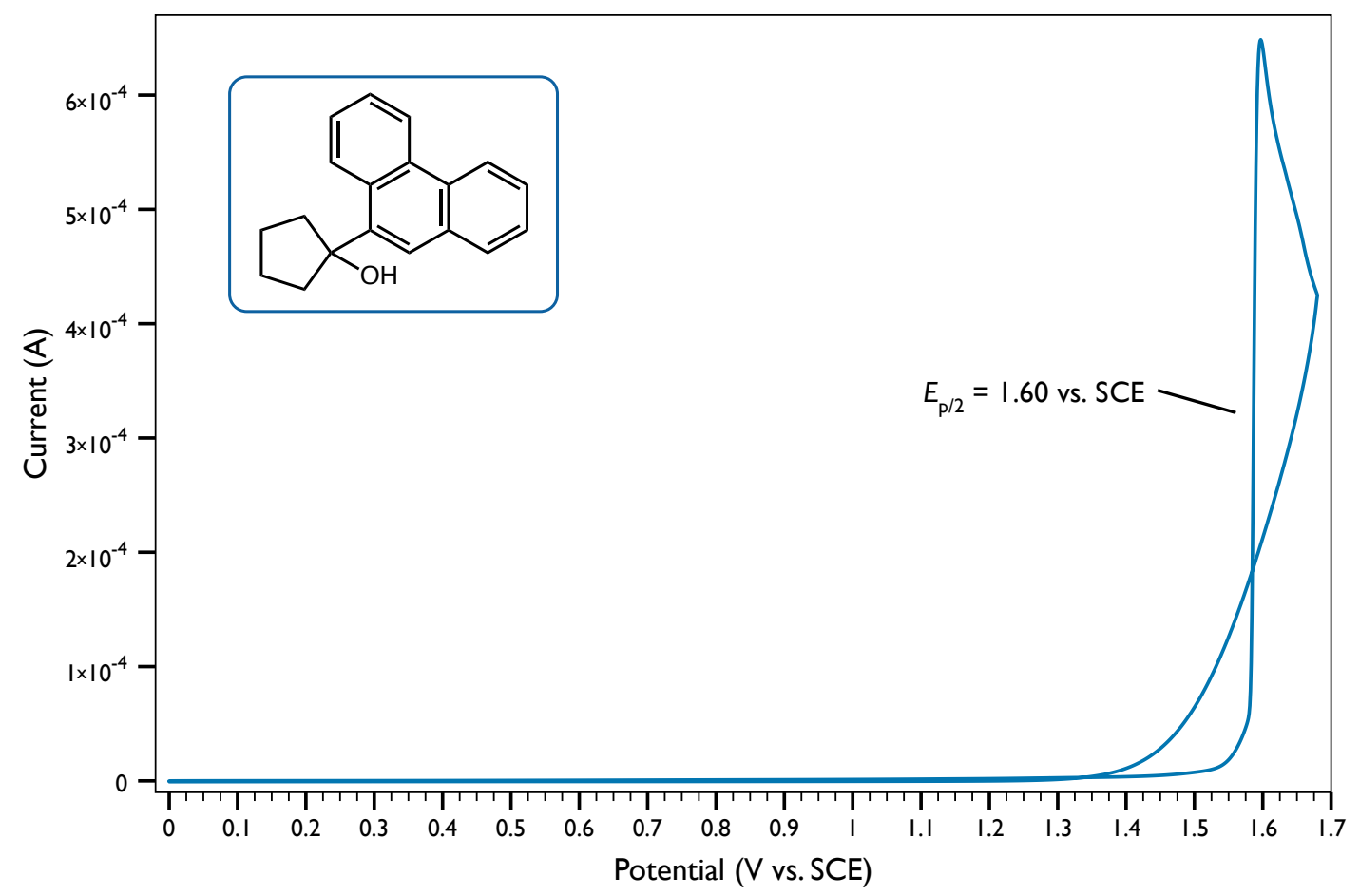

Figure S13. CV of 1-(phenanthren-9-yl)cyclopentanol in $\mathrm{MeCN}$ 


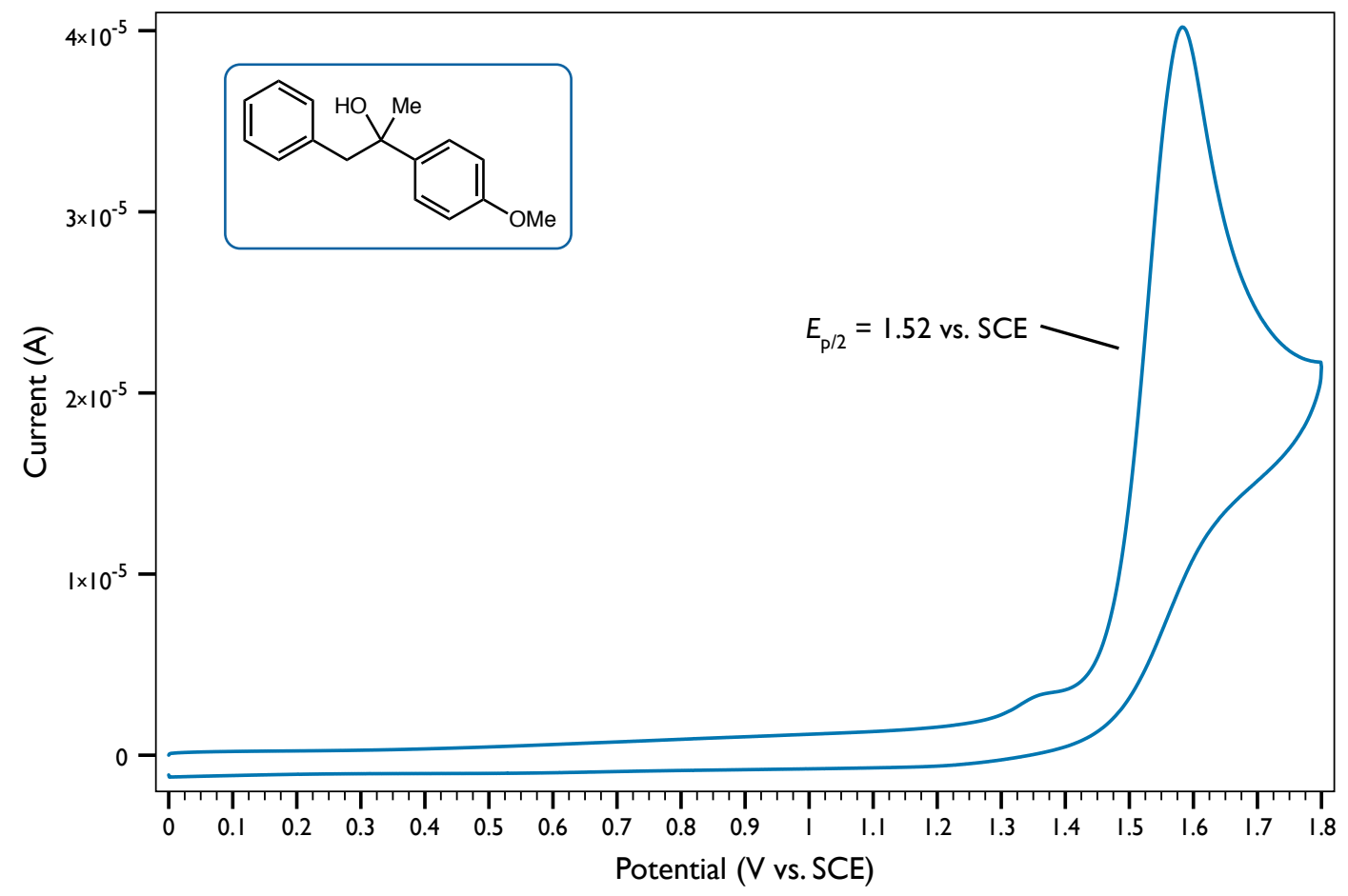

Figure S14. CV of 2-(4-methoxyphenyl)-1-phenylpropan-2-ol in MeCN

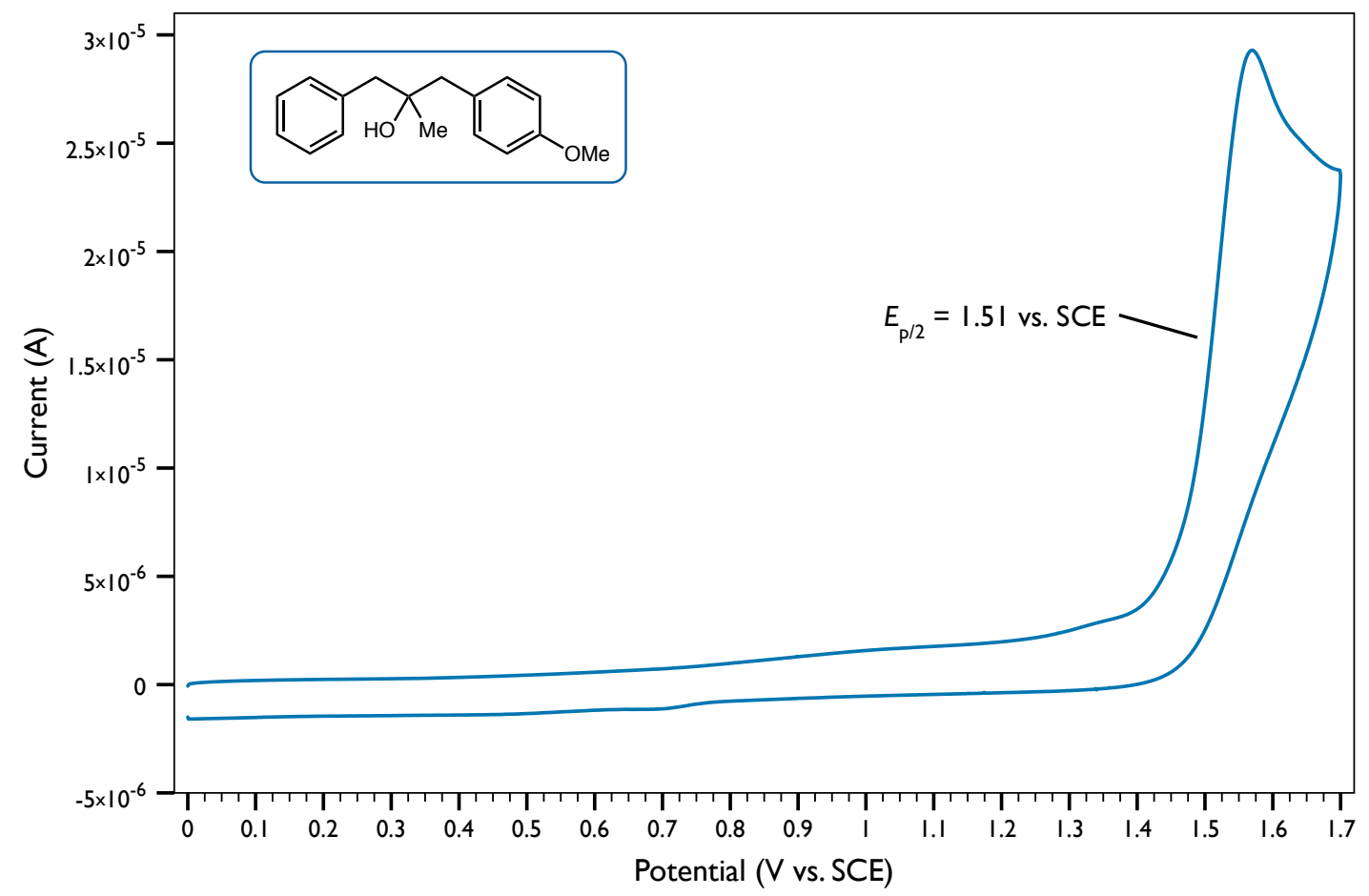

Figure S15. CV of 1-(4-methoxyphenyl)-2-methyl-3-phenylpropan-2-ol in $\mathrm{MeCN}$ 


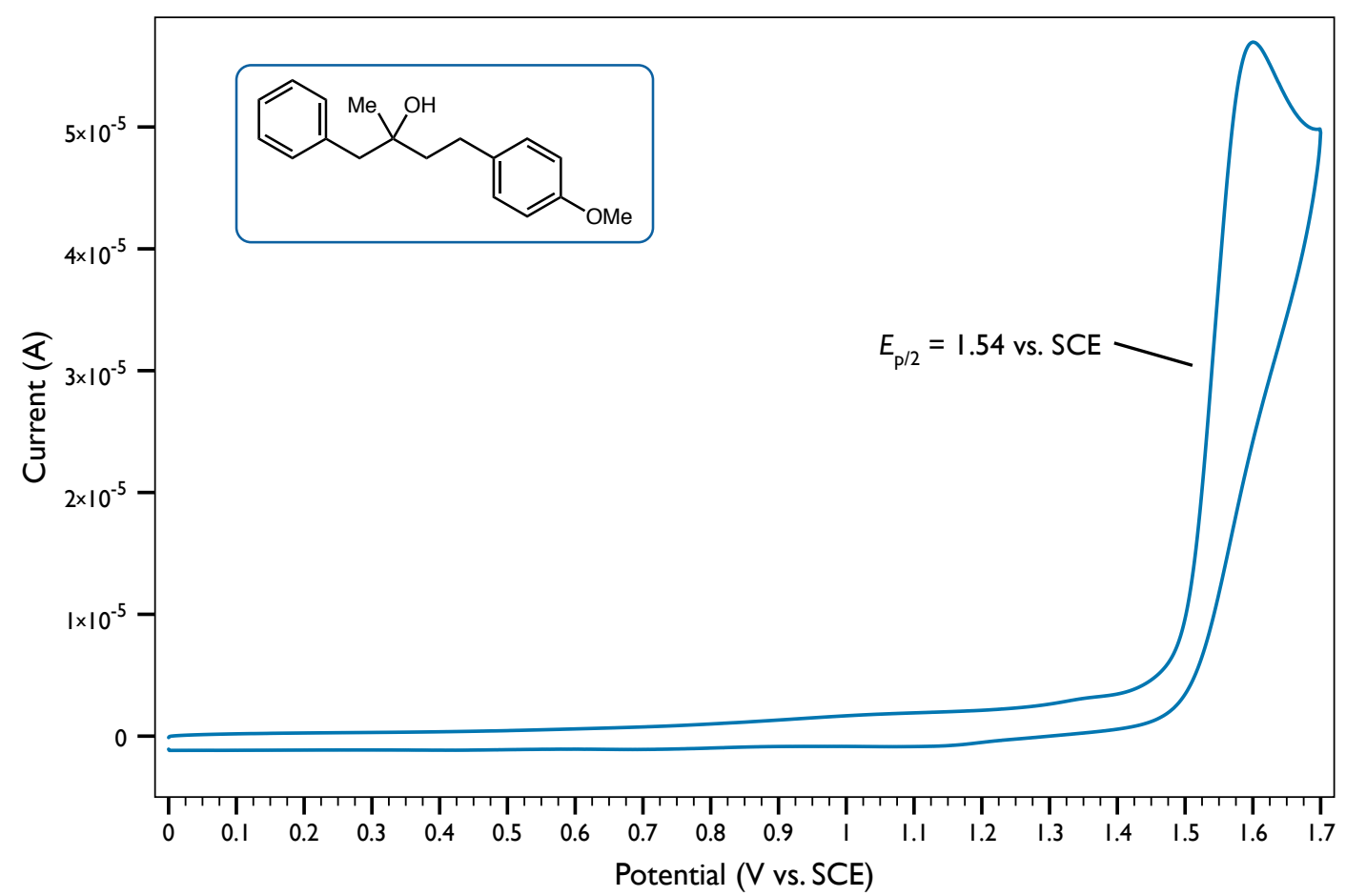

Figure S16. CV of 4-(4-methoxyphenyl)-2-methyl-1-phenylbutan-2-ol in $\mathrm{MeCN}$

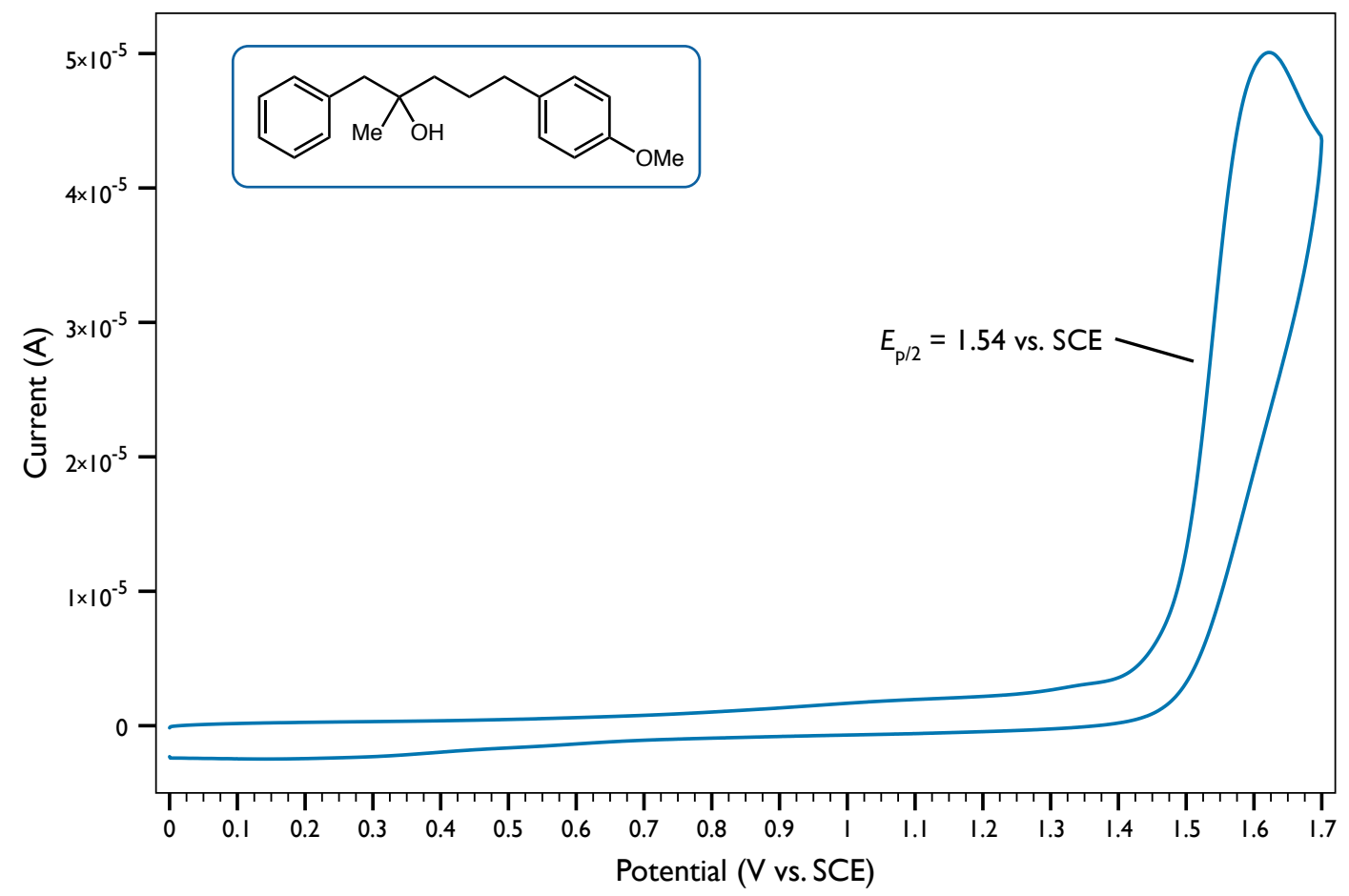

Figure S17. CV of 5-(4-methoxyphenyl)-2-methyl-1-phenylpentan-2-ol in $\mathrm{MeCN}$ 


\section{Structure and Potential Data of Photocatalysts}

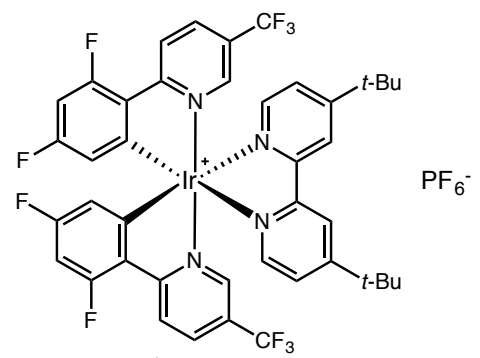

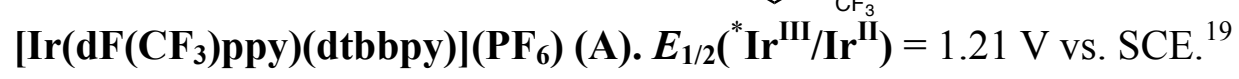

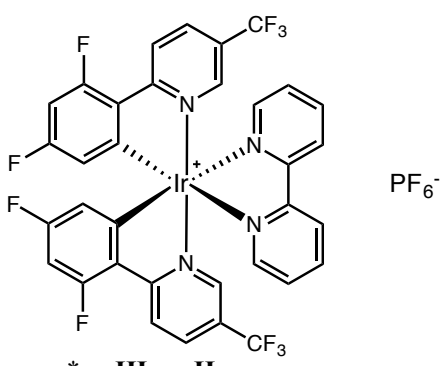

$\left[\operatorname{Ir}\left(\mathbf{d F}\left(\mathbf{C F}_{\mathbf{3}}\right) \mathbf{p p y}\right)(\mathbf{b p y})\right]\left(\mathbf{P F}_{\mathbf{6}}\right)(\mathbf{B}) . \boldsymbol{E}_{\mathbf{1 / 2}}\left(^{*} \mathbf{I r}^{\mathrm{III}} / \mathbf{I r}^{\mathrm{II}}\right)=1.32 \mathrm{~V} \mathrm{vs} . \mathrm{SCE}^{20}$

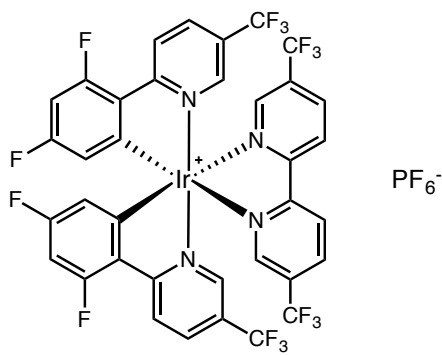

\section{$\left[\operatorname{Ir}\left(\mathrm{dF}\left(\mathrm{CF}_{3}\right) \mathrm{ppy}\right)\left(\mathbf{5 , 5} \mathrm{d}\left(\mathrm{CF}_{3}\right) \mathrm{bpy}\right)\right]\left(\mathrm{PF}_{6}\right)(\mathrm{C})$}

Ground state potentials were measured by $\mathrm{CV}$ in similar fashion to those in the previous section, at a $0.05 \mathrm{~V} / \mathrm{s}$ scan rate with a negative initial direction. Excited state reduction potential was calculated using the Rehm-Weller equation: ${ }^{21}$

$$
E_{\text {red }}^{\circ *}=E_{\text {red }}^{\circ}+E^{0-0}
$$

where $E^{\circ *}$ denotes the excited state reduction potential, $E^{\circ}$ the ground state reduction potential, and $E^{0-0}$ the energy difference between the $0^{\text {th }}$ vibration level of the ground state and that of the excited state. Due to the poor overlap between the absorption and emission spectra, $E^{0-0}$ is approximated as the high-energy onset of phosphorescence where the emission intensity is $10 \%$ of the obtained at the maximum emission wavelength, using the " $10 \%$ rule." 22 These estimations were corroborated by approximating the HOMO-LUMO gap as the difference between the onset of oxidation and the onset of reduction. ${ }^{23} \boldsymbol{E}_{\mathbf{1} / \mathbf{2}}\left(\mathbf{I r}^{\mathrm{III}} / \mathbf{I r}^{\mathrm{II}}\right)=-0.69 \mathrm{~V}$ vs. SCE; $\boldsymbol{E}_{\mathbf{1} / \mathbf{2}}\left({ }^{*} \mathbf{I r}^{\mathrm{III}} / \mathbf{I r} \mathbf{r}^{\mathrm{II}}\right)=1.68$ V vs. SCE. 

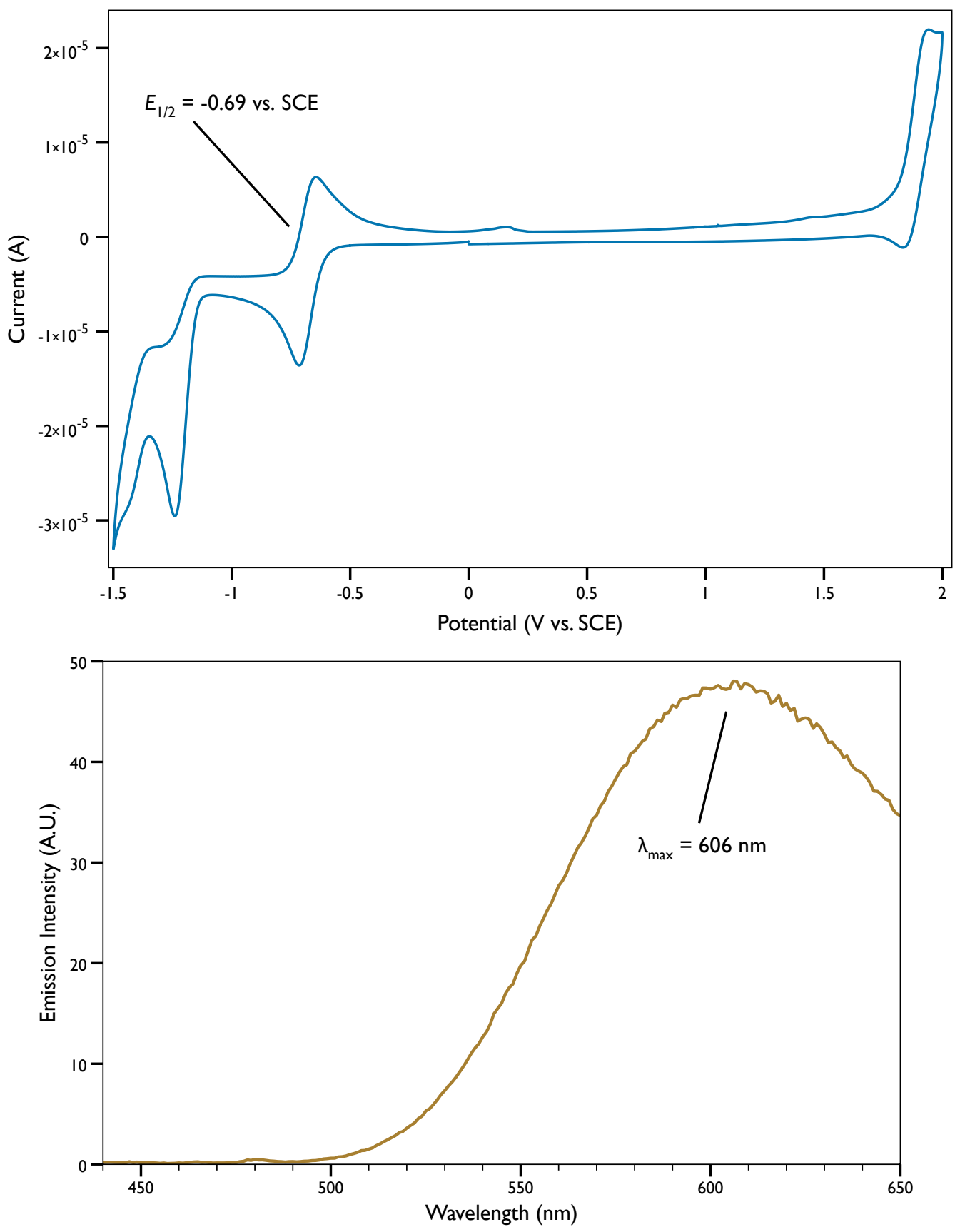

Figure S18. CV and phosphorescence spectrum of photocatalyst $\mathrm{C}$ in $\mathrm{MeCN}$. The emission spectrum was taken with excitation wavelength of $420 \mathrm{~nm}$. The emission maxima is at $606 \mathrm{~nm}$, thus the $10 \%$ of the maxima is at $524 \mathrm{~nm}$. 


\section{DFT Computations}

All calculations used DFT methodology ${ }^{24}$ as implemented in the Gaussian 09 series of computer programs. ${ }^{25}$ We employed the unrestricted B3LYP functional ${ }^{26}$ and the all-electron, valence triple- $\zeta$ plus polarization and diffuse function $6-311++\mathrm{G}(2 \mathrm{df}, 2 \mathrm{p})$ basis sets. ${ }^{27}$ Calculations were performed in the gas phase as well as with the CPCM polarizable conductor calculation model for acetonitrile. ${ }^{28}$ All molecules underwent geometry optimization, and stationary points were subjected to normal mode analysis.

\section{Calculation of $\mathrm{O}-\mathrm{H}$ bond energies:}

The bond energy was determined by calculating the energies of alcohol 4, its corresponding alkoxy radical and an $\mathrm{H}$-atom (Figure $\mathrm{S} 19 \mathrm{a}$ ). The $\mathrm{O}-\mathrm{H}$ bond energy of the alcohol starting material was referenced to the $\mathrm{O}-\mathrm{H} \mathrm{BDE}$ of $t-\mathrm{BuOH}(106.3 \mathrm{kcal} / \mathrm{mol})$ (Figure S19b). ${ }^{29}$

a<smiles>COc1ccc(C2(OP)CCCC2)cc1</smiles>
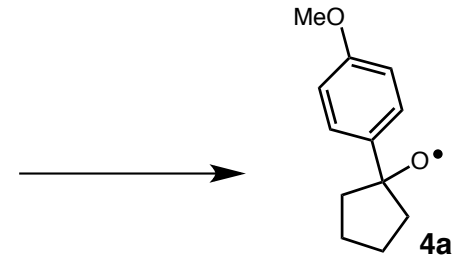

$4 a$

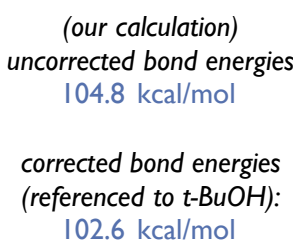

$102.6 \mathrm{kcal} / \mathrm{mol}$

(our calculation)

uncorrected bond energies<smiles>CC(C)(C)[O-]</smiles>

$H \bullet$

$106.3 \mathrm{kcal} / \mathrm{mol}$

Figure S19. Chemical equations for themodynamic analysis

Table S3. Thermodynamic parameters: Geometries and frequencies were calculated with UB3LYP/6-311++G(2df,2p) with addition of the CPCM (solvent = acetonitrile) solvation model. Energies are given in hartrees particle ${ }^{-1}$.

$\begin{array}{cccccc}\text { Entry } & \text { Job Name } & \text { E } & \text { G } & \text { H } & \text { S } \\ \text { I } & \text { tBuOH } & -233.77 \mid 05 I & -233.665 \mid 4 I & -233.62844 & 77.25 I \\ 2 & \text { tBuO-radical } & -233.0959008 & -233.003888 & -232.9668 I & 78.03 \\ 3 & \text { H-atom } & -0.502283065 & -0.512937 & -0.499923 & 27.392 \\ 4 & \mathbf{4} & -617.5620333 & -617.345275 & -617.29049 & 115.298 \\ 5 & \mathbf{4 a} & -616.8927875 & -616.689505 & -616.63494 & 114.853\end{array}$




\section{Calculation of $\mathbf{C}-\mathrm{C}$ bond energies:}

The bond strengths of the scissile $\mathrm{C}-\mathrm{C}$ bonds in 2-(4-methoxyphenyl)propan-2-ol (Figure S20a) and its corresponding alkoxy radical (Figure S20b) were estimated using DFT. To avoid the impact of ring strain on this BDE, 2-(4-methoxyphenyl)propan-2-ol was chosen as a model substrate to calculate the $\mathrm{C}-\mathrm{C}$ bond energy. Furthermore, this substrate allowed us to reference all bond energies to experimental ethane C-C bond energies (Figure S20c). ${ }^{30}$<smiles>COc1ccc(C([N+])(O)[N+]#N)cc1</smiles>

b<smiles>COc1ccc(C(C)(C)O)cc1</smiles>

c
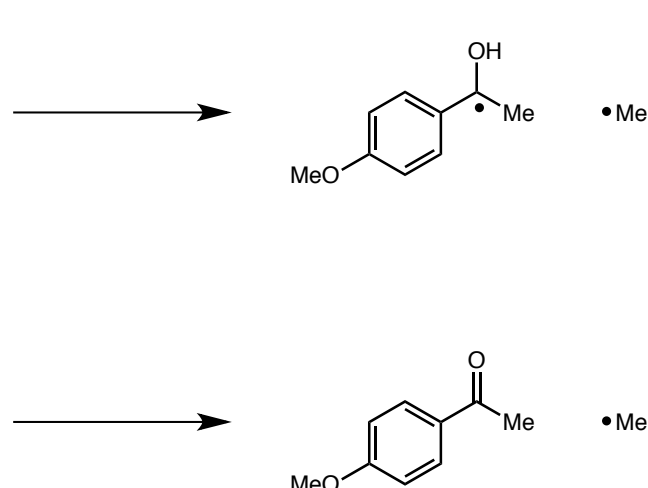

(our calculation) uncorrected $\mathrm{BDE}$ $-6.7 \mathrm{kcal} / \mathrm{mol}$ corrected $\mathrm{BDE}$
(referenced to $t-\mathrm{BuOH}$ ):
$-0.3 \mathrm{kcal} / \mathrm{mol}$

(our calculation) uncorrected $B D E$ $83.7 \mathrm{kcal} / \mathrm{mol}$

experimental $B D E$ : ref 30

$90.2 \mathrm{kcal} / \mathrm{mol}$

Figure S20. Chemical equations for themodynamic analysis

Table S4. Thermodynamic parameters: Geometries and frequencies were calculated with UB3LYP/6-311++G(2df,2p) with addition of the CPCM (solvent = acetonitrile) solvation model. Energies are given in hartrees particle ${ }^{-1}$.

$\begin{array}{cccccc}\text { Entry } & \text { Job Name } & \text { E } & \text { G } & \text { H } & \text { S } \\ \text { I } & \text { 2-(4-methoxyphenyl)propan-2-ol } & -540.1276347 & -539.945126 & -539.89384 & 107.94 \\ 2 & \text { 2-(4-methoxyphenyl)propan-2-ol ketyl } & -500.1625304 & -500.02159 \mid & -499.97093 & 106.63 \\ 3 & \text { 2-(4-methoxyphenyl)propan-2-oxy radical } & -539.4556213 & -539.286322 & -539.23474 & 108.57 \\ 4 & \text { I-(4-methoxyphenyl)ethan-I-one } & -2.954782878 & -499.468264 & -499.42058 & 100.35 \\ 5 & \text { Me radical } & -39.85846751 & -39.84694 \mid & -39.824865 & 46.462 \\ 6 & \text { Ethane } & -79.86192507 & -79.809124 & -79.783261 & 54.432\end{array}$




\section{Optimized geometries:}

Optimized geometries in Cartesian coordinates $(\AA)$ and energies (hartrees) for stationary points.

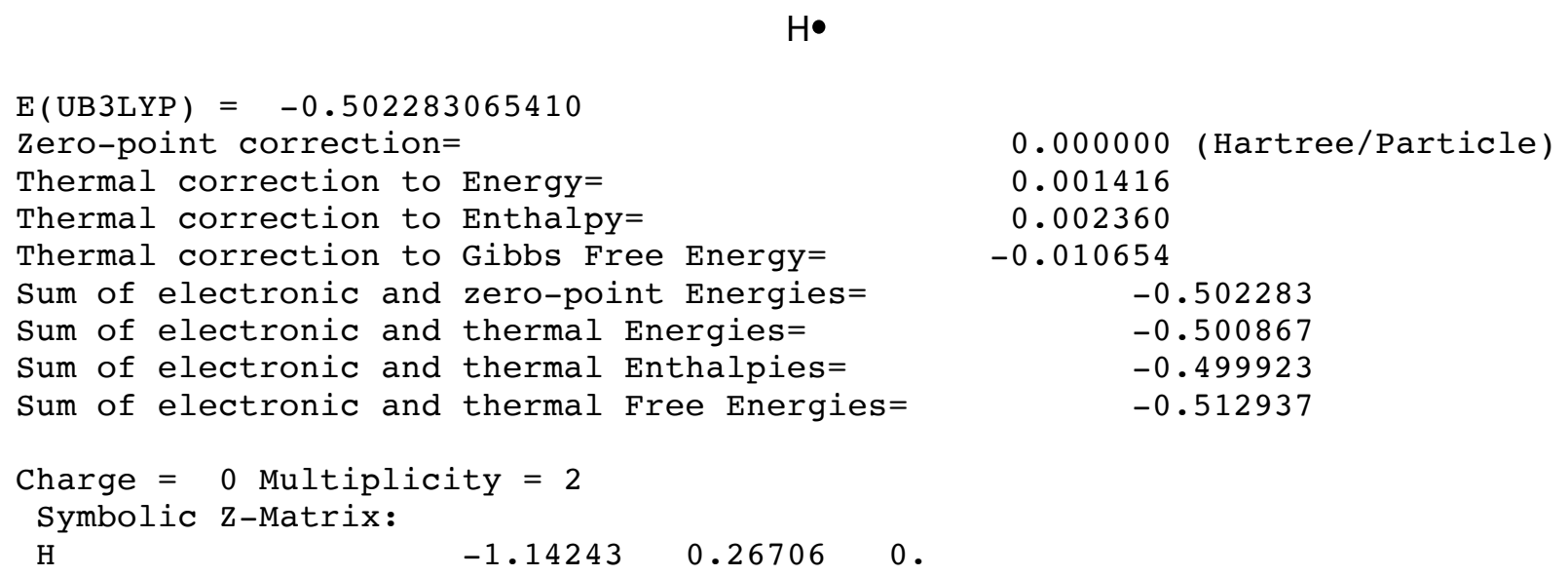




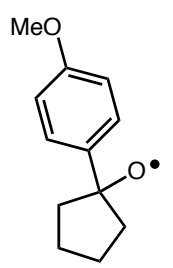

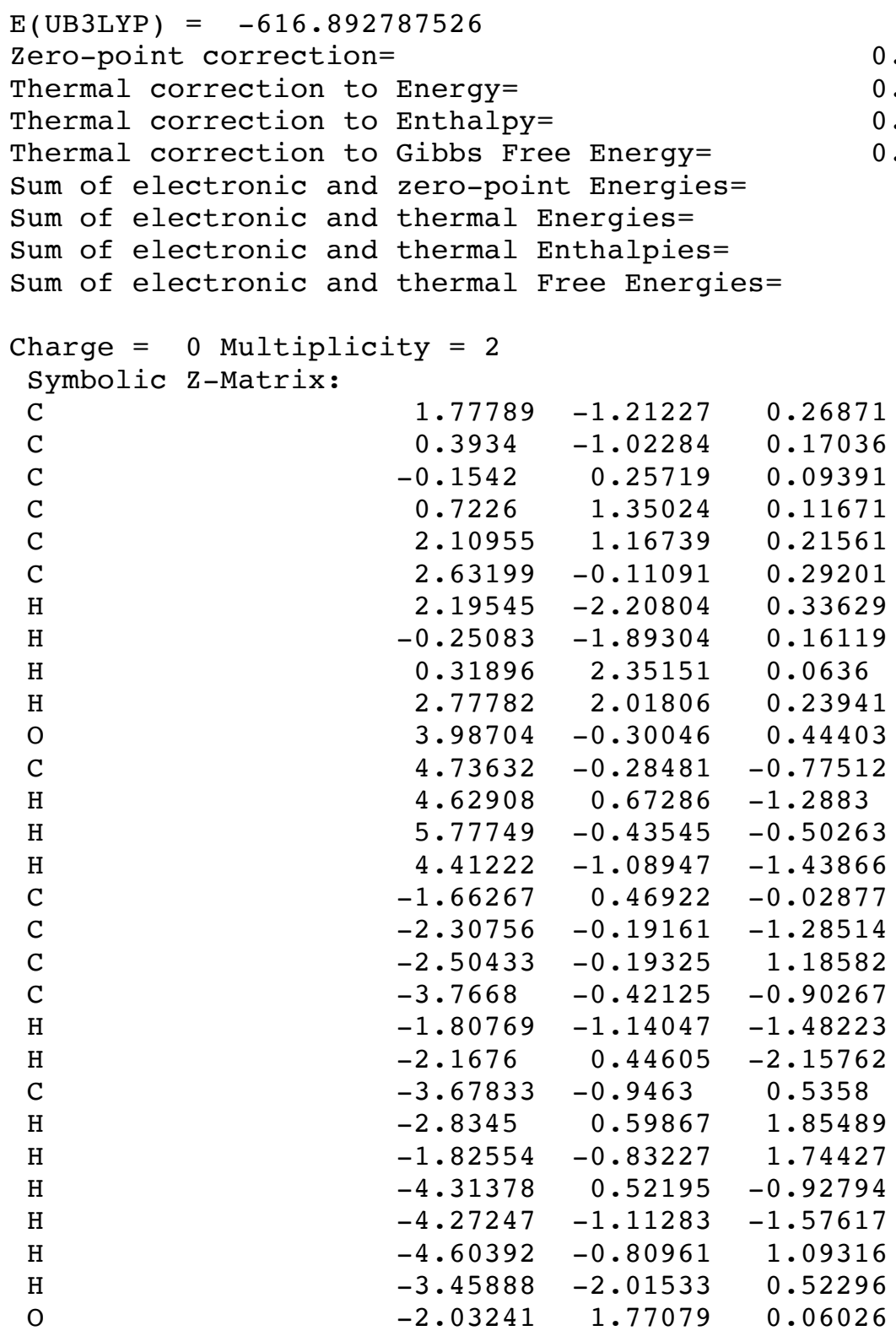




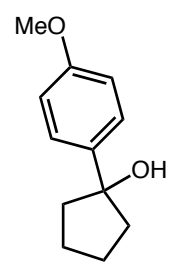

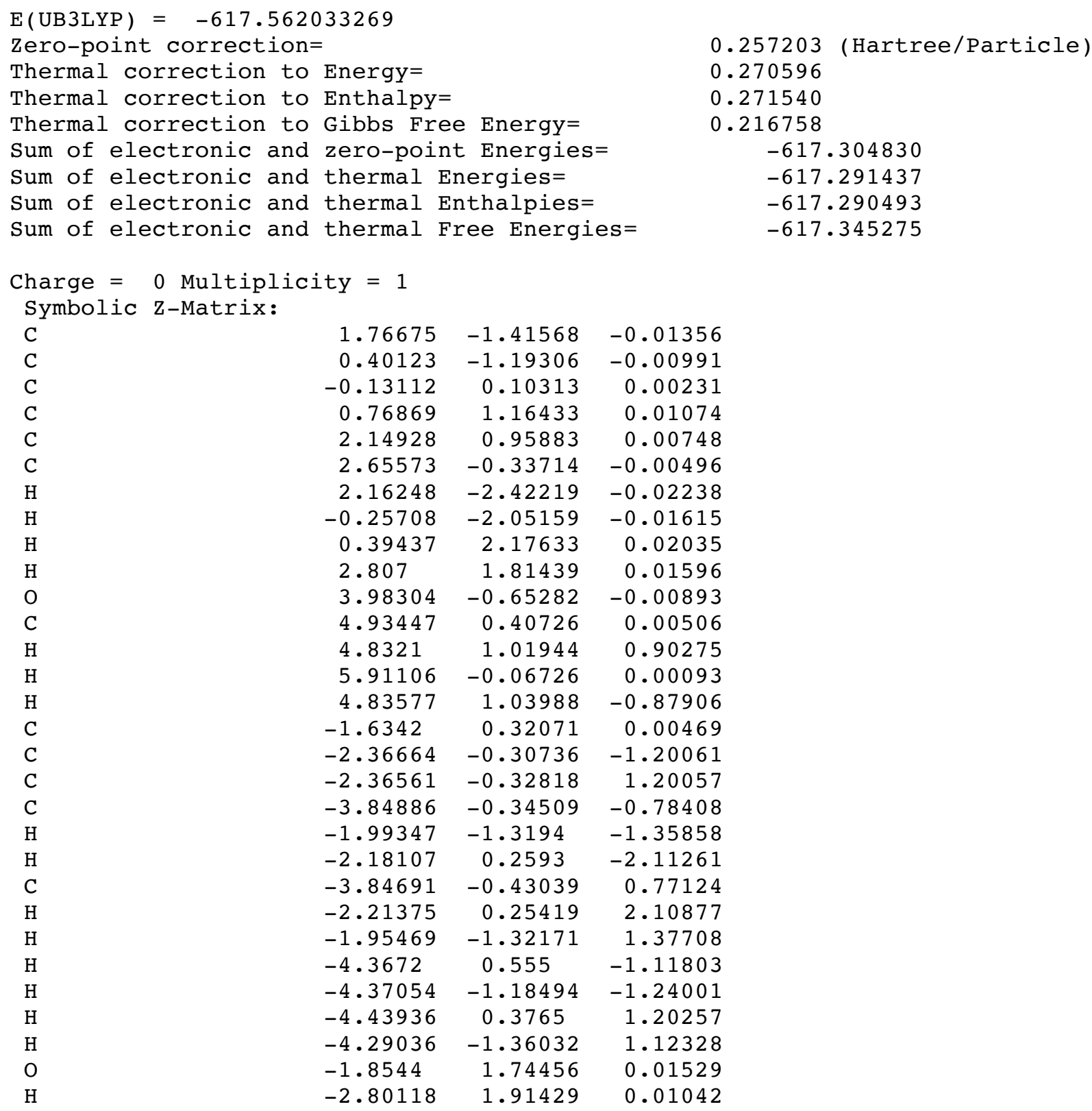




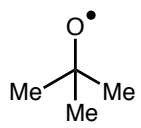

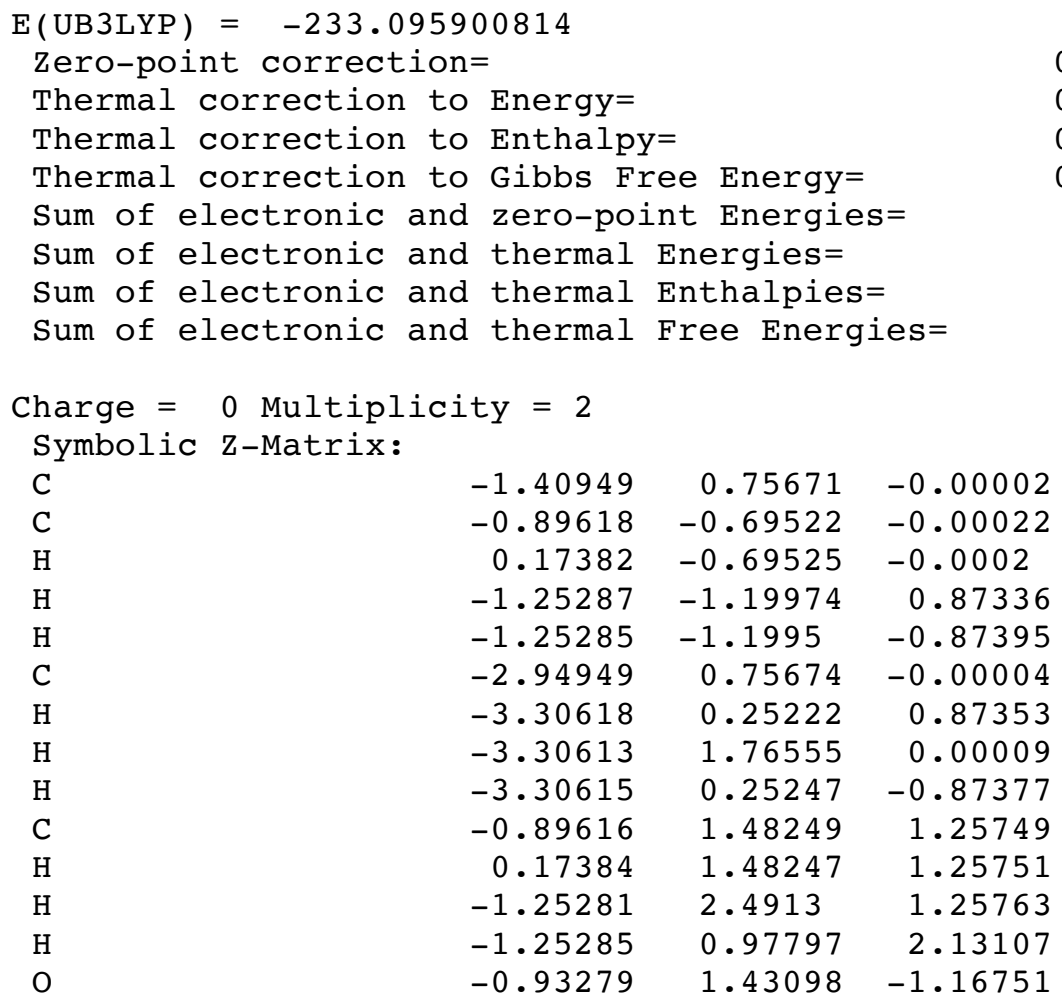




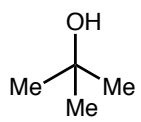

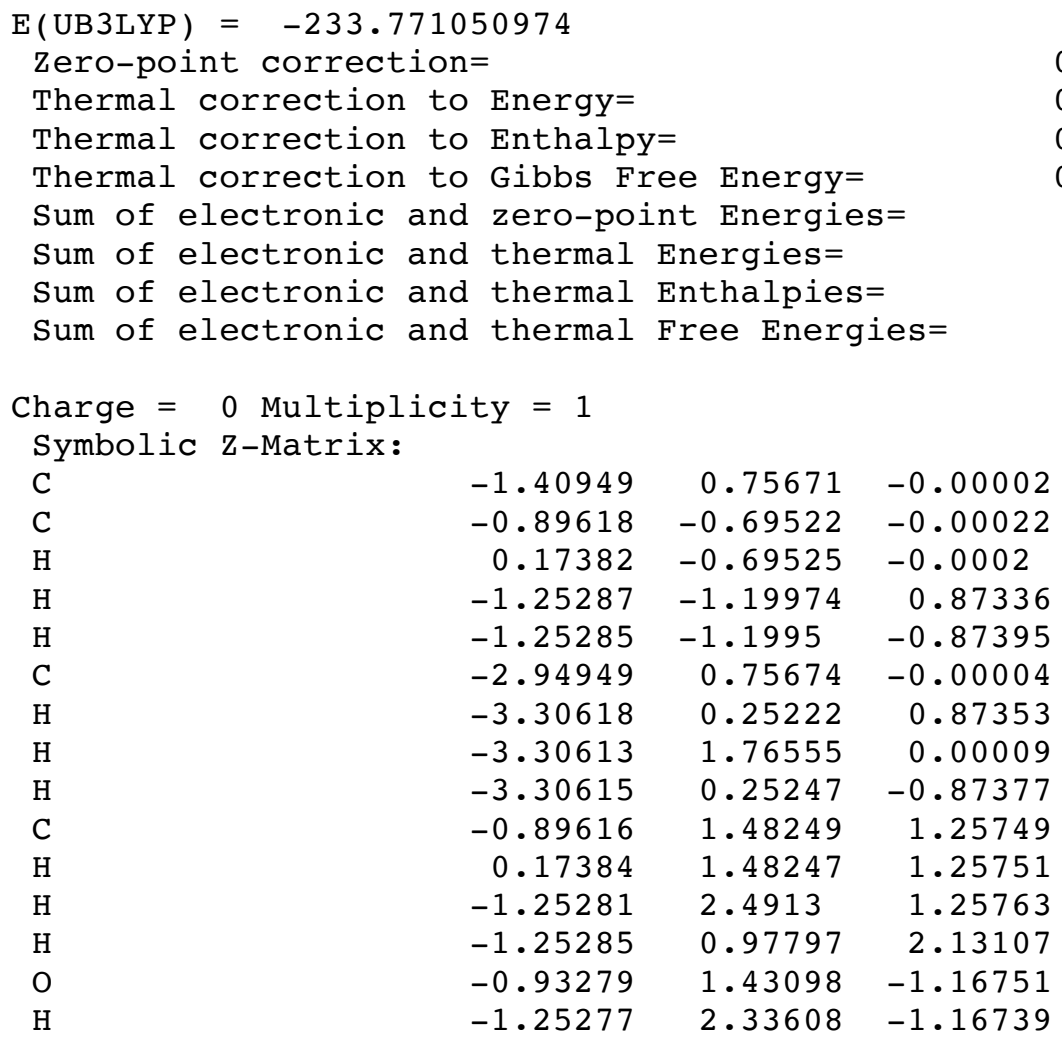




$$
\mathrm{H}_{3} \mathrm{C}-\mathrm{CH}_{3}
$$

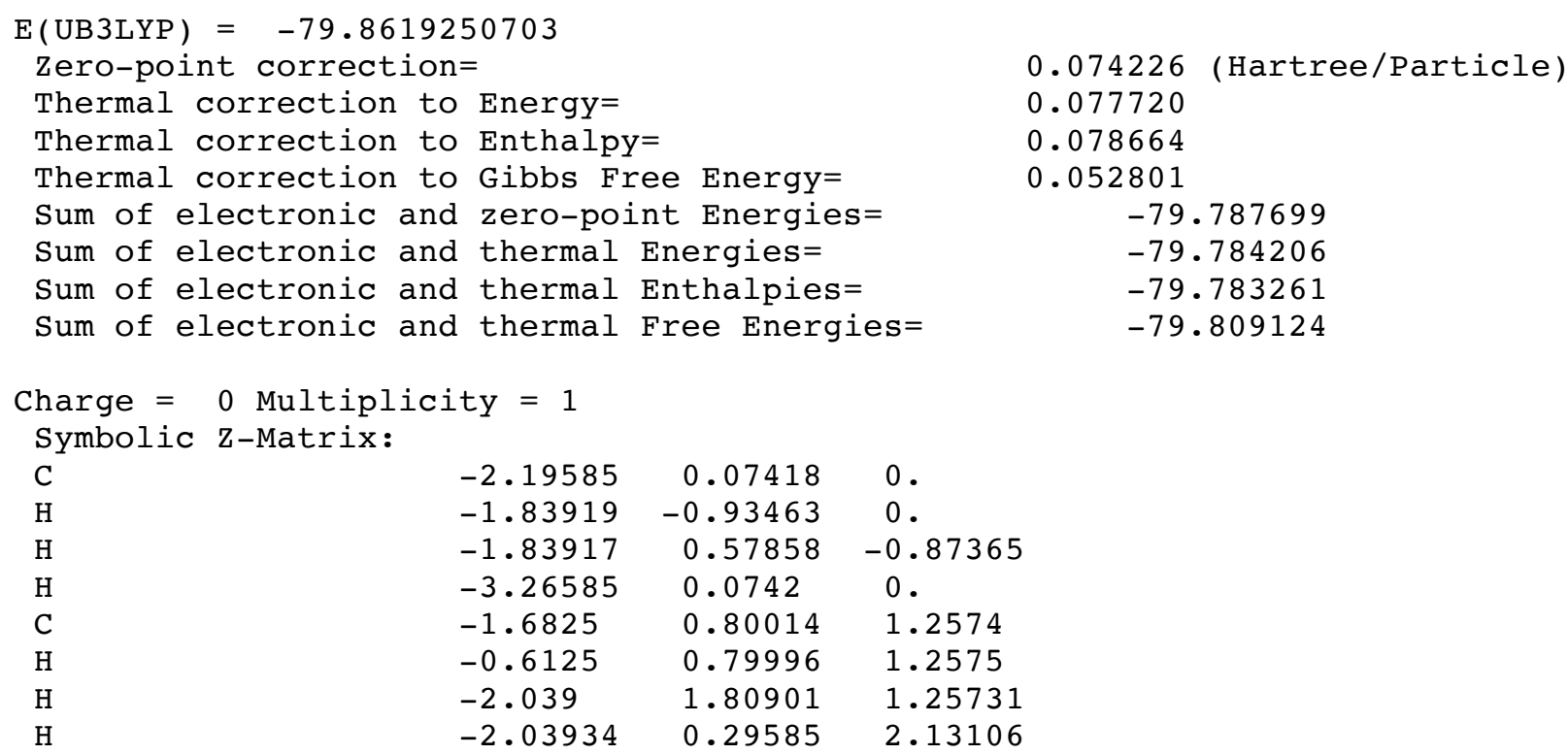


- $\mathrm{CH}_{3}$

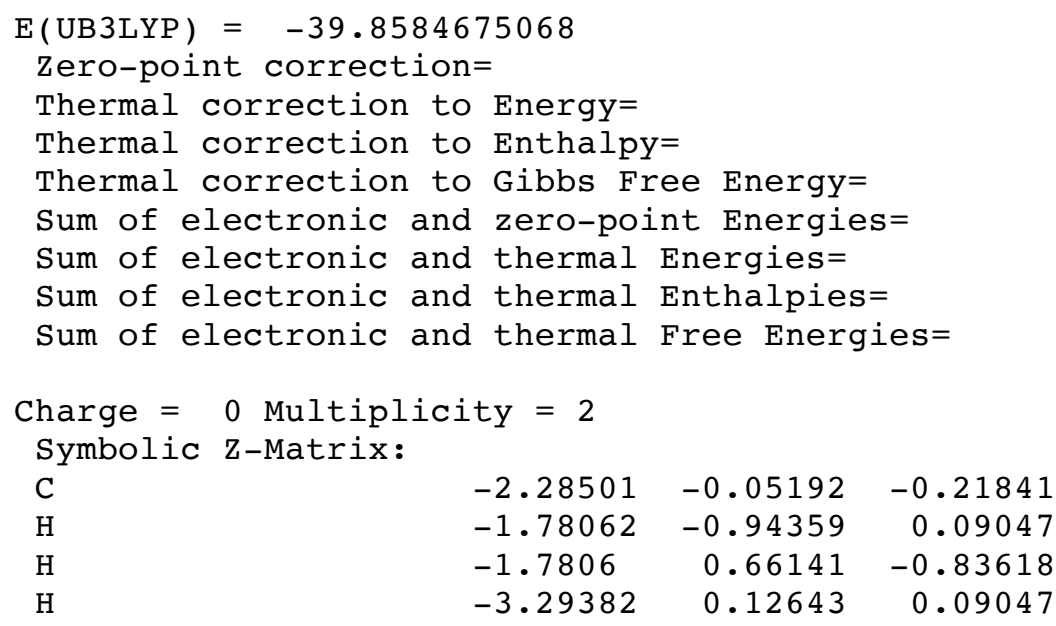




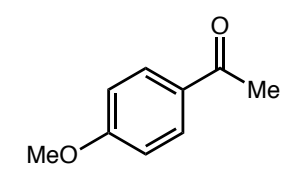

\begin{tabular}{|c|c|c|c|}
\hline \multicolumn{4}{|c|}{$E(U B 3 L Y P)=-499.601862578$} \\
\hline \multicolumn{4}{|c|}{$\begin{array}{l}\text { Zero-point correction= } \\
\text { Thermal correction to Energy= }\end{array}$} \\
\hline \multicolumn{4}{|c|}{ Thermal correction to Enthalpy= } \\
\hline \multicolumn{4}{|c|}{ Thermal correction to Gibbs Free Energy= } \\
\hline \multicolumn{4}{|c|}{ Sum of electronic and zero-point Energies= } \\
\hline \multicolumn{4}{|l|}{ Sum of electronic and } \\
\hline \multicolumn{4}{|l|}{ Sum of electronic and } \\
\hline Sum of electronic an & ad thermal & Free Enerc & ies $=$ \\
\hline \multicolumn{4}{|c|}{ Charge $=0$ Multiplicity $=1$} \\
\hline $\mathrm{C}$ & -0.36127 & 0.67459 & -0.52546 \\
\hline $\mathrm{C}$ & 1.00707 & 0.90273 & -0.72429 \\
\hline $\mathrm{C}$ & 1.59008 & 2.0972 & -0.28015 \\
\hline $\mathrm{C}$ & 0.80476 & 3.06353 & 0.36281 \\
\hline $\mathrm{C}$ & -0.56357 & 2.83539 & 0.56165 \\
\hline C & -1.14659 & 1.64092 & 0.11751 \\
\hline $\mathrm{H}$ & -0.80641 & -0.23741 & -0.86457 \\
\hline $\mathrm{H}$ & 1.60667 & 0.16492 & -1.21521 \\
\hline $\mathrm{H}$ & 1.2499 & 3.97554 & 0.70192 \\
\hline $\mathrm{H}$ & -1.16318 & 3.5732 & 1.05257 \\
\hline $\mathrm{C}$ & 3.09374 & 2.34791 & -0.49864 \\
\hline 0 & -2.54285 & 1.40812 & 0.32041 \\
\hline 0 & 3.61727 & 3.4205 & -0.09983 \\
\hline C & 3.95673 & 1.28601 & -1.2052 \\
\hline $\mathrm{H}$ & 4.95943 & 1.3375 & -0.83527 \\
\hline $\mathrm{H}$ & 3.55474 & 0.31343 & -1.01185 \\
\hline $\mathrm{H}$ & 3.95564 & 1.46929 & -2.25938 \\
\hline C & -2.73968 & 0.75689 & 1.5782 \\
\hline $\mathrm{H}$ & -3.78443 & 0.58269 & 1.73002 \\
\hline $\mathrm{H}$ & -2.21813 & -0.17739 & 1.58159 \\
\hline $\mathrm{H}$ & -2.36376 & 1.37807 & 2.36415 \\
\hline
\end{tabular}




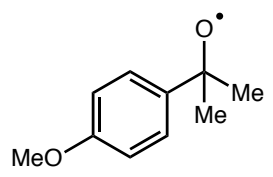

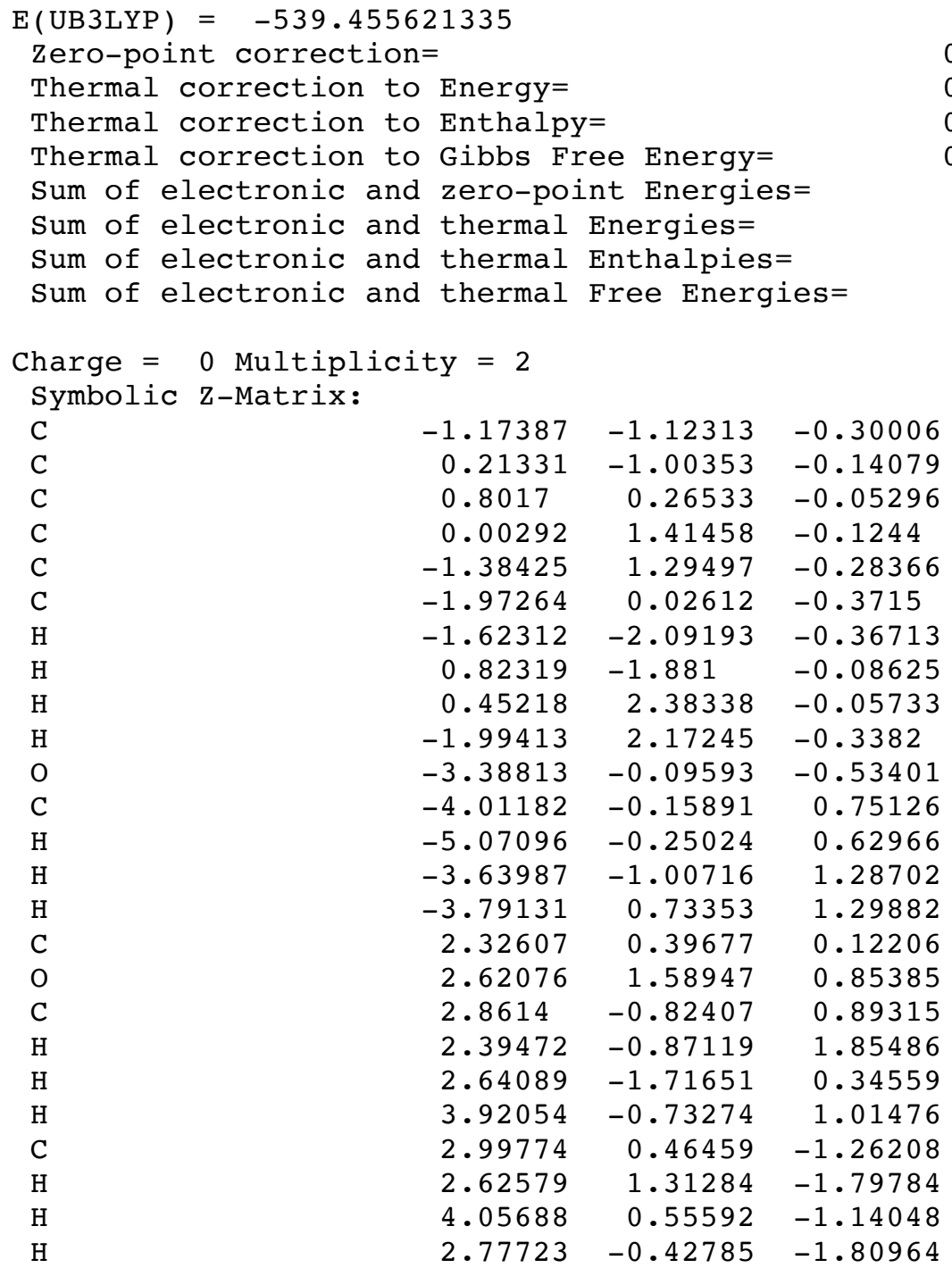




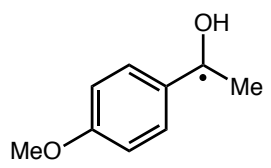

\begin{tabular}{|c|c|c|c|}
\hline \multicolumn{4}{|c|}{$E(U B 3 L Y P)=-500.162591296$} \\
\hline \\
\hline \multicolumn{4}{|c|}{ Thermal correction to Energy= } \\
\hline \multicolumn{4}{|c|}{ Thermal correction to Enthalpy= } \\
\hline \multicolumn{4}{|c|}{ Thermal correction to Gibbs Free Energy= } \\
\hline \\
\hline \multicolumn{2}{|c|}{$\begin{array}{l}\text { Sum of electronic and zero-point Energies= } \\
\text { Sum of electronic and thermal Energies= }\end{array}$} & Energies= & \\
\hline \multicolumn{4}{|l|}{ Sum of electronic and } \\
\hline \multicolumn{4}{|l|}{ Sum of electronic and } \\
\hline \multicolumn{4}{|c|}{$\begin{array}{cc}\text { Charge }= & 0 \text { Multiplicity }=2 \\
\text { Symbolic } & \text { z-Matrix: }\end{array}$} \\
\hline $\mathrm{C}$ & 1.14635 & 0.98634 & 0.00759 \\
\hline $\mathrm{C}$ & -0.23458 & 1.10612 & 0.00812 \\
\hline $\mathrm{C}$ & -1.08567 & -0.0285 & 0.01144 \\
\hline $\mathrm{C}$ & -0.44135 & -1.30333 & 0.00665 \\
\hline $\mathrm{C}$ & 0.93013 & -1.41671 & 0.00352 \\
\hline $\mathrm{C}$ & 1.74655 & -0.27604 & 0.00483 \\
\hline $\mathrm{H}$ & 1.74549 & 1.88464 & 0.01258 \\
\hline $\mathrm{H}$ & -0.65753 & 2.10033 & 0.00915 \\
\hline $\mathrm{H}$ & -1.04596 & -2.19764 & 0.00822 \\
\hline $\mathrm{H}$ & 1.40189 & -2.39053 & 0.0043 \\
\hline 0 & 3.09632 & -0.49664 & 0.01766 \\
\hline $\mathrm{C}$ & 3.96503 & 0.631 & -0.02567 \\
\hline $\mathrm{H}$ & 4.97612 & 0.23523 & -0.00963 \\
\hline $\mathrm{H}$ & 3.81604 & 1.21138 & -0.93879 \\
\hline $\mathrm{H}$ & 3.81853 & 1.27892 & 0.8409 \\
\hline C & -2.49081 & 0.07894 & 0.00725 \\
\hline 0 & -3.18979 & -1.10252 & -0.02588 \\
\hline C & -3.30029 & 1.32664 & -0.00099 \\
\hline $\mathrm{H}$ & -3.82301 & 1.45798 & -0.9551 \\
\hline $\mathrm{H}$ & -4.06671 & 1.2958 & 0.78004 \\
\hline $\mathrm{H}$ & -2.6946 & 2.21252 & 0.16966 \\
\hline $\mathrm{H}$ & -4.13459 & -0.92216 & 0.00807 \\
\hline
\end{tabular}




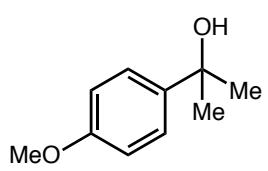

\begin{tabular}{|c|c|c|c|}
\hline \multicolumn{4}{|c|}{$E(U B 3 L Y P)=-540.127634724$} \\
\hline \multicolumn{4}{|c|}{ Thermal correction to Energy= } \\
\hline \multicolumn{4}{|c|}{ Thermal correction to Enthalpy= } \\
\hline \multicolumn{4}{|c|}{ Thermal correction to Gibbs Free Energy= } \\
\hline \multicolumn{4}{|l|}{ Sum of electronic and } \\
\hline \multicolumn{4}{|c|}{ Sum of electronic and thermal Energies= } \\
\hline \multirow{2}{*}{\multicolumn{4}{|c|}{ Sum of electronic and thermal Enthalpies= }} \\
\hline & nd thermal & Free Energ & ies $=$ \\
\hline \multicolumn{4}{|c|}{ Charge $=0$ Multiplicity $=1$} \\
\hline $\mathrm{C}$ & -1.17387 & -1.12313 & -0.30006 \\
\hline $\mathrm{C}$ & 0.21331 & -1.00353 & -0.14079 \\
\hline $\mathrm{C}$ & 0.8017 & 0.26533 & -0.05296 \\
\hline $\mathrm{C}$ & 0.00292 & 1.41458 & -0.1244 \\
\hline $\mathrm{C}$ & -1.38425 & 1.29497 & -0.28366 \\
\hline $\mathrm{C}$ & -1.97264 & 0.02612 & -0.3715 \\
\hline $\mathrm{H}$ & -1.62312 & -2.09193 & -0.36713 \\
\hline $\mathrm{H}$ & 0.82319 & -1.881 & -0.08625 \\
\hline $\mathrm{H}$ & 0.45218 & 2.38338 & -0.05733 \\
\hline $\mathrm{H}$ & -1.99413 & 2.17245 & -0.3382 \\
\hline 0 & -3.38813 & -0.09593 & -0.53401 \\
\hline $\mathrm{C}$ & -4.01182 & -0.15891 & 0.75126 \\
\hline $\mathrm{H}$ & -5.07096 & -0.25024 & 0.62966 \\
\hline $\mathrm{H}$ & -3.63987 & -1.00716 & 1.28702 \\
\hline $\mathrm{H}$ & -3.79131 & 0.73353 & 1.29882 \\
\hline C & 2.32607 & 0.39677 & 0.12206 \\
\hline 0 & 2.62076 & 1.58947 & 0.85385 \\
\hline $\mathrm{H}$ & 3.57102 & 1.67141 & 0.96295 \\
\hline C & 2.8614 & -0.82407 & 0.89315 \\
\hline $\mathrm{H}$ & 2.39472 & -0.87119 & 1.85486 \\
\hline $\mathrm{H}$ & 2.64089 & -1.71651 & 0.34559 \\
\hline $\mathrm{H}$ & 3.92054 & -0.73274 & 1.01476 \\
\hline $\mathrm{C}$ & 2.99774 & 0.46459 & -1.26208 \\
\hline $\mathrm{H}$ & 2.62579 & 1.31284 & -1.79784 \\
\hline $\mathrm{H}$ & 4.05688 & 0.55592 & -1.14048 \\
\hline $\mathrm{H}$ & 2.77723 & -0.42785 & -1.80964 \\
\hline
\end{tabular}




\section{References}

${ }^{1}$ Perrin, D. D.; Armarego, W. L. F. Purification of Laboratory Chemicals. $4^{\text {th }}$ ed.; ButterworthHeinemann: Oxford, 1997.

${ }^{2}$ Pangborn, A. B.; Giardello, M. A.; Grubbs, R. H.; Rosen, R. K.; Timmers, F. J. Organometallics, 1996, 15, 1518-1520.

${ }^{3}$ Rono, L. J.; Yayla, H. G.; Wang, D. Y.; Armstrong, M. F.; Knowles, R. R. J. Am. Chem. Soc., 2013, 135, 17735-17738.

${ }^{4}$ Canonne, P.; Bernatchez, M. J. Org. Chem. 1987, 52, 4025-4031.

${ }^{5}$ Paramahamsan, H.; Pearson, A. J.; Pinkerton, A. A.; Zhurova, E. A. Organometallics, 2008, 27, 900-907.

${ }^{6}$ Baraldi, P. G.; Barco, A.; Benetti, S.; Pollini, G. P.; Polo, E.; Simoni, D. J. Org. Chem. 1985, 50, 23-29.

${ }^{7}$ Cheon, C. H.; Kanno, O.; Toste, F. D. J. Am. Chem. Soc. 2011, 133, 13248-13251.

${ }^{8}$ Baciocchi, E.; Bietti, M.; Manduchi, L.; Steenken, S. J. Am. Chem. Soc. 1999, 121, 6624-6629.

${ }^{9}$ In a few cases, the reaction was run on $0.5 \mathrm{mmol}$ scale. Where it is the case, it will always be noted.

${ }^{10}$ Du, B.; Yuan, C.; Zhang, L.; Yang, L.; Liu, B. Tetrahedron Lett. 2013, 54, 2217-2220.

${ }^{11}$ Murata, R.; Hirano, K.; Uchiyama, M. Chem. Asian J. 2015, 10, 1286-1290.

12 Iranpoor, N.; Firouzabadi, H.; Khalili, D.; Motevalli, S. J. Org. Chem. 2008, 73, 4882-4887.

${ }^{13}$ Wang, X.; Liu, M.; Xu, L.; Wang, Q.; Chen, J.; Ding, J.; Wu, H. J. Org. Chem. 2013, 78, 5273-5281.

${ }^{14}$ Morandi, B.; Wickens, Z. K.; Grubbs, R. H. Angew. Chem. Int. Ed. 2013, 52, 2944-2948.

${ }^{15}$ Morandi, B.; Wickens, Z. K.; Grubbs, R. H. Angew. Chem. Int. Ed. 2013, 52, 2944-2948.

${ }^{16}$ Hayashi, M.; Shibuya, M.; Iwabuchi, Y. J. Org. Chem. 2012, 77, 3005-3009.

${ }^{17}$ Yasu, Y.; Koike, T.; Akita, M. Adv. Synth. Catal. 2012, 354, 3414-3420.

${ }^{18}$ Houmam, A. Chem. Rev. 2008, 108, 2180-2237.

${ }^{19}$ Shaw, M. H.; Shurtleff, V. W.; Terret, J. A.; Cuthbertson, J. D.; MacMillan, D. W. C. Science, 2016, 352, 1304-1308.

${ }^{20}$ Hanss, D.; Freys, J. C.; Bernardinelli, G.; Wenger, O. S. Eur. J. Inorg. Chem. 2009, 48504859.

${ }^{21}$ Brennan, J. L.; Keyes, T. E.; Forster, R. J. Langmuir 2006, 22, 10754-10761.

22 (a) Dossing, A.; Ryu, C. K.; Kudo, S.; Ford, P. C. J. Am. Chem. Soc. 1993, 115, 5132-5137. (b) Bruner, B.; Walker, M. B.; Ghimire, M. M.; Zhang, D.; Selke, M.; Klausmeyer, K. K.; Omary, M. A.; Farmer, P. J. Dalton Trans. 2014, 43, 11548-11556. (c) McClure, L. J.; Ford, P. C. J. Phys. Chem. 1992, 96, 6640-6650. (d) Schlenker, C. W.; Thompson, M. E. Top. Curr. Chem. 2012, 312, 175-212.

${ }^{23}$ (a) Ye, C.; Li, M.; Luo, J.; Chen, L.; Tang, Z.; Pei, J.; Jiang, L.; Song, Y.; Zhu. D. J. Mater. Chem. 2012, 22, 4299-4305. (b) Singh, A.; Teegardin, K.; Kelly, M.; Prasad, K. S.; Krishnan, S.; Weaver, J. D. J. Organomet. Chem. 2015, 776, 51-59.

${ }^{24}$ Parr, R. G.; Yang, W. Density-Functional Theory of Atoms and Molecules. University Press: Oxford, 1989. 
${ }^{25}$ Gaussian 09, Revision C.01, Frisch, M. J.; Trucks, G. W.; Schlegel, H. B.; Scuseria, G. E.; Robb, M. A.; Cheeseman, J. R.; Scalmani, G.; Barone, V.; Mennucci, B.; Petersson, G. A.; Nakatsuji, H.; Caricato, M.; Li, X.; Hratchian, H. P.; Izmaylov, A. F.; Bloino, J.; Zheng, G.; Sonnenberg, J. L.; Hada, M.; Ehara, M.; Toyota, K.; Fukuda, R.; Hasegawa, J.; Ishida, M.; Nakajima, T.; Honda, Y.; Kitao, O.; Nakai, H.; Vreven, T.; Montgomery, J. A., Jr.; Peralta, J. E.; Ogliaro, F.; Bearpark, M.; Heyd, J. J.; Brothers, E.; Kudin, K. N.; Staroverov, V. N.; Kobayashi, R.; Normand, J.; Raghavachari, K.; Rendell, A.; Burant, J. C.; Iyengar, S. S.; Tomasi, J.; Cossi, M.; Rega, N.; Millam, J. M.; Klene, M.; Knox, J. E.; Cross, J. B.; Bakken, V.; Adamo, C.; Jaramillo, J.; Gomperts, R.; Stratmann, R. E.; Yazyev, O.; Austin, A. J.; Cammi, R.; Pomelli, C.; Ochterski, J. W.; Martin, R. L.; Morokuma, K.; Zakrzewski, V. G.; Voth, G. A.; Salvador, P.; Dannenberg, J. J.; Dapprich, S.; Daniels, A. D.; Farkas, Ö.; Foresman, J. B.; Ortiz, J. V.; Cioslowski, J.; Fox, D. J. Gaussian, Inc., Wallingford CT, 2010.

${ }^{26}$ (a) Parr, R. G.; Yang, W. Annu. Rev. Phys. Chem. 1995, 46, 701-728. (b) Stephens, P. J.; Devlin, F. J.; Chabalowski, C. F.; Frisch, M. J. J. Phys. Chem. 1994, 98, 11623-11627. (c) Lee, C.; Yang, W.; Parr, R. G. Phys. Rev. B 1988, 37, 785-789. (d) Becke, A. D. Phys. Rev. A 1988, 38, 3098-3100. (e) Becke, A. D. J. Chem. Phys. 1993, 98, 5648-5652.

${ }^{27}$ (a) McLean, A. D.; Chandler, G. S. J. Chem. Phys. 1980, 72, 5639-5648. (b) Raghavachari, K.; Binkley, J. S.; Seeger, R.; Pople, J. A. J. Chem. Phys. 1980, 72, 650-654. (c) Blaudeau, J.-P.; McGrath, M. P.; Curtiss, L. A., Radom, L. J. Chem. Phys. 1997, 107, 5016-5021. (d) Wachters, A. J. H. J. Chem. Phys. 1970, 52, 1033. (e) Hay, P. J. J. Chem. Phys. 1977, 66, 4377-4384. (f) Raghavachari, K.; Trucks, G. W. J. Chem. Phys. 1989, 91, 1062-1065. (g) Binning Jr., R. C.; Curtiss, L. A. J. Comput. Chem. 1990, 11, 1206-1216. (h) McGrath, M. P.; Radom, L. J. Chem. Phys. 1991, 94, 511-516. (i) Curtiss, L. A.; McGrath, M. P., Blaudeau, J.-P.; Davis, N. E.; Binning Jr., R. C.; Radom, L. J. Chem. Phys. 1995, 103, 6104-6113. (j) Clark, T.; Chandrasekhar, J.; Spitznagel, G. W.; Schleyer, P. v. R. J. Comput. Chem. 1983, 4, 294-301. (k) Frisch, M. J.; Pople, J. A.; Binkley, J. S. J. Chem. Phys. 1984, 80, 3265-3269.

28 (a) Barone, V.; Cossi, M. J. Phys. Chem. A., 1998, 102, 1995-2001. (b) Cossi, M.; Rega, N.; Scalmani, G.; Barone, V. J. Comput. Chem., 2003, 24, 669-681.

${ }^{29}$ Luo, Y.-R. Comprehensive Handbook of Chemical Bond Energies; CRC Press: Boca Raton, FL, 2007, p 258.

${ }^{30}$ Luo, Y.-R. Comprehensive Handbook of Chemical Bond Energies; CRC Press: Boca Raton, FL, 2007, p 147. 